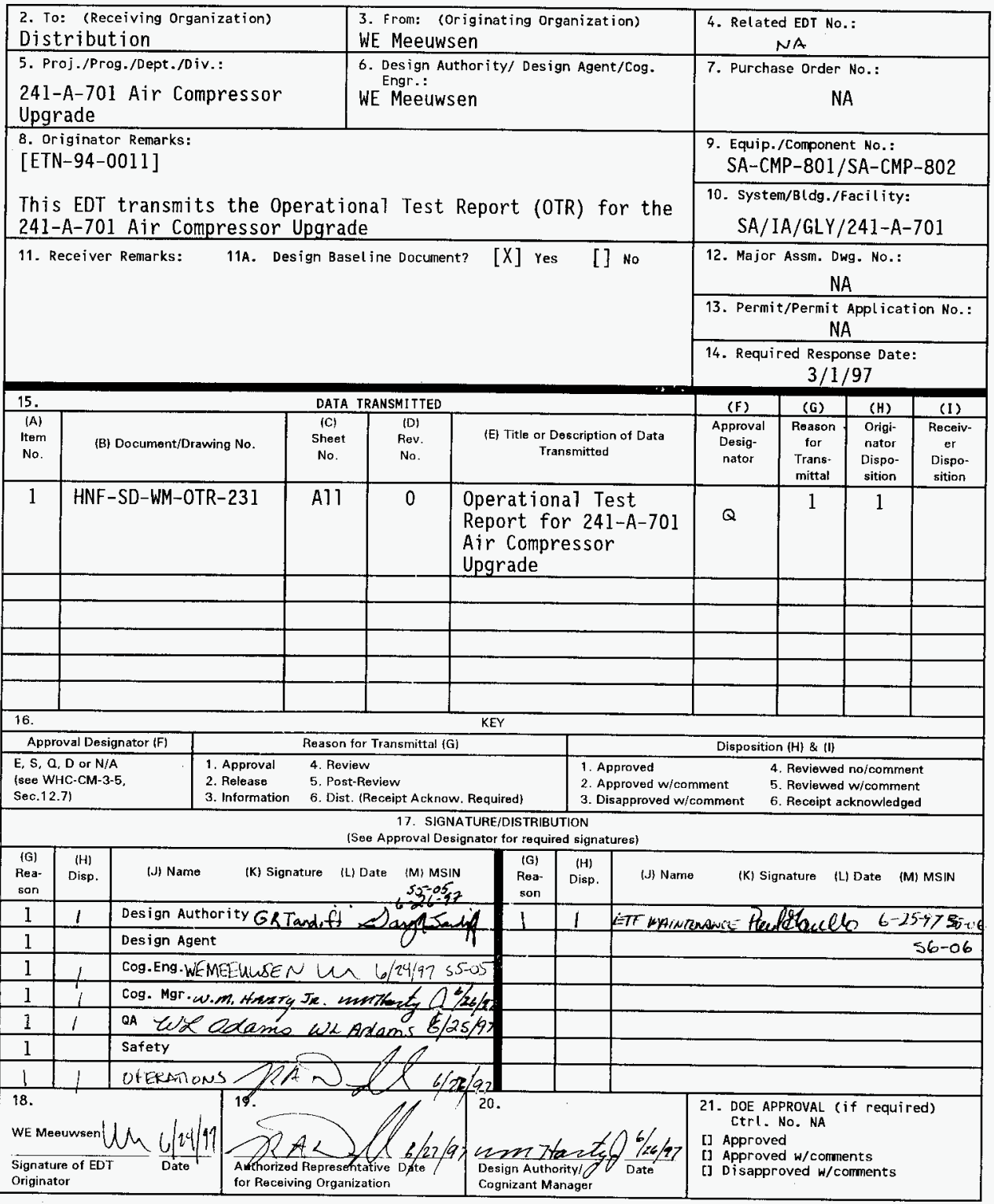




\section{OPERATIONAL TEST REPORT FOR THE 241-A-701 AIR COMPRESSOR UPGRADE}

\section{WE MEEUWSEN}

Lockheed Martin Hanford, Richland, WA 99352

U.S. Department of Energy Contract DE-AC06-96RL13200
EDT/ECN: $\quad 618381$
Org Code: 74130
UC: 2030
B\&R Code: EW3120071
Charge Code: N14A3
Total Pages: 170,71 KMB $6 / 50 / 97$

Key Words: 241-A-701, 701-A, Compressor, Air Compressor, Compressed Air System, Operational Test Report, OTR

Abstract: The attached Operational Test Report (OTR) documents the results of the Operational Test Procedure (0TP-200-003) for the two Ingersol1-Rand stationary air compressors installed per 2E-93-1324.

Ingersol1-Rand is a trademark of Ingersoll-Rand $\mathrm{CO}$.

Hankison is a trademark of Hankison Corp.

Sensatherm is a trademark of Hankison Corp.

Hydr-0-Thrift is a trademark of Hydro-0-Thrift Corp.

TRADEMARK DISCLAIMER. Reference herein to any specific commercial product, process, or service by trade name, trademark, manufacturer, or otherwise, does not necessarily constitute or imply its endorsement, recommendation, or favoring by the United states Government or any agency thereof or $i$ ts contractors or subcontractors.

Printed in the United States of America. To obtain copies of this document, contact: Document Control Services, P.O. Box 950, Mailstop H6-08, Richland WA 99352, Phone (509) 372-2420; Fax (509) 376-4989.
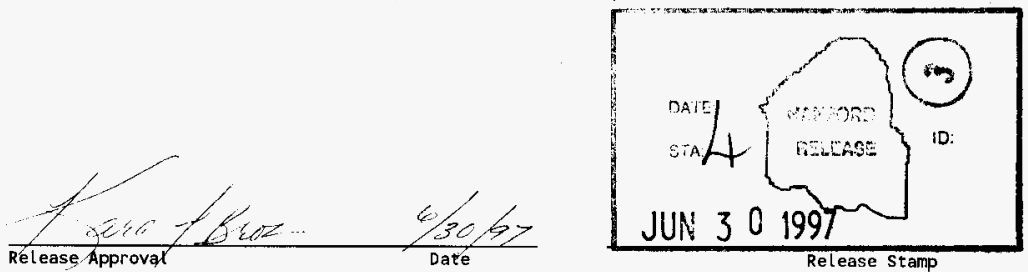

Approved for Public Release 


\section{OPERATIONAL TEST REPORT FOR 241-A-701 AIR COMPRESSOR UPGRADE}

MAY 12, 1997

WE MEEUWSEN

SECTION

PAGE

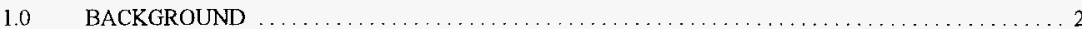

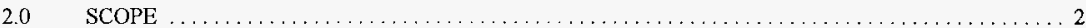

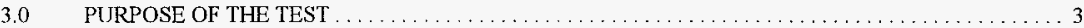

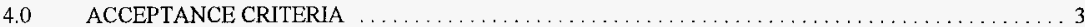

$4.1 \quad$ GRAPHS OF RECORDED TEST DATA FOR SA-CMP-801 $\ldots \ldots \ldots \ldots \ldots \ldots \ldots \ldots \ldots 6$

4.2 GRAPHS OF RECORDED TEST DATA FOR SA-CMP-802 $\ldots \ldots \ldots \ldots \ldots \ldots \ldots \ldots \ldots \ldots \ldots \ldots$

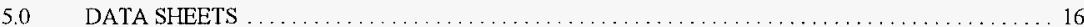

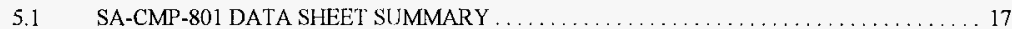

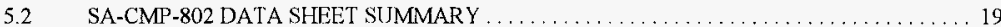

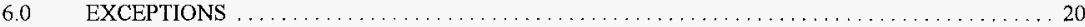

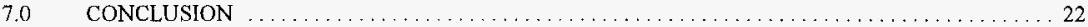

8.0 COMPLETED COPY OF OTP-200-003 PROCEDURE STEPS $\ldots \ldots \ldots \ldots \ldots \ldots \ldots \ldots \ldots \ldots \ldots$ 
An operability test for the compressed air system at 241 -A-701 was performed between $3 / 10 / 97$ and $3 / 19 / 97$. The test was executed using the Operability Test Procedure (OTP) OTP-200-003, revision 1. The purpose of this Operational Test Procedure was to provide instructions on verifying that 241-A-701 Air Compressors and associated components meet the functional, operational and design requirements with all items in the final inservice configuration. The air system was upgraded through work instructions provided in work package $2 \mathrm{E}-93$ 1324, "241-A-701 Compressed Air System Upgrade/Replacement". This Operational Test Report (OTR) documents the successful completion of the OTP' as well as providing a copy of the OTP that was completed during the performance of the operability test.

OTP-200-003 was used for testing the operability of 701-A compressor system and is a rewrite of the original test plan, WHC-SD-WM-OTP-169. The original test plan was replaced by OTP-200-003 because the original test plan was completed intermittently over several months; as a result several data sheets were missing, steps in the OTP were omitted or not signed off', and portions of the system were not tested satisfactorily. OTP-200-003 is based on the Operating Procedure TO-200-610, "Operate the 701-A Compressors" and the original OTP, WHCSD-WM-OTP-169.

\section{$\underline{\text { SCOPE }}$}

The scope of the OTP included operational testing of the following components in their final in service configuration:

- Two new 125 Horsepower (Hp) Ingersoll-Rand PHE-NL, two stage, horizontal reciprocating compressors. Each Ingersoll-Rand PHE-NL is rated to deliver $433 \mathrm{scfm}$ at 150 psig. These compressors will be cooled by a $50 / 50$ glycol and water mixture in a closed loop. Each compressor is equipped with an intercooler, aftercooler and moisture separators.

- Hydro-O-Thrift air cooled heat exchanger; designed to cool $67 \mathrm{gpm}$ of $40 \%$ glycol from $135^{\circ} \mathrm{F}$ to $115^{\circ} \mathrm{F}$ at an ambient dry bulb design temperature of $110^{\circ} \mathrm{F}$ (the heat exchanger is located outside of building 241-A-701). The interconnecting piping, pump skid and surge tank are located inside building 241-A-701.

- Hankison heatless DH-165 desiccant compressed air dryer, designed to receive 165 $\mathrm{scfm}$ at $100 \mathrm{psig}$ of compressed air, and deliver approximately $136 \mathrm{scfm}$ at $-40^{\circ} \mathrm{F}$ dew point. The dryer is equipped with a Hankison Sensatherm automatic purge saving system.

The aging waste ventilation system contains three fans; two are located in 702-A while the third is the $4000 \mathrm{cfm}$ portable exhauster. Each of these fans has its own filter train. The filter train associated with the operating fan is "open" or "valved in" by positioning a butterfly valve with a pneurnatic actuator. The other two non operating fans and associated filter trains are "closed" or "valved out" by positioning separate butterfly valves on each filter train. These butterfly valves are also pneumatically positioned. The air used to position these butterfly valves is received from the accumulator mentioned above. This accumulator is fed from the 701-A compressor system. The accumulator provides redundancy in itself by being sized sufficiently to cycle any given piston actuator 10 times as a result of fan switchover. Testing of the redundancy and the capability of fan switchover was determined to be beyond the scope and was therefore not tested under OTP-200-003. Testing of these systems will be performed at a later date. 
A description and safety class designation of the accumulator and 701-A compressor system is contained in WHCSD-WM-DA-137, Safety Classification of the 241-A-701 Compressed Air System and shown on drawings H-262895, Sheet 2 and H-14-20308, Sheet 3. The design bases for the 241-A-702 Ventilation System Accumulator is contained in WHC-SD-WM-DB-016, 241-A-702 Ventilation System Accumulator Design Basis.

\subsection{PURPOSE OF THE TEST}

The purpose of the OTP was to:

- Verify the system operates within its intended design parameters.

- Provide a final function check for the entire upgraded air system

- Demonstrate the daily operation of the system.

- Collect baseline data concerning system performance.

- Identify any additional items needed for safe system performance.

\section{ACCEPTANCE CRITERIA}

The instruments listed on page 9 of OTP-200-003 were used for meeting acceptance criteria during the testing of SA-CMP-801 and SA-CMP-802. Some of the readings taken from these instruments have been shown graphically in sections 4.1 and 4.2 of this OTR except for the following instruments:

GLY-FG-802, "SA-CMP-801 HP SIDE GLY FLO"

GLY-FG-806, "SA-CMP-802 HP SIDE GLY FLO"

IA-FI-801, "LA FLOW INDICATOR"

The first two instruments were not shown graphically since the instrument provides a qualitative, rather than quantitative, reading. The readings taken from these instruments is the confirmation that coolant is or isn't flowing. All data sheet entries for these two instruments show that flow was maintained throughout the entire operational test and therefore successfully meeting the acceptance criteria. The third instrument listed above is not shown for reasons given in exception 6 below.

The remaining instruments and their readings which were used for meeting the acceptance criteria during testing of SA-CMP-801 are listed on the following pages. 


\section{SA-PI-801 "REMOTE BRG OIL PRESS"}

The recorded readings for oil pressure on SA-CMP-801 ranged from a high of $48 \mathrm{psi}$ at the start of the test to a low of approximately $20 \mathrm{psi}$. All readings were well within the $13.5-71.5(15-65$ with $10 \%$ margin) psi acceptance criteria range stated on page 9 of the operational test procedure and is therefore acceptable. This data is shown graphically in section 4.1 and copies of the actual data sheets generated during the test are contained in section 8 of this test report.

\section{SA-PISL-801 "SA HDR LO PRESS ALARM"}

The recorded readings for header pressure during testing of SA-CMP-801 ranged from a high of $100 \mathrm{psi}$ to a low of 90 psi. All readings were within the $81-110(90-100$ with $10 \%$ margin) psi acceptance criteria range stated on page 9 of the operational test procedure and is therefore acceptable. This data is shown graphically in section 4.1 and copies of the actual data sheets generated during the test are contained in section 8 of this test report.

\section{GLY-PI-801 "GLY Pp SUCT PRESS"}

The recorded readings for the glycol suction pressure during testing of SA-CMP-801 ranged from a high of $9.5 \mathrm{psi}$ to a low of approximately $2.5 \mathrm{psi}$. All readings were within the $0-10(0-11$ with $10 \%$ margin) psi acceptance criteria range stated on page 9 of the operational test procedure and is therefore acceptable. This data is shown graphically in section 4.1 and copies of the actual data sheets generated during the test are contained in section 8 of this test report.

\section{GLY-PI-803 "GLY Pp DISCH PRESS"}

The recorded readings for glycol pump 802 discharge pressure during testing of SA-CMP-801 ranged from a high of $63 \mathrm{psi}$ to a low of approximately $57 \mathrm{psi}$. Some of these readings were outside the 58.5 . $82.5 \mathrm{psi}$ (65-75 psi with $10 \%$ margin) acceptance criteria range stated on page 9 of the operational test procedure. This represents an approximate $12 \%$ margin rather than the $10 \%$ margin stated on page 9 of the OTP. Possible explanations for not meeting the acceptance criteria margin could have resulted from glycol pump 802 having a lower overall efficiency than pump 801 (used satisfactorily during testing of SA-CMP-802 below), a slightly smaller impeller, a slightly oversized impeller casing, a small amount of recirculation in the impeller cusing, or a very minor intermittent bearing problem causing the pump to run slower. All of which would cause lower discharge heads and flows. It must be noted however that during testing of $\mathrm{SA}-\mathrm{CMP}^{3}-801$ with glycol pump 802 all temperature and pressure parameters were maintained; system performance did not deteriorate due to inadequate coolant flow. It is not believed that the glycol pump discharge readings are indicative of an inferior design but rather system anomalies that will be monitored during full time operation of the system and should not be construed as failing to meet acceptance criteria. Work package $2 \mathrm{E}-96-1078$ is being prepared to either replace the pump motor or bearings as required after full time operation commences and more information regarding system performance is obtained. This data is shown graphically in section 4.1 and copies of the actual data sheets generated during the test are contained in section 8 of this test report.

\section{IA-PI-818 "IA TWR 802 PRESS" and IA-PI-819 "IA TWR 801 PRESS"}

The recorded readings for the instrument air drying tower pressures during testing of SA-CMP-801 ranged from a high of $105 \mathrm{psi}$ to a low of approximately $90 \mathrm{psi}$. Some of the readings were recorded as 0 (zero) psi due to the fact that the reading was taken while the tower was off line during the automatic switching function. This is not an indication of a faulty dryer, but a sign of normal operation. All readings were within the $80-105(72-115.5$ with $10 \%$ margin $)$ psi acceptance criteria range stated on page 9 
HNF-SD-WM-OTR-231, Rev 0

Page 5 of 165

of the operational test procedure and is therefore acceptable. This data is shown graphically in section 4.1 and copies of the actual data sheets generated during the test are contained in section 8 of this test report.

\section{GLY-TI-806 "HX-801 \& HX-802 OUTLET TEMP"}

The recorded readings for the heat exchanger outlet temperature during testing of SA-CMP-801 ranged from a high of $105 \mathrm{~F}$ to a low of approximately $79 \mathrm{~F}$. Some of the readings were outside the $90-132 \mathrm{~F}$ (100-120 F with $10 \%$ margin) acceptance criteria range stated on page 9 of the operational test procedure. This is not an indication of a faulty heat exchanger, but a result of ambient conditions being much cooler than expected and an ambient temperature range being wider than expected. The heat exchanger outlet temperature range was selected based on data sheets used during the summer of 1996 and this Operational Test was performed during March of 1997. This data is shown graphically in section 4.1 and copies of the actual data sheets generated during the test are contained in section 8 of this test report. 
4.1 GRAPHS OF RECORDED TEST DATA FOR SA-CMP-801
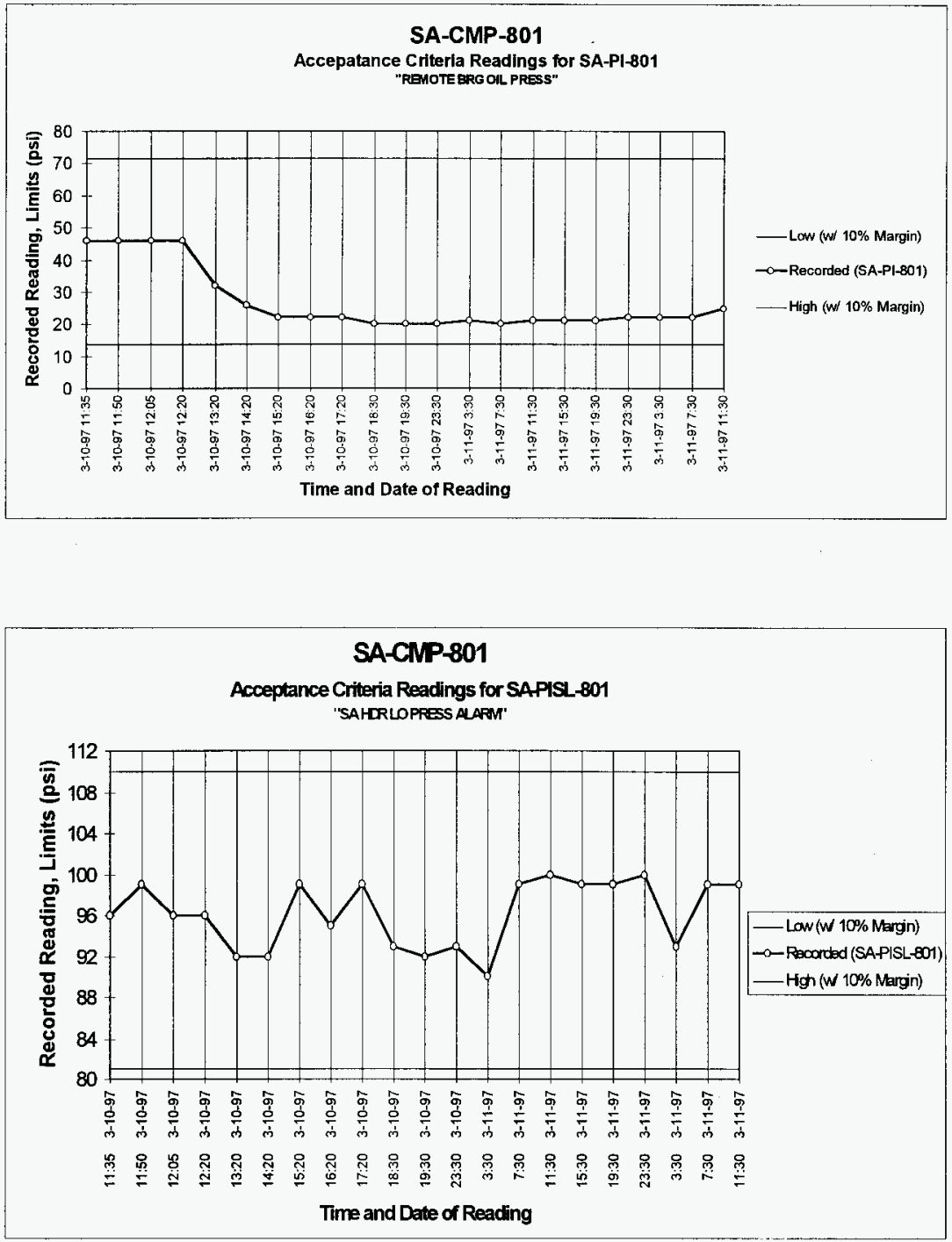

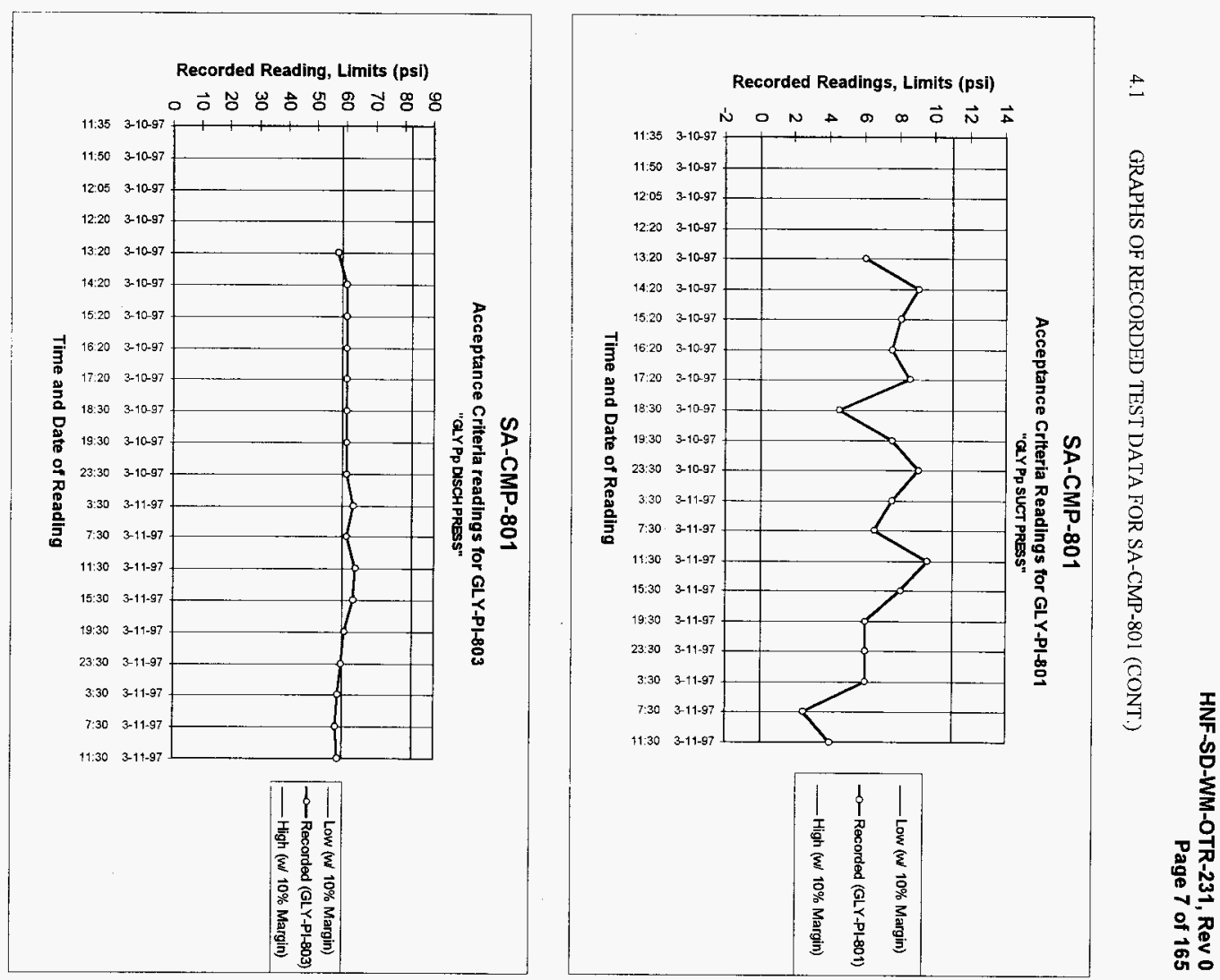
4.1 GRAPHS OF RECORDED TEST DATA FOR SA-CMP-801 (CONT.)
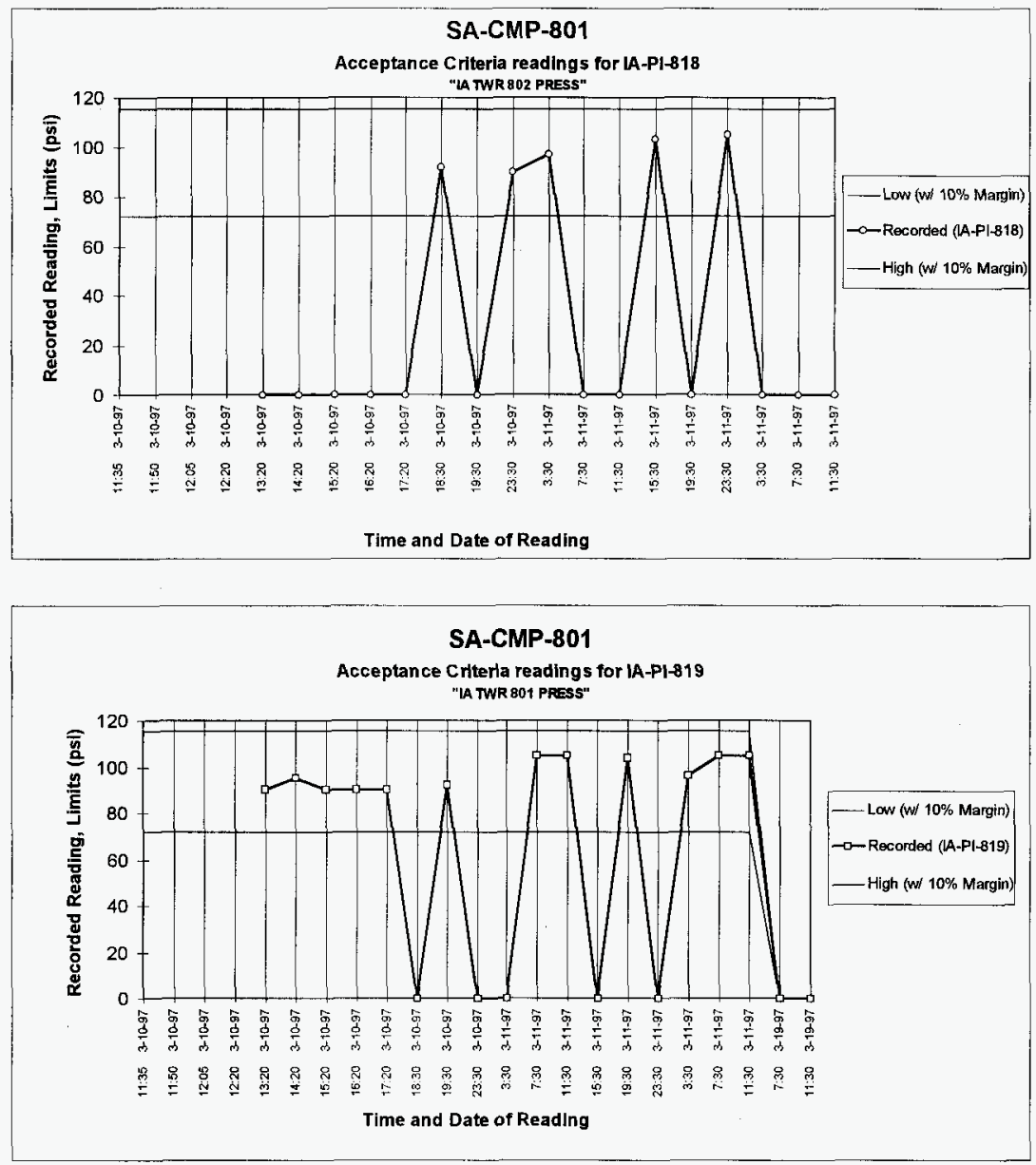
HNF-SD-WM-OTR-231, Rev 0

Page 9 of 165

4.1 GRAPHS OF RECORDED TEST DATA FOR SA-CMP-80I (CONT.)

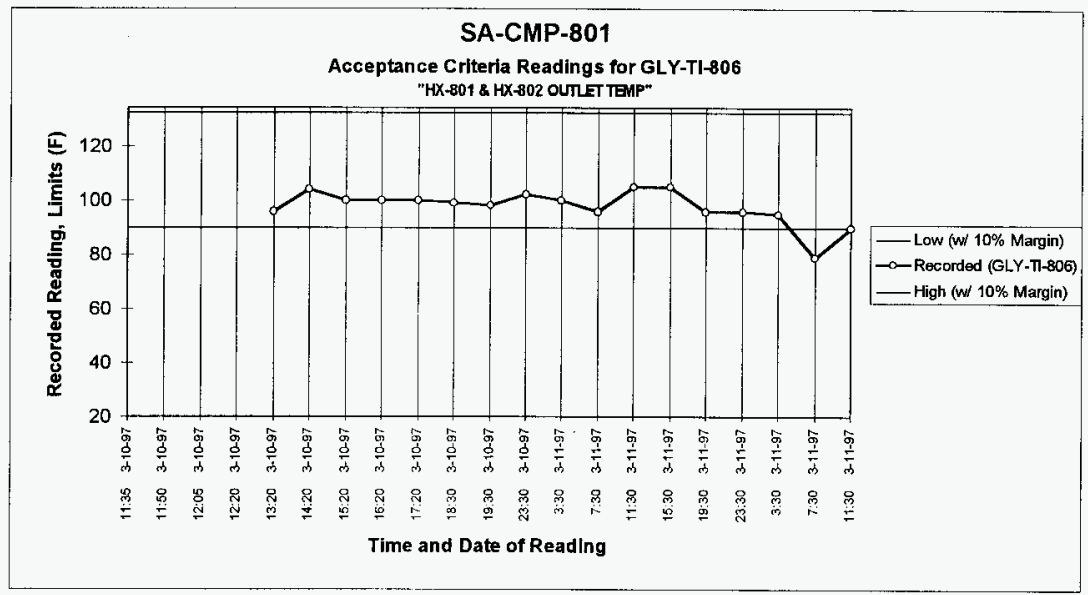


The remaining instruments and their readings which were used for meeting the acceptance criteria during testing of SACMP-802 are listed below:

\section{SA-PI-802 "REMOTE BRG OIL PRESS"}

The recorded readings for oil pressure on SA-CMP-802 ranged from a high of 48 psi at the start of the test to a low of approximately $20 \mathrm{psi}$. All readings were well within the $13.5-71.5(15-65$ with $10 \%$ margin) psi acceptance criteria range stated on page 9 of the operational test procedure and is therefore acceptable. This data is shown graphically in section 4.2 and copies of the actual data sheets generated during the test are contained in section 8 of this test report.

\section{SA-PISL-801 "SA HDR LO PRESS ALARM"}

The recorded readings for during testing of SA-CMP-802 ranged from a high of $100 \mathrm{psi}$ to a low of 92 psi. All readings were within the $81-110(90-100$ with $10 \%$ margin) psi acceptance criteria range stated on page 9 of the uperational test procedure and is therefore acceptable. This data is shown graphically in section 4.2 and copies of the actual data sheets used during the test are contained in section 8 of this test report.

\section{GLY-PI-801 "GLY Pp SUCT PRESS"}

The recorded readings for the glycol suction pressure during testing of SA-CMP-802 ranged from a high of $10 \mathrm{psi}$ to a low of approximately $1 \mathrm{psi}$. All readings were within the $0-11(0-10$ with $10 \%$ margin) psi acceptance criteria range stated on page 9 of the operational test procedure and is therefore acceptable. This data is shown graphically in section 4.2 and copies of the actual data sheets generated during the test are contained in section 8 of this test report.

\section{GLY-PI-803 "GLY Pp DISCH PRESS"}

The recorded readings for the glycol discharge pressure during testing of SA-CMP-802 ranged from a high of 68 psi to a low of approximately $60 \mathrm{psi}$. All readings were within the $58.5-82.5 \mathrm{psi}(65-75 \mathrm{psi}$ with $10 \%$ margin) acceptance criteria range stated on page 9 of the operational test procedure and is therefore acceptable. This data is shown graphically in section 4.2 and copies of the actual data sheets generated during the test are contained in section 8 of this test report.

\section{IA-PI-818 "IA TWR 802 PRESS" and IA-PI-819 "IA TWR 801 PRESS"}

The recorded readings for the instrument air drying tower pressures during testing of SA-CMP-802 ranged from a high of $105 \mathrm{psi}$ to a low of approximately $90 \mathrm{psi}$. Some of the readings were recorded as 0 (zero) psi due to the fact that the reading was taken while the tower was off line during the automatic switching function. This is not an indication of a faulty dryer, but a sign of normal operation. All readings were within the $72-115.5(80-105$ with $10 \%$ margin) psi acceptance criteria range stated on page 9 of the operational test procedure and is therefore acceptable. This data is shown graphically in section 4.2 and copies of the actual data sheets generated during the test are contained in section $\mathbf{8}$ of this test report.

\section{GLY-TI-806 "HX-801 \& HX-802 OUTLET TEMP"}

The recorded readings for the heat exchanger outlet temperature during testing of SA-CMP-801 ranged from a high of $86 \mathrm{psi}$ to a low of approximately $60 \mathrm{psi}$. All readings were outside the $90-132 \mathrm{~F}$ (100-120 
HNF-SD-WM-OTR-231, Rev 0

Page 11 of 165

F with $10 \%$ margin) acceptance criteria range stated on page 9 of the operational test procedure. This is not an indication of a faulty heat exchanger, but a result of ambient conditions being much cooler than expected. The heat exchanger outlet temperature range was selected based on data sheets used during the summer of 1996 and this Operational Test was performed during March of 1997 . This data is shown graphically in section 4.2 and copies of the actual data sheets generated during the test are contained in section 8 of this test report. 


\subsection{GRAPHS OF RECORDED TEST DATA FOR SA-CMP-802}
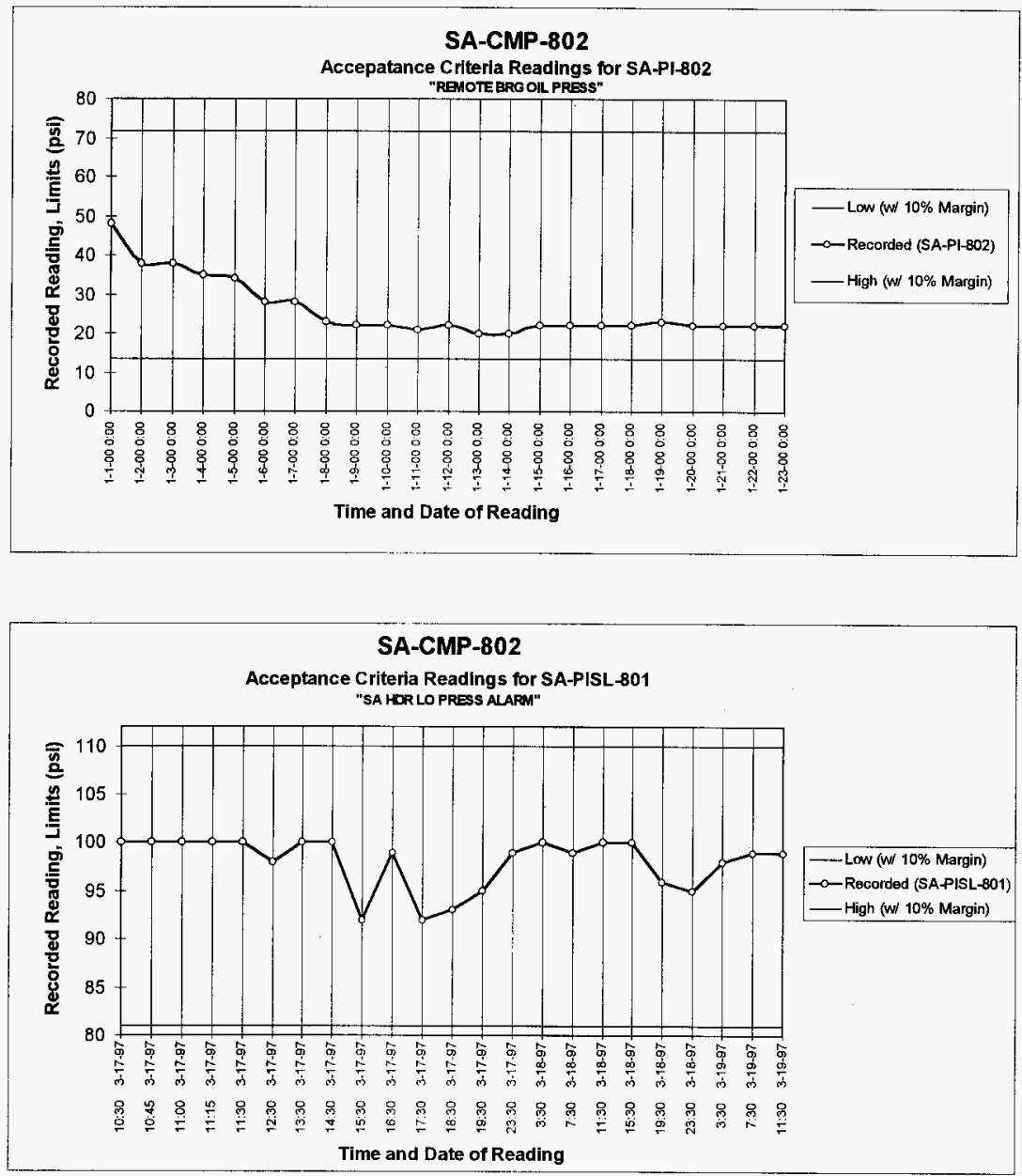


\subsection{GRAPHS OF RECORDED TEST DATA FOR SA-CMP-802 (CONT.)}
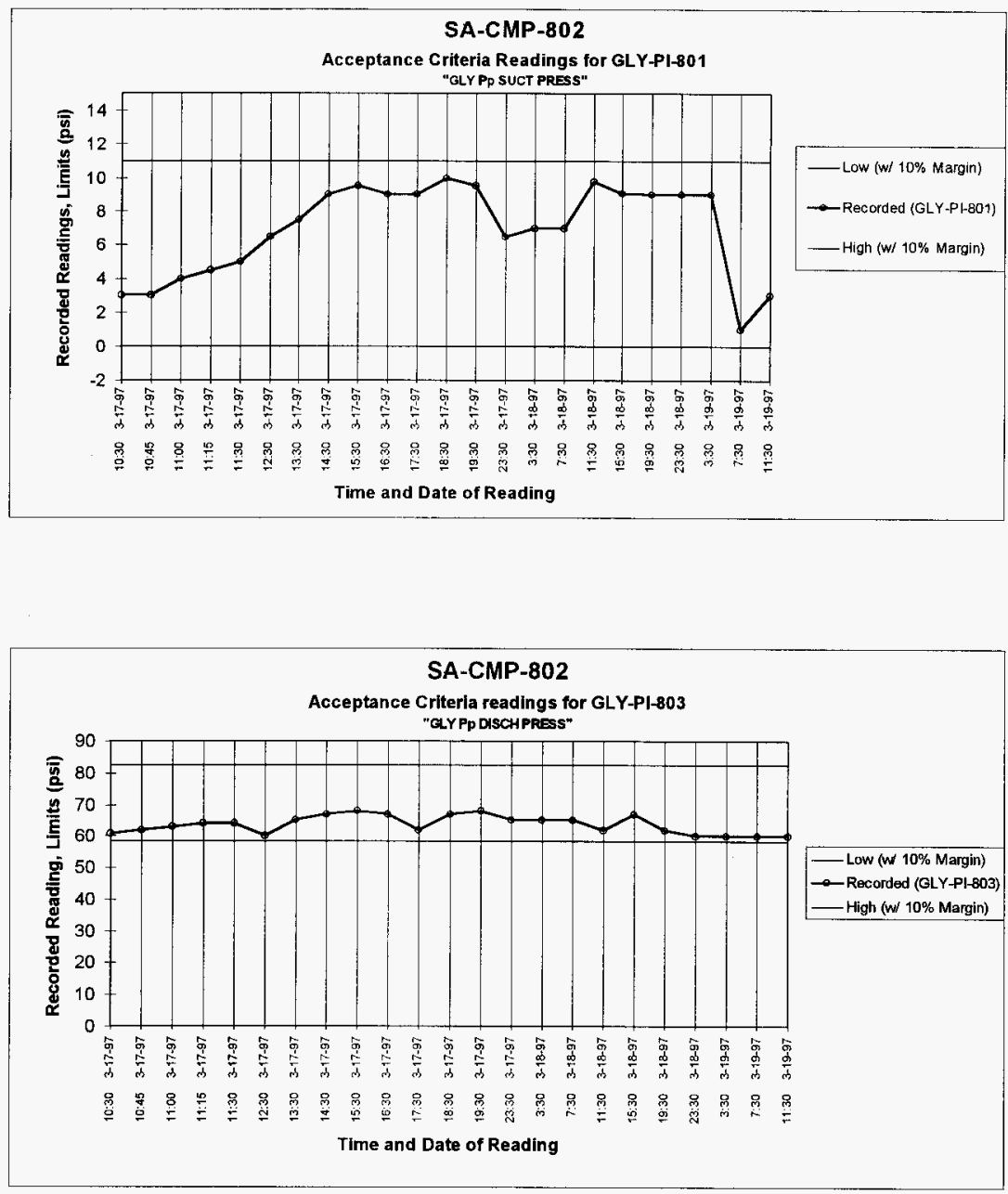
4.2 GRAPHS OF RECORDED TEST DATA FOR SA-CMP-802 (CONT)
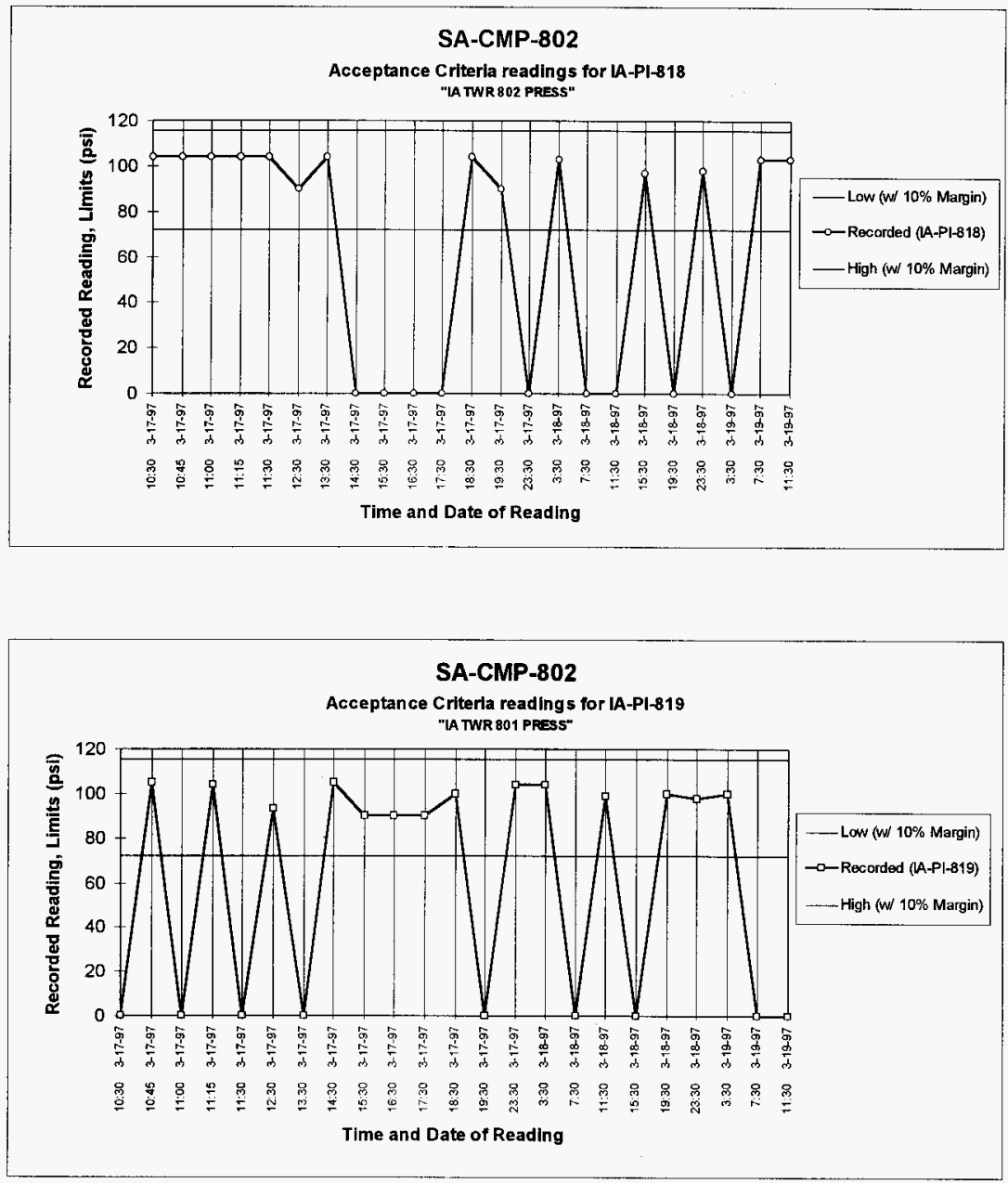
4.2 GRAPHS OF RECORDED TEST DATA FOR SA-CMP-802 (CONT.)

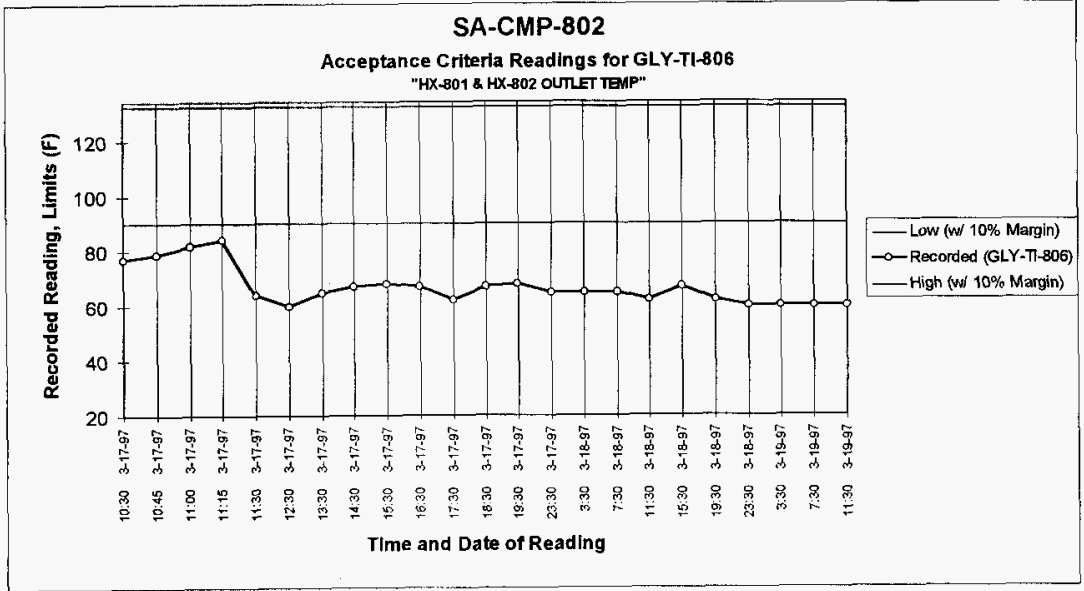


After completion of the tests for both SA-CMP-801 and SA-CMP-802 it was discovered that not all of the data sheets could be located.

During testing of SA-CMP-801 data sheets (page 33 of OTP-200-003) covering the dryer and cooling skid operation during the first hour of testing could not be located. Data sheets (page 31 and 33 of OTP-200-003) covering SA-CMP-801 as well as the dryer and cooling skid operation at the end of the one hour data taking interval could also not be located. The associated data sheets that could be located for the same periods as those mentioned above (i.e. page 31 of OTP-200-003) showed that the system was operating normally. Trending the data on the recovered data sheets did not show any evidence of abnormalities or any cause for concern. A summary of the data sheets which were generated during testing of SA-CMP-801 can be found in section 5.1 of this report. 
HNF-SD-WM-OTR-231, Rev 0

Page 17 of 165

\subsection{SA-CMP-801 DATA SHEET SUMMARY}

\begin{tabular}{|c|c|c|c|}
\hline Data Taking Interval & $\begin{array}{l}\text { Expected Time And Dute That } \\
\text { Data Is To Be Taken }\end{array}$ & $\begin{array}{l}\text { Actual Time And Date That Data } \\
\text { Wus Taken }\end{array}$ & $\begin{array}{l}\text { Data Shect Pages From } \\
\text { OTP-200-003 Accounted For }\end{array}$ \\
\hline $15 \mathrm{~min}$ & $11: 35 \quad 3 / 10 / 97$ & $11: 35 \quad 3 / 10 / 97$ & page $31, * * *$ \\
\hline $15 \mathrm{~min}$ & $11: 50 \quad 3 / 10 / 97$ & $11: 50 \quad 3 / 10 / 97$ & page $31, * * *$ \\
\hline $15 \mathrm{~min}$ & $12-05 \quad 3 / 10 / 97$ & $12: 05 \quad 3 / 10 / 97$ & page $31, * * *$ \\
\hline $15 \mathrm{~min}$ & $12: 20 \quad 3 / 10 / 97$ & $12: 20 \quad 3 / 10 / 97$ & page $31, * * *$ \\
\hline $1 \mathrm{hr}$ & $13: 20 \quad 3 / 10 / 97$ & $13: 20 \quad 3 / 10 / 97$ & page 31 , page 33 \\
\hline $1 \mathrm{hr}$ & $14: 20 \quad 3 / 10 / 97$ & 14:20 $3 / 10 / 97$ & pagc 31 , page 33 \\
\hline $1 \mathrm{hr}$ & $15: 20 \quad 3 / 10 / 97$ & $15: 20 \quad 3 / 10 / 97$ & page 31 , page 33 \\
\hline $1 \mathrm{hr}$ & $16: 20 \quad 3 / 10 / 97$ & $16: 30 \quad 3 / 10 / 97$ & page 31 , paye 33 \\
\hline $1 \mathrm{lu}$ & $17: 21+3 / 16 \% 7$ & $17: 20 \quad 3 / 10 / 97$ & page 31 , page 33 \\
\hline $1 \mathrm{hur}$ & $1830303 / 10 / 97$ & $18: 30 \quad 3 / 10 / 97$ & page 31 , page 33 \\
\hline $1 \mathrm{hr}$ & 19:30 3/1(1/97) & 19:30 3/10/97 & page 31 , page 33 \\
\hline $1 \mathrm{hr}$ & $20: 30 \quad 3 / 10 / 97$ & Should Have Becn 20:30 3/10/97 & $*, * * *$ \\
\hline $4 \mathrm{hr}$ & $00: 303 / 10 / 97$ & $23: 30 \quad 3 / 10 / 97$ & page 31 , page 33 \\
\hline $4 \mathrm{hr}$ & $04: 30 \quad 3 / 11 / 97$ & $03: 303 / 11 / 97$ & pagc 31 , pagc 33 \\
\hline $4 \mathrm{hr}$ & $08: 30 \quad 3 / 11 / 97$ & $07: 30 \quad 3 / 11 / 97$ & page 31 , page 33 \\
\hline $4 \mathrm{hr}$ & $12: 303 / 11 / 97$ & $11: 30 \quad 3 / 11 / 97$ & page 31 , page 33 \\
\hline $4 \mathrm{hr}$ & 16:31) 3/11/97 & $15: 303 / 11 / 97$ & page 31 , page 33 \\
\hline $4 \mathrm{hr}$ & $20: 30 \quad 3 / 11 / 97$ & $19: 303 / 11 / 97$ & page 31 , page 33 \\
\hline $4 \mathrm{hr}$ & $00: 30 \quad 3 / 11 / 97$ & $23: 30 \quad 3 / 11 / 97$ & page 31 , page 33 \\
\hline $4 \mathrm{hr}$ & $04: 30 \quad 3 / 12 / 97$ & $03: 30 \quad 3 / 12 / 97$ & page 31 , page 33 \\
\hline $4 \mathrm{hr}$ & $08: 30 \quad 3 / 12 / 97$ & $07: 30 \quad 3 / 12 / 97$ & page 31 , page 33 \\
\hline $4 \mathrm{hr}$ & $12: 30 \quad 3 / 12 / 97$ & $11: 30 \quad 3 / 12 / 97$ & page 31 , page 33 \\
\hline
\end{tabular}

* Copy of data sheet Page 31 oould not be found

** Copy of data sheet Page 32 could not be found

*** Copy of data sheet Page 33 could not the found 
During testing of SA-CMP-802, two sets of data sheets (page 32 and 33 of OTP-200-003) were recovered for the same time interval for data collection at 16:30 3/17/97 as well as 17:30 on 3/17/97. In addition, no data sheets were recovered for the time interval for data collection at 18:30 on $3 / 17 / 97$ as well as 19:30 3/17/97. It appears that one set of the duplicate data sheets recorded for "16:30" or "17:30" belongs to either "18:30" or "19:30". The recorded data was analyzed for trends and one set of the "duplicate" data sheets was assigned to each of the missing time intervals. This accounted for all the data sheets during testing of SA-CMP-802. A summary of the data sheets generated during testing of SA-CMP-802 can be found in section 5.2 of this report. 
HNF-SD-WM-OTR-231, Rev 0

Page 19 of 165

SA-CMP-802 DATA SHEET SUMMARY

\begin{tabular}{|c|c|c|c|}
\hline Data Taking Interval & $\begin{array}{l}\text { Expected Time and Date That } \\
\text { Data is To Be Taken }\end{array}$ & $\begin{array}{l}\text { Actual Time atd Date That Data } \\
\text { Was Taken }\end{array}$ & $\begin{array}{l}\text { Data Sheet Pages From } \\
\text { OTP-200-003 Aocounted For }\end{array}$ \\
\hline $15 \mathrm{~min}$ & $10: 30 \quad 3 / 17 / 97$ & $10: 30 \quad 3 / 17 / 97$ & Page 32 , Page 33 \\
\hline $15 \min$ & $10: 45 \quad 3 / 17 / 97$ & $10: 45 \quad 3 / 17 / 97$ & Page 32, Pagc 33 \\
\hline $15 \mathrm{~min}$ & $11: 00 \quad 3 / 17 / 97$ & $11: 00 \quad 3 / 17 / 97$ & Puge 32, Page 33 \\
\hline $15 \mathrm{~min}$ & $11: 15 \quad 3 / 17 / 97$ & $11: 15 \quad 3 / 17 / 97$ & Page 32, Page 33 \\
\hline $15 \mathrm{~min}$ & $11: 30 \quad 3 / 17 / 97$ & $11: 30 \quad 3 / 17 / 97$ & Page 32, Page 33 \\
\hline $1 \mathrm{hr}$ & 12:30 $\quad 3 / 17 / 97$ & $12: 30 \quad 3 / 17 / 97$ & Page 32, Page 33 \\
\hline $1 \mathrm{hr}$ & $13: 30 \quad 3 / 17 / 97$ & $13: 30 \quad 3 / 17 / 97$ & Page 32 , Page 33 \\
\hline $1 \mathrm{hr}$ & $14: 30 \quad 3 / 17 / 97$ & $14: 30 \quad 3 / 17 / 97$ & Page 32, Page 33 \\
\hline $1 \mathrm{hr}$ & $15: 30 \quad 3 / 17 / 97$ & $15: 30 \quad 3 / 17 / 97$ & Pagc 32, Pagc 33 \\
\hline $1 \mathrm{hr}$ & $16: 30 \quad 3 / 17 / 97$ & $16: 30 \quad 3 / 17 / 97$ & $\begin{array}{l}\text { Page } 32 \text { (2 sets) } \\
\text { Page } 33 \text { (2 sets) }\end{array}$ \\
\hline $1 \mathrm{hr}$ & $17: 30 \quad 3 / 17 / 97$ & $17: 30 \quad 3 / 17 / 97$ & $\begin{array}{l}\text { Psge } 32 \text { (2 sets) } \\
\text { Pege } 33 \text { (2 sets) }\end{array}$ \\
\hline $1 \mathrm{hr}$ & $18: 30 \quad 3 / 17 / 97$ & $\begin{array}{l}\text { Should Have Been 18:30 } \\
3 / 17 / 97\end{array}$ & $* *, * * *$ \\
\hline $1 \mathrm{hr}$ & $19: 30 \quad 3 / 17 / 97$ & $\begin{array}{l}\text { Should Have Been 19:30 } \\
3 / 17 / 97\end{array}$ & $* *, * * *$ \\
\hline $4 \mathrm{hr}$ & $23: 30 \quad 3 / 17 / 97$ & $23: 30 \quad 3 / 17 / 97$ & Page 32. Page 33 \\
\hline 4 hr & $03: 30 \quad 3 / 18 / 97$ & $03 ; 30 \quad 3 / 18 / 97$ & Page 32, Page 33 \\
\hline $4 \mathrm{hr}$ & $07: 30 \quad 3 / 18 / 97$ & $07,30 \quad 3 / 18 / 97$ & Page 32, Prge 33 \\
\hline $4 \mathrm{hr}$ & $11: 30 \quad 3 / 18 / 97$ & $11: 30 \quad 3 / 18 / 97$ & Page 32, Page 33 \\
\hline $4 \mathrm{hr}$ & $15: 30 \quad 3 / 18 / 97$ & $15: 30 \quad 3 / 18 / 97$ & Pugc 32, Page 33 \\
\hline $4 \mathrm{hr}$ & $19: 30 \quad 3 / 18 / 97$ & $19: 30 \quad 3 / 18 / 97$ & Page 32, Page 33 \\
\hline $4 \mathrm{hr}$ & $23: 30 \quad 3 / 18 / 97$ & $23: 30 \quad 3 / 18 / 97$ & Page 32, Page 33 \\
\hline $4 \mathrm{hr}$ & $03: 30 \quad 3 / 19 / 97$ & $03: 30 \quad 3 / 19 / 97$ & Page 32, Page 33 \\
\hline $4 \mathrm{hr}$ & $07: 30 \quad 3 / 19 / 97$ & $07: 30 \quad 3 / 19 / 97$ & Page 32, Page 33 \\
\hline $4 \mathrm{hr}$ & $11: 30 \quad 3 / 19 / 97$ & $10: 30 \quad 3 / 19 / 97$ & Page 32, Page 33 \\
\hline
\end{tabular}

* Copy of data sheet Page 31 could not be found

** Copy of data sheet Page 32 could not he found

*** Copy of dats sheet Page 33 could not he found 
The OTP was completed and signed off without major complications, however the following exceptions were noted during testing (the actual test exceptions noted during the test are included in section $\mathbf{8}$ of this test report but are paraphrased here for reference):

\section{Exception 1}

The sensing line connection for the load and unload signal for SA-CMP-801 and SA-CMP-802 is located on the air header, not the receiver tank. This causes the compressor to load and unload based on the air header pressure and not the receiver tank if the receiver tank is not valved in.

Resolution of Exceplion 1

A new step, 5. 1.21.1, was inserted into OTP-200-003 to allow for valve SA-V-825 to be opened after step 5.1.21. This allows the receiver tank and air header pressures to coincide and allows the receiver tank to be filled.

\section{Exception 2}

The valves listed in the tables asseciated with steps 5.1.39 and 5.2.4 control the air supply from the new compressors CMP-SA-801 (or CMP-SA-802) to A-Complex. The valves in the preceding steps, 5.1.38 and 5.2.3 respectively, contrul the air supply from the backup compressors. The amount of time required to perform each of these tables causes the potential to secure air to A-Complex.

Resolution of Exception 2

By reversing the order of these steps the air supply from CMP-SA-801 (or SA-CMP-802) will be valved in prior to securing the air supply from the backup compressors. Steps 5.1.39 and 5.2.4 were performed prior to steps 5.1 .38 and 5.2 .3 respectively. This allows a continuous air supply and prevents the air receiver from being drawn down after the backup compressors are valved out.

\section{Exception 3}

Closing valve SA-V-825 in step 5.2 .3 isolates receiver tank.

Resolution of Exception 3

Step 5.2.3 was moved after 5.2.12.

\section{Exception 4}

Air dryer isolation during system shutdown. The OTP contained a step to open the bypass valve and close the inlet valve. The closing of the outlet valve wasn't performed until after the dryer was attempted to be bled down.

Resolution of Exception 4

This error was correcled by moving the step for closing the dryer outlet to coincide with closing the dryer inlet, thereby isolating the dryer. 


\section{HNF-SD-WM-OTR-231, Rev 0 \\ Page 21 of 165}

\section{Exception 5}

IA-PISL-801 should be SA-PISL-801

Resolution of Exception 5

Change IA-PISI,-801 to read SA-PISL-801 in steps 5.1.28 and 5.1.54.

\section{Exception 6}

The instrument air flow indicator, IA-FI-801, readings were below expected range. The Air Dryer/Coolant Skid data sheet found on page 33 of OTP-200-002 indicated an expected range of 45 CFM to $90 \mathrm{CFM}$.

Resolution of Exception 6

This range was used based on the limited operation of the 241-A-701 compressed air system during the summer of 1996 . However, during testing the readings were less than 20 scfm which is below the low end of the scale. Possible explanations regarding the low instrument air demand include the installations of ENRAF type liquid level measurements which do not require purge air and the repair of several small leaks during the construction phase of Projects W-030, W-151, and W-320. The flow indicator was verified as operational by temporarily introducing a small load which was measured by the flow indicator.

\section{Exception 7}

Numerous signature blanks through out the operational test procedure were not completed during testing.

Resolution of Exception 7

The signatures which were not recorded during testing can be found in the body of the Operational Test Procedure (OTP) which is also included in this Operational Test Report (OTR) in Section 8. The signatures were not recorded as an oversight. During review of the test procedure after testing, it was determined that the signatures which were not obtained during formal testing of the 701-A compressor system have a negligible impact on the performance and quality of the test and do not affect the validity of the results. Examples of some of the locations of missing signatures can be found on pages 40,48, and 51 of this Operational Test Report (OTR). 


\section{HNF-SD-WM-OTR-231, Rev 0}

Page 22 of 165

\subsection{CONCLUSION}

The OTP successfully demonstrated that the compressed air system at 241-A-701 operates within its intended design parameters. System performance was monitored, recorded, and used to identify any potential area of concem. This Operational Test Report (OTR) documents the successful completion of the OTP and formalizes the transfer of ownership to Tank Farm Operations and that the system is ready for use. The official signed off copy of the "OTP ACCEPTANCE SHEET" contained in OTP-200-003 can be found in section 8, page 165 of this test report. 
HNF-SD-WM-OTR-231, Rev 0

Page 23 of 165

8.0 COMPLETED COPY OF OTP-200-003 PROCEDURE STEPS 


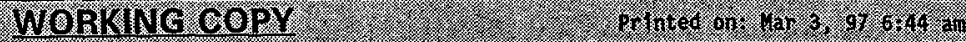

TANK FAM OPERATONAL TSST PROCEDURE A ARM OPERATIONS

\section{1-A-701 AIR COMPRESSOR OPERATIONAL TEST PROCEDURE}

The original signatures are on file.

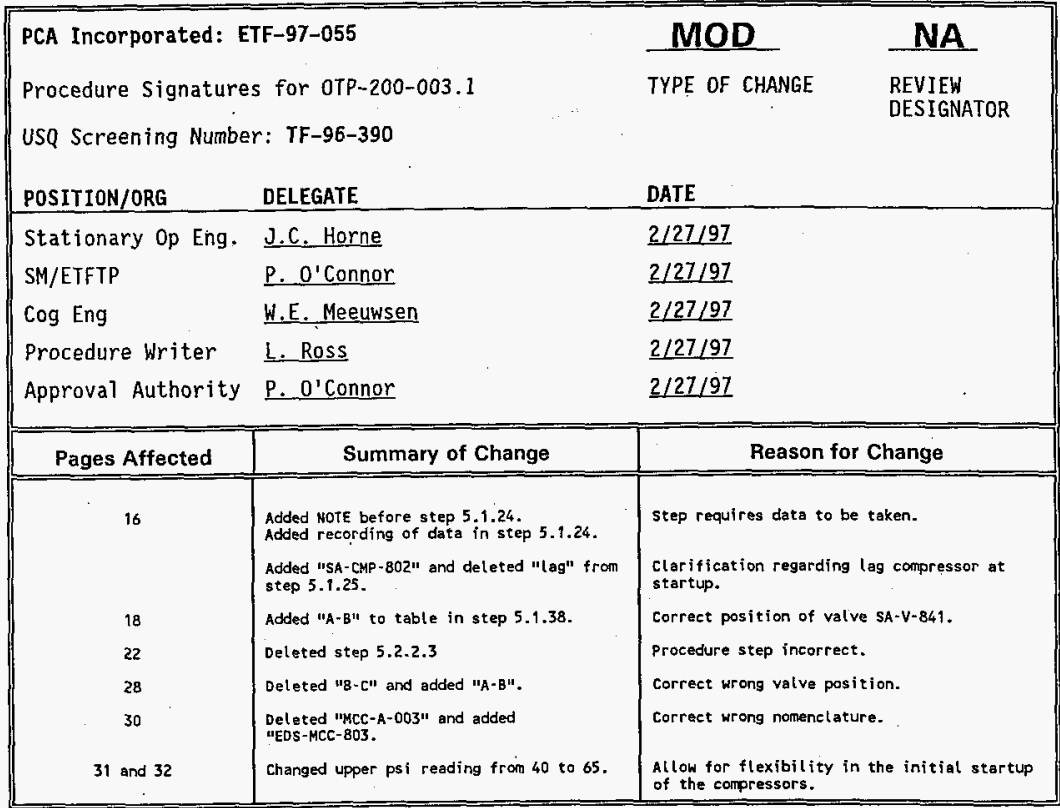


2.0. INFORMATION

2.1 SCOPE

2.2 TERMS AND DEFINITIONS

2.3 RESPONSIBILITIES

2.4 REFERENCES

\subsection{SAFETY}

2.6 RADIATION AND CONTAMINATION CONTROL

\subsection{QUALITY ASSURANCE}

2.8 GENERAL INFORMATION

2.9 LIMITATIONS AND PRECAUTIONS $\quad 10$

3.0 RECORDS

4.0 PREREQUISITES

5.0 PROCEDURE

5.1 PERFORM 48 HOUR ON-LINE TEST 14

5.2 PERFORM SYSTEM SHUTDOWN 22

PROCEDURE PERFORMER SIGNATURE SHEET 25

CHECKLIST 1 - COOLING WATER VALVING 26

CHECKLIST 2 - PROCESS AIR VALVING $\quad 27$

CHECKLIST 3 - INSTRUMENT AIR VALVING 28

CHECKLIST 4 - ACCUMULATOR INSTRUMENT AIR. VALVING • 29

$\begin{array}{lr}\text { CHECKLIST } 5 \text { - ELECTRICAL LINE-UP } & 30\end{array}$

48 HOUR ON-LINE TEST DATA SHEET

For SA-CMP-801 . 31

For SA-CMP-802 $\quad 32$

For AIR DRYER/COOLING SKID

ATTACHMENT 1 - OTP ACCEPTANCE $\quad 34$

ATTACHMENT 2 - OTP EXCEPTION LOG

$\begin{array}{ll}\text { Procedure History Signature Sheet } & 36\end{array}$ 


\subsection{PURPOSE}

The purpose of this Operational Test Procedure is to provide instructions to verify that 241-A-701 Air Compressors and associated components meet the functional, operational and design requirements with alf items in the final in-service configuration.

This Operational Test Procedure (OTP) for the 701-A Compressor Upgrade has been written and issued to replace the original test $\mathrm{plan}$ WHC-SD-WM-0TP-169. The original test plan (WHC-SD-WM-OTP-169) was completed intermittently over several months; as a result several data sheets are missing, steps in the OTP were omitted or not signed off, and portions of the system were not tested satisfactorily. This OTP procedure is based on the Operating Procedure T0-200-610 "Operate the 701-A Compressors" and the original OTP, WHC-SD-WM-0TP-169.

\subsection{INFORMATION}

\subsection{SCOPE}

2.1.1 This test involves running the two Ingersoll-Rand Air Compressors and associated components at the 241-A-701 building.

2.1.2 The activities defined in this OTP will be performed to ensure the daily operation of the new compressed air system can be reliable and efficient.

2.1.3 Completion of this OTP and sign off of the OTP Acceptance of Test Results (Attachment 1) is necessary for turnover of the compressed air system to Lockheed Mart in Hanford Company (LMHC) Operations.

\subsection{TERMS AND DEFINITIONS}

VERIFY - Perform a comparison with the specified requirements. Does not involve manipulation of equipment or instrumentation. May be done by methods other than direct observation.

ENSURE - To confirm, substitute, and assure that an activity or condition has been implemented in conformance with the specified requirements or take action to make it so if the activity or condition is not found to be in conformance. May be done by methods other than direct observation.

The prefix 701-A has been dropped from equipment and labels in all tables and text, for the purpose of this document.

ATTENDED - Present in building during test.

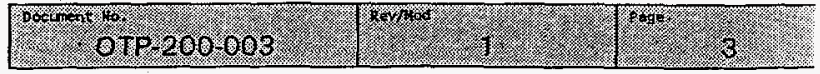




\subsection{RESPONSIBILITIES}

\subsubsection{FDH Craft Personnel}

FDH Craft Personnel shall be in STANDBY for this OTP. With the approval of the Test Director, they shall provide resolution to test exceptions or complete any other needed repairs/rework of the system.

\subsubsection{LMHC Facility Operations}

The operations representative, who shall be referred to herein as the Test Director (TD), is the individual in charge during this OTP and is responsible for the following:

- Assuring the test is performed in accordance with this document

- Coordinating and overseeing the performance of the test

- Initialing the completion of each indicated step

- Initiating Procedure Change Authorizations to this procedure as needed.

- Accepting results, resolving exceptions and contacting the appropriate personnel to witness hold points.

2.3.3 Quality Assurance (QA)

Quality Assurance is responsible for reviewing and approving exception resolutions as determined by the Test Director; for reviewing and accepting the test results.

\subsubsection{Quality Control (QC)}

Quality Control is responsible for reviewing and approving this procedure as determined by the Test Director; for reviewing and accepting $Q C$ hold point steps.

\subsubsection{LMHC Design Engineers}

Design Engineers are responsible for resolving any engineering or technical problems associated with this OTP, as requested by the Test Director or System Cognizant Engineer, for successful completion of this OTP. 


\subsection{RESPONSIBILITIES (Cont.)}

\subsubsection{System (Electrical or Mechanical) Cognizant Engineer}

The System Cognizant Engineer or designee, is responsible for performing a walkdown of the systems related to this Operational Test Procedure; for reviewing and approving exception resolutions; for accepting the test results; for issuing the Operation Test Report.

\subsubsection{FDH Stationary Operating Engineers}

Shall assist in performance of the test as required and are responsible for recording test data.

\subsection{REFERENCES}

2.4.1 The following documents were used to write or are referenced in this procedure:

- CVI \#22172, Vendor Information

- WHC-IP-0842, WASTE TANK ADMINISTRATION, Vol. 2 , Section 4.9.1, "Lockout/Tagout"

- WHC-CM-6-j, STANDARD ENGINEERING PRACTICES, EP-4:2, "Testing Requirements"

- WHC-SD-WM-HSP-002, HEALTH AND SAFETY PLAN, Safe Work Practices

- WHC-CM-1-10, Safety Manual

- WHC-CM-1-11, Industrial Hygiene Manual

- HSRCM, Hanford Site Radiological Control Manual

- WHC-IP-0263, BUILDING EMERGENCY PLAN

- WHC-SD-WM-ATP-083, Acceptance Test Procedure

- T0-200-610, OPERATE THE 701-A COMPRESSORS

2.4.2 The following drawings were used to write or are referenced in this procedure:

- $\mathrm{H}-2-62895$

- $\mathrm{H}-2-85093$

- $\mathrm{H}-2-85277$

- $\mathrm{H}-2-85300$

- $\mathrm{H}-14-020308$

- H-14-020708

- SK-2-24096

- $\quad$ SK-2-24098 


\subsection{SAFETY}

2.5.1 If the performance of this procedure is suspended for any reason, ensure the equipment is left in a safe state.

2.5.2 This test will be conducted on a pressurized system. The TD sha1l ensure that protective guards are in place before starting any part of the OTP, and personnel not conducting the test remain clear.

2.5.3 The compressors shall not be left running unattended. In the event that any part of the OTP should be suspended, the system shall be shutdown then placed in a safe condition as determined by the TD, before it is left unattended.

2.5.4 All lockouts and tagouts or over-tagging requirements shall be performed in accordance with Tank Farm Administration Manua7, WHC-IP-0842, Vol 2, Section 4.9.1, Lockout/Tagout.

2.5.5 If any equipment problem is observed during the performance of this procedure, immediately notify the Test Director and Shift Manager.

2.5.6 Hearing protection is required in the 241-A-701 compressor building.

2.5.7 All personnel shall be aware of hazardous materials used during this test, and location of the MSDS for each substance.

2.5.8 Eye protection must be worn during compressor start-up.

2.5.9 The following documents define the limits and restrictions to perform this procedure:

- Safety Manual, WHC-CM-1-10

- Building Emergency PIan, WHC-IP-0263TF

- Industrial Hygiene Manual, WHC-CM-1-11

- Environmental Compliance Manual, WHC-CM-7-5

- Tank Farm Health and Safety Plan (HASP), WHC-SD-WM-HSP-0O2 
-(568Z9-Z-H 6utmesp aәs) $\varepsilon-20 L-\Lambda$ pue $t-20 L-A$ ' $2-20 L-A$

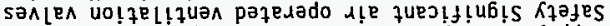

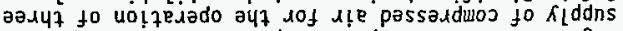

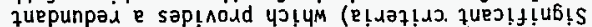

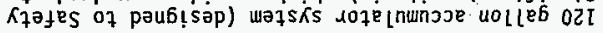

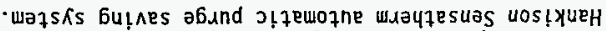
e 47 !M padd!nba s! laKup aप1 qu!od map $y_{0} 0 t^{-}$

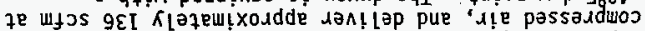

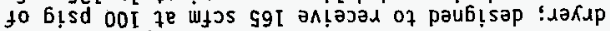
ג!e passadduos quejo!sap $59[-H O$ ssalfeay uos!

$\cdot I 0 L-\forall-I b Z$ 6u!pl!nq ap!su! pazejol a.de yue a6uns pue

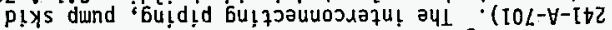

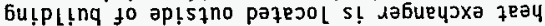

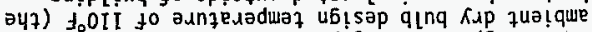

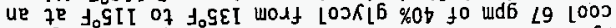

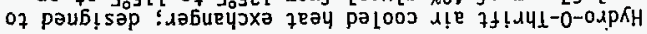

- suojesedas asnzstow pue dajoosdazje

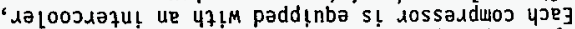

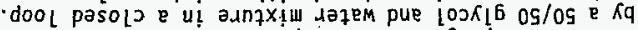

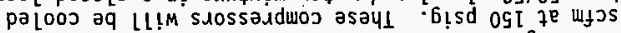

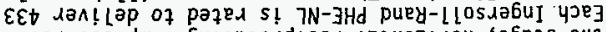
suossalduos bu!zesoldijad lequoz!loy 'abezs om7

' $7 N-\exists H d$ pueb-LlosiabuI (dH) jamodasioh SZI mau OMI

:apn!วu!

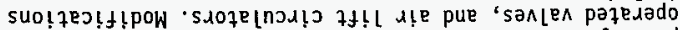

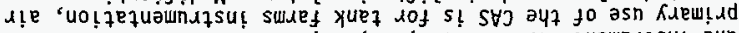

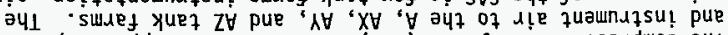

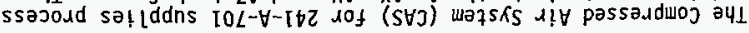

\section{NOIIdIYJS30 WJISAS ['8*2}

\section{NOIL $\forall W Y O A N I$ T $74 \exists N \exists 98 \cdot \tau$}

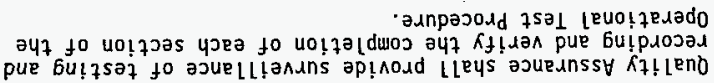

\section{ヨON}

- seaje lejtbolomped

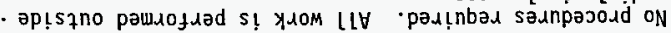

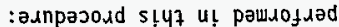

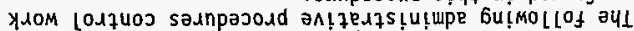

$[\cdot 9 \cdot 2$

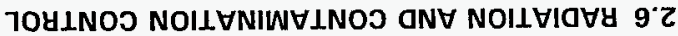




\section{WORKING COPY}

\subsection{GENERAL INFORMATION (Cont.)}

\subsubsection{TEST DATA RECORDING INSTRUCTIONS}

\section{Exception Sheets}

Throughout this procedure, spaces are provided for the TD initials. Where required, additional spaces will be provided for $L M H C$ COG, and QC initials.

Any exceptions (deviations from design requirements, or stated operational 'limits) that arise during the test will be recorded on an OTP Exception Log (Attachment 2), and the exception number shall be noted next to the applicable procedure step. Exception Sheets are not needed for steps that indicate rework or recovery instructions. Exception resolutions will include appropriate resolution for each exception. Each exception shall be approved by the TD and COG. LMHC QA shall review and approve each exception if requested by the TD; their approval may be obtained at the time the resolution is written. The TD shall decide whether to cont inue the test after the discovery of the exception. The test shall not continue without resolving and repairing an exception unless the TD determines the exception will not jeopardize personne?, equipment, or invalidate the test results.

\section{Procedure Modification}

If the OTP requires modification due to unforseen circumstances, the Systems Cognizant Engineer shall make a Procedure Change Authorization (PCA) change to the OTP Working Copy.

Note - Acceptance for Use, Operability Test Report and Acceptance for Beneficial Use, must be completed before turn-over to Operations.

Acceptance for Use

When the Shift Operations Manager and the East Tank Farms Transition Project Manager have verified that the OTP has been completed, they shall sign in the spaces provided on the Acceptance of Test Results Sheet (Attachment 1) accepting the 241-A-701 Instrument Compressed Air System "for use".

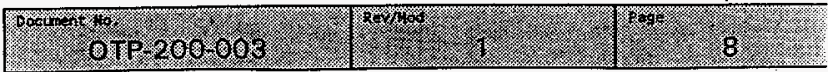




\subsection{GENERAL INFORMATION (Cont.)}

Operability Test Report

A copy of the completed test shall be forwarded to the Systems Cognizant Engineer. This data will be used to prepare the Operability Test Report. The Record Copy of the OTP will be placed in the LMHC Work Package. The Systems Cognizant Engineer will be responsible for obtaining all signatures on the Acceptance of Test Results and sending a signed copy to each responsible individual.

Acceptance for Beneficial Use (ABU)

The ABU will be completed by the Facility Systems Engineer with a tentative completion date of February, 1997.

2.8.3 Acceptance criteria for the 701-A compressors will include the following:

1. Successful completion of the 48 hour operation of each compressor without interruption.

2. Ability of the compressed air system to supply air to the $A, A X, A Y$, and $A Z$ tank farms. This criteria will be measured by successful 48 hour continuous running with completed data sheet entries for the following instruments:

Compressors:

SA-PI-801 "REMOTE BRG OIL PRESS"

SA-PI-802 "REMOTE BRG OIL PRESS"

SA-PISL-801 "SA HDR LO PRESS ALARM"

Coolant Skid/Heat Exchanger:

GLY-PI-801 "GLY Pp SUCT PRESS"

GLY-PI-803 "GLY PP DISCH PRESS"

GLY-FG-802 "SA-CMP-801 HP SIDE GLY FLO"

GLY-FG-806 "SA-CMP-802 HP SIDE GLY FLO"

GLY-TI-806 "HX-801 \& HX-802 OUTLET TEMP"

Air Dryers:

IA-PI-819 "IA TWR 801 PRESS"

IA-PI-818 "IA TWR 802 PRESS"

IA-FI-801 "IA FLOW INDICATOR"

The remaining data recorded on 48 HOUR ON-LINE TEST Data Sheets, are for informational purposes only. All readings, including informational as wel1 as acceptance criteria, must be within $10 \%$ of the expected range as delineated on 48 HOUR ONLINE TEST Data Sheets.

IF specific acceptance criteria stated in this OTP are not met IHEN this OTP will be suspended until all nonconformance(s) are corrected. 


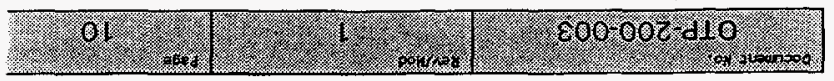

Siqua.

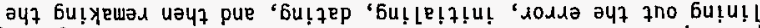

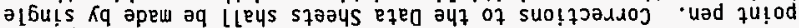

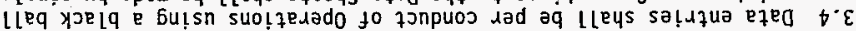

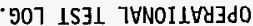

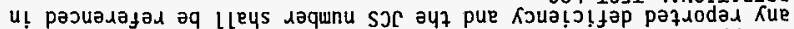

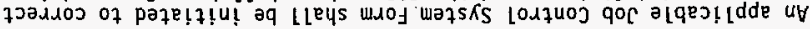

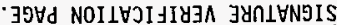

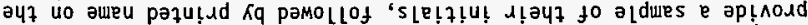
LLeys aunpasoid s!47 u! sdofs pafalduos tfo 6u! Le!f!u! suaslad LLV

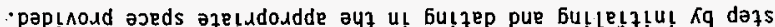

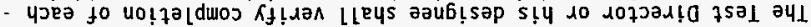

\section{say00zy $0^{\prime} \varepsilon$}

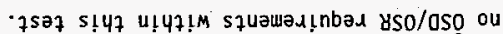

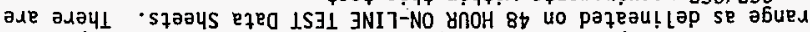
pəzวadxa әч7 to \%0I U!47!M әq 7 snu 'e!

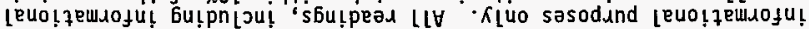

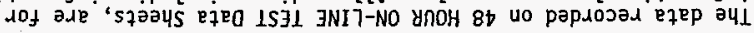

SNOILNOJYd ONV SLIWII 6'Z 
4.1 The following supplies may be needed to perform this procedure:

- Chevron Machine 0i1 ISO AW 150 (4.5 gallons minimum)

- Hazardous Material Spill Kit for liquids

- Hearing Protection

- Eye Protection

- Gloves

- Cellulose Sponges

- Cl amp-On amp meter

- Tools required for disassembly and reassembly

- Stop Watch

- Flashight

- EG Heat Transfer Fluid 100 (55 gallons) (NOTE: Only if the system has been drained)

4.2 The following procedures will be needed to perform this procedure:

- Calibration Procedures 6-TF-508 and 6-TF-514

4.3 The following conditions must be met before this procedure may be performed:

4.3.1 A pre-job safety meeting shall be conducted prior to performing this Operational Test Procedure.

Test Director Pauleculle Date $310-97$

NOTE - The following Checklists and valving may be performed in any order.

4.3.2 ENSURE the position of valves as required in the following checklists:

- CHECKLIST 1 - COOLING WATER VALVING

- CHECKLIST 2 - PROCESS AIR VALVING

- CHECKLIST 3 - INSTRUMENT AIR VALVIÑG

- CHECKLIST 4 - ACCUMULATOR INSTRUMENT AIR VALVING

4.3.3 Ensure work permit for energized system(s) is in effect for testing.

4.3.4 ENSURE the position of disconnects and breakers as required in the following checklist:

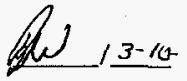

- CHECKLIST 5 - ELECTRICAL LINE-UP 


\subsection{PREREQUISITES}

Initials/Date

4.1 The following supplies may be needed to perform this procedure:

- Chevron Machine 0il ISO AW 150 (4.5 gallons minimum)

- Hazardous Material Spill Kit for liquids

- Hearing Protection

- Eye Protection

- Gloves

- Cellulose Sponges

- Clamp-On amp meter

- rools required for disassembly and reassembly

- Stop Watch

- Flashlight

- EG Heat Transfer Fluid 100 (55 gallons) (NOTE: Only if the system has been drained)

4.2 The following procedures will be needed to perform this procedure:

- Calibration Procedures 6-TF-508 and 6-TF-514

4.3 The following conditions must be met before this procedure may be performed:

4.3.1 A pre-job safety meeting shall be conducted prior to performing this Operational Test Procedure.

Test Director Pauldoullo D_ Date 3-17-97

NOTE - The following Checklists and valving may be performed in any order.

4.3.2 ENSURE the position of valves as required in the following checklists:

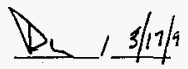

- CHECKLIST 1 - COOLINg WATER VALVING

- CHECKLIST 2 - PROCESS AIR VALVING

- CHECKLIST 3 - INSTRUMENT AIR VALVING

- CHECKLIST 4 - ACCUMULATOR INSTRUMENT AIR VALVING

4.3.3 Ensure work permit for energized system(s) is in effect for testing.

4.3.4 ENSURE the position of disconnects and breakers as required in the following checklist:

Q) $p / 3 / 17 \%$

- CHECKLIST 5 - ELECTRICAL LINE-UP 


\subsection{PREREQUISITES (Cont.)}

Initials/Date

NOTE - Surge Tank site glass does not have markings therefore, approximation is acceptable.

4.3.5 Verify that the glycol/water level appears above halfway, and below three quarters, of the Surge Tank site glass and Add glycol/water (50/50 mixture) if required.

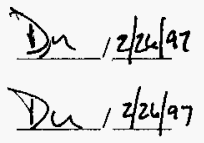

4.3.6 Verify that oil level is above mark and ADD, if required.

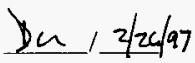

4.3.8 Drain the compressor intake and receiver tank of any accumulated moisture.

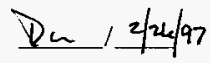

4.3.9 Verify the following work packages have been performed in support of this OTP:

$2 \mathrm{E}-96-1077,2 \mathrm{E}-96-368,2 \mathrm{E}-95-748$

E. W. Whis

QC $\sum n$ W

Date $2-2 y-57$

NOTE - Verification shall be done before the step associated with that instrument.

4.3.10 Verify the current calibration, and record the calibration date and calibration due date on the table below.

\begin{tabular}{|lr|r|r|}
\hline & INSTRUMENT & CALIBRATION & CALIBRATION \\
BATE & DUE DATE \\
\hline SA-PSL-801 & (CMP-801 LO LUBE OIL TRIP) & $1-14-97$ & $1-14-98$ \\
\hline SA-PSL-802 & (CMP-802 LO LUBE OIL TRIP) & $1-14-97$ & $1-14-98$ \\
\hline SA-PSH-801 & (HI INTER STG PRESS IRIP) & $2-19-97$ & $2-19-98$ \\
\hline SA-PSH-802 & (HI INTER STG PRESS TRIP) & $2-19-97$ & $2-19-98$ \\
\hline SA-PSH-803 & (CMP-801 DISCH PRESS TRIP) & $2-18-97$ & $2-18-98$ \\
\hline SA-PSH-804 & (CMP-802 DISCH PRESS TRIP) & $2-18-97$ & $2-18-98$ \\
\hline IA-PSH-810A & (IA-PCV-803A DISCH PRESS HI) & $2-20-97$ & $2-20-98$ \\
\hline IA-PSH-810B & (IA-PCV-803B DISCH PRESS HI) & $2-20-97$ & $2-20-98$ \\
\hline IA-PISL-801 & (SA-PISL-801 SA HDR-6 PRESS ALARM) & $2-20-97$ & $2-20-98$ \\
\hline
\end{tabular}

\begin{tabular}{|c|c|c|}
\hline 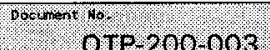 & Wom.d. & (3) \\
\hline
\end{tabular}


HNF-SD-WM-OTR-231, Rev 0

Page 36 of 165

SLA

\subsection{PREREQUISITES (Cont.)}

Initials/Date

NOTE - Surge Tank site glass does not have markings therefore, approximation is acceptable.

4.3.5 Verify that the glycol/water level appears above halfway, and below three quarters, of the Surge Tank site glass and Add glycol/water (50/50 mixture) if required.

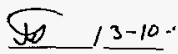

4.3.6 Verify that oil level is above mark and ADD, if required.

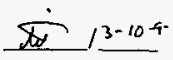

4.3.7 Verify the exterior surface and surrounding area of the entire system are free from dust or debris, and clean if necessary.

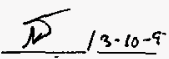

4.3.8 Drain the compressor intake and receiver tank of any accumulated moisture.

to $1>-10-9$

4.3.9 Verify the following work packages have been performed in support of this OTP:

2E-96-1077, 2E-96-368, 2E-95-748

QC Eタ. Wyane Date $3-10-57$

NOTE - Verification shall be done before the step associated with that instrument.

4.3.10 Verify the current calibration, and record the calibration date and calibration due date on the table below.

pos $13+10^{-9}$

\begin{tabular}{|c|c|c|}
\hline INSTRUMENT & $\begin{array}{l}\text { CALIBRATION } \\
\text { DATE }\end{array}$ & $\begin{array}{l}\text { CALIBRATION } \\
\text { DUE DATE }\end{array}$ \\
\hline SA-PSL-801 $\quad$ (CMP-801 LO LUBE 0IL TRIP) & & \\
\hline SA-PSL-802 (CMP-802 LO LUBE OIL TRIP) & & \\
\hline SA-PSH-801 (HI INTER STG PRESS TRIP) & 0 & \\
\hline SA-PSH-802 (HI INTER STG PRESS TRIP) & $Q^{x} x^{1}$ & \\
\hline 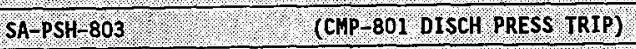 & & \\
\hline SA-PSH-804 (CMP-802 DISCH PRESS TRIP) & & \\
\hline (IA-PCY-803A DISCH PRESS HI) & & \\
\hline (IA-PCV-803B DISCH PRESS HI) & & \\
\hline IA-PISL-801 (SA-PISL-801 SA HDR-6 PRESS ALARM) & & \\
\hline
\end{tabular}




\subsection{PREREQUISITES (Cont.)}

NOTE - Surge Tank site glass does not have markings therefore, approximation is acceptable.

4.3.5 Verify that the glycol/water level appears above halfway, and below three quarters, of the Surge Tank site glass and Add glycol/water (50/50 mixture) if required.

4.3.6 Verify that oil level is above mark and ADD, if required.

Q. $13 \ln 197$

$D \leq / s|h| 9$

4.3.7 Verify the exterior surface and surrounding area of the entire system are free from dust or debris, and clean if necessary.

$D=|3| 17 \mid \mathrm{r} ;$

4.3.8 Drain the compressor intake and receiver tank of any accumulated moisture.

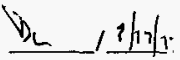

4.3.9 Verify the following work packages have been performed in support of this OTP:

2E-96-1077, 2E-96-368, 2E-95-748 Opeviously verified by Frnie QC PQWll.Wemer Date 3-17-97

NOTE - Verification shal1 be done before the step associated with that instrument.

4.3.10 Verify the current calibration, and record the calibration date and calibration due date on the table below.

Do $13 / 17 / 5$

\begin{tabular}{|c|c|c|}
\hline INSTRUMENT & $\begin{array}{l}\text { CALIBRATION } \\
\text { DATE }\end{array}$ & $\begin{array}{l}\text { CALIBRATION } \\
\text { DUE DATE }\end{array}$ \\
\hline (CMP-801 LO LUBE OIL TRIP) & & \\
\hline (CMP-802 LO LUBE 01L TRIP) & & \\
\hline (HI INTER STG PRESS TRIP) & & \\
\hline (HI INTER STG PRESS TRIP) & & \\
\hline (CMP-8O1 DISCH PRESS TRIP) & & \\
\hline (CMP-802 DISCH PRESS TRIP) & & \\
\hline (IA-PCV $-803 \mathrm{~A}$ DISCH PRESS HI) & & \\
\hline (1A-PCV-803B DISCH PRESS HI) & & \\
\hline IA-PISL-80I (SA-PISL-801 SA HDR-6 PRESS ALARM) & & \\
\hline
\end{tabular}




\subsection{PREREQUISITES (Cont.)}

Initials/Date

4.3.11 All Operators, Shift Managers, and other personnel performing this Procedure who will be initialing and signing Procedure shall enter their printed name, signature and initials on the PROCEDURE PERFORMER SIGNATURE SHEET.

PDA / 310:

4.3.12 Notify the Tank Farm Shift Manager that the Operation Test Procedure is to be performed.

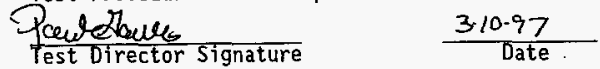

Section 4.0 PREREQUISITES is complete.

4.3.13 Test Director VERIFY that section 4.0 has been COHPLETED by SIGNING below.

$\frac{\text { tacelofoceo }}{\text { Test Director signature }} \frac{3-10.97}{\text { Date }}$

4.3.14 Quality Control Inspector VERIFY that section 4.0 has been COMPLETED by SIGNING beTow.

En- Werex Quality Contyol Inspector Signature

$\frac{3-10-52}{\text { Date }}$

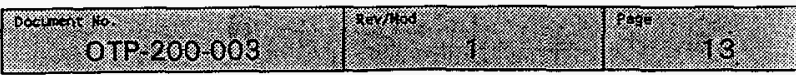




\subsection{PREREQUISITES (Cont.)}

Initials/Date

4.3.11 All Operators, Shift Managers, and other personnel performing this Procedure who will be initialing and signing Procedure shall enter their printed name, signature and initials on the PROCEDURE PERFORMER SIGNATURE SHEET.

PQL, 3-17

4.3.12 Notify the Tank Farm Shift Manager that the Operation Test Procedure is to be performed. Fauldaullo Test Director Signature

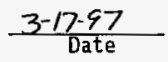

Section 4.0 PREREQUISITES is complete.

4.3.13 Test Director VERIFY that section 4.0 has been COMPLETED by SIGNING below.

toufthacllo

Test Director Signature

$\frac{3-17-97}{\text { Date }}$

4.3.14 Quality Control Inspector VERIFY that section 4.0 has been COMPLETED by SIGNING below.

Canti. Werner Quality Control Inspector signature

$\frac{3-17-9 ?}{\text { Date }}$

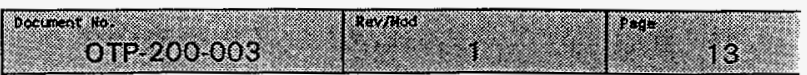




\subsection{PROCEDURE}

Initials/Date

NOTE - Interruption of data collection, unless noted otherwise, will be at the discretion of the Test Director.

\subsection{PERFORM 48 HOUR ON-LINE TEST}

NOTE - This section will be used for both compressors, SA-CMP-801 and SA-CMP-802.

- Each compressor shall be run separately for 48 hours.

5.1.1 VERIFY position of the glycol pump control switch, SW-1, on Panel GLY-PNL-801 Glycol Control Panel is OFF.

5.1.2 VERIFY position of the compressor bypass switch, SW-3, on Panel GLY-PNL-801 Glycol Control Panel is OFF.

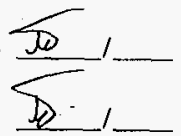

5.1.3 POSITION cooling fan contral switch, SS-1, to AUTO on Panel GLY-PNL-801 Glycol Control Panel.

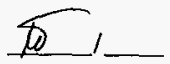

5.1.4 CLOSE breaker EDS-CKT-844 in compartment G1-R at EDS-MCC-803, energizing the feeder circuit to the GLY-PNL-801 Glycol Control Panel.

Res $13 / 101$

5.1.5 POSITION disconnect switch, GLY-DS-801 GLYCOL PUMP \#1 Disconnect, to ON, for Glycol Pump \#1 (GLY-P-801).

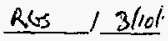

5.1.6 POSITION disconnect switch, GLY-DS-802 GLYCOL PUMP \#2 Dísconnect, to ON, for G7ycol Pump \#2 (GLY-P-802).

RGS $/ 3 / 10 k$

5.1.7 POSITION disconnect switch, GLY-DS-803 GLYCOL FAN BANK \#1 DISCONNECT, to ON for Glycol Fan Bank \#1.

5.1.8 POSITION disconnect switch "GLY-DS-804 GLYCOL FAN BANK \#2 DISCONNECT, to ON for Glycol Fan Bank \#2.

RGs / $3 / 1 / 1 /$ RGS $/ 3 / / 16$

5.1.9 POSITION breaker \#21 for SA-PNL-806 (LOCAL PANEL ALARM), breaker \#22 for Panel IA-PNL-805 (IA DRYER CONTROL PANEL) and breaker $\$ 24$ (bleeder solenoid) on distribution pane1 EDS-DP-809 241-A-271 BLDG DIST PANEL, to ON.

Ros $13 / 101$

5.1.10 SET selector switch, SS-1, on SA-PNL-803 SA CMP 801 \& 802 SEQUENCER PNL, to SA-CMP-801 (Tead unit)". 


\subsection{PROCEDURE}

NOTE - Interruption of data collection, unless noted otherwise, will be at the discretion of the Test Director.

\subsection{PERFORM 48 HOUR ON-LINE IEST}

NOTE - This section will be used for both compressors, SA-CMP-801 and SA-CMP-802.

- Each compressor shall be run separately for 48 hours.

5.1.1 VERIFY position of the glycol pump control switch, SW-1, on Panel GLY-PNL-801 Glycol Control Panel is OfF.

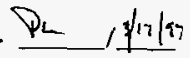

5.1.2 VERIFY position of the compressor bypass switch, SW-3, on Panel GLY-PNL-801 Glycol Control Panel is OFF.

5.1.3 POSITION cooling fan control switch, SS-1, to AUTO on Panel GLY-PNL-801 Glycol Control Panel.
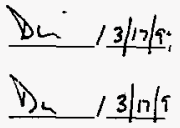

5.1.4 CLOSE breaker EDS-CKT-844 in compartment GI-R at EDS-MCC-803, energizing the feeder circuit to the GLY-PNL-801 GIycol Control Pane?.

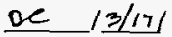

5.1.5 POSITION disconnect switch, GLY-DS-801 GLYCOL PUMP \# 1 Disconnect, to ON, for Glycol Pump \#1 (GLY-P-801).

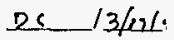

5.1.6 POSITION disconnect switch, GLY-DS-802 GLYCOL PUMP \#2 Disconnect, to ON, for Glycol Pump \#2 (GLY-P-802).

5.1.7 POSITION disconnect switch, GLY-DS-803 GLYCOL FAN BANK \#1 DISCONNECT, to ON for Glycol Fan Bank \#1.
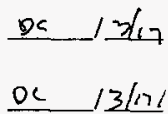

5.1.8 POSITION disconnect switch "GLY-DS-804 GLYCOL FAN BANK \#2 DISCONNECT, to ON for Glycol Fan Bank $\# 2$.

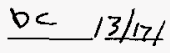

5.1 .9 POSITION breaker \#21 for SA-PNL-806 (LOCAL PANEL ALARM), breaker \#22 for Pane1 IA-PNL-805 (IA DRYER CONTROL PANEL) and breaker \#24 (bleeder solenoid) on distribution panel EDS-DP-809 241-A-271 BLDG DIST PANEL, to ON.

DC $13 / 17 /$

5.1.10 SET selector switch, SS-1, on SA-PNL-803 SA CMP 801 \& 802 SEQUENCER PNL, to SA-CMP-801 (lead unit)".

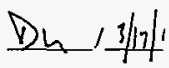




\subsection{PERFORM 48 HOUR ON-LINE TEST (Cont.)}

Initials/Date

5.1.11 CLOSE breaker EDS-CKT-831 in compartment C3 of EDS-MCC-803 for SA-CMP-801.

Res $/ 3 / 10 h$

5.1.12 CLOSE breaker EDS-CKT-837 in compartment E3 of EDS-MCC-803 for SA-CMP-802.

Rers $/ 3 / 10 / 7$

5.1.13 TURN Power Switch located on IA-PNL-805 (IA DRYER CONTROL PANEL) to ON.

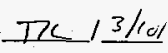

5.1.14 VERIFY the IA-PNL-805 (IA DRYER CONTROL PANEL) lights are illuminated.

$\pi / 3 / 10$

5.1.15 SET the dryer Cycle selector switch, located on IA-PNL-805, to 10 min fix cycle.

$T+13 \%$

5.1.16 VERIFY the "STOPPED" 1ight on the SA-PNL-801 is il luminated.

Is $\mid 3 /$ ide

Th $/ 3 / 10 / 4$

5.1.17 RESET shutdown alarm.

5.1.18 VERIFY no shut down indicators are illuminated on the SA-PNL-801; with the exception of the lube oil low pressure and cooling fluid low flow indicators.

$x \quad(3 / 10 / \%$

5.1.19 VERIFY Manual/Auto switch, SS-1 on SA-PNL-801 and on SA-PNL-802 are both in the AUTO position.

TC $/ 3 / \mathrm{sal}$

5.1.20 VERIFY/POSITION the valves as shown in the table below. $13 / \mathrm{d} / \mathrm{s}$

\begin{tabular}{|c|c|c|c|c|c|}
\hline VALVE & POSITION & INIT & VALYE & POSITION & INIT \\
\hline $\begin{array}{l}S A-V-825 \text { (PA-701-A-8) } \\
\text { ACCUMULATOR INLET ISO }\end{array}$ & CLOSEO & $\pi^{C}$ & $\begin{array}{l}S A-V=859(\mathrm{PA}-701 \mathrm{~A}-46) \\
\mathrm{CMP} 801 \text { (OAD CONT AIR ISO }\end{array}$ & OPEN & \\
\hline $\begin{array}{l}\text { SA-V - } 827 \quad(P A-701-A-9) \\
\text { ACCUMULATOR BYP VLY }\end{array}$ & OPEN & $\pi$ & $\begin{array}{l}\mathrm{SA}-8.858(\mathrm{PA}-701-\mathrm{A}-47) \\
\mathrm{CMP} 802 \mathrm{OAD} \text { CONT AIR ISO }\end{array}$ & OPEN & \\
\hline $\begin{array}{l}\text { SA V } 826 \text { (PA-701-A- } 10) \\
\text { ACCUMULATOR OUTLET I SO }\end{array}$ & CLOSED & & $\begin{array}{l}\mathrm{GLY} V-836,(\mathrm{CH}-701-\mathrm{A}-20) \\
\mathrm{GLY}-\mathrm{SOV}-830 \mathrm{BYP}\end{array}$ & $0 \mathrm{p}$ & \\
\hline $\begin{array}{l}\text { SA-V-B64 }(P A-701-A-39) \\
\text { SA CHP UNLDR AIR } \\
\text { SUPPLY }\end{array}$ & OPEN & & & & \\
\hline
\end{tabular}

5.1.21 PUSH "START" buttons on SA-PNL-801 and SA-PNL-802 and VERIFY that the "AUTO READY" lights are illuminated.

$\sqrt{x} / 3 / 0$

$6.1,211$ OPEN UALue SA-V-925 seerexception stext 


\subsection{PERFORM 48 HOUR ON-LINE TEST (Cont.)}

5.1.11 CLOSE breaker EDS-CKT-831 in compartment C3 of EDS-MCC-803 for SA-CMP-801.

De 12 ho

5.1.12 CLOSE breaker EDS-CKT-837 in compartment E3 of EDS-MCC-803 for SA-CMP-802.

DC $/ 3 / 14 / 9$

5.1.13 TURN Power Switch located on IA-PNL-805 (IA DRYER CONTROL PANEL) to ON.

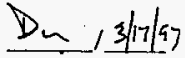

5.1.14 VERIFY the IA-PNL-805 (IA DRYER CONTROL PANEL) lights are illuminated.

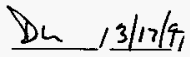

5.1.15 SET the dryer Cycle selector switch, located on IA-PNL-805, to $10 \mathrm{~min}$ fix cycle.

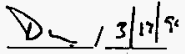

5.1.16 VERIFY the "STOPPED" 1 ight on the SA-PNL-801 is illuminated.

5.1.17 RESET shutdown alarm.

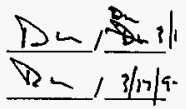

5.1.18 VERIFY no shut down indicators are illuminated on the SA-PNL-801; with the exception of the lube oil low pressure and cooling fluid low flow indicators.

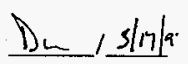

5.1.19 VERIFY Manual/Auto switch, SS-1 on SA-PNL-801 and on SA-PNL-802 are both in the AUTO position.

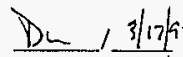

5.1.20 VERIFY/POSITION the valves as shown in the table below. $D_{L} / 3 / 17 / 5$;

\begin{tabular}{|c|c|c|c|c|c|}
\hline VALVE & POSIIION & INIT & VALVE & POSITION & INIT \\
\hline $\begin{array}{l}\text { SA-V-825 (PA-701-A-8) } \\
\text { ACCUMULATOR INLET ISO }\end{array}$ & ftosevis & w & $\begin{array}{l}\text { SA-Y } 859 \text { (PA-701-A-46) } \\
\text { CMP } 801 \text { IOAD CONT AIR ISO }\end{array}$ & OPEN & $D_{n}$ \\
\hline $\begin{array}{l}\text { SA-V-827 }(P A-701-A-9) \\
\text { ACCUMULATOR BYP VLV }\end{array}$ & OPEN & $D$ & $\begin{array}{l}\text { SA-V-858 (PA-701-A - } 47) \\
\text { CHP } 802 \text { LOAD CONT AIR ISO }\end{array}$ & OPEN & $\nabla_{L}$ \\
\hline $\begin{array}{l}\text { SA-Y-826 (PA-701-A-10) } \\
\text { ACCUMULATOR OUTLET ISO }\end{array}$ & CLOSEO & & $\begin{array}{l}\mathrm{GLY} V=836 \quad(C W-701-A-20) \\
G L Y-S O V-830 \text { BYP }\end{array}$ & OPEN & $D_{n}$ \\
\hline $\begin{array}{l}\text { SA V - } 864 \quad(P A-701-A-39) \\
\text { SA CMP UNLDR AIR } \\
\text { SUPPLY }\end{array}$ & OPEN & & & & \\
\hline
\end{tabular}

5.1.21 PUSH "START" buttons on SA-PNL-801 and SA-PNL-802 and VERIFY that the "AUTO READY" lights are illuminated.

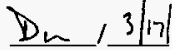




\subsection{PERFORM 48 HOUR ON-LINE TEST (Cont.)}

Initials/Date

5.1.22 TURN glycol pump control switch, SW-1, on Panel GLY-PNL-801 Glycol Control Panel to ON.

5.1.23 VERIFY Glycol pump GLY-P-801 or GLY-P-802 starts.

NOTE - Time will be measured using stopwatch.
$+3 / 30 / m$

pe $/ 3 / \mathrm{s} / \mathrm{s}_{\mathrm{s}}$

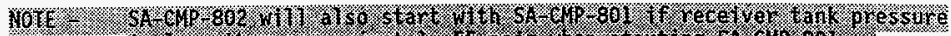

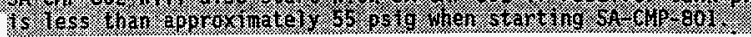

\subsubsection{VERIFY compressor SA-CMP-801 starts after 5 to 20 seconds}

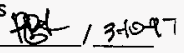

RECORD operating Pump number (GLY-P-801 or GLY-P-802)

$802 / 3 / 4 / 9$

RECORD time Tag between the pump and compressor

$6 \sec / 3 / 19 / 9$.

RECORD Pump Suction Pressure GLY-PI-801 (PI-701-A-17)

$1 / 3 / 40 / 9$ RECORD PÜm Díscharge Pressure GLY-PI-803 (PI-701-A-16)

$55 / 3 / 1 / 4$

5.1.25 VERIFY SA-CMP-801 and SA-CMP-802 running lights are

illuminated AND WAIT unti1 SA G M

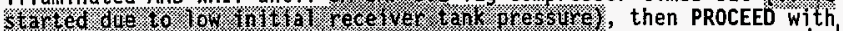
procedure. TKK $\mid$ 3livls

NOTE - After positioning the valves in the following table the Shift Manager should be notified that the $A, A X, A Y$ and $A Z$ Tank Farms are being supplied air by the new 701-A compressed air system.

5.1.26 POSITION the valves as shown in the table below.

$\pi / 1 / 4 / 9$

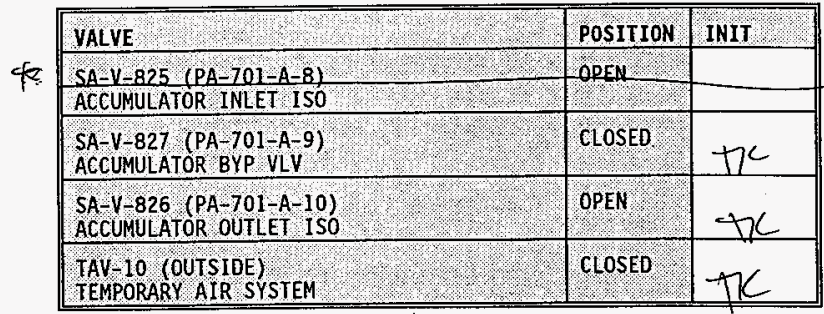

5.1.27 ALLOW the receiver pressure to increase and VERIFY that SA-CMP-801 unloads.

$+x, 3 / 7$,

- See excrato A1

陪 3-10.97 


\subsection{PERFORM 48 HOUR ON-LINE TEST (Cont.)}

5.1.22 TURN glycol pump control switch, SW-1, on Pane] GLY-PNL-801 Glycol Control Panel to ON.

5.1.23 VERIFY G1ycol PUmp GLY-P-801 or GLY-P-802 starts.

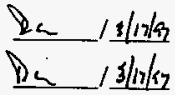

NOTE - Time will be measured using stopwatch.

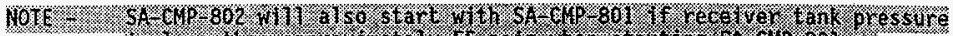

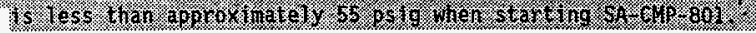

5.1.24 VERIFY compressor SA-CMP-801 starts after 5 to 20 seconds

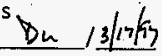

RECORD operating Pump number (GLY-P-801 or GLY-P-802)

REcon

RECORD time Tag between the pump and compressor

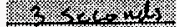

RECOORD Pump Süction Pressure GLY-PI-801 (PI-701-A-17)

(3)

RECORD PumP Díscharge Pressure GLY-PI-803 (PI-701-A-16) (1)

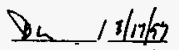

$D_{n} / 3 / 12 / 59$

D) $13 / 17 / 57$

D.

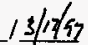

5.1.25 VERIFY SA-CMP-801 and SA-CMP-802 running lights are

illuminated AND WAIT unti1 SACGMP 802 7ag compressor times out /f If

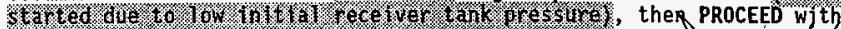
procedure.

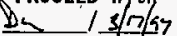

NOTE - After positioning the valves in the following table the Shift Manager should be notified that the $A, A X, A Y$ and $A Z$ Tank Farms are being supplied air by the new 701-A compressed air system.

5.1.26 POSITION the valves as shown in the table below.

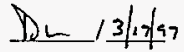

\begin{tabular}{|c|c|c|}
\hline VALVE & POSITION & INIT \\
\hline $\begin{array}{l}\text { SA V - } 825 \text { (PA-701-A-8) } \\
\text { ACCUMULATOR INLET ISO }\end{array}$ & OPEN & \\
\hline $\begin{array}{l}\text { SA-V-827 (PA-701-A-9) } \\
\text { ACCUMULATOR BYP VLV }\end{array}$ & CLOSED & \\
\hline $\begin{array}{l}\text { SA-V -826 (PA-701-A-10) } \\
\text { ACCUMULATOR OUTLET ISO }\end{array}$ & OPEN & \\
\hline $\begin{array}{l}\text { TAV } 10 \text { (OUTSIDE) } \\
\text { TEMPORARY AIR SYSTEM }\end{array}$ & CLOSEO & \\
\hline
\end{tabular}

5.1.27 ALLOW the receiver pressure to increase and VERIFY that SA-CMP-801 unloads.

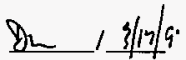




\subsection{PERFORM 48 HOUR ON-LINE TEST (Cont.)}

RECORD maximum and minimum receiver pressure below, as read from IA-PISL-801.

SA-CMP-801 unloads at 100 psig

SA-CMP-801 loads at 94 psig

5.1.29 IF the receiver pressure is not within 97-103 $(100 \pm 3)$ psig, THEN ADJUST the free air regulator per INGERSOLL-RAND COMPRESSOR PHE MAINTENANCE INSTRUCTIONS section 7 , until the desired receiver pressure of 100 psig is achieved.

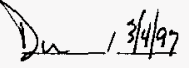

5.1.30 RECORD motor currents in compartment C3 of EDS-MCC-803 fo SA-CMP-801 below:

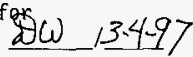

(A) No Load 62 , Amps/Fu11 Load 80 Amps

(B) No Load 70 Amps/Ful1 Load 84 Amps

(C) No Load_ 64 Amps/Ful1 Load 82 Amps

5.1.31 OPEN Valve IA-Y-826, IA DRY OUTLET ISO, then SLOWLY OPEN valve IA-V-809, IA DRY INLET ISO to allow air to flow into the air dryer.

5.1.32 PRESS reset button located on IA-PNL-805.

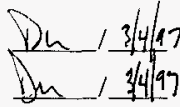

5.1.33 VERIFY either the left $\mathrm{OR}$ right tower indicating light is ilTuminated on dryer Sensatherm controller.

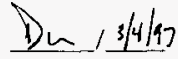
Left Right

5.1.34 CLOSE the dryer bypass valve IA-V-820, IA DRY BYPASS VALVE.

5.1.35 VERIFY purge rate pressure setting is set at 45 psig.

5.1.36 IF adjustment is required, REGULATE flow with valve IA-V-825 to 45 psi.
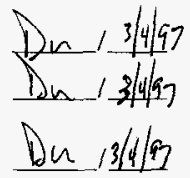

NOTE - Dew point initial color should be yellow, but should turn green.

5.1.37 WHEN dew point color changes to green, SELECT $-40^{\circ} \mathrm{F}$.

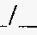


5.1 PERFORM 48 HOUR ON-LINE TEST (Cont.)

5.1.28 RECORD maximum and minimum receiver pressure below, as read from $Y A-P I S L-801$.

SA-CMP-801 untoads at 501 psig

SA-CMP-801 loads at 95 psig

5.1.29 If the receiver pressure is not within 97-103 (100 \pm 3$)$ psig, THEN ADJUST the free air regulator per INGERSOLL-RAND COMPRESSOR PHE MAINTENANCE INSTRUCTIONS section 7 , until the desired receiver pressure of 100 psig is achieved.

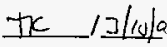

5.1.30 RECORD motor currents in compartment C3 of EDS-MCC-803 for SA-CMP-801 below:
(A) No Load Amps/Ful1 Load Amps
(B) No Load Aimps/Full Load Amps
(C) No Load Amps/Fuil Load Anips

5.1.31 OPEN Valve IA- $V-826$, IA DRY OUTLET ISO, then SLOWLY OPEN valve IA-V-809, IA DRY INLET ISO to allow air to flow into the air dryer.

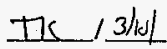

5.1.32 PRESS reset button located on IA-PNL-805. TK $13 / 1 \%$

5.1.33 VERIFY either the left OR right tower indicating light is illuminated on dryer Sensatherm controller.

$5 \pi / 3 \%$ Left Right

5.1.34 CLOSE the dryer bypass valve IA-V-820, IA DRY BYPASS VALVE:

ThC $/ 3 / \mathrm{s}$

5.1.35 VERIFY purge rate pressure setting is set at 45 psig. The $13 \%$

5.1.36 If adjustment is required, REGULATE flow with valve IA-V-825 to 45 psi.

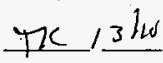

NOTE - Dew point initial color should be yellow, but should turn green.

5.1.37 WHEN dew point color changes to green, SELECT $-40^{\circ} \mathrm{F}$. 


\subsection{PERFORM 48 HOUR ON-LINE TEST (Cont.)}

Initials/Date

5.1 .28

RECORD maximum and minimum receiver pressure below, as read from IA-PISL-801.

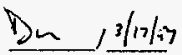

SA-CMP-801 unloads at 100 psig

SA-CMP-801 loads at 75 psig

5.1.29 IF the receiver pressure is not within 97-103 (100 \pm 3$)$ psig, THEN ADJUST the free air regulator per INGERSOLL-RAND COMPRESSOR PHE MAINTENANCE INSTRUCTIONS section 7 , until the desired receiver pressure of 100 psig is achieved.

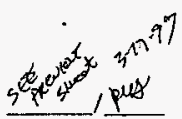

5.1 .30

RECORD motor currents in compartment C3 of EDS-MCC-803 SA-CMP-801 below:

(A) No Load con O Amps/Ful1 Lead Amps

(B) No Load Amps/Full Load Amps

(C) No Load Amps/Full Load Amps

5.1.31 OPEN valve IA-V-826, IA DRY OUTLET ISO, then SLOWLY OPEN valve IA-V-809, IA DRY INLET ISO to allow air to flow into the air dryer.

5.1.32 PRESS reset button located on IA-PNL-805.

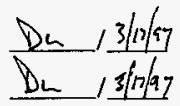

5.1.33 VERIFY either the left $\underline{O R}$ right tower indicating light is $i l l u m i n a t e d$ on dryer Sensatherm controller.

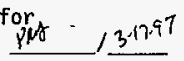
Left Regenentitin Right DR ping

5.1.34 CLOSE the dryer bypass valve IA-V-820, IA DRY BYPASS VALVE.

5.1 .35

VERIFY purge rate pressure setting is set at 45 psig.

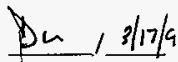

5.1 .36

If adjustment is required, REGULATE flow with valve IA-V-825 to $45 \mathrm{psi}$.

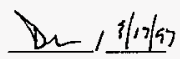

Dew point initial color should be yellow, but should

NOTE turn green.

5.1.37 WHEN dew point color changes to green, SELECT $-40^{\circ} \mathrm{F}$.

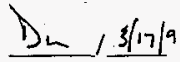


NOTE - The 48-hour on-1ine test shall not start until each tower has cycled twice. Two cycles take about 20 minutes.

5.1 .38 REPOSITION the following valves to allow air to flow from the new CAS to the tank farms.

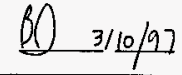

\begin{tabular}{|c|c|c|c|c|c|}
\hline .1 .4 & POSITION & INIT & 4.0 .0$. & rosirION & INIT \\
\hline $\begin{array}{l}\text { SA-V - } 841 \text { (PA- 701-A-18) } \\
\text { SA AUX SUPPLY DRN VLV }\end{array}$ & (1) $8 *$ & BD & $1 A-702-20$ & A-B & 30 \\
\hline $\mathrm{IA}-702-21$ & A.B & 20 & $1 A-702-22$ & A. B & e \\
\hline
\end{tabular}

* Three-way valves, normal flow is perpendicular to pipe run.

NOTE - The interim compressor will remain running for the first two hours OR until the TD is confident that the new compressors and equipment is functioning as designed.

\begin{tabular}{|c|c|c|c|c|c|}
\hline VALVE & POSITION & INIT & VALVE & POSITION & IHF \\
\hline $\begin{array}{l}\text { IA-V }-857 \text { (IA-702-10) } \\
\text { IA-IK } 802 \text { OUTLET ISO VLV }\end{array}$ & OPEN & 3 & $\begin{array}{l}\text { SA-V-834 (PA-701-A-13) } \\
\text { SA TO A FARM ISO }\end{array}$ & OPEN & \\
\hline $\begin{array}{l}\text { IA-V-838 (IA-701-A-37) } \\
\text { IA TO } 241 \text { A } 150 \text {. }\end{array}$ & OPEN & & $\begin{array}{l}\text { SA-V-835 (PA-701-A-14) } \\
\text { SA-TO AX, AY, AZ FARH ISO }\end{array}$ & OPEN & $y$ \\
\hline $\begin{array}{l}\text { IA-V-837 (IA-701-A-38) } \\
\text { IA TO AY AZ FARM ISO }\end{array}$ & OPEN & & & & \\
\hline
\end{tabular}

5.1.40 MONITOR AND RECORD the required items on the Data Sheet at 15 minute intervais for the first hour, or until the system is stabilized, then continue readings at hourly intervals for the remainder of the first 8 hours.

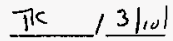

5.1.41 WHEN Test Director is assured of Compressor operation, CLOSE valves TAV -4 or TAV- 5 .

5.1.41.1 POSITION I-0 (Start-Stop) button on air dryer control panel TAS-1 to "0" position, VERIFY power 7 amp is NOT 111 uminated.

5.1.41.2 SHUTDOWN/STOP interim compressor as per instructions posted on panel door.

5.1.42 If any unusual conditions are noticed, RECORD the details on an OTP Exception Log sheet AND NOTIFY the Test Director and Systems Cog Engineer. 
5.1 PERFORM 48 HOUR ON-LINE TEST (Cont.)

NOTE - The 48-hour on- 7 ine test shall not start until each tower has cycled twice. Two cycles take about 20 minutes.

5.1.38 REPOSITION the following valves to allow air to flow from the new CAS to the tank farms.

DL $/ \sin \mid s$

\begin{tabular}{|c|c|c|c|c|c|}
\hline VALVE & POSITION & INIT & VALVE & POSITION & ING \\
\hline $\begin{array}{l}\text { SA-V } 841,(P A-701-A-18) \\
\text { SA AUX SUPPLY DRN VLV }\end{array}$ & $6^{-6 *}$ & 8 & $1 A-702-20$ & $A^{-B}$ & 18 \\
\hline $\mathrm{IA}-702-21$ & A-B & 0 & $1 \mathrm{~A}-7,02-22$ & $A-B$ & is \\
\hline
\end{tabular}

* Three-way valves, normal flow is perpendicular to pipe run.

NOTE - The interim compressor will remain running for the first two hours OR unti] the TD is confident that the new compressors and equipment is functioning as designed.

5.1.39 POSITION the following valves to allow air to flow to the A-complex Tank Farms.

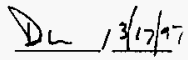

\begin{tabular}{|c|c|c|c|c|c|}
\hline VALVE & POSITION & INIT & VALVE & POSITION & INIT \\
\hline $\begin{array}{l}\text { IA-V-857 (IA-702-10) } \\
\text { IA-TK-802 OUTLET ISO VLV }\end{array}$ & OPEN & & $\begin{array}{l}\text { SA-V-834 (PA-701-A-13) } \\
\text { SA TO A FARM ISO }\end{array}$ & OPEN & $k<$ \\
\hline $\begin{array}{l}\text { IA }- \text { - }-838 \text { (IA-701-A-37) } \\
\text { IA IO } 241 \text { A ISO }\end{array}$ & OPEN & & $\begin{array}{l}\text { SA-V-835, }(\mathrm{PA}-701-\mathrm{A}, 14) \\
\mathrm{SA}-\mathrm{TO} \mathrm{AX}, \mathrm{AY}, \mathrm{AZ} \text { FARM I SO. }\end{array}$ & OPEN & \\
\hline $\begin{array}{l}\text { IA-V-837 (IA-701-A-38) } \\
\text { IA TO AY AZ FARM ISO }\end{array}$ & OPEN & & & & \\
\hline
\end{tabular}

5.1.40 MONITOR AND RECORD the required items on the Data Sheet at 15 minute intervals for the first hour, or until the system is stabilized, then continue readings at hourly intervals for the remainder of the first 8 hours.

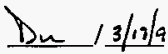

5.1.41 WHEN Test Director is assured of Compressor operation, CLOSE valves TAV -4 or TAV -5 .

5.1.41.I POSITION I-0 (Start-Stop) button on air dryer control pane1 TAS-1 to " 0 " position, VERIFY power lamp is NOT 111 uminated.

5.1.41.2 SHUTDOWN/STOP interim compressor as per instructions posted on panel door.

5.1.42 IF any unusual conditions are noticed, RECORD the details on an OTP Exception Log sheet AND NOTIFY the Test Director and Systems Cog Engineer. 


\section{WORKINE COPY}

\subsection{PERFORM 48 HOUR ON-LINE TEST (Cont.)}

Initials/Date

NOTE - During the second hour of operation, the TD shall record the duty cycle of the compressor. This measurement should be done three times and the average noted. Time refers to the approximate time of day the recordings are taken.

5.1.43 MEASURE the time from start of the compression (load) cycle to the end of the compression cycle divided by the total time of one complete load/unload cycle.

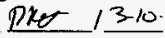

For CMP-801

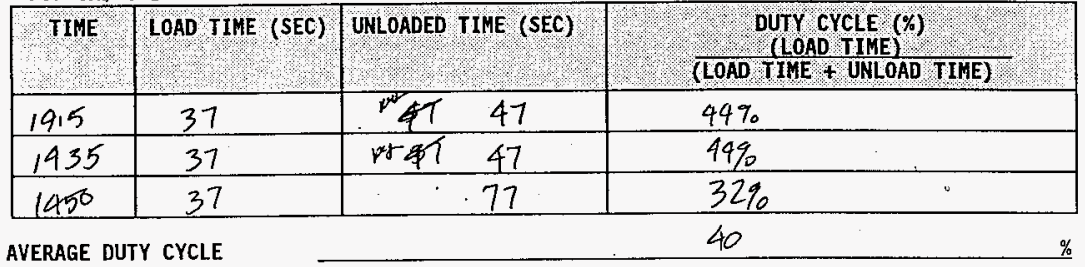




\section{WORKINE COPY}

\subsection{PERFORM 48 HOUR ON-LINE TEST (Cont.)}

Initials/Date

5.1.44 AFTER the first 8 hours of operation, the Stationary Operating Engineer shall RECORD the date and time the system is monitored, the initials of the person taking the readings, and the reading of desired gauges, on Data Sheet every four hours, for the remainder of the 48-hour test.

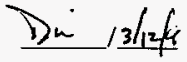

5.1.45 STOP all testing UNTIL Test Director has APPROVED completion of SA-CMP-801 compressor OTP.

Test Director Paldaclo Date 3-12-97

5.1.46 VERIFY air dryer tower switching occurs without malfunctioning.

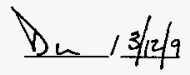

5.1.47 SWITCH compressor SA-CMP-801 to SA-CMP-802 by setting selector switch, SS-1, located on SA-PNL-803 for SA CMP 801 \& 802 SEQUENCER PNL, to SA-CMP-802 (lead unit).

5.1 .48 VERIFY SA-CMP-802 is Lead compressor.

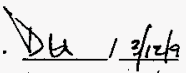

NOTE - SA-CMP-801 should run Unloaded.

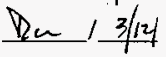

- SA-CMP-801 shall be in the unloaded condition, which starts the $48 \mathrm{hr}$ testing for SA-CMP-802.

- SA-COMP-802 will load and supply air.

5.1.49 POSITION Manual Transfer Switch on GLY-PNL-801 to change Glycol Pump operating.

5.1.50 AFTER approximateiy ten seconds, VERIFY compressor SA-CMP-802 starts.

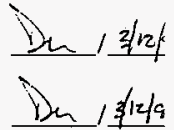

Pump (GLY-P-801 or GLY-P-802) 801

Pump Suction Pressure GLY-PI-801 (PI-701-A-17)

Pump Discharge Pressure GLY-PI-803 (PI-701-A-16)

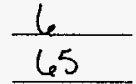

5.1.51 RECORD motor currents in compartment E3 of EDS-MCC-803 for SA-CMP-80\% below:

(A) No Load 60 Amps/Full Load 80 Amps

(B) No Load 60 Amps/Full Load 82 Amps

(C) No Load 60 Amps/Ful1 Load 78 Amps 
5.1.44 AFTER the first 8 hours of operation, the Stationary Operating Engineer shall RECORD the date and time the system is monitored, the initials of the person taking the readings, and the reading of desired gauges, on Data Sheet every four hours, for the remainder of the 48-hour test.

5.1.45 STOP all testing UNTIL Test Director has APPROVED completion of SA-CMP-801 compressor OTP.

Test Director Pueledeullo Date $3-1>-57$

5.1.46 VERIFY air dryer tower switching occurs without mal functioning.

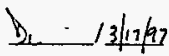

5.1.47 SWITCH Compressor SA-CMP-801 to SA-CMP-802 by setting selector switch, SS-1, located on SA-PNL-803 for SA CMP $801 \& 802$ SEQUENCER PNL, to SA-CMP-802 (lead unit).

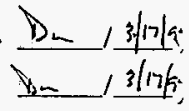

5.1.48 VERIFY SA-CMP-802 is Lead compressor.

NOTE - SA-CMP-801 should run Unloaded.

- SA-CMP-801 shall be in the unloaded condition, which starts the $48 \mathrm{hr}$ testing for SA-CMP-802.

- SA-COMP-802 will load and supply air.

5.1.49 POSITION Manual Transfer Switch on GLY-PNL-801 to change Glycol Pump operating.

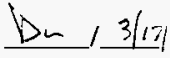

5.1.50 AFTER approximately ten seconds, VERIFY compressor SA-CMP-802 starts.

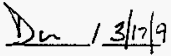

Pump (GLY-P-801 or GLY-P-802) 801

Pump Suction Pressure GLY-PI-801 (PI-701-A-17) 1.5

Pump Discharge Pressure GLY-PI-803 (PI-701-A-16) 55

5.1.51 RECORD motor currents in compartment E3 of EDS-MCC-803 for SA-CMP-802
below:
(A) No Load
cropueson Perugerar pay
(B) No Load Amps Tfull Load Amps
(C) No Load Amps/Fuil Load Amps 


\section{WORKING COPY}

\section{1 PERFORN 48 HOUR ON-LINE TEST (Cont.)}

5.1.52 MONITOR AND RECORD the required items on Data Sheet at 15 minute intervals for the first hour, or unti] the system is stabilized, then continue readings at hourly intervals for the remainder of the first 8 hours.

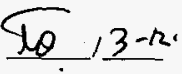

5.1.53 If any unusual conditions are noticed, RECORD the details on an OTP Exception Log sheet and NOTIFY the Test Director and Cog Engineer.

5.1.54 RECORD maximum receiver pressure below, as read from LA-PISL-801. SE

SA-CMP-802 unloads at psig

SA-CMP-802 loads at psig

NOTE - During the second hour of operation, the TD shall record the duty cycle of the compressor. This is done by measuring the time from start of the compression (joad) cycle to the end of the compression cycle divided by the total time of one complete load/unload cycle. This measurement should be done three times and the average noted. Time refers to the time of day the recordings are taken.

5.1.55 MEASURE the time from start of the compression. (load) cycle to the end of the compression cycle divided by the total time of one complete load/unload cycle.

For CMP-802

\begin{tabular}{|c|c|c|c|c|}
\hline TIME & LOAD TIME (SEC) & UNLOADED TIME (SEC) & $\begin{array}{c}\text { DUTY CYCLE (\%) } \\
\text { (LOAD TIME) }\end{array}$ \\
\hline & & & (LOAD THE + ONLOAD THE) \\
\hline & & & \\
\hline
\end{tabular}

AVERAGE DUTY CYCLE $\%$

5.1.56 AFTER the first 8 hours of operation, the Stationary Operating Engineer shall RECORD the date and time the system is monitored, the initials of the person taking the readings, and the reading of desired gauges, on Data Sheet every four hours, for the remainder of the 48-hour test.

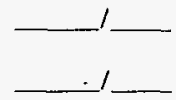

5.1 .56

Section. 5.1 is complete.

Test Director

QC Inspector

Date

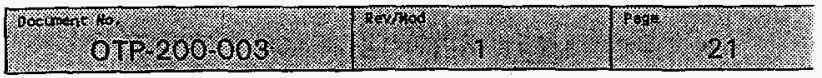


MONITOR AND RECORD the required items on Data Sheet at 15 minute intervals for the first hour, or until the system is stabilized, then continue readings at hourly intervals for the remainder of the first 8 hours.

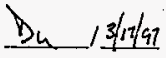

5.1.53 IF any unusual conditions are noticed, RECORD the details on an OTP Exception Log sheet and NOTIFY the Test Director and Cog Engineer.

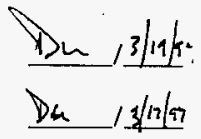

5.1.54 RECORD maximum receiver pressure below, as read from IA-PISL-801.

SA-CMP-802 unloads at 100 psig

SA-CMP-802 loads at 98 psig

NOTE - During the second hour of operation, the TD shall record the duty cycle of the compressor. This is done by measuring the time from start of the compression (load) cycle to the end of the compression cycle divided by the total time of one complete load/unload cycle. This measurement should be done three times and the average noted. Time refers to the time of day the recordings are taken.

5.1.55 MEASURE the time from start of the compression (load) cycle to the end of the compression cycle divided by the total time of one complete load/unload cycle.

PKO / 3r7

For CMP-802

\begin{tabular}{|c|c|c|c|}
\hline TIME & LOAD TIME (SEC) & UNLOADED TIME (SEC) & $\begin{array}{l}\text { DUTY CYCLE (W) } \\
\text { TLOAD TOAD TULE } \\
\text { TWLOAD TIME) }\end{array}$ \\
\hline 1130 & 29 & $4^{\infty} 37$ & $43 \%$ \\
\hline 1133 & 30 & 37 & $44 \%$ \\
\hline 1135 & 19 & 37 & 339 \\
\hline
\end{tabular}

AVERAGE DUTY CYCLE

$$
40
$$
5.1.56 AFTER the first 8 hours of operation, the Stationary
Operating Engineer shal1 RECORD the date and time the system is monitored, the initials of the person taking the readings, and the reading of desired gauges, on Data Sheet every four hours, for the remainder of the 48-hour test.

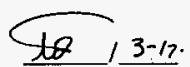

Section 5.1 is complete.

Test Director

Raulf ow w

QC Inspector

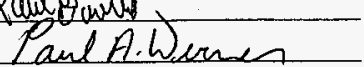

Date $3-19-97$

Date $3-19-97$ 
HNF-SD-WM-OTR-231, Rev 0

Page 54 of 165

\section{NEORMATION COPY}

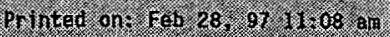

5.2 PERFORM SYSTEM SHUT DOWN

Initials/Date

NOTE - The following steps will stop the permanent compressors

and restart the interim compressors.

5.2.1 START the interim compressor as per instructions posted on interim compressor control panel door.

Len: $13 / \operatorname{cz}_{1} / \varepsilon_{1}$

5.2.2 OPEN valves TAV-4 or TAV-5.

5.2.2.1 POSITYON I-0 (Start-Stop) button on air dryer control panel TAS-1 to "I" position, VERIFY power lamp IS illuminated.

5.2.2.2 ENSURE purge air is set to $45 \mathrm{psi}$.

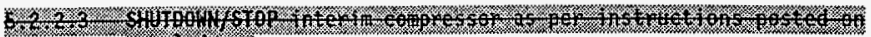

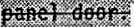

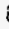

5.2 .3

REPOSITION the following valves:

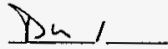

\begin{tabular}{|c|c|c|c|c|c|}
\hline VALVE & POSIIION & INIT & VALVE & BOSITION & INII \\
\hline 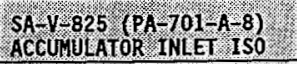 & CLOSED & & $\begin{array}{l}\text { IA-V-857 (IA-702-10) } \\
\text { IA-TK-802 OUTLET ISO VV }\end{array}$ & CLOSEO & \\
\hline 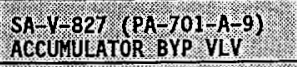 & OPEN & $D_{h}$ & 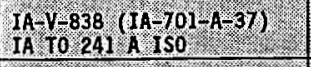 & CUOSED & \\
\hline $\begin{array}{l}\text { SA Y - } 826 \text { (PA-701-A. } 10) \\
\text { ACCUMULATR DUTLET ISO }\end{array}$ & CLOSED & $D_{L}$ & 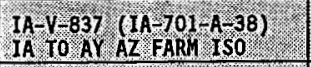 & & \\
\hline 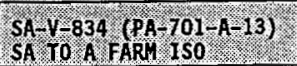 & ClOSEO & D) & 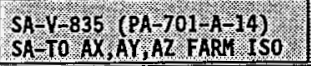 & CLOSED & \\
\hline
\end{tabular}

sete on

5.2.4 REPOSITION the following valves to allow air to flow from the interim compressor to the tank farms:

Dn 1

\begin{tabular}{|c|c|c|c|c|c|}
\hline VILVE & POSITION & INH & VALVE & rositiloN & INT \\
\hline 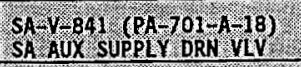 & $B-C$ & Du & $1 A-702-20$ & & \\
\hline $\begin{array}{l}\text { TAV } 10 \text { (OUISIDE) } \\
\text { TEMTORAR AIR SYSTEY }\end{array}$ & OPEN. & Dh & $1 \mathrm{~A}-702-21$ & $\theta=c$ & \\
\hline (1) & 2. & & IA $=702.22$ & B. & \\
\hline
\end{tabular}

* Three-way vaives, normal flow will be parallel to pipe run.

5.2.5 CLOSE valve IA-V-809, IA ORY INLET ISO.

*5.2.8 SEE OCOTON SARET \#4

5.2.6 OPEN IA-V-820, IA DRY BYPASS VLV.

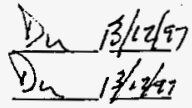




\section{NEORIMATION OOPY}

\subsection{PERFORM SYSTEM SHUT DOWN (Cont.)}

Initials/Date

5.2.7 ALLOW the dryers to cycle and purge the secondary tower. Du / didas

5.2.8 WHEN the pressure gauge reads zero, CLOSE valve IA-V-826, IA DRY OUTLET. ISO.

5.2.9 TURN Dryer power switch to OFF on IA-PNL-805.

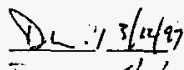

5.2.10 POSITION Manual/Auto switches on SA-PNL-801 and on SA-PNL-802 to the MANUAL position.

5.2.11 PRESS "STOP" button on SA-PNL-801 to stop SA-CMP-801 and

VERIFY that STOP light comes on and compressor stops
running.

VERIFY that STOP light comes on and compressor stops
running.
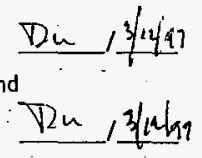

5.2.12 PRESS "STOP" button on SA-PNL-802 to stop SA-CMP-802 and VERIFY that STOP light comes on and compressor stops running.

5.2.13 TURN Pump Control Switch, SW-1, on the GLYCOL CONTROL PANEL, GLY-PNL-801, to OFF.

5.2.14 VERIfY that Glycol Pump is not running.

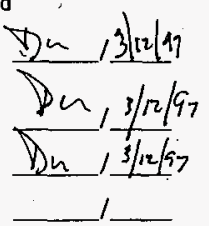

5.2.15 POSITION the following breakers to OFF:

- In Distribution Panel EDS-DP-809 circuit breakers 21,22 and 24 .

- EDS-MCC-803 circuit breaker EDS-CKT-831 in compartment $\mathrm{C} 3$.

- $\quad$ EDS-MCC-803 circuit breaker EDS-CKT-837 in compartment E3.

- EDS-MCC-803 circuit breaker EDS-CKT-844 in compartment GI-R.

5.2.16 POSITION the following disconnects to OFF:

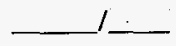

- GLY-DS-801

- GLY-DS-803

- GLY-DS-802

- GLY-DS-804

5.2.17 CHECK the compressors frame oil and ADD oil, if needed.

5.2.18 CHECK AND ADJUST the compressors drive belts per section 9 in the INGERSOLL-RAND PHE MAINTENANCE INSTRUCTION MANUAL.

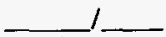


5.2.7 ALLOW the dryers to cycle and purge the secondary tower. Ph , s/19/47

5.2.8 WHEN the pressure gauge reads zero, CLOSE valve IA-V-826, IA DRY OUTLET ISO.

5.2.9 TURN Dryer power switch to OFF on IA-PNL-805.

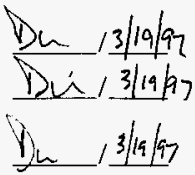

5.2.10 POSITION Manual/Auto switches on SA-PNL-801 and on SA-PNL-802 to the MANUAL position.

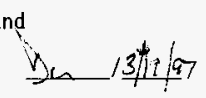

5.2 .12

PRESS "STOP" button on SA-PNL-801 to stop SA-CMP-801 and VERIFY that STOP light comes on and compressor stops running.

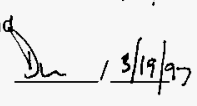

5.2.13 TURN Pump Control Switch, SW-1, on the GLYCOL CONTROL PANEL, GLY-PNL-801, to OFF.

5.2.14 VERIFY that Glycol Pump is not running.

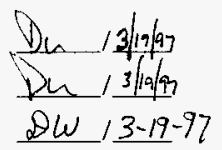

5.2.15 POSITION the following breakers to OFF:

- In Distribution Panel EDS-DP-809 circuit breakers 21,22 and 24 .

- EDS-MCC-803 circuit breaker EDS-CKT-831 in compartment $\mathrm{C} 3$.

- EDS-MCC-803 circuit breaker EDS-CKT-837 in compartment $\mathrm{E} 3$.

- EDS-MCC-803 circuit breaker EDS-CKT-844 in compartment Gl-R.

5.2.16 POSITION the following disconnects to OFF:

$\$ \omega / 3.19 \cdot 97$

- GLY-DS-801

- GLY-DS-803

- GLY-DS-802

- GLY-DS-804

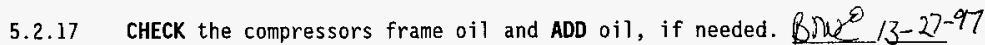

5.2.18 CHECK AND ADJUST the compressors drive belts per section 9 in the INGERSOLL-RAND PHE MAINTENANCE INSTRUCTION MANUAL.
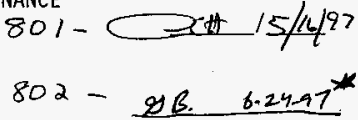

* belts checked with comp. off. 
HNF-SD-WM-OTR-231, Rev 0

Page 57 of 165

WORKING COPY

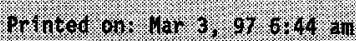

5.2 PERFORM SYSTEM SHUT DOWN (Cont.)

5.2.19 CHECK all of the compressors exterior bolts and nuts AND TIGHTEN if necessary, per INGERSOLL-RAND THE MAINTENANCE INSTRUCTION MANUAL.

5.2.20 CHECK foundation nuts on pipe supports, dryer and receivers, AND TIGHTEN, if necessary, to the Torque as specified by the Test Director.
Initials/Date

$50 \times 13-27^{-97}$

$\left.\operatorname{vof}\right|_{1327-97}$

Section 5.2 is complete.

Test Director Paul \& duello

Date $6-24-97$

QA Inspector

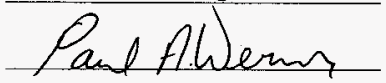

Date $6-24-97$

$600 \%-1 \%$

OTP-200.003

kermes: 1

24 
A11 Operators, Shift Managers, and other personnel performing this Procedure who will be initialing and signing Procedure shall enter their printed name, signature and initials below.

NAME (PRINT)

DR Sones

RH Bowen

B. NEY

T. Kenoutity

Inquishe

R.Jenes -

me Kenbel

$\frac{\text { Lmwaver. Werner (QC) }}{\text { Soul A. }}$

TM Figh
$\forall$ Farley
Bw Eubonks

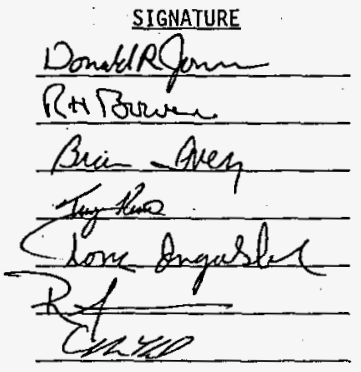

Eapll Werwer

in $t$

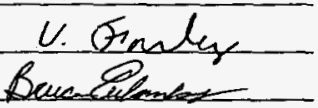

INITIALS

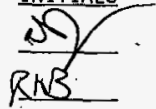

$B A$

$\sum_{10}^{n e}$

RI

me

Lefel

TE

$\frac{U E}{B U E}$
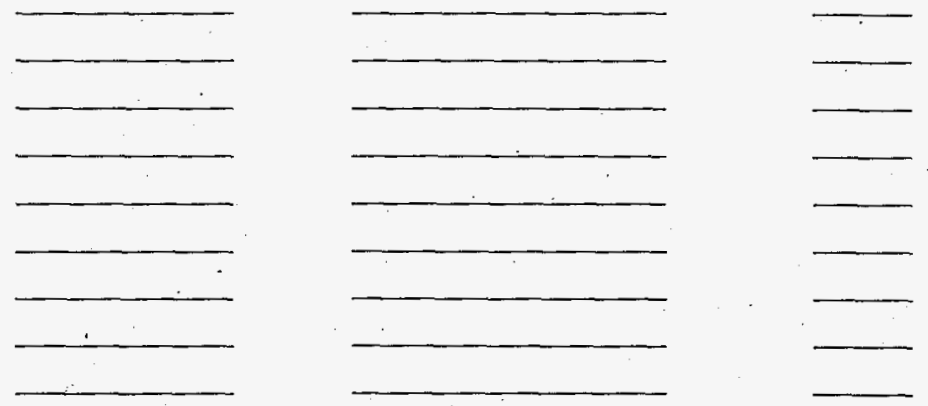
HNF-SD-WM-OTR-231, Rev 0

Page 5 H of 165

$58 A$

WORKING COPY

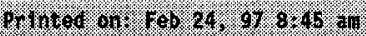

PROCEDURE PERFORMER SIGNATURE SHEET

A11 Operators, Shift Managers, and other personnel performing this Procedure who will be initialing and signing Procedure shall enter their printed name, signature and initials below.

NAME (PRINT)

E. N. WižGjuirR

BI. WUTZKE

MS BRTOEN

DR Jones

Paulgargeco

G Mercer

c. P. CHARLSTON

Wive A. Whin

John C Horne

Bu Funks

DE LDNCEFORD

IE WELLS

WE MEEUWSEN

David Patrick

Glen Anderson

DALE LUNCEFOPO

Greg

David Wile

Robert Jones.
SIGNATURE

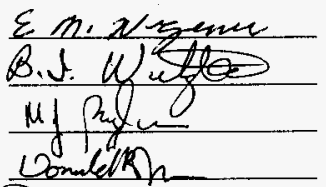

Paultanco

s)lumara

Dap M. Who

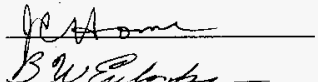

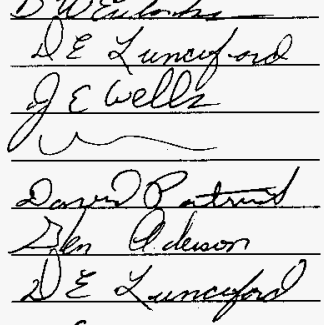

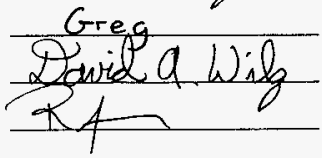

INITIALS

E

bes

MI B

P)21.

Din n

$\frac{C P C}{D h}$

PAt

Bye

$\frac{\frac{d e w}{2 p}}{\frac{d p}{\partial p}}$

$\frac{\text { EA }}{\text { Set }}$

Greg

Daw

R.S.S.

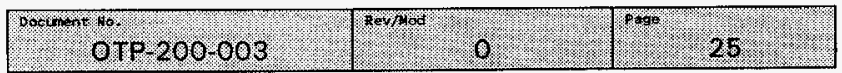




\section{CHECKLIST 1 - COOLING WATER VALVING}

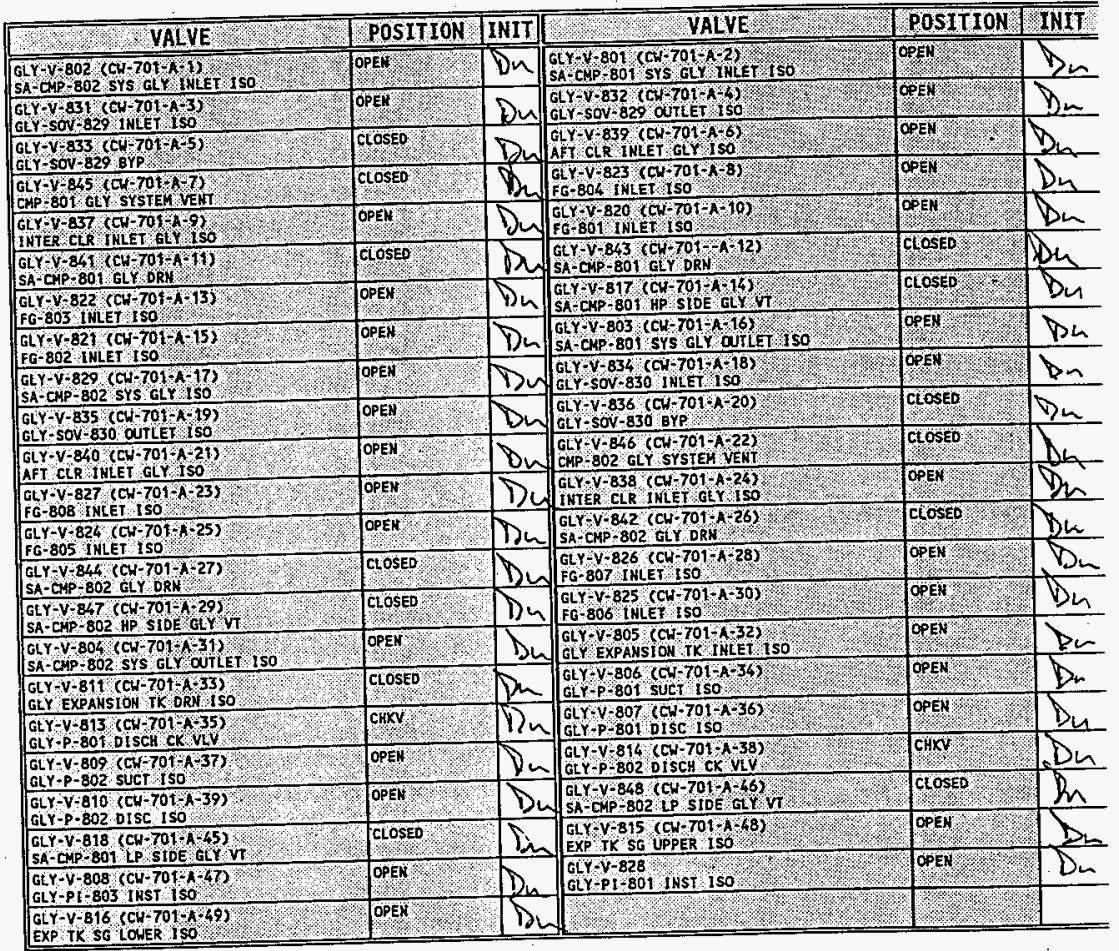




\section{CHECKLIST 1 - COOLING WATER VALVING}

\begin{tabular}{|c|c|c|c|c|c|}
\hline YALVE & POSITION & INIT & VIALVE & POSITION & $\mathrm{INIT}$ \\
\hline $\begin{array}{l}\text { GAY V } 802 \text { (CV-701 AVI) } \\
\text { SA-CMP-802 SYS GLY IMLE I ISO }\end{array}$ & OPEII & & 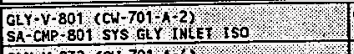 & 0 en & NA \\
\hline $\begin{array}{l}G \mathrm{G} Y \mathrm{Y}-831 \mathrm{(CH-701-A-3)} \\
\mathrm{GLY}-\mathrm{SOV}-829 \mathrm{NLET} \text { ISO }\end{array}$ & OPEF 14 & & 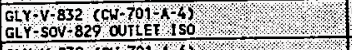 & OPEN & DAA \\
\hline $\begin{array}{l}G\left(Y-V-833\left(C W^{\prime}-701-A / 5\right)\right. \\
G L Y-S O V-829 \text { BYP }\end{array}$ & CIOSED & W & $\begin{array}{l}\text { GLY V }-839(\mathrm{CN} 701 / \mathrm{A}-6) \\
\text { AET CLR INLET } 6 L Y \text { ISO }\end{array}$ & $0 \% \mathrm{~N}, 1$ & \\
\hline $\begin{array}{l}G L Y Y-B 45 \text { (CW-701 A } 7) \\
\text { CMP } 80 \% \text { GLY SYSTEM VENT. }\end{array}$ & CLOSED & & $\begin{array}{l}\mathrm{GLY} Y \mathrm{Y}-823(\mathrm{CH}-701-A-8 \mathrm{j}) \\
\mathrm{FG}-804 \text { iNLEY ISO }\end{array}$ & OPEN & \\
\hline 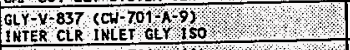 & OPEN & & $\begin{array}{l}G[Y-8-820 \text { (CW } 701)^{-10} \text { ) } \\
F G-801 \text { tNLEI } 150\end{array}$ & OPEN & \\
\hline $\begin{array}{l}\text { GLY-V } 841,(\mathrm{CH}-701-11) \\
\text { SA-CMP } 801 \mathrm{GLY} D \mathrm{DRR}\end{array}$ & CCOSED & & $\begin{array}{l}G L Y-V-8 / 3 \quad\left(C H-701-A^{-12}\right) \\
\text { SA-CMP-BOI GLY DRH }\end{array}$ & Closeb & \\
\hline $\begin{array}{l}\text { GCY - Y } 822\left(\mathrm{CU}-701 \mathrm{~A}^{-13}\right) \\
\text { FG-803 INLET 1SO. }\end{array}$ & OPE & & $\begin{array}{l}\text { GLY-V-817 (CH } 701-A-14) \\
\text { SA-CMP-801 HP SIDE GLY }\end{array}$ & CLOSED & \\
\hline $\begin{array}{l}\text { GLY V }-821 \text { (CN }-701-A \% 15) \\
\text { FG } 802 \text { INLET } 150\end{array}$ & OPEM & & $\begin{array}{l}\text { GLY-V-803 (CH-701-A-96) } \\
\text { SA-CMP-801 SYS GLY OUTLET/ I so }\end{array}$ & Des & \\
\hline 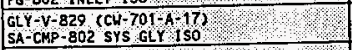 & OPES & & $\begin{array}{l}G L Y-V-834 \text { (CH-701-A-18) } \\
G L Y-S O V-B 30 \text { INLET ISO }\end{array}$ & $09 E N$ & \\
\hline $\begin{array}{l}\text { GLY V- } 835 \text { (CN-701 A } 19) \\
\text { GLY-SOV-B30 OUTLET ISO }\end{array}$ & OPEA & & $\begin{array}{l}\text { GLY-V -836 }(\mathrm{CU}-701-\mathrm{A}-20) \\
\text { GLY-SOV-830 BYP }\end{array}$ & CLOSED & \\
\hline $\begin{array}{l}\text { GYY-V-840 (CW-70t } \mathrm{CAO} 24) \\
\text { AFT CLR INLEI GLY ISO }\end{array}$ & OPEN & & $\begin{array}{l}\text { GLY-V-846 (CH-701-A-22) } \\
\text { CMP-802 GLY SYSTEM VEKT }\end{array}$ & CLOSEO & A \\
\hline $\begin{array}{l}\text { GLY-V-827 (CW-701 } 1-23) \\
\text { FG-808:LNLET ISO }\end{array}$ & OPEN & & $\begin{array}{l}\text { GLY-V } 838 \text { (CU-701-A-24) } \\
\text { INTER CLR INLET GLY I SO }\end{array}$ & OPEN & \\
\hline $\begin{array}{l}\left.\text { GLY V } 824 \text { (CH } 701 A^{-25}\right) \\
F G-805 \text { LMLET IsO }\end{array}$ & OPEN & & $\begin{array}{l}G L Y-V-842(C W-701-A-26) \\
S A-C M F-B O Z \text { GLY DRH }\end{array}$ & CLOOSED & 4 \\
\hline $\begin{array}{l}\text { GLY-V-844 (CW-701-A-27) } \\
\text { SA-CMP-802 GLY DRN }\end{array}$ & CLOSED & & $\begin{array}{l}\text { GLY-V-826 (CiN-701-A-28) } \\
\text { FG-B07 INLET ISO }\end{array}$ & OPEN & A \\
\hline $\begin{array}{l}\text { GLY }-V-847 \text { (CU-701 A-29) } \\
\text { SA-CMP-802 HP SIDE GLYVT }\end{array}$ & CLOSED & & $\begin{array}{l}G L Y-V-825\left(C H / 70^{1}-A-30\right) \\
F G-806 \text { INLET TSO. }\end{array}$ & OPEF & $4 t$ \\
\hline $\begin{array}{l}G 1 Y-V-804 \text { (CW-701-A-31) } \\
\text { SA-CMP } 802 \text { SYS GLY OUTLET } 150\end{array}$ & OPEH & & $\begin{array}{l}G(Y-Y-805(C H-709-\mathrm{A}-32) \\
G L Y \text { EXPAHSIOH TK INLEL I SO }\end{array}$ & OPEN & $A$ \\
\hline $\begin{array}{l}\text { GLY- }-811 \text { (CH } 701-\mathrm{A}-33) \\
\text { GLY EXPANSION TK ORN ISO }\end{array}$ & CLOSED & & $\begin{array}{l}\text { GLY -V-806 (CU-701 A-34), } \\
\text { GLY-P.801 sUCT ISO }\end{array}$ & OPEN & Nt \\
\hline $\begin{array}{l}\text { GLY-V-B13 (CW-701-A } 35) \\
\text { GLY-P-801 DISCH CK VLV }\end{array}$ & C̈̈rV & WA & $\begin{array}{l}\text { GLY-V-807 }\left(\mathrm{CW}-701 \mathrm{~A} \mathrm{~A}^{36)}\right. \\
\text { GLY-P-801 DISC ISO }\end{array}$ & OpEN & \\
\hline $\begin{array}{l}G(Y-Y-B 09 \quad(C H-701 / A-37) \\
G L Y-P-802 \text { sUCr } 150\end{array}$ & OPEN & $10 / 4$ & $\begin{array}{l}\text { GLY-V-814 (CW-701 A-3g) } \\
G L Y-P-802 \text { DISCH CK YLV }\end{array}$ & GHikv & $\sqrt{4}$ \\
\hline $\begin{array}{l}G L Y-V-810 \text { (CH-701-A } 39) \\
G L Y-P-802 \text { oisc IsO }\end{array}$ & OPEN & $g / A$ & 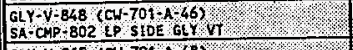 & CLOSED & CA \\
\hline 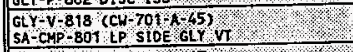 & CLOSED & WA & $\begin{array}{l}\text { GLY-V-815 (CH } 701 \text { A- } 88 \text { ) } \\
\text { EXP TK SC UPPER ISO }\end{array}$ & OQEN & $\operatorname{les}$ \\
\hline 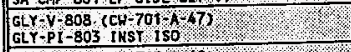 & opgir. & MA & 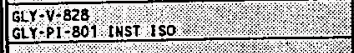 & OPE & 104 \\
\hline 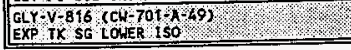 & OPEA & $\mathbb{N} A$ & & & \\
\hline
\end{tabular}


HNF-SD-WM-OTP-231, Rev 0

Page 61 of 165

\section{CHECKLIST 1 - COOLING WATER VALVING}

\begin{tabular}{|c|c|c|c|c|c|}
\hline YALYE & POSITION & INIT & 6. VALVE & POSII ION & TNIT \\
\hline 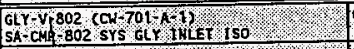 & QPE & 低 & 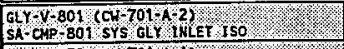 & oper & $\Pi<$ \\
\hline $\begin{array}{l}\text { GLYY } 831 \text { (CVI } 701 \text { A } 3 \text { ) } \\
\text { GLY-SON-829 INLEI } 150\end{array}$ & OPEN & $-1 K$ & 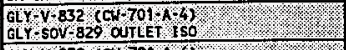 & PPEN & $T K$ \\
\hline 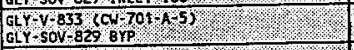 & Clóśg & $T K$ & 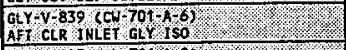 & OPE & $T K$ \\
\hline 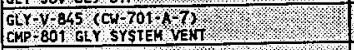 & CCOSEO & & 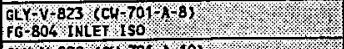 & (OFE & tik \\
\hline 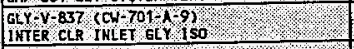 & OPEN & $\pi$ & 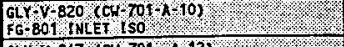 & OPE & $k$ \\
\hline 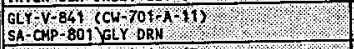 & CLOSES & $\pi$ & $\begin{array}{l}\text { GLY V } 843 \text { ( }(\mathrm{CH}-70 \mathrm{H} / \mathrm{A} / 2) \\
\text { SA-CHP- } 801 \text { GLY ORN }\end{array}$ & $\cos \cos ^{\circ}$ & $T K$ \\
\hline $\begin{array}{l}\text { GLY -V -822 (CH- } 701 / A-13) \\
\text { EG } B 03 \text { INLEI I SO }\end{array}$ & POEM & $7 \mathrm{TC}$ & 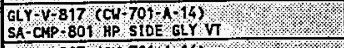 & coosed & $T^{x}$ \\
\hline $\begin{array}{l}G Y Y-821(C H-701-A+15) \\
F G-802 \text { jNLET ISO }\end{array}$ & PPEN Y W & $\pi$ & 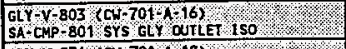 & Oóa & $\pi$ \\
\hline 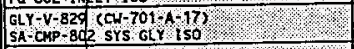 & OPËM & & $\begin{array}{l}\text { GLY Y - } 834 \text { (CU-701-A } 18) \\
\text { GLY-SOV-830 INLET ISO }\end{array}$ & ofen. & $\nabla k$ \\
\hline 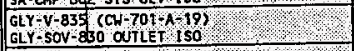 & OPEN & & $\begin{array}{l}\text { GLY-V-836 (CW-701-A-20) } \\
\text { GLY-sov-830 BYP }\end{array}$ & Cioseb: & $\mathbb{R}$ \\
\hline $\begin{array}{l}\text { GLY-V-840 KCU } 701 \text { A } 210 \\
\text { AFI CLR INLET GLY ISO }\end{array}$ & OPEK & $T$ & $\begin{array}{l}\text { GLY-V-846 (cWl701-A-22) } \\
\text { CHP } 802 \text { GLY SYSTEH VEMT }\end{array}$ & CLOSED & $7 k$ \\
\hline $\begin{array}{l}\text { GIY YV-827 (CU-701 } 1-23) \\
F G-808 \text { IMLET ISO }\end{array}$ & OPEN & & $\begin{array}{l}\text { GLY -V-838 (CW-701-A-24) } \\
\text { IKIER CLR INLET GLY I SO }\end{array}$ & OPEN & $\mathbb{R}$ \\
\hline $\begin{array}{l}\left.\text { GLY } \mathrm{V}-824 \text { (CW/701 } \mathrm{A}^{25}\right) \\
\text { FG-805 IMET ISO }\end{array}$ & OPEN & & $\begin{array}{l}\text { GLY }-V-842 \text { (CW } 701-A(26) \\
\text { SA-CMP-802 GLY DRN }\end{array}$ & Closed & $7 K$ \\
\hline $\begin{array}{l}G L Y-V=844,(C 4-701-A-27) \\
5 A-C M P-802 \text { GLY D DRN }\end{array}$ & CLOSED & $\pi<$ & GLY-Y-826 (CH 701-A-28) & OPEX & $T K$ \\
\hline $\begin{array}{l}\text { GLY Y BA7 (CH-701-A } 29) \\
\text { SA-CMP- } 802 \text { HP S SDE GLY VT }\end{array}$ & CLOSED & & $\begin{array}{l}\text { GLY-V-825 (CU-701-A-30) } \\
F G-806 \text { INLET ISO }\end{array}$ & $O P E$ & $\nabla K$ \\
\hline 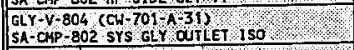 & OPES & & GLY-V-80S (CH-701 A-32) 150 & OPENO & $\nabla / c$ \\
\hline $\begin{array}{l}\text { GLY-V-811 (CW-701-Ae33) } \\
\text { GLY EXPAlNSION IX DRN ISO }\end{array}$ & CLOSED & $\pi c$ & $\begin{array}{l}G L Y-V 806 \text { (CH } 701-A-34) \\
G L Y-P-801 \text { sUCF } 150\end{array}$ & OPEN & $\pi k$ \\
\hline 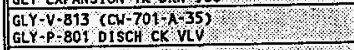 & $\mathrm{CHYV}$ & TK & 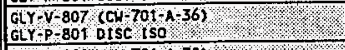 & OPEN & शx \\
\hline $\begin{array}{l}\text { GLY-v-809 (cW-701/k-37) } \\
\text { GLY-P-B02 sucr Iso }\end{array}$ & OPEY & & $\begin{array}{l}G L Y-V-814 \text { (CH-701-A-3B) } \\
G L Y+P-802 \text { Disch CK VLV }\end{array}$ & CHiKV & $\pi k$ \\
\hline 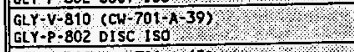 & OPEXY & $\pi^{c}$ & $\begin{array}{l}\text { GLYCV-848 (CH-701A- } 66) \\
\text { SA-CMP-802 LP SIDE ELY VI }\end{array}$ & CLOSED & $\pi$ \\
\hline 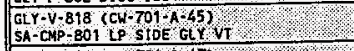 & CTOSED & $-\pi$ & $\begin{array}{l}\text { GLY V-815 (CW-7019-6B) } \\
\text { EXP TK SG UPPER 15O }\end{array}$ & OPEI & $\pi \times$ \\
\hline $\begin{array}{l}\text { GLY-V } 808 \text { (CH- } 701-1-47) \\
\text { GLY-PI-803 IHST TSO }\end{array}$ & OPEN & $T K$ & $\begin{array}{l}\text { GLY-V-828 } \\
\text { GLY-PI-801 IHST } 150\end{array}$ & OPE & $\pi$ \\
\hline $\begin{array}{l}\text { GLY Y } 816 \text { (cul } 701)^{-49)} \\
\text { EXP IK SG LOWER } 1 \mathrm{SO}\end{array}$ & OPEM & & & & \\
\hline
\end{tabular}




\section{CHECKLIST 2 - PROCESS AIR VALVING}

\begin{tabular}{|c|c|c|c|c|c|}
\hline ४VALVE, । & POSITION & INIT & VALVE & POSITION & INII \\
\hline $\begin{array}{l}S A-V-B 12 \text { \&DR }-701-A \\
A D V-8 O 4 \text { OUTLET ISO }\end{array}$ & CEOSED & A & $\begin{array}{l}5 A-V-856\left(0 R-701 / \mathrm{A}^{-3}\right) \\
A D V-801 \text { OR }\end{array}$ & CLOSED & \\
\hline $\begin{array}{l}\text { SA-Y } 862 \text { (ODR-701 }(A-16) \\
\text { SA-ADV-804 BYP V(V }\end{array}$ & Closen & 10 & $\begin{array}{l}\text { SAYVBSS (PA TOT A } 8) \\
\text { ACCUMULATOR TMLET } 150\end{array}$ & COOSED & \\
\hline 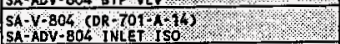 & opery & & 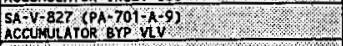 & $0 P E$ & \\
\hline $\begin{array}{l}\text { SA-V-810 (RR-701-A IS) } \\
L G-804 \text { LONER ISO }\end{array}$ & OPEN & & $\begin{array}{l}\text { SAV } 857 \text { (DR } 701, A-11 \text { I } \\
\text { ADV } 802 \text { ORN ISO }\end{array}$ & ClosED & \\
\hline $\begin{array}{l}\text { SA-V } \\
L G=80 \text { UPPER } \text { ISO }\end{array}$ & OPEN & & 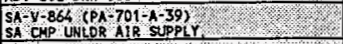 & $\mathrm{OPEH}$ & \\
\hline $\begin{array}{l}5 A-802(G V-7018 A-2) \\
001-802 \text { INST } 150\end{array}$ & OPEN & & 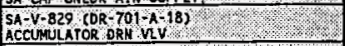 & CLOSEO & \\
\hline $\begin{array}{l}\text { SA-V-816 }(P A-701-A-4) \\
C M P-802 \text { OISCH CK }\end{array}$ & OPEN & & $\begin{array}{l}\text { SA-V } 826 \text { (PA-701-A-10) } \\
\text { ACCLMULATOR OJTLET: }\end{array}$ & ClOSED & \\
\hline $\begin{array}{l}S A-V-815 \text { (PA }-701-A-2) \\
\text { CMP- } 801 \text { DISCH } 150\end{array}$ & OPEX & & $\begin{array}{l}S A-V 830 \text { (GV }-701 / A-4) \\
\text { DISL-801 LNST ISO }\end{array}$ & OPEN & W \\
\hline $\begin{array}{l}\text { SA-V-801 (GV } 701 ; A-1) \\
\text { PDI-801 i NST ISO }\end{array}$ & OPEN & & $\begin{array}{l}\text { SA-Y-B3S }(P A-701-A-14) \\
\text { SA TO AX,AY,AZ FARM } 1 \text { SO }\end{array}$ & CLOSED & \\
\hline $\begin{array}{l}5 A-V-B 21 \quad(G V-701-A-3) \\
P I-807 \text { IKSI } 1 \text { SO }\end{array}$ & OPEN & w & $\begin{array}{l}\text { SA-V-833 (GV }-701-A-5) \\
\text { PI-810 INST ISO }\end{array}$ & OPEN & 4 \\
\hline $\begin{array}{l}S A-V-807 \text { (DR- } 701-A-4) \\
L G-803 \text { UPPER ISO }\end{array}$ & OPEN & & $\begin{array}{l}\text { SA-V } 836 \text { (PA-701-A-15) } \\
S A, \& \text { AX, } Y Y, A Z \text { XCONH }\end{array}$ & CLOSED & \\
\hline $\begin{array}{l}\text { SA-V-809 (DR }-701-A-5) \\
\mathrm{LG}-803 \text { LOWER I SO }\end{array}$ & opeN & & $\begin{array}{l}\text { SA-Y } 834 \text { (PA-701 A-13) } \\
\text { SA TO A FARM ISO }\end{array}$ & CLOSED & $M$ \\
\hline 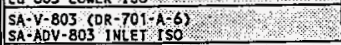 & OPES & & $\begin{array}{l}\text { SA-V-832 (PA-701-A-12) } \\
\text { PCV-801 INEETISO }\end{array}$ & OPEH & 4 \\
\hline $\begin{array}{l}\text { SA-V-86) }(0 R-701-A-8) \\
S A-A D V-803 \text { BYP VLV }\end{array}$ & ctosed & & $\begin{array}{l}\text { SA-V- } 831 \text { (BA-701-A-11) } \\
\text { SA CAP KOR ISO VLV }\end{array}$ & OPEN & \\
\hline $\begin{array}{l}\text { SA-V-811 (OR }-701-A-7) \\
\text { ADV-803 OUTLET ISO }\end{array}$ & ClOSED & & $\begin{array}{l}\text { SAVV } 863 \text { (PA-701 A-40) } \\
\text { EXT AIR INLET } 150\end{array}$ & CLOSED: & \\
\hline $\begin{array}{l}\text { SA'-V }-822(P A-701-A-5) \\
F I-801 \text { INLEI ISO }\end{array}$ & CLOSEO & & $\begin{array}{l}\text { SA-V-BL8 (PA-701-h-24) } \\
\text { FLT-BIS QUTLET TSO }\end{array}$ & CLOSEO & \\
\hline $\begin{array}{l}\text { SA-V } 824(P A-701-A-7) \\
\text { FI-801 BYPASS }\end{array}$ & OPEN & & $\begin{array}{l}\text { SA-V -849 (PA-701 A } 30 \% \\
\text { FLT-819 OUTLEI } 1 \text { SO }\end{array}$ & CLOSED & \\
\hline $\begin{array}{l}\text { SA-V-B23 (PA-701-A-6) } \\
\text { FI-BOT OUTLETISO }\end{array}$ & CLOSED & & $\begin{array}{l}\text { SA-V-852 (PA-701-A-23) } \\
\text { FLT-8IB ORH ISO }\end{array}$ & OPEN & \\
\hline $\begin{array}{l}\text { SA-V-853 }(P A-701 /-29) \\
\text { FLT - } 819 \text { DRN } 150\end{array}$ & OPEN & & $\begin{array}{l}\text { SA-V }-850 \text { (PA-76i }-\mathrm{A}-20) \\
\text { FLT-816 ORH ISO }\end{array}$ & OPEN & \\
\hline $\begin{array}{l}\text { SA }-V=846 \text { ( } 9 A-701-1-22) \\
\text { FLT-B18 INLET I SO }\end{array}$ & OPES & pht & $\begin{array}{l}\text { SAVV-851 (PA-701-A-26) } \\
\text { FLT-817 DRA I SO }\end{array}$ & OPEH & \\
\hline 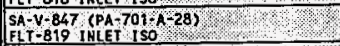 & OPEN & nept & $\begin{array}{l}\text { SA-V-939 }(P A-701-A(16)- \\
\text { SA AUX SUPPEY I SO }\end{array}$ & OPEY & \\
\hline 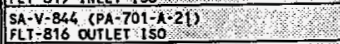 & $O P E N$ & & $\begin{array}{l}\text { SA-V-841 (PA-701 - } \\
\text { SA AUX SUPPLY DRA VIV }\end{array}$ & $B C$ & \\
\hline $\begin{array}{l}\text { SA-V } 845 \text { (PA } 701-\mathrm{A} 27) \\
\text { FLI-817 ouTLET tSO }\end{array}$ & $O P E Y$ & IOA & $\begin{array}{l}8 A-Y-839 \text { (PA-70TA A 31) } \\
\text { SA AUX SUPPLY ISO }\end{array}$ & CLOSED & \$ \\
\hline $\begin{array}{l}\text { SA-V-842 }(P A-701 / \mathrm{A}-19) \\
\text { FLT- } 816 \text { iNLEI 1 So }\end{array}$ & CLOSED & d4t & $\begin{array}{l}\text { SA Y } 828 \text { (OR } 701 \text { A } 17)^{4} \\
\text { ACCUULATOR DRH YLV }\end{array}$ & GPE & $1 N$ \\
\hline 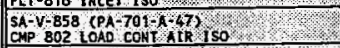 & OPE & dit & 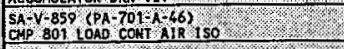 & $O P E A$ & ast \\
\hline $\begin{array}{l}5 A-V-843 \text { (PA-701\% } \\
\text { FLT-817 I HLET 1 } 250\end{array}$ & CLOSEO & $\mathrm{CAA}$ & & & \\
\hline
\end{tabular}

* Three-way valves, normal flow is $A-B$, alternate flow $B-C$.

** Located in zone under Receiver Tank and verified OPEN by actuating SA-V-829. 


\section{CHECKLIST 2 - PROCESS AIR VALVING}

\begin{tabular}{|c|c|c|c|c|c|}
\hline$\%, \quad$ & POSITION & IT & Y YALYE & POSITION & $\mathrm{T}$ \\
\hline 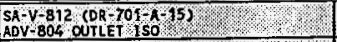 & CLOSED & & 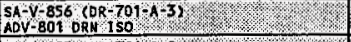 & CLOSEO & \\
\hline 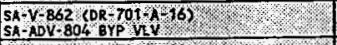 & ClOSED & & $\begin{array}{l}S A-\sqrt{825}(\mathrm{PA}-701-\mathrm{A}-\mathrm{s}) \\
\text { ACCUMULATOR (NLET ISO }\end{array}$ & closgo & \\
\hline 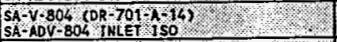 & OPEN & & $\begin{array}{l}\text { SA-V } 827 \text { (PA.701 A } 9) \\
\text { ACCUAULATOR BYP VLY }\end{array}$ & OpEit & \\
\hline $\begin{array}{l}S A-810 \text { (DR }-701 / A^{-13)} \\
L Q=804 \text { LOWER ISO }\end{array}$ & OPES & & $\begin{array}{l}5 A-857 \text { (QR } 701 \% 11 \text {. } \\
A D V-802 \text { DRH ISO }\end{array}$ & $C F O S=0$ & \\
\hline 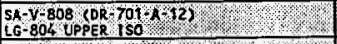 & SFEF & & 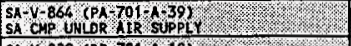 & Gper & \\
\hline $\begin{array}{l}3 A-802 \text { (GV } 701 / \mathrm{A}) \\
\text { POI } 802 \text { INST } 150 .\end{array}$ & OPEY & & 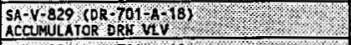 & giosep & \\
\hline $\begin{array}{l}\text { SA-V816 (PA-701 A } \\
\text { CKP-802 orsch cK }\end{array}$ & $O P E N$ & & $\begin{array}{l}\text { SA-V-826 (PA-701) A-10) } \\
\text { ACQUMULATOR OUTEE ISO }\end{array}$ & CCOSEO & \\
\hline 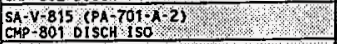 & $O P E M$ & & 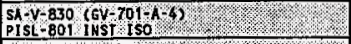 & OpeN & \\
\hline 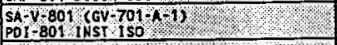 & OQE & & 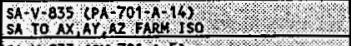 & COOSED & \\
\hline $\begin{array}{l}\text { SA-V } 821 \text { (GV } 701-\mathrm{A}-3) \\
P I=807 \text { INST } 150\end{array}$ & OPEN & & $\begin{array}{l}S A-V-833 \text { (GV } 70 A^{-5} \\
P I-810 \text { I NST I SO }\end{array}$ & OPEI & \\
\hline $\begin{array}{l}\text { SA-V-807 (DR-701 A-4) } \\
\angle G-803 \text { UPQER ISO }\end{array}$ & OPEY & & $\begin{array}{l}S A-Y-836 \text { (PA-701 A } 15) \\
\text { SA A \& , AY,AZ XCONH }\end{array}$ & CLOSED & \\
\hline $\begin{array}{l}\text { SA-V } 809 \text { (DR }-701 / A 5) \\
\angle G-803 \text { LOLER TSO }\end{array}$ & OPEN & & $\begin{array}{l}\text { SA-V-834 (PA-701 A } 13) \\
\text { SA IO A FARM } 150\end{array}$ & closeo & \\
\hline $\begin{array}{l}\text { SA- } Y-803 \text { GDR } 70(-A-6) \\
\text { SA-ADV-803 INLET ISO }\end{array}$ & OPEN & & $\begin{array}{l}\text { SA-V-832 } P A-701-A 12) \\
P C V-801 \text { I NLET ISO }\end{array}$ & OPEM & \\
\hline $\begin{array}{l}\text { SA-V B61 }(D R-701 / A B) \\
\text { SA-ADV } 803 \text { BYP VLV }\end{array}$ & CLOSEO & & $\begin{array}{l}\text { SA } V-831 \\
\text { SA CMP HDR }\left(P A-701-\mathrm{Al}^{-1 \mathrm{O}}\right)\end{array}$ & OPEY & \\
\hline $\begin{array}{l}\text { SA-V } 811 \text { (DR-701/ } \\
\text { AOV-803 OUTLET ISO }\end{array}$ & CLOSED & & $\begin{array}{l}\text { SA-V - } 863 \text { SPA-701 A- } 60) \\
\text { EXT AIR SALET } 1 \text { SO }\end{array}$ & CLOSEO & \\
\hline $\begin{array}{l}\text { SA }-822 \text { (PA } 701-A) \\
\text { FI-8OT IHLET I SO }\end{array}$ & CCOSED & & $\begin{array}{l}\text { SA-V } 848 \text { (PAC70I-K-24) } \\
\text { FLT-8 } 8 \text { 18 OUTLET ISO }\end{array}$ & CLOSED & \\
\hline $\begin{array}{l}S A-Y-824 \text { (PA-701 A } 7) \\
F I-801 \text { BYPASS }\end{array}$ & OPEN & & $\begin{array}{l}\text { SA-V } 849 \text { (PA-7O1 A } \\
\text { FLI-B } 30 \text { OUTLET ISO }\end{array}$ & $\mathrm{CCOSEO}$ & \\
\hline $\begin{array}{l}S A-V_{1}^{823}(P A-701-A-6) \\
F 1-801 \text { OUTLET I SO }\end{array}$ & CLOSED & & $\begin{array}{l}S A-V-852 \text { (PA-701 A-23) } \\
F L T-818 \text { DRA } 1 \text { SO }\end{array}$ & $O P E N$ & \\
\hline $\begin{array}{l}\text { SA-V } 8853 \text { (PA-701 }-1-29) \\
F L T-819 \text { DRN I ISO }\end{array}$ & OPEN & & $\begin{array}{l}\text { SA- }-850(P A-701-20) \\
\text { FLI }-816 \text { DRN I SO }\end{array}$ & OPEY & \\
\hline $\begin{array}{l}\text { SA-V-846 (PA-701-A } 22) \\
\text { FLT-81a IMLET ISO }\end{array}$ & OPEN & & $\begin{array}{l}S A-V-851 \quad(P A-70)^{-A-26)} \\
F L T-817 \text { DRN } 1 \text { SO }\end{array}$ & OPEN & \\
\hline $\begin{array}{l}\text { SA- }-847 \text { (PA-701-A } 28) \\
\text { FLT-819 IMCT I SO }\end{array}$ & OPEN & & $\begin{array}{l}\text { SA- } V \text { - } 838 \text { (PA } 701-\mathrm{A}^{-16)} \\
\text { SA AUX SUPPLY ISO }\end{array}$ & OPEA & \\
\hline $\begin{array}{l}\text { SA-V-B44 (PA-701-A-21) } \\
\text { FLT 816 OUTLETISO }\end{array}$ & OPEN & & $\begin{array}{l}\text { SA-V-84 (PA 701 A } 18) \\
\text { SA AUX SLPPEY ORA VVV }\end{array}$ & $B C$ & \\
\hline $\begin{array}{l}\text { SA V-845 (PA-701-1-27) } \\
\text { FLT-817 OUTLET LSO }\end{array}$ & OPEF & & $\begin{array}{l}\text { SA-V-839 (fPA-701 A } 31)^{\circ} \\
\text { SA ALX SUPPLY ISO }\end{array}$ & CLOSED & \\
\hline 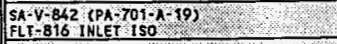 & COOSEO & & $\begin{array}{l}\left.\text { SA-V-828 COR } 701-\mathrm{Al})^{2}\right)^{\circ} \\
\text { ACCUMULATOR DRN VLV }\end{array}$ & $\mathrm{oper}$ & \\
\hline $\begin{array}{l}\text { SA-V-858 (PA-701/A-47) } \\
\text { CMP } 802 \text { LOAD CONT AIR ISO }\end{array}$ & opes: & & $\begin{array}{l}\text { SA-Y-859 \&PA-701-A AGS } \\
\text { CMP } 801 \text { LOAD CONT AR } 150\end{array}$ & OPEM & \\
\hline $\begin{array}{l}\text { SA-Y } 843 \text { (PATOOI } \\
\text { FLT-817 INLET ISO }\end{array}$ & ClOSED & & & 18 & \\
\hline
\end{tabular}

* Three-way valves, normal flow is $A-B$, alternate flow B-C.

** Located in zone under Receiver Tank and verified OPEN by actuating SA-V-829. 
HNF-SD-WM-OTR-231, Rev 0

Page 64 of 165

\section{CHECKLIST 2 - PROCESS AIR VALVING}

\begin{tabular}{|c|c|c|c|c|c|}
\hline VALVE & POSITION & INIT & VALVE & BOSITION & INIT \\
\hline 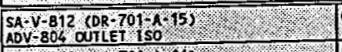 & Closed & $T F$ & $\begin{array}{l}\left.\text { SAVV-856 (DR } 7011^{-3}\right) \\
\text { ADV-801 ORN ISO }\end{array}$ & cooseo & Rc \\
\hline $\begin{array}{l}5 A+Y-862 \quad(0 R-701 \text { A } 16) \\
S A-A D V-804 \text { BYP }\end{array}$ & Closen: & TK & 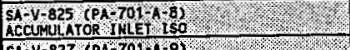 & CLOSD & Ths \\
\hline 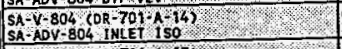 & OPEN & $\pi$ & 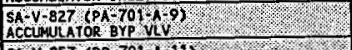 & OPEM & $\pi c$ \\
\hline 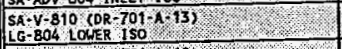 & OPEW & Th & 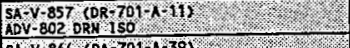 & Close & TK \\
\hline $\begin{array}{l}S A-V B O 8 \text { (OB } 701 / 19 \\
L G-804 \text { UPPER ISO }\end{array}$ & OPEN & 10 & 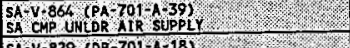 & opur & $\pi c$ \\
\hline 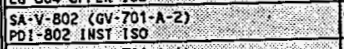 & OPEK & $-\mathfrak{K}^{\mathrm{C}}$ & 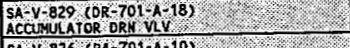 & CLOSED & \\
\hline $\begin{array}{l}S A-V-816 \quad(P A-701 / A-4) \\
C M P-802 \text { D1SCH CK }\end{array}$ & OPEN & $\pi c$ & 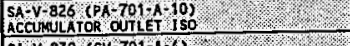 & CLosed & $+k$ \\
\hline $\begin{array}{l}5 A-y-815(P A-701-A-2) \\
0 A P-801 \text { o1scif } 150\end{array}$ & OPEH & $\pi^{C}$ & $\begin{array}{l}\text { SAY }-830 \text { (GV } 701 \mathrm{~A}^{\mathrm{A}}-\mathrm{S} \\
\text { PISL-801 INSI LSO }\end{array}$ & OPES & $t c$ \\
\hline $\begin{array}{l}\left.5 A-V-B 01 \text { (GV- } 701 A^{-1}\right) \\
P D 1-802 \text { INST } 150\end{array}$ & OPEN & $\pi$ & $\begin{array}{l}\text { SA- }-235 \text { \% } P A-701 \text { A } 14) \\
\text { SA TO AX,AYAZ FARE ISO }\end{array}$ & closst & $T R C$ \\
\hline $\begin{array}{l}5 A-V-821 \text { (GV-701-A } \\
P 1-807 \text { INST i SO }\end{array}$ & OPEN & DC & $\begin{array}{l}S A-Y-B 33 \text { (oV } 701-1-5) \\
\text { PI-B10 IUST } 1 \text { SO }\end{array}$ & OPE & $\pi$ \\
\hline $\begin{array}{l}5 A-Y-807 \text { (DR } 701-A-4) \\
\angle G-803 \text { UPPER ISO }\end{array}$ & OPEF & $T H$ & $\begin{array}{l}\text { SA-V-836 (PA-701 1A-15) } \\
\text { SA \& AX, XY, XCONH }\end{array}$ & CLOSED & $\pi$ \\
\hline $\begin{array}{l}\text { SA-Y } 8009(\mathrm{DR}-701-A 5) \\
\text { LO-803 LOUER ISO }\end{array}$ & OPEI & $14 c$ & $\begin{array}{l}\text { SA-9-834 (PA-701-A-13) } \\
\text { SA YOA FARM ISO }\end{array}$ & CLOSO & 97 \\
\hline $\begin{array}{l}\text { SA-V }-803 \text { (DR-701-A-6) } \\
\text { SA ADV } B O 3 \text { I ILET ISO }\end{array}$ & OPEY & 110 & $\begin{array}{l}\text { SA-V -BJ2 SPA-701 A } 12) \\
\text { PCV-801 } 1 N L E Y \text { ISO }\end{array}$ & OPEF & t \\
\hline $\begin{array}{l}5 A-Y<B 1 \quad(D R-701 / A-8) \\
S A-A D V-803 \text { BYP V }\end{array}$ & CLOSEO & $\pi L$ & $\begin{array}{l}\text { SA-Y-831 (PA-701-A-11) } \\
\text { SA CMP HOR ISO YLV }\end{array}$ & oper & $+k$ \\
\hline $\begin{array}{l}S A-V-811 \text { (OR-701 A } 7) \\
\text { ADV-803 ONTLET ISO }\end{array}$ & CCOSEO & $\pi$ & $\begin{array}{l}5 A-V-863 \text { (PA-701-A-40) } \\
\text { EXI AIR INLET 1SO }\end{array}$ & CloseO & $7 \mathrm{r}$ \\
\hline $\begin{array}{l}\text { SA-V- } 822 \text { (PA- } 701 \%-5) \\
\text { FI-801 INLEI ISO }\end{array}$ & CLOSED & $\pi$ & $\begin{array}{l}\text { SA-Y-848 (PA-701-A-24) } \\
\text { FLT-B1B QUTLET ISO }\end{array}$ & CLOSED & \\
\hline $\begin{array}{l}S A-\mathrm{V}-824(\mathrm{PA}-701-\mathrm{A}) \\
\text { FI-801 BYPASS }\end{array}$ & OPEH & $\overline{T K}$ & $\begin{array}{l}S A-V-849 \text { (PA-TO }-\mathrm{A}-30) \\
F L T-879 \text { oUILE } 1 \mathrm{SO}\end{array}$ & CLOSEO & $\pi c$ \\
\hline SAY-823 (PA-701-A 6 ) & Closed & $x$ & $\begin{array}{l}\text { SA-V-852 (PA } 701-A-23) \\
F L T-818 \text { DRA I SO }\end{array}$ & $O P E H$ & $\pi 4$ \\
\hline $\begin{array}{l}S A-V-853 \text { (PA-70 } 1-A-29) \\
\text { ELT-819 DRN ISO }\end{array}$ & OPEN & $\pi^{c}$ & $\begin{array}{l}S A \cup V-850 \text { (PA-701-A-20) } \\
\text { ELT } 816 \text { ORA } t S O\end{array}$ & OPEY & $k$ \\
\hline $\begin{array}{l}S A-V-B 46 \quad(F A-701-822) \\
F L T-818 \text { IHLET ISO }\end{array}$ & OPEN & $-\pi$ & $\begin{array}{l}S A-V-851 \text { (PA } 701 / A-26) \\
F L T-817 \text { DRA ISO }\end{array}$ & OPEN & $\pi$ \\
\hline $\begin{array}{l}\text { SA-V \&47 (PA-701 A-28) } \\
\text { FLT } 819 \text { I NLET I SO }\end{array}$ & OPEK & $\pi$ & $\begin{array}{l}\text { SA-Y } 838 \text { (PA-701 A-16)\% } \\
\text { SA, AUX SUPPLY ISO }\end{array}$ & OPEN & $T i c$ \\
\hline $\begin{array}{l}S A-\hat{Y}-844 \text { CPA-701 A-21) } \\
\text { FL-816 OULET ISO }\end{array}$ & OPES & $\pi k$ & $\begin{array}{l}\text { SA-V } 841 \text { (PA-701 A } 1 B)- \\
\text { SA AUX SUPPLY DRA VUV }\end{array}$ & $B C$ & $7 k$ \\
\hline $\begin{array}{l}5 A-V-845 \text { (PA } 701-A-27) \\
F L T-B 97 \text { OULET 1SO }\end{array}$ & OPEM & $\pi$ & $\begin{array}{l}\text { SA-Y } 839 \text { (PA-701 } 1-31) \\
\text { SA AUX SUPPLY ISO }\end{array}$ & Close & $T_{s}$ \\
\hline 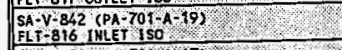 & CLOSED & $\pi c$ & $\begin{array}{l}\text { SA-V } 828 \text { (OR } 701 \text { A A } 17)^{\circ} \\
\text { ACCUMULATOR ORA VLV }\end{array}$ & Open & k \\
\hline $\begin{array}{l}\text { SA-Y } 858 \text { (PA-701 A A } 7) \\
\text { CMP 802 LOAD CONTAR ISO }\end{array}$ & OPEX & $\pi^{c}$ & 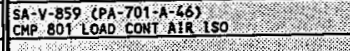 & opert & TK \\
\hline $\begin{array}{l}\text { SA } Y-843 \text { (FA } 701-25) \\
\text { FLI-8 } 27 \text {, NLEI SO }\end{array}$ & Ccosed & 7 & & & \\
\hline
\end{tabular}

* Three-way valves, normal flow is $A-B$, alternate flow B-C.

** Located in zone under Receiver Tank and verified OPEN by actuating $\$ A-V-829$. 


\section{CHECKLIST 2 - PROCESS AIR VALVING}

\begin{tabular}{|c|c|c|c|c|c|}
\hline ४० YALVE & POSITION & INIT & VALVE & POSITION & \\
\hline $\begin{array}{l}\text { SAAV } 812 \text { (DR-7O1 A } 15 \text { ) } \\
\text { AOV }=804 \text { OUTLET } 1 \text { SO }\end{array}$ & Closed: & $D$ & $\begin{array}{l}\text { SA Y } 856 \quad(D R-701-3) \\
\text { ADV-801 DR } 150\end{array}$ & $\cos \varepsilon 0$ & \\
\hline $\begin{array}{l}\text { SA-Y } 862 \text { (OR-7OT-A } 16) \\
\text { SA-AOV-804 BYP VLV }\end{array}$ & Cosed & & 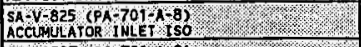 & ClOSED & \\
\hline 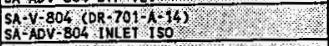 & OPE & $\theta$ & 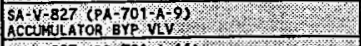 & OPE & \\
\hline $\begin{array}{l}5 A-810 \text { (OR } 701 / \mathrm{A} / 3) \\
L G .804 \text { LONER ISO }\end{array}$ & OPEF & & $\begin{array}{l}S A-V-857 \text { (DR } 701.1-11) \\
A D V-802 \text { DRN ISO }\end{array}$ & closed & \\
\hline $\begin{array}{l}S A Y-B 08 \text { (DR-701-A-12) } \\
L G-804 \text { UPFER ISO }\end{array}$ & OPEN & & $\begin{array}{l}\text { SA }-864 \text { (PA-701 A } 39) \\
\text { SA CMP UNLOR AIR SUPPEL }\end{array}$ & $\mathrm{OPE}$ & \\
\hline $\begin{array}{l}\mathrm{SA}-\mathrm{V}-802 \mathrm{6GV}-701 / \mathrm{A} 2 \mathrm{~V} \\
\mathrm{PD} 1-802 \text { 1 }\end{array}$ & OPEH & & 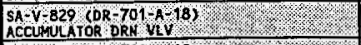 & CLOSEP & \\
\hline $\begin{array}{l}\mathrm{SAY-816}(\mathrm{PA}-701-4-6) \\
0 \mathrm{PH}-802 \text { oISCH CK }\end{array}$ & OPEN & & $\begin{array}{l}S A-y-826 \text { (PA } 701-1-10) \\
\text { ACCUMLATOR OUTLET ISO }\end{array}$ & COOSED & \\
\hline 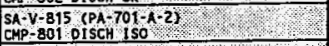 & opes & & $\begin{array}{l}\left.\text { SA-Y } 830 \text { ( } \mathrm{GV} 701 \mathrm{~A}^{4}\right) \\
\text { PISt } 801 \text { UNST tso }\end{array}$ & OPEH & \\
\hline $\begin{array}{l}\text { SAY } 801 \text { (GV }-701-A-1) \\
\text { POI } 801 \text { INST } 150\end{array}$ & OPEI & & 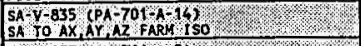 & CLOSE & \\
\hline $\begin{array}{l}\text { SA-V-821 (GV } 701 \mathrm{~A}^{-3} \text { ) } \\
\text { PI-BOT INSI (SO }\end{array}$ & OPEY & & $\begin{array}{l}S A-V-833 \text { (GV } 701-A-5) \\
\text { PI-810 INSI ISO }\end{array}$ & $O P E$ & \\
\hline $\begin{array}{l}\text { SA- }-807 \text { (DR }-701-A^{-4)} \\
\text { LG-803 UPPER ISO }\end{array}$ & $O P E M$ & & $\begin{array}{l}\text { SA-Y \&36 (PA } 701 \text { 1 A } 15) \\
\text { SA } A \text { \& AX AY,AZ XCONA }\end{array}$ & CLOSED & \\
\hline $\begin{array}{l}S A-Y-809 \text { ? } \\
L G-803 L O W\end{array}$ & OPEN & & $\begin{array}{l}\text { SA-V }-834 \text { (PA } 701-A-13) \\
\text { SA TO A FARM ISO }\end{array}$ & ClOSEO & \\
\hline $\begin{array}{l}S A-V=803 \text { COR-7 } \\
S A-A D V=803 \text { IHA }\end{array}$ & OPEN & & $\begin{array}{l}\text { SA-V }-832 \text { (PA-701 A-12) } \\
\text { PCV } 80 \text { INLEI I SO }\end{array}$ & OPEY & \\
\hline $\begin{array}{l}\text { SA-V-8S1 }(0 R-701-A-8) \\
\text { SA-ADV-803 BYP viV? }\end{array}$ & CLOSED & & $\begin{array}{l}\text { SA-V-831 (PA-701-A-1 } \\
\text { SA CMP HDR } 150 \text { VLV }\end{array}$ & $\mathrm{opeN}$ & \\
\hline $\begin{array}{l}5 A-Y-811 \text { (DR } 701 \text { A }-7) \\
A D V-803 \text { OTTLET ISO }\end{array}$ & CLOSED & & $\begin{array}{l}\text { SA-V } 863 \text { (PA-701-A-60) } \\
\text { EXI AIR INLET I SO }\end{array}$ & CLOSED & \\
\hline $\begin{array}{l}\text { SA-Y-B2Z (PA-701 } / A-5) \\
\text { FI-BOT LNLEI ISO }\end{array}$ & closeo & & $\begin{array}{l}\text { SA-V-849 (PA-701 A-24) } \\
\text { FLT } 818 \text { OULEI ISO }\end{array}$ & Closea & \\
\hline $\begin{array}{l}\text { SA- }-824 \text { (PA-701-A-7) } \\
\text { FI } 801 \text { BYPASS }\end{array}$ & DOPEH & & $\begin{array}{l}\text { SA Y } 849 \text { (PA } 70) / 30) \\
\text { FL } 819 \text { OUTLET }\end{array}$ & Closeo & \\
\hline $\begin{array}{l}S A-V-823(P A-701-A-6) \\
\text { E1-801 OUTLEE } 150\end{array}$ & CLOSED & & $\begin{array}{l}\text { SA } Y-852 \quad\left(\mathrm{PA}_{1}-701-\mathrm{A}^{23}\right) \\
\text { FET-BIE DRN ISO }\end{array}$ & $\mathrm{OPEN}$ & \\
\hline $\begin{array}{l}\text { SA-V-853 (PA } 701-A-29) \\
\text { FLT-819 DRN ISO }\end{array}$ & LPEH & & $\begin{array}{l}\left.\text { SA-V }-850 \text { (PA-701 }{ }^{-20}\right) \\
\text { FET-816 ORA ISO }\end{array}$ & OPEI & \\
\hline $\begin{array}{l}\text { SA-V } V=846 \quad(P A-701-22) \\
F L T-818 \text { INLET ISO }\end{array}$ & OPEN & & SA V - 851 (PA-701-A 26$)$ & OPE & \\
\hline $\begin{array}{l}\text { SA-V }=847 \text { (PA-701-A-28) } \\
\text { ELT-819 I ILET ISO }\end{array}$ & OPEK & D & $\begin{array}{l}\text { SA- V-838 }(P A-701, A-16)^{*} \\
\text { SA NUX SUPPLY ISO }\end{array}$ & OPEY & \\
\hline $\begin{array}{l}\text { SA-V-844 (PA-701 A-29) } \\
\text { FLT-816 aUTLET I SO }\end{array}$ & OOEY & D) & $\begin{array}{l}\text { SA-V }-841 \text { (PA-701 AA 18) } \\
\text { SA AUX SUPPEY ORA VUV }\end{array}$ & 80 & \\
\hline $\begin{array}{l}\text { SA-V } 845 \text { CPA } 701 / 27 \\
\text { FLT-817 OUTLEI ISO }\end{array}$ & $O P E Q_{1}$ & D & $\begin{array}{l}\text { SA- Y } 839 \text { (PA-701- }{ }^{-31)} \\
\text { SA AUX SUPPCY I SO. }\end{array}$ & CLOSED & \\
\hline $\begin{array}{l}\text { SA-V }-842 \text { (PA-701-A-19) } \\
\text { FL- }-316 \text { IHLET ISO }\end{array}$ & CLOSED & $\bar{D}$ & 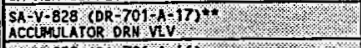 & OPEN & \\
\hline 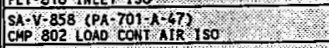 & opeir. & D. & 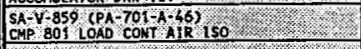 & OPE & D \\
\hline $\begin{array}{l}\text { SA-V }-843 \text { IPA-701 A } 25) \\
\text { Fi: I-817 INLEI ISO }\end{array}$ & $C \operatorname{COS}=0$ & & & & \\
\hline
\end{tabular}

* Three-way valves, normal flow is $A-B$, alternate flow B-C.

$\therefore *$ Located in zone under Receiver Tank and verified OPEN by actuating SA-V-829. 


\section{CHECKLIST 3 - INSTRUMENT AIR VALVING}

\begin{tabular}{|c|c|c|c|c|c|}
\hline VALVE & POSI & & VALVE & POS & $2 \mathrm{NA}$ \\
\hline $\begin{array}{l}\text { IA-Y } 837 \text { (IA } 701 \text { A-38) } \\
\text { IA TO AY AZ FARM ISO }\end{array}$ & CLOOS & T) & $\begin{array}{l}\text { SA-V } 8554 \text { (IA-701-A } \\
\text { FLT-814 INLET ISO }\end{array}$ & OPE & 6 \\
\hline $\begin{array}{l}\text { IA-V }-841 \text { (GY } 701-A-8) \\
\text { A PI } 822 \text { [SO }\end{array}$ & & 厉 & $\begin{array}{l}\text { SA-V-855 (IA-701-A-7) } \\
\text { FET-815 INLET ISO). }\end{array}$ & $\cos$ & \\
\hline $\begin{array}{l}* \text { SA-Y }-860 \text { (IA-701-A-36) } \\
\text { SA FLT SYS BYP VLV }\end{array}$ & CLOSED & & $\begin{array}{l}1 A-V-820 \text { (IA-701-A } 12) \\
\text { IA DRY BYPASS VLV }\end{array}$ & OPEN & \\
\hline $\begin{array}{l}\text { IA-V-838 (IA-701-A-37) } \\
\text { IA T0 241 A } 150\end{array}$ & CLOSED & & $\begin{array}{l}\text { IA-V } 810 \text { (IA-70I / A I5) } \\
\text { IA FLT } 816 \text { INLET ISO }\end{array}$ & & \\
\hline $\begin{array}{l}\mathrm{IA} V=842 \text { (GV } 701-\mathrm{A}-7 \text { ) } \\
\mathrm{IA} \mathrm{PI} 823 \text { IS0 }\end{array}$ & OPEN/2 & & $\begin{array}{l}\text { IA-V-812 (IA-701-A-19) } \\
\text { IA FLT 816 OUTLET ISO }\end{array}$ & & \\
\hline $\begin{array}{l}\text { SA-V -834 (IA-701-A-33) } \\
\text { SA TO A FARH ISO }\end{array}$ & & & $\begin{array}{l}\text { IA-Y } 801 \text { (IA-701-A-11) } \\
\text { IA FLT } 815 \text { OUTLET ISO }\end{array}$ & CLOS & 100 \\
\hline $\begin{array}{l}I A-V-836 \text { (IA-701-A-35) } \\
\text { IA CONN ISO }\end{array}$ & CLOSED & & $\begin{array}{l}\text { IA-V-802 (IA-701-A 6) } \\
\text { IA FLT } 814 \text { OUTLET ISO }\end{array}$ & OPE & 60 \\
\hline $\begin{array}{l}I A-V-833 \text { (GV }-701-A-6) \\
I A \text { PI } 821 \text { I } 50 \text { VLV }\end{array}$ & OPEY & 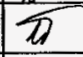 & $\begin{array}{l}\text { IA-V-804 (IA-70I-A-5) } \\
\text { IA FLT } 814 \text { DRN ISO }\end{array}$ & OPEN & \\
\hline $\begin{array}{l}\text { IA-V } 832 \text { (IA-701 A-29) } \\
\text { IA PCV } 802 \text { BY-PASS }\end{array}$ & CLOSED & 4 & $\begin{array}{l}\text { IA-V } 818 \text { (IA-701V-18) } \\
\text { IA FLT 816 ORN ISO }\end{array}$ & OPEN & \\
\hline $\begin{array}{l}\text { IA-V }-827 \text { (IA-701-A-25) } \\
\text { IA FI } 801 \text { INLET ISO }\end{array}$ & OPEN & $\mu$ & $\begin{array}{l}\text { IA-Y-826 (IA-701-A-T4) } \\
\text { IA DRY OUTLET ISO }\end{array}$ & CLOS: & \\
\hline $\begin{array}{l}\text { IA-V-828 (IA-701-8-26) } \\
\text { IAFI } 801 \text { OUTLET ISO }\end{array}$ & & 2 & $\begin{array}{l}\text { IA-V-811 (IA-701 /A } 20) \\
\text { IA FLT 817 INLEI ISO }\end{array}$ & CLOSE & \\
\hline $\begin{array}{l}\text { IA-Y } 829 \text { (IA-701-A-27) } \\
\text { IA FI } 801 \text { BY PASS }\end{array}$ & CLOSED & & $\begin{array}{l}\text { IA-V-813 (IA-701-A 24) } \\
\text { IF FLT } 817 \text { OUTLET ISO }\end{array}$ & CloSE & $\pi$ \\
\hline $\begin{array}{l}\text { IA-V } 830 \text { (IA-701-A-30) } \\
\text { IA PCV } 802 \text { INLET ISO) }\end{array}$ & OPEN & & $\begin{array}{l}\text { IA-Y } 809 \text { (IA- } 701-A=13) \\
I A \text { DRY INLET ISO }\end{array}$ & CLOSE & \\
\hline $\begin{array}{l}\text { IA-V-831 (IA-701-A-31) } \\
\text { IA PCV } 802 \text { OUTLET ISO }\end{array}$ & PPEN & $y$ & $\begin{array}{l}\text { IA-Y } 803 \text { (DR-701-A-10) } \\
\text { IA FLT } 815 \text { DRN ISO }\end{array}$ & OPEN & \\
\hline $\begin{array}{l}\text { IA-V-835 (IA-701-A-32) } \\
\text { IA CONN ISO }\end{array}$ & CLOSED & & $\begin{array}{l}\text { IA-V-819 (IA-70I-A } 23) \\
I A \text { FLT } 817 \text { DRN ISO }\end{array}$ & OPEN, & \\
\hline $\begin{array}{l}\text { IA-V-844 (IA }-701-A-34 \text { ) } \\
\text { IA TO A FARH ISO }\end{array}$ & & & $\begin{array}{l}\text { TA-V-10 (OUTSIDE) } \\
\text { TEMPORARY VALVING }\end{array}$ & OPEN & \\
\hline $\begin{array}{r}\text { * IA- }-840 \text { (IA-701 } \\
\text { BLOG } 702 \text { CROSS T }\end{array}$ & & & & & \\
\hline
\end{tabular}

* Interim air system

** Gate valve, normal flow is straight through. Direction must match direction of valve IA-V-845 (IA-702-15) in CHECKLIST 4. 
HNF-SD-WM-OTR-231, Rev 0

Page 67 of 165

\section{CHECKLIST 3 - INSTRUUMENT AIR VALVING}

\begin{tabular}{|c|c|c|c|c|c|}
\hline VALVE & POSIT & $\mathrm{T}$ & VALVE & POSI & \\
\hline $\begin{array}{l}\text { IA-V-837 (IA-70I A } 38 \text { ) } \\
\text { IA TO AY AZ FARM } 150 \text {. }\end{array}$ & CLOSED & & $\begin{array}{l}\text { SA-Y-854 (AA-701-A.2) } \\
\text { FLT-814 NLET 1SO }\end{array}$ & OPEN & \\
\hline $\begin{array}{l}\left.\text { IA }=V-841 \text { (GV }-701{ }^{-8}-8\right) \\
\text { IA } P I \quad 822 \text { ISO }\end{array}$ & OPEN & $\boldsymbol{n}$ & $\begin{array}{l}S A-Y-855 \text { (IA-701-A-7) } \\
F L T=815 \text { INLET ISO }\end{array}$ & CLOSED & \\
\hline $\begin{array}{l}\left.\star_{\text {SA V }} 860 \text { (IA } 701-\mathrm{A}^{-36}\right) \\
\text { SA FLT SYS BYP VLV }\end{array}$ & CLOSED & & $\begin{array}{l}I A-Y-820 \text { (IA-701-A } 12) \\
\text { IA DRY BYPASS VUV }\end{array}$ & OPEN & \\
\hline $\begin{array}{l}\text { IA-V-838 (IA-701 } A-37) \\
\text { IA I0 241 A ISO }\end{array}$ & CLOSED & $\sim$ & $\begin{array}{l}\text { IA-V-810 (IA-70INA I5) } \\
\text { IA FLT B16 INLET ISO }\end{array}$ & OPEN & \\
\hline $\begin{array}{l}I A-V-842 \text { (GV } 701-A-7) \\
I A \text { PI } 823 \text { ISO }\end{array}$ & OPEN & & $\begin{array}{l}\text { IA-V } 812 \text { (1A-701-A } 19) \\
\text { IA FLT 816 OUTLET ISO }\end{array}$ & TOPE & \\
\hline $\begin{array}{l}S A-V-834 \text { (IA } 701-A-33) \\
\text { SA TO A FARM ISO }\end{array}$ & OPEN & & $\begin{array}{l}\text { IA-Y-801 (IA-701-A IN) } \\
\text { IA FLT 815 OUTLET ISO) }\end{array}$ & CLOSED & \\
\hline $\begin{array}{l}\text { IA-V } 836 \text { (IA-701-A-35) } \\
\text { IA CONN ISO }\end{array}$ & CLOSED & & $\begin{array}{l}\text { IA-V } 802 \text { (IA-701 A-6) } \\
\text { IA FLT } 814 \text { OUTLET ISO }\end{array}$ & DPEN & \\
\hline $\begin{array}{l}\text { IA-V-833 (GV }-701-A-6) \\
\text { IA PI 82.1 ISO VLV }\end{array}$ & OPEN & & $\begin{array}{l}I A-Y-804 \text { (IA-701-A-5) } \\
I A \text { FLT } 814 \text { DRN ISO }\end{array}$ & OPEN & \\
\hline $\begin{array}{l}\text { IA-V } 832 \text { (IA-701-A-29) } \\
\text { IA PCV } 802 \text { BY PASS }\end{array}$ & CLOSED & n & $\begin{array}{l}I A-V-818 \text { (IA-701-A } 18) \\
I A \text { FLT } 816 \text { DRN ISO }\end{array}$ & OPEN & \\
\hline $\begin{array}{l}I A-V-827 \text { (IA-701 } A-25) \\
I A \text { F }\end{array}$ & OPEN & & $\begin{array}{l}\text { IA-Y }-826 \text { (IA-701-A-14) } \\
\text { IA DRY OUTLET ISO }\end{array}$ & CLOS: & \\
\hline $\begin{array}{l}\text { IA-V }-828 \text { (IA-701 A-26) } \\
\text { IA FI } 801 \text { OUTLET ISO }\end{array}$ & OPEN & & $\begin{array}{l}\text { IA-V-811 (IA-701 A-20) } \\
\text { IA FLT 817 INLET ISO, }\end{array}$ & CLOSED & \\
\hline $\begin{array}{l}\text { IA-Y }-829 \text { (IA-701-A }-27) \\
\text { IA FI } 801 \text { BY PASS }\end{array}$ & CLOSED & $\simeq$ & $\begin{array}{l}\text { IA-V-813 (IA-701-AI-24) } \\
\text { IF FLT } 817 \text { OUTLET ISO }\end{array}$ & CLOSED & \\
\hline $\begin{array}{l}\text { IA-V-830 (IA-701-A } 30 \text { ) } \\
\text { IA PCV } 802 \text { INLET ISO }\end{array}$ & OPEN & $=$ & $\begin{array}{l}\text { IA-Y-809, (IA-701-A-13) } \\
\text { IA DRY INLET ISO }\end{array}$ & CLOSED & \\
\hline $\begin{array}{l}\text { IA-V-831 (IA-701-A-31) } \\
\text { IA PCV } 802 \text { OUTLET ISO }\end{array}$ & OPEN & Den & $\begin{array}{l}I A-V-803(D R-701-A-10) \\
\text { IA FLT } 815 \text { DRN ISO }\end{array}$ & OPEN & \\
\hline $\begin{array}{l}\text { IA-V }-835 \text { (IA-701-A-32) } \\
\text { IA CONN ISO }\end{array}$ & CLOSED & & $\begin{array}{l}\text { IA-Y-819 (IA-701-A-23) } \\
\text { IA FLT } 817 \text { DRN ISO }\end{array}$ & OPEN & \\
\hline $\begin{array}{l}\text { IA-V-844 (IA-701-A-34) } \\
\text { IA TO A FARM ISO }\end{array}$ & OPEN & $1 n$ & $\begin{array}{l}\text { TA-V } 10 \text { (OUTSIDE) } \\
\text { TEMPORARY VALVING }\end{array}$ & & \\
\hline $\begin{array}{l}\text { *7 IA V } 840 \text { (IA-7D1-A } 28 \\
\text { BLDG } 702 \text { CROSS TIE VL }\end{array}$ & & - & & & \\
\hline
\end{tabular}

* Interim air system

** Gate valve, normal flow is straight through. Direction must match direction of valve IA-V-845 (IA-702-I5) in CHECKLIST 4. 


\section{CHECKLIST 3 - INSTRUMENT AIR VALVING}

\begin{tabular}{|c|c|c|c|c|c|}
\hline VALVE & POSIIION & INII & VALVE & POS & \\
\hline IA-V-837 (IA-701-A -36$)$ & CLOSED & M/4 & $\begin{array}{l}\text { SAY Y 854 ( }(\mathrm{AA}-701-\mathrm{A}-2) \\
\mathrm{FLT}-814 \text { INET ISO }\end{array}$ & OPEN & \\
\hline IA-V $-841(G V-701-A-8)$ & OPEN & pest & $\begin{array}{l}\text { SA-V } \\
\text { FLT-815 INLET ISO }\end{array}$ & CLOSED & \\
\hline $\begin{array}{l}1 A \text { PI } 822 \text { 1S0 } \\
\text { * SA-Y -860 (IA-701-A-36) } \\
\text { SA FLT SYS BYP VLV }\end{array}$ & CLOSED & & $\begin{array}{l}\text { IA V-B20 (IA-701-A-12) } \\
\text { IA DRY BYPASS VLV }\end{array}$ & OPEN & \\
\hline $\begin{array}{l}1 A-V-838 \text { (IA-701 }-A-37) \\
1 A-T 0241 \text { A ISO }\end{array}$ & CLOSED & & $\begin{array}{l}\text { IA-V }-810 \text { (IA-701-A-15) } \\
\text { IA FLT } 816 \text { INLET ISO }\end{array}$ & OPEN & \\
\hline$(1 A-V-842(6 V-701-A-7)$ & OPEN & & $\begin{array}{l}\text { IA-V } 812 \text { (IA-701 - } 1 \text { II) } \\
\text { IA FLT } 816 \text { OUTLET ISO }\end{array}$ & OPEN & \\
\hline$\frac{1 A}{S A-V-834}(I A-701-A-33)$ & OPEN & & $\begin{array}{l}\text { IA-V-801 (IA-701-A-11) } \\
\text { IA FLT } 815 \text { OUTLET ISO }\end{array}$ & CLOSED & \\
\hline$I_{\mathrm{IA}-\mathrm{V}-836(1 \mathrm{C}-\mathrm{N}-701-\mathrm{A}-35)}$ & CLOSED & & $\begin{array}{l}\text { IA-V } 802 \text { (IA-701-A-6) } \\
\text { IA FLT } 814 \text { OUTLET ISO }\end{array}$ & OPEN & \\
\hline $\begin{array}{l}\text { IA-V-833 (GV }(G 01-A-6) \\
I A \text { PI } 821 \text { I } 50 \text { VLV }\end{array}$ & OPEN & & $\begin{array}{l}\text { IA-V }-804 \text { (IA-701-A-5) } \\
\text { IA FLT } 814 \text { DRN ISO }\end{array}$ & OPEN & \\
\hline $\begin{array}{l}1 A-V-832 \text { (IA-701-A-29) } \\
1 A \text { PCV } 802 \text { BY -PASS }\end{array}$ & CLOSED & & $\begin{array}{l}\text { IA-V-818 (IA-701-A-18) } \\
\text { IA FLT } 816 \text { DRM ISO }\end{array}$ & OPEN & \\
\hline $\begin{array}{l}\text { IA-V } 827 \text { (IA-701-A-25) } \\
\text { IA FI } 801 \text { INLET ISO }\end{array}$ & OPEN & & $\begin{array}{l}\text { IA-Y -826 (IA-701-A-14) } \\
\text { IA DRY OUTLET ISO }\end{array}$ & CLLSED & \\
\hline $\begin{array}{l}\text { IA } R \text { IA-V } 828 \text { (IA-701-A-26) } \\
\text { IA FI } 801 \text { OUTLET ISO) }\end{array}$ & OPEN & & $\begin{array}{l}\text { IA-V-811 (IA-701-A-20) } \\
\text { IA FLT } 817 \text { INLET ISO }\end{array}$ & CLOSED & \\
\hline $\begin{array}{l}\text { IA-V } 829 \text { (IA-701-A-27) } \\
\text { IA FI 801 BY PASS }\end{array}$ & CLOSED & HNA & $\begin{array}{l}\text { IA- V } 813 \text { (1A-701-A 24) } \\
\text { IF FLT } 817 \text { OUTLET ISO }\end{array}$ & CLOSED & \\
\hline $\begin{array}{l}\text { IA-V } 830 \text { (IA-701-A-30) } \\
\text { A PCV } 802 \text { INLET ISO }\end{array}$ & OPEN & & $\begin{array}{l}\text { IA-V }-809 \text { (IA-701-A-13) } \\
\text { IA DRY INLET ISO }\end{array}$ & CLOSED & \\
\hline $\begin{array}{l}\text { IA-V-831 (IA-701 }-A-31 \text { ) } \\
\text { IA PCV } 802 \text { OUTLET ISO }\end{array}$ & OPEN & & $\begin{array}{l}\text { IA-V }-803 \text { (DR-701-A } 10 \text { ) } \\
\text { IA FLT } 815 \text { DRN 150. }\end{array}$ & & \\
\hline $\begin{array}{l}1 A-V-835 \text { (IA-701-A-32) } \\
1 A \text { CONN ISO }\end{array}$ & CLOSED & $\mathrm{X} A \mathrm{~A}$ & $\begin{array}{l}\text { IA-V }-819 \text { (IA-701-A-23) } \\
\text { IA FLT } 817 \text { DRN ISO }\end{array}$ & & \\
\hline $\begin{array}{l}\text { IA-V }-844 \text { (IA } 701-A-34) \\
\text { IA TO A FARA ISO }\end{array}$ & OPEN & hut & $\begin{array}{l}\text { A-V-10 (OUTSIDE) } \\
\text { TEMPORARY VALVING }\end{array}$ & TPE & \\
\hline $\begin{array}{l}* * \text { IA-V } 840 \text { (IA-70I-A-28) } \\
\text { BLDG } 702 \text { CROSS TIE VLY }\end{array}$ & $B$ G A B & & & & \\
\hline
\end{tabular}

* Interim air system

** Gate valve, normal flow is straight through. Direction must match direction of valve IA-V-845 (IA-702-15) in CHECKLIST 4. 


\section{CHECKLIST 3 - INSTRUMENT AIR VALVING}

\begin{tabular}{|c|c|c|c|c|c|}
\hline VALVE & POSITION & INII & VALVE & $\mathrm{PO}$ & \\
\hline $\begin{array}{l}\text { IA V-837 (IA-701 A } 38) \\
\text { IA TO AY AZ FARH ISO }\end{array}$ & CLOSED & & $\begin{array}{l}\text { SA-V-854 (IA-701-A-2) } \\
\text { FLT-814 INLET ISO }\end{array}$ & PPEN & \\
\hline IA-V -841 (GV $-701-A-8)$ & OPEN & & $\begin{array}{l}\text { SA-V-855 (IA-701-A-7) } \\
F_{T-815} \text { INET ISO }\end{array}$ & CLOSED & \\
\hline $\begin{array}{ll}\text { PA I } 822 & \text { ISO } \\
\pi / S A-Y-860 & (1 A-701-A-36)\end{array}$ & CLOSED & & $1 A-V-820(I A-701-A-12)$ & PPEM & \\
\hline SA FLT SYS BYP VLV & andons & & İ DRY BYPASS VLV & & \\
\hline $\begin{array}{l}\text { IA-V-838 (IA-701-A-37) } \\
\text { IA TO } 241 \text { A ISO. }\end{array}$ & CLOSED & & $\begin{array}{l}\text { IA-V-810 (IA-701-A-15) } \\
\text { IA FLT 816 INLET ISO }\end{array}$ & OPEN & \\
\hline $1 A-V-842(G V-701-A-7)$ & OPEN & & $\begin{array}{l}\text { IA-V-812 (IA-701-A-19) } \\
\text { IA FLT } 816 \text { OUTLET ISO }\end{array}$ & OPEN & \\
\hline$\frac{\text { IA PI } 823 \text { ISO }}{\text { SA-V }-834(\text { IA-701-A-33) }}$ & OPEN & & IA-V-801 (IA-701-A-II) & CIOSED & \\
\hline SA TO A FARM ISO & & & IA FLT 815 OUTLEI 150 & & \\
\hline $\begin{array}{l}I A-V-B 36 \text { (IA-701-A-35) } \\
\text { IA CONN ISO }\end{array}$ & CLOSED & & $\begin{array}{l}\text { IA-V-802 (IA-701-A-6) } \\
\text { IA FLT } 814 \text { OUTLET, ISO }\end{array}$ & OPEN & \\
\hline $\begin{array}{l}\text { IA-V-833 (GV-701-A-6) } \\
\text { IA PI } 821 \text { ISO VLV }\end{array}$ & OPEN & & $\begin{array}{l}\text { IA-V-804 (IA-701)A-5) } \\
\text { IA FLT } 814 \text { DRN ISO }\end{array}$ & OPEN & \\
\hline $\begin{array}{l}\text { IA-V-832 (IA-7011-A-29) } \\
\text { IA PCV } 802 \text { BY-PASS }\end{array}$ & CLOSED & & $\begin{array}{l}\text { IA-V-818 (IA-701-A } 18) \\
\text { IA FLT } 816 \text { DRN ISO }\end{array}$ & OPEN & \\
\hline $\begin{array}{l}A \text { IA-Y } 827 \text { (IA-701 A } 25) \\
\text { A F F } 801 \text { INLET ISO }\end{array}$ & OPEN & & $\begin{array}{l}\text { IA-V-826 (IA-701-A-14) } \\
\text { IA DRY OUTLET ISO }\end{array}$ & CEOSE & \\
\hline $\begin{array}{l}\text { IA T I } \\
\text { IA-Y } 828 \text { (IA-701-A-26) } \\
\text { IA FI } 801 \text { OUTLET ISO) }\end{array}$ & OPEN & & $\begin{array}{l}\text { IA-V-811 (IA-701 }-A^{-20} \text { ) } \\
\text { IA FLT } 817 \text { INLET ISO }\end{array}$ & CLOSED & \\
\hline $\begin{array}{l}\text { IA-V-829 (IA-701-A-27) } \\
\text { IA FI } 801 \text { BY PASS }\end{array}$ & CLOSED & & $\begin{array}{l}\text { IA-V-813 (IA-701/ A } 24) \\
\text { IF FLT } 817 \text { OUTLET ISO }\end{array}$ & CLOSE & \\
\hline$\left(\begin{array}{l}\text { A } \\
\text { IA-V }-830 \text { (IA-701-A }-30) \\
\text { IA.PCV } 802 \text { INLET/ISO }\end{array}\right.$ & OPEN & & $\begin{array}{l}\text { IA-Y-809 (IA-701-A-13) } \\
\text { IA DRY INLET ISO }\end{array}$ & CLOSED & \\
\hline 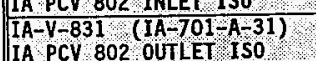 & OPEN & & $\begin{array}{l}\text { IA-V }-803(\mathrm{DR}-701-\mathrm{A}-10) \\
\text { IA FLT } 815 \text { DRN ISO }\end{array}$ & OPEN & \\
\hline $\begin{array}{l}\text { IA-V }-835 \text { (IA-701-A-32) } \\
\text { IA CONN ISO }\end{array}$ & CLOSED & & $\begin{array}{l}\text { IA-Y-819 (IA-70I-A-23) } \\
\text { IA FLT } 817 \text { DRN ISO }\end{array}$ & & \\
\hline $\begin{array}{l}\text { IA CONAN } \\
\text { IA-V-844 (IA-701-A-34) } \\
\text { IA TO A FARM 150 }\end{array}$ & PPEN & & $\begin{array}{l}\text { TA-V } 10 \text { (OUTSIDE) } \\
\text { TEMPORARY VALVING }\end{array}$ & PPEN & \\
\hline${ }^{*} * 1 \mathrm{~A}-\mathrm{V}-840(\mathrm{IA-701- \textrm {A } - 2 8}$ & 38 & & & & \\
\hline
\end{tabular}

* Interim air system

** Gate valve, normal flow is straight through. Direction must match direction of valve IA-V-845 (IA-702-15) in CHECKLIST 4. 


\section{CHECKLIST 4 - ACCUMULATOR INSTRUMENT AIR VALVINGG}

NOTE - Flow through valve IA-V-845 (IA-702-15) MUST match flow through valve IA-V-840 (IA-701-A-28) in table 3 . When direction $C$ or $A$ is chosen for one valve it must also be chosen for the second.

\begin{tabular}{|c|c|c|c|c|c|}
\hline VALYE & POSI & IIT & VALVE & Pos & \\
\hline $\begin{array}{l}A-Y-862 \\
A 150 \text { VLV }\end{array}$ & $\mathrm{A}^{\mathrm{B}}$ & T & 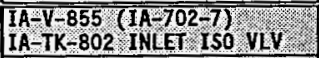 & OPEN & \\
\hline $\begin{array}{l}\mathrm{V}-863 \\
\mathrm{Is0} \text { VLV }\end{array}$ & $A^{2} B$ & TK & $\begin{array}{l}\text { IA-V } 854 \text { (IA } 702 \text { (8) } \\
\text { IA-TK-802 BYP YLV }\end{array}$ & CLOSED & \\
\hline To A FARM & $A=B$ & no & $\begin{array}{l}-V-857 \text { (IA } 702-10) \\
-T K-802 \text { OUTLET ISO VLV }\end{array}$ & KOSED & \\
\hline $\begin{array}{l}846 \text { (1A-702-1) } \\
\text { A FARM } 150\end{array}$ & & $\pi<$ & $\begin{array}{l}-859 A \text { (IA-702-11) } \\
\text { PCV- } 803 \text { A INLET I } 50\end{array}$ & OPEN & \\
\hline$-847\left(1 A^{-702 \% 2)}\right.$ & CLOSED & $\pi^{c}$ & $\begin{array}{l}\text { V } 8598 \text { (TA-702 } 13 \text { ) } \\
\text { PCV-803B INLET ISO }\end{array}$ & $\mathrm{CL}$ & \\
\hline $\begin{array}{l}\text { (IA-702-3) } \\
\text { FARM ISO }\end{array}$ & OPEN & $\pi k$ & 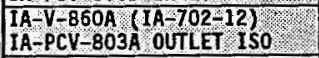 & OPEN & \\
\hline $\begin{array}{l}850 \text { (IA-702-4) } \\
\text { M INST AIR TEST CONN }\end{array}$ & CLOSED & $T h$ & 14) 150 & CLOSED & \\
\hline$\frac{1851}{V}(1 A-702-5)$ & CLOSED & 5 & $02-9)$ V & & \\
\hline 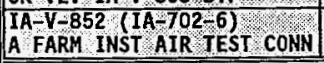 & CLOSED & & 1. & & \\
\hline \multicolumn{6}{|c|}{$\begin{array}{l}\text { THE FOUR VALVES LISTED BELOW ARE LOCATED INSIDE THE } 702-A \text { BUILOING AND ARE THE } \\
\text { RESPONSIBILITY OF EF-1 (PROCESS OPERATIONS) }\end{array}$} \\
\hline $4(I A-702-18)$ & $\overline{A-B}$ & 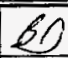 & $\begin{array}{l}\text { IA-V }-865 \text { (IA-TO2-19) } \\
\text { IA TO VENT SYS }\end{array}$ & $A-B$ & \\
\hline $1 \mathrm{~A}-702-20$ (Nö H-14H) & $B-C$ & $\beta_{1}$ & $1 A-702-21$ (No & & \\
\hline-702 & & & & & \\
\hline
\end{tabular}

* Three-way valve, normal flow path is with valve handle PARALLEL to pipe run.

** Three-way vaive, normal flow path is with valve handle PERPENDICULAR to pipe run.

*** Three-way valve, normal flow path is with valve handle PERPENDICULAR to pipe run. Direction must match direction of valve IA-V-840 (IA-701-A-28) in CHECKLIST 3. 


\section{CHECKLIST 4 - ACCUMULÁTOR INSTRUMENT AIR VALVING}

NOTE - Flow through valve IA-V-845 (IA-702-15) MUST match flow through valve IA-V-840 (1A-701-A-2B) in table 3 . When direction $C$ or $A$ is chosen for one valve it must also be chosen for the second.

\begin{tabular}{|c|c|c|c|c|c|}
\hline VALVE & POSITION & & VALVE & POSITI & \\
\hline $\begin{array}{l}\text { IA Y Y } 862 \text { (IA } \\
\text { IA ISO V V }\end{array}$ & A-B. & & $\begin{array}{l}\text { IA-V } 855 \text { (1A-702-7) } \\
\text { IA-TK-802 INLET ISO VLV }\end{array}$ & OPEN & \\
\hline $\begin{array}{l}\text { IA-Y }-863 \\
\text { IA ISO VLY }\end{array}$ & A-B & & 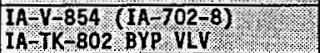 & CLOSED & \\
\hline $\begin{array}{l}\text { IA-V }-845 \text { (IA } \rightarrow 02-15) \\
\text { IA TO A FARM }\end{array}$ & $A-B$ & & $\begin{array}{l}\text { IA-V-857 (IA-702 10) } \\
\text { IA-TK-802 OUTLET ISO VLV }\end{array}$ & CLOSED & \\
\hline $\begin{array}{l}\text { IA }-V-846 \text { (IA } 702-1) \\
\text { IA TO A FARM ISO }\end{array}$ & OPEN & & $\begin{array}{l}\mathrm{IA}-\mathrm{Y} / 859 \mathrm{~A} \text { (1A-702 11) } \\
\text { IA-PCV-803A INLE ISO }\end{array}$ & OPEN & \\
\hline $\begin{array}{l}\bar{I} A-Y-847 \text { (IA } 702-2) \\
\text { A FARI INST AIR TEST CONN }\end{array}$ & CLOSED & & $\begin{array}{l}\text { IA-V-859B (IA-702-13) } \\
\text { IA-PCV-803B INLET ISO }\end{array}$ & CLOSED & \\
\hline $\begin{array}{l}\text { IA-V-849 (1A-702-3) } \\
\text { IA TO A FARM ISO }\end{array}$ & OPEN & & $\begin{array}{l}\text { IA- } 1860 \mathrm{~A} \text { (IA-702-12) } \\
\text { IA-PCV-803A OUTLET ISO }\end{array}$ & OPEN & \\
\hline $\begin{array}{l}\text { IA-V-850 }(1 \mathrm{~A}-702-4) \\
\text { A FARH INST AIR TEST CONN }\end{array}$ & CLOSED & & $\begin{array}{l}\mathrm{IA}-\mathrm{V}-860 \mathrm{~B} \text { (IA-702-14) } \\
\mathrm{IA-PCV}-803 \mathrm{~B} \text { OUTLET ISO }\end{array}$ & CLOSED & \\
\hline $\begin{array}{l}\text { IA-V } 851 \text { ( }(1 A-702-5) \\
C K \text { VLV IA-V }-853 \text { BYP }\end{array}$ & CLOSED & & $\begin{array}{l}\text { IA-V }=856 \text { (IA-702-9) } \\
\text { IA-TK-802 DRN ISO VIV }\end{array}$ & $\mathrm{CE}$ & \\
\hline $\begin{array}{l}\text { IA-V }-852 \text { (IA-702-6) } \\
\text { A FARM INST AIR TEST CONN }\end{array}$ & CLOSED & & & & \\
\hline \multicolumn{6}{|c|}{$\begin{array}{l}\text { THE FOUR VALYES LISTED BELOH ARE LOCATED INSIDE THE 702-A BUILDING AND ARE THE } \\
\text { RESPONSBBILITY OF EF-1 (PROCESS OPERATIONS) }\end{array}$} \\
\hline $\begin{array}{l}\text { IA-Y-864 (IA-702-18) } \\
\text { IA TO VENT SYS }\end{array}$ & & जो & $\begin{array}{l}\text { IA-V }-865 \text { (IA-702-19) } \\
\text { IA TO VENT SYS }\end{array}$ & $A^{B}, 1$, & \\
\hline $\mathrm{IA}-702-20(\mathrm{No} H-14 \#)$ & $B-C$ & & $\mathrm{IA}-702-21$ (No H-14 (f) & $B-C$ & \\
\hline $\mathrm{IA}-702-22\left(\mathrm{No} \mathrm{H}^{-144}\right)$ & $B-C$ & & & 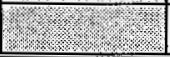 & \\
\hline
\end{tabular}

* Thiree-way valve, normal flow path is with valve handle PARALLEL to pipe run.

** Three-way valve, normal flow path is with valve handle PERPENDICULAR to pipe run.

*** Three-way valve, normal flow path is with valve handle PERPENDICULAR to pipe run. Direction must match direction of valve IA-V-840 (IA-701-A-28) in CHECKLIST 3. 


\section{CHECKLIST 4 - ACCUMULATOR INSTRUMENT AIR VALVING}

NOTE - Flow through valve IA-V-845 (IA-702-15) MUST match flow through valve IA-V-840 (IA-701-A-28) in table 3 . When direction $C$ or $A$ is chosen for one valve it must also be chosen for the second.

\begin{tabular}{|c|c|c|c|c|c|}
\hline VALVE & POSITION & INIT & VALVE & POSIT & \\
\hline $\begin{array}{l}\text { IA-V } 862 \text { (IA } 702-15) \\
\text { IA ISO VLV }\end{array}$ & $A-B$ & 10 & $\begin{array}{l}\text { IA-V-855 (IA-702-7) } \\
\text { IA-TK-802 INLEI ISO VLV }\end{array}$ & DPEN & \\
\hline $\begin{array}{l}\text { IA-V-863 (IA-702-17) } \\
\text { IA IS0 VLV }\end{array}$ & A-B & & $\begin{array}{l}\text { IA-V } 854 \text { (IA-702-8) } \\
\text { IA-TK-802 BYP VLV }\end{array}$ & CLOSED & \\
\hline $\begin{array}{l}\text { IA-V } 845 \text { (IA-702-15) } \\
\text { IA TO A FARM }\end{array}$ & $A-B$ & & $\begin{array}{l}\text { IA-V-857 (1A-702-10) } \\
\text { IA-TK-802 OUTLET ISO VLV }\end{array}$ & CLOSED & \\
\hline $\begin{array}{l}\text { IA-V-846 (IA-702-1) } \\
\text { IA TO A FARM ISO }\end{array}$ & & & $\begin{array}{l}\text { IA-V-859A (IA-702-II) } \\
\text { IA-PCV-803A INLET I SO }\end{array}$ & OPEN & \\
\hline $\begin{array}{l}\text { IA-V }-847 \text { (IA-702-2) } \\
\text { A FARM INST AIR TEST CONN }\end{array}$ & CLOSED & & $\begin{array}{l}\text { IA-V } 859 B \text { (IA-702-13) } \\
\text { IA-PCV-803B INLET ISO }\end{array}$ & CLOSED & \\
\hline $\begin{array}{l}\text { IA-V } 849 \text { (IA-702-3) } \\
\text { IA TO A FARM ISO }\end{array}$ & OPEN & & $\begin{array}{l}\mathrm{IA}-\mathrm{V}-860 \mathrm{~A} \text { (IA-702-12) } \\
\mathrm{IA}-\mathrm{PCV}-803 \mathrm{~A} \text { OUTLET ISO }\end{array}$ & OPEN & $y \sqrt{y}$ \\
\hline $\begin{array}{l}\text { IA-V } 850 \text { (IA-702-4) } \\
\text { A FARM INST AIR TEST CONN }\end{array}$ & CLOSED & & $\begin{array}{l}I A-V-860 B \\
I A-P C Y-803 B \text { (IA-702-14) }\end{array}$ & CLOSED & \\
\hline $\begin{array}{l}\text { IA-V } 851 \text { (IA-702-5) } \\
C K \text { VLV IA-V } 853 \text { BYP }\end{array}$ & CLOSED & & $\begin{array}{l}\text { IA-V-856 (IA-702-9) } \\
\text { IA-TK-802 DRN ISO VLV }\end{array}$ & $\mathrm{CLO}$ & \\
\hline $\begin{array}{l}\text { IA-V-852 (IA-702-6) } \\
\text { A FARM INST AIR TEST CONN }\end{array}$ & CLOSED & & & & \\
\hline \multicolumn{6}{|c|}{$\begin{array}{l}\text { THE FOUR VALVES LISTED BELON ARE LOCATED INSIDE THE 702-A BUILDING AND ARE THE } \\
\text { RESPONSIBILITY OF EF-1 (PROCESS OPERATIONS) }\end{array}$} \\
\hline $\begin{array}{l}\text { IA-V-864 (IA-702-18) } \\
\text { IA TO VENT SYS }\end{array}$ & A-B & & $\begin{array}{l}\text { IA-V } 865 \text { (IA }-702-19 \text { ) } \\
\text { IA TO VENT SYS }\end{array}$ & $A-B$ & \\
\hline $\mathrm{IA-702-20}\left(\mathrm{No} \mathrm{H}^{-14} \mathrm{H}\right)$ & $\mathrm{B}-\mathrm{C} / \mathrm{S}$ & & $\mathrm{IA}-702-21(\mathrm{No} \mathrm{H}-14 /)$ & & \\
\hline $1 \mathrm{~A}-702-22\left(\mathrm{No} \mathrm{H}^{-14}\right.$ & $B-C$ & & & & \\
\hline
\end{tabular}

$$
\text { PPA 3-3-97 }
$$

* Three-way valve, normal flow path is with valve handle PARALLEL to pipe run.

** Three-way valve, normal flow path is with valve handle PERPENDICULAR to pipe run.

*** Three-way valve, normal flow path is with valve handle PERPENDICULAR to pipe run. Direction must match direction of valve IA-V-840 (IA-701-A-28) in CHECKLIST 3. 
HNF-SD-WM-OTR-231, Rev 0 Page 73 of 165

\section{CHECKLIST 4 - ACCUMULATOR INSTRUMENT AIR VALVING}

NOTE - Flow through valve IA-V-845 (IA-702-15) MUST match flow through valve IA-V-840 (IA-701-A-28) in table 3 . When direction $C$ or $A$ is chosen for one valve it must also be chosen for the second.

\begin{tabular}{|c|c|c|c|c|c|}
\hline VALVE & POSITION & INIT & VALVE & POSIIION & $\mathbf{I T}$ \\
\hline $\begin{array}{l}I A-V-862 \\
I A-150 \text { V }(I A-702-16)\end{array}$ & $A-B$ & B) & $\begin{array}{l}\text { TA-Y }-855 \text { (IA-702-7) V V } \\
\text { IA-TK-802 INLET ISO VL }\end{array}$ & OPEN & \\
\hline $\begin{array}{l}I A-V-863(I A-702-17) \\
I A \text { ISO V } V^{\prime}\end{array}$ & $A-B$ & & $\begin{array}{l}\text { IA-V-854 (IA 702 }-8) \\
I A-T K-802 \text { BYP } V \text { V }\end{array}$ & CEOSEO & \\
\hline $\begin{array}{l}\left.\text { IA-V-845 (IA- } 702^{-15}\right) * * t \\
\text { IA TO A FARM }\end{array}$ & $A-B$ & Du & $\begin{array}{l}\text { IA-V } \\
\text { IA-TK-857 (IA-702-10) }\end{array}$ & CLOSED & \\
\hline $\begin{array}{l}\text { IA-V } 846 \text { (IA-702-1) } \\
\text { IA TO A FARM ISO }\end{array}$ & & 0 & $\begin{array}{l}\text { IA }-8598 \text { (IA } 702-11) \\
\text { IA-PCV-803A INLET ISO }\end{array}$ & OPEN & \\
\hline $\begin{array}{l}\text { IA-V } 847 \text { (1A-702-2) } \\
\text { A FARM INST AIR TEST CONN }\end{array}$ & CLOSED & & $\begin{array}{l}\text { IA-Y-859B (IA-702313) } \\
I A-P C V-803 B \text { INLET ISO }\end{array}$ & CLOSED & \\
\hline $\begin{array}{l}\text { IA-V-849 (IA-702-3) } \\
\text { IA TO A FARM ISO }\end{array}$ & & & $\begin{array}{l}I A-V-860 \mathrm{~A} \text { (IA-702-12) } \\
I A-P C V-803 A \text { OUTLET ISO }\end{array}$ & OPEN & \\
\hline $\begin{array}{l}\text { IA-V }-850 \text { (IA-702 } 4) \\
\text { A FARM INST AIR TEST CONN }\end{array}$ & CLOSED & & $\begin{array}{l}\text { IA-V-860B (IA-702-14) } \\
I A-P C V-803 B \text { OUTLET ISO }\end{array}$ & CLOSED & \\
\hline $\begin{array}{l}\text { IA-V-851 (IA-702-5) } \\
C K \text { VLV IA-V-853 BYP. }\end{array}$ & CLOSED & & $\begin{array}{l}I A-V-856 \text { ( } 1 A-702-9) \\
I A-T K-802 \text { DRN ISO VLV }\end{array}$ & CLOSED & \\
\hline $\begin{array}{l}\text { IA-V } 852 \text { (IA-702-6) } \\
\text { A FARN INST AIR TEST CONN }\end{array}$ & CLOSED & 7 & & & \\
\hline \multicolumn{6}{|c|}{$\begin{array}{l}\text { THE FOUR VALYES LISTED BELOH ARE LOCATED INSIDE THE 702-A BUILDING AND ARE THE } \\
\text { RESPONSIBILITY OF EF-1. (PROCESS OPERATIONS) }\end{array}$} \\
\hline $\begin{array}{l}\text { IA-V-864 }(I A-702-18) \\
\text { IA TO VERT SYS }\end{array}$ & $A-B$ & F) & $\begin{array}{l}\text { IA Y Y-865 ( }(1 A-702-19) \\
\text { IA TO VENT SYS }\end{array}$ & $\overline{A-B}$ & \\
\hline $\mathrm{IA}-702-20\left(\mathrm{No} H-14 \frac{\pi}{7}\right)$ & $B-C$ & $\$$ & $\mathrm{IA}^{-702-21}(\mathrm{NoH}-14 \%)$ & $B-C$ & \\
\hline 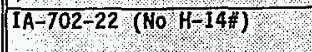 & $B-C$ & & & & \\
\hline
\end{tabular}

* Three-way valve, normal flow path is with valve handle PARALLEL to pipe run.

** Three-way valve, normal flow path is with valve handle PERPENDICULAR to pipe run.

*** Three-way valve, normal flow path is with valve handle PERPENDICULAR to pipe run. Direction must match direction of valve IA-V-840 (IA-701-A-28) in CHECKLIST 3. 


\section{CHECKLIST 5 - ELECTRICAL LINE-UP}

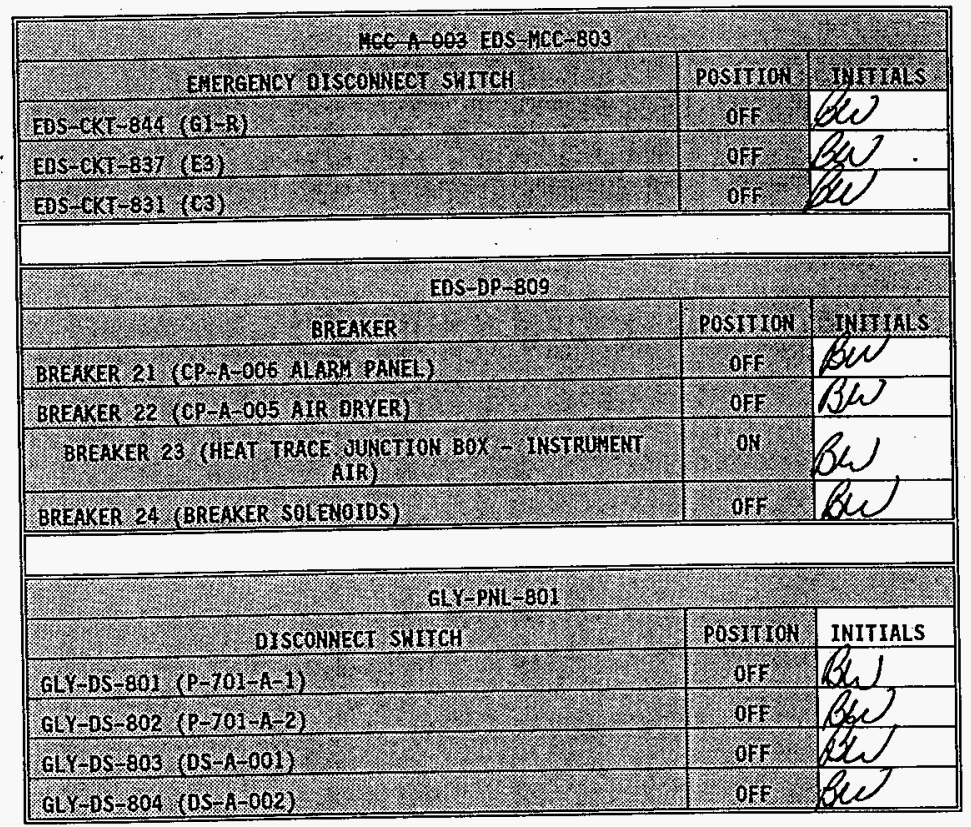




\section{CHECKLIST 5 - ELECTRICAL LINE-UP}

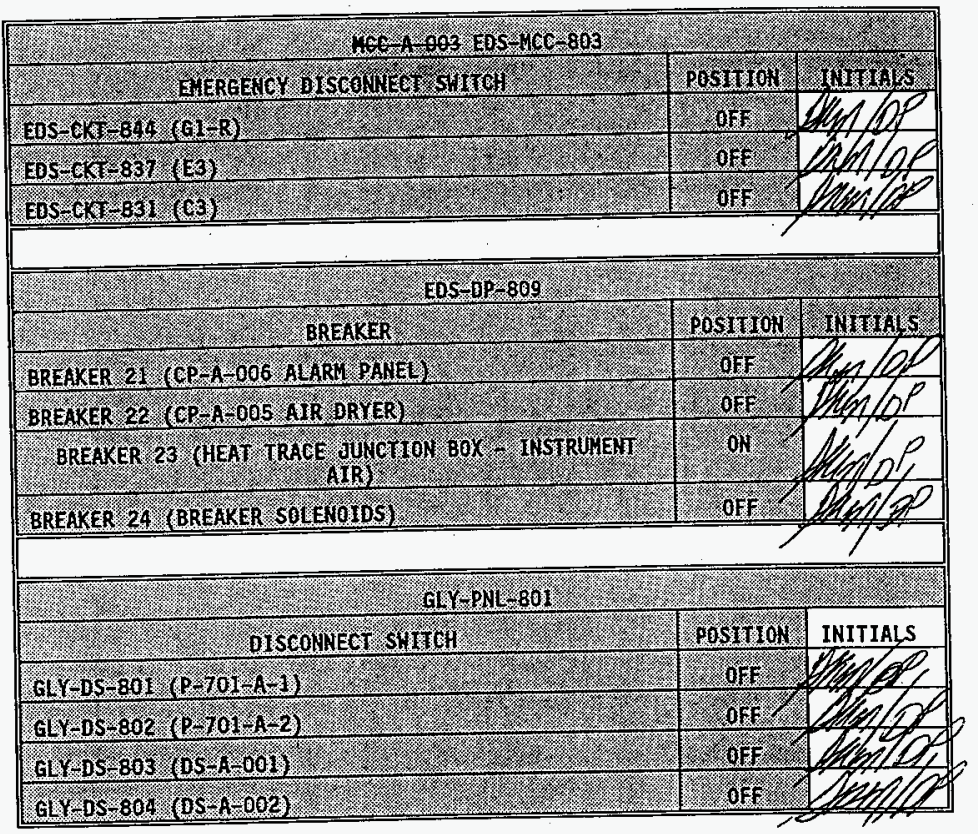


CHECKLIST 5 - ELECTRICAL LINE-UP

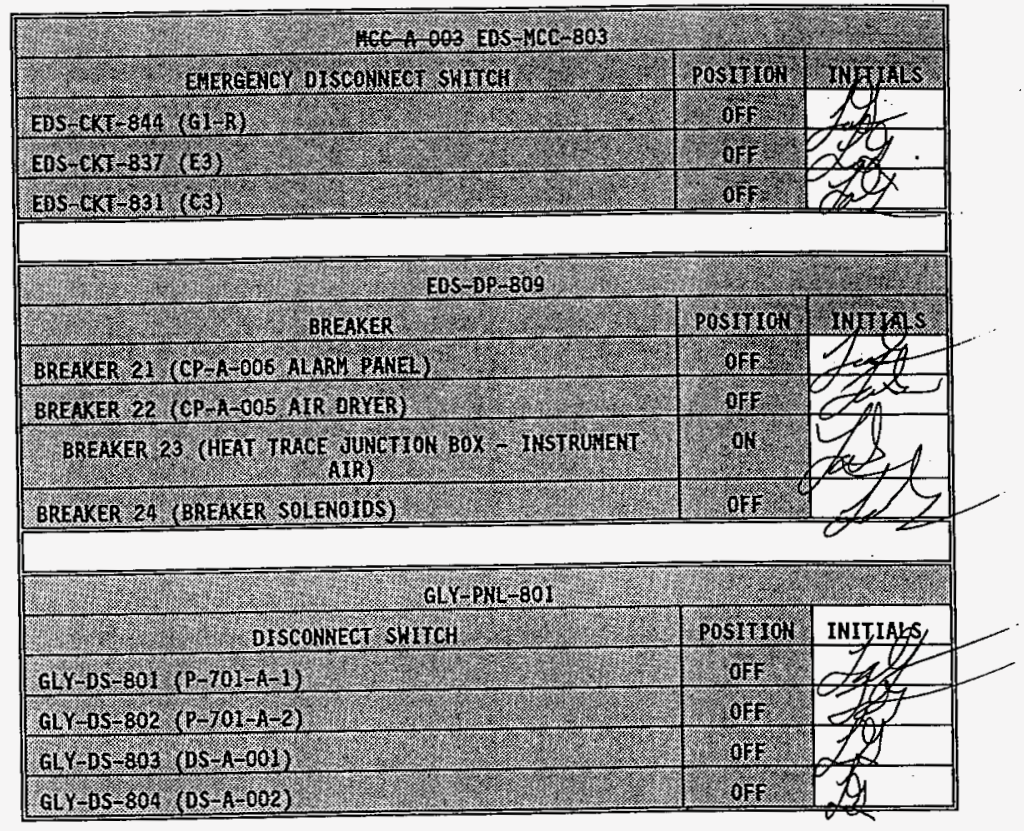




\section{CHECKLIST 5 - ELECTRICAL LINE-UP}

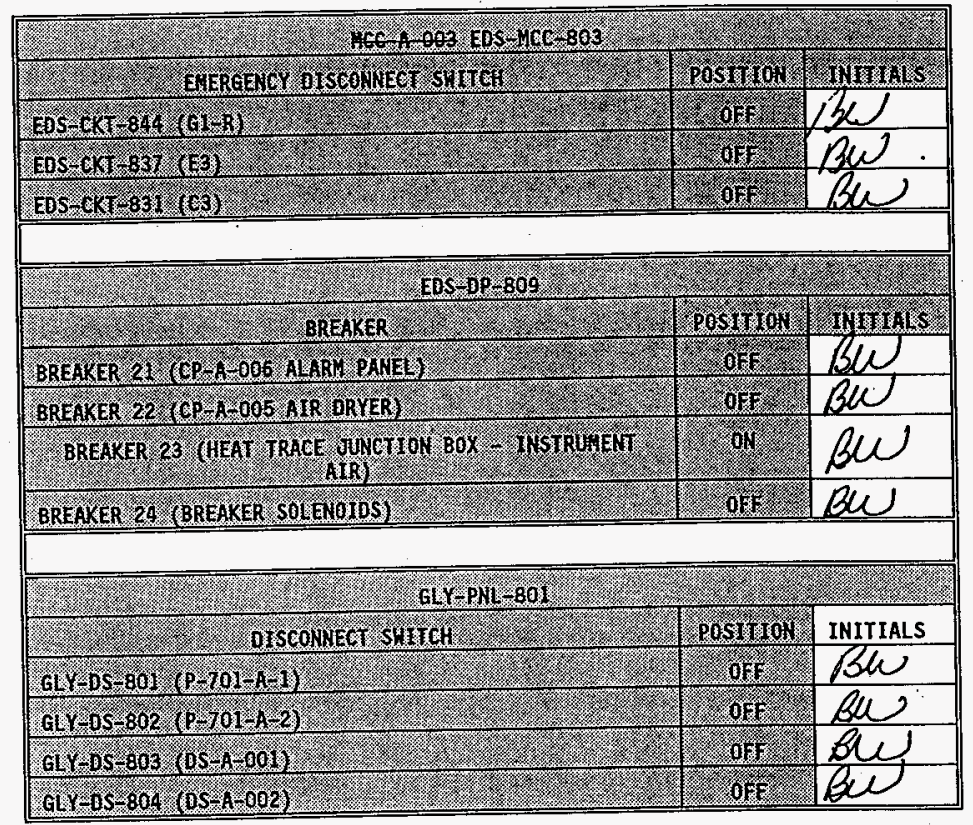


HNF-SD-WM-OTR-231, Rev 0

Page 78 of 165

CHECKLIST 5 - ELECTRICAL LINE-UP

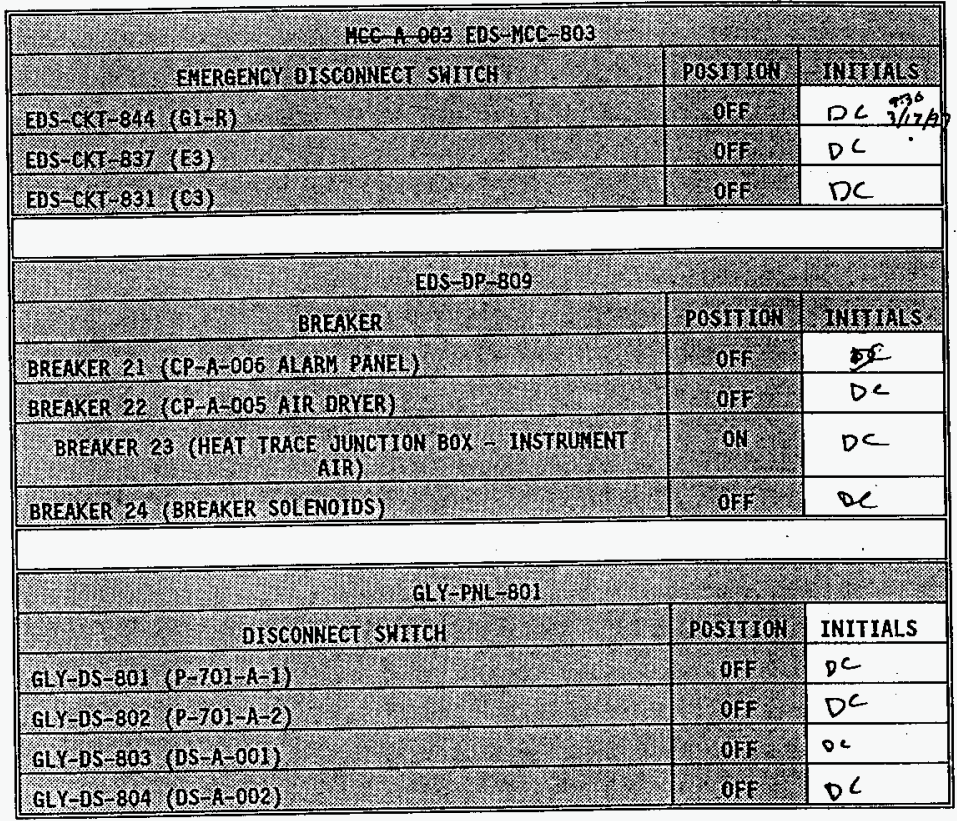


HNF-SD-WM-OTR-231, Rev 0

Page 79 of 165

\section{8-HOUR ON-LINE TEST DÄTA SHEET}

FOR SA-CMP-801

\begin{tabular}{|c|c|c|c|c|c|}
\hline PARAMETER & DATE & TIME & READING & EXPEGTED RANGE & INH \\
\hline 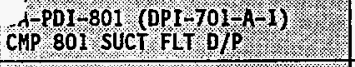 & $-3 / 10 / 9 n$ & 1135 & 2 & 6. PSI & TK \\
\hline 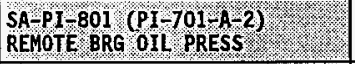 & & & 46 & $15 \cdot 6 \cdot 66.5 s 1$ & $\pi c$ \\
\hline $\begin{array}{l}\text { SAPI-805 (PI-701-A-1) } \\
\text { INTER STG DISCH PRESS }\end{array}$ & & & 26 & $20-40 . \mathrm{PSI}$ & $\pi k$ \\
\hline 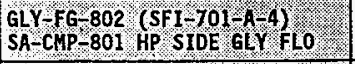 & & & & Flow r , & $T K$ \\
\hline $\begin{array}{l}\text { SA-P1 } 803 \text { ( }(\mathrm{PI}-701-1-4) \\
\text { CMP } 801 \text { DISCH PRESS }\end{array}$ & & & 95 & $85-105$ PSI & Mc \\
\hline $\begin{array}{l}\text { SA-TI-801 (TI-701-A-2) } \\
\text { CHP } 801 \text { SA DISCH TEMP). }\end{array}$ & & & 52 & $90-120 . \mathrm{F}$ & $\pi k$ \\
\hline $\begin{array}{l}\text { SA-SG-801 } \\
\text { SA CMP } 01 \mathrm{LV} \text { LV }\end{array}$ & & & & ABOVE MARK & $\pi$ \\
\hline $\begin{array}{l}\text { 6LY-T I-803 }(\mathrm{TI}-701-\mathrm{A}-7) \\
\text { SA-CMP-801 GLY INLET TEMP }\end{array}$ & & & 72 & $90-120 \%$ & $\pi x$ \\
\hline $\begin{array}{l}\text { GLY-TI-801 (TI-701-A-9) } \\
\text { SA-CMP-801 GLY DISCH TEMP }\end{array}$ & - & & 109 & $90-120.7$ & $T K$ \\
\hline $\begin{array}{l}\text { YFI-801 (FI-701-A-3) } \\
\text { I-CMP-801 SYS GLY FLO IND }\end{array}$ & & & ( ) & K67. GPI & Tz \\
\hline $\begin{array}{l}\text { SA-PISL-801 (PI-70I-A-1) } \\
\text { SA HDR LO PRESS ALART }\end{array}$ & & & 96 & $90=100$ PSI & The \\
\hline $\begin{array}{l}\text { SA-TI-805 (TI-701-A-5) } \\
\text { SERVICE AIR HDR TEMP }\end{array}$ & & & 0 & $90-120 \%$ & Tk \\
\hline $\begin{array}{l}\text { DUTY CYCLE, } \\
\text { (LOAD TIME/TOTAL TIME) }\end{array}$ & 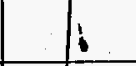 & ${ }^{4}$ & $30 / 136$ & & TA \\
\hline RELATIVE HUMDITY & & & $68 \%$ & (1.: & \\
\hline ATMOSPHERIC PRESSURE & & & 29.36. & & \\
\hline INSIDE TEMP. $(\mathrm{IN} 701 \mathrm{~A})$ & & V & 64 & 1) & $T R$ \\
\hline $\begin{array}{l}\text { OUTSIDE TEMP. } \\
\text { (OUTSIDE } 701-A \text { ) }\end{array}$ & & & $43^{\circ}$ & & \\
\hline
\end{tabular}

* Call weather station for required information at 373-2875

Note: If readings are outside designated ranges, Notify Test Director.

(1) Guage veeds tw be replaced : Gly - Fl-802 Reads 42 


\section{8-HOUR ON-LINE TEST DATA SHEET}

FOR SA-CMP-801

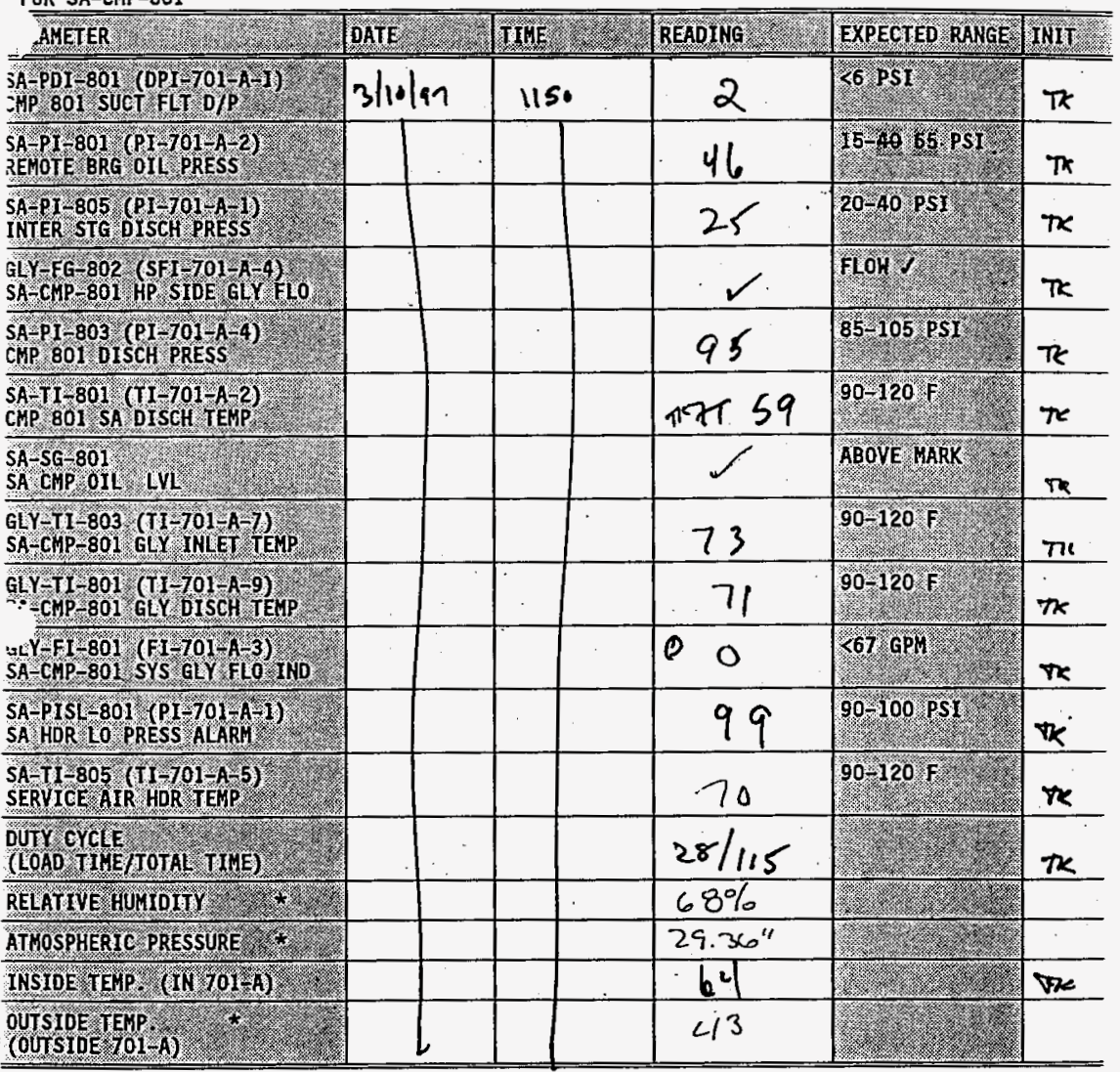

* Call weather station for required information at 373-2875

Note: If readings are outside designated ranges, Notify Test Directọr. 
HNF-SD-WM-OTR-231, Rev 0

Page 81 of 165

\section{8-HOUR ON-LINE TEST DATA SHEET}

\begin{tabular}{|c|c|c|c|c|c|}
\hline PARANETER & DATE & TIME & READING & EXPECTED RANGE & $1 \mathrm{NIT}$ \\
\hline 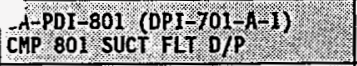 & $3 / 16 \mid a 7$ & 1205 & & $26 . P S I$ & $\pi x$ \\
\hline $\begin{array}{l}\text { SA-PI-801 (PI-701-AI) } \\
\text { REMOTE BRG OIL PRES }\end{array}$ & & & & $15-16 \% 65 \mathrm{ps}$. & $\pi c$ \\
\hline $\begin{array}{l}\text { SAPI-805 (PI-701 A A) } \\
\text { INTER STG OISCH PRESS }\end{array}$ & & & & 20. 40.851 & th \\
\hline $\begin{array}{l}\text { GLY -FG } 802 \text { (SFI-701 A } 4 \text { ) } \\
\text { SA-CMP-801 HP SIDE GLY Flo }\end{array}$ & & & & Flow Y & $\pi$ \\
\hline $\begin{array}{l}\text { SA-PI } 803 \text { (PI-701-A-4) } \\
\text { CMP } 801 \text { DISCH PRESS. }\end{array}$ & & & & 85. $105.9 S 1$ & te \\
\hline $\begin{array}{l}\left.\text { SA-TI-80I (TI }-701-\text { A }^{2} \text { ) }\right) \\
\text { CMP } 801 \text { SA DISCH TEPP) }\end{array}$ & & & & $90-120 \%$ & ne \\
\hline $\begin{array}{l}\text { SA-SG-801 } \\
\text { SA CMP OIL LVL }\end{array}$ & & & & ABOVE MARK & $T x$ \\
\hline $\begin{array}{l}\text { GLY }-T I-803 \text { (TI-701-A } 7 \text { ) } \\
\text { SA-CMP-801 GLY INLET TEMP }\end{array}$ & & & & $90-120 \mathrm{P}$ & the \\
\hline $\begin{array}{l}\text { GLY-TI-801 (TI 70I-A-9) } \\
\text { SA-CHP-801 GLY DISCA TEMP. }\end{array}$ & & & & $90-120 \%$ & TK \\
\hline $\begin{array}{l}\text { TY-FI-801 (FI-701 A-3) } \\
\text { A-CMP-801 SYS GI FLO IND }\end{array}$ & & & $\theta$ & 6.7 GPY & nk \\
\hline $\begin{array}{l}\text { SA-PISL } 801 \text { (PI- TOI A I) } \\
\text { SA HDR LO PRESS ALARH }\end{array}$ & & & & $90-100 \% 951$ & Th \\
\hline $\begin{array}{l}\text { SA-TI-805 (TI-701-A-5) } \\
\text { SERVICE AIR HDR TEPP }\end{array}$ & & & & $90-120.9$ & the \\
\hline $\begin{array}{l}\text { DUTY CYCLE, TOTAL TIME) } \\
\text { (LOAD TIUE/ TO }\end{array}$ & & & $29 / 1 / 6$ & & $T K$ \\
\hline RELAT IVE HUMTDITY & & & $68 \%$ & & \\
\hline ATMOSPHERIC PRESSURE & & & $27.36 " 1$ & & \\
\hline INSIDE TEMP - ( IN $/ 201-A)$ & & & 67 & & Me \\
\hline $\begin{array}{l}\text { OUTSIDE TEMP. } \\
\text { (OUTSIDE 701-A) }\end{array}$ & 木 & & $413^{\circ} p$ & & \\
\hline
\end{tabular}

* Call weather station for required information at $373-2875$

Note: If readings are outside designated ranges, Notify Test Director. 


\section{8-HOUR ON-LINE TEST DATA SHEET}

FOR SA-CMP-801

\begin{tabular}{|c|c|c|c|c|c|}
\hline PARAAETER & DATE. & $\mathrm{THE}$ & READING & EXPECTED RANGE & INIT \\
\hline 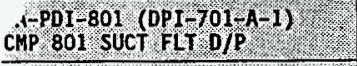 & $3 / 10 / 92$ & 1220 & 2 & 66. PSI & $T K$ \\
\hline $\begin{array}{l}\text { SA-PI-801 (PI 701,A-2) } \\
\text { REMOTE BRG OIL PRESS. }\end{array}$ & & & 46 & 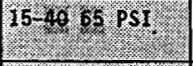 & $7 x$ \\
\hline $\begin{array}{l}\text { SA - PI } 1-805 \text { (PI-701-A-1) } \\
\text { INTER STE DISCH PRESS }\end{array}$ & & & 26 & $20=40.9 S 1$ & $\pi K$ \\
\hline $\begin{array}{l}\text { GLY-FG-802 (SFI-701 A } 4 \text { ) } \\
\text { SA-CHP-801 HP } 510 E \text { G } F \text { L }\end{array}$ & - & & & Flor & $7 c$ \\
\hline $\begin{array}{l}\text { SA-PI-803 (PI-701-A } 4) \\
\text { CHP } 801 \text { DISCH PRESS }\end{array}$ & & & & $85.105 \%$ PI & $\pi$ \\
\hline $\begin{array}{l}\text { SA-TI-801 (TI-101-A-2) } \\
\text { CHP BOI SA DISCH TEMP) }\end{array}$ & & & 6 & $90-120, F$ & $7 x$ \\
\hline $\begin{array}{l}\text { SA-SG-801 } \\
\text { SA CHP OIL LVL. }\end{array}$ & & & & ABOVE MAF & tr \\
\hline $\begin{array}{l}\text { GLY-TI-803 (TI-701-A-7) } \\
\text { SA-CMP-801 GLY INLEI TEMP }\end{array}$ & & & 83 & $90-120 \mathrm{f}$ & $\pi$ \\
\hline $\begin{array}{l}\text { GLY-TI-801 (TI-701-A-9) } \\
\text { SA-CMP-801 GLY DISCH TEFP }\end{array}$ & & & & $90-120$ & Dine \\
\hline $\begin{array}{l}\text { AY-FI-801 (FI-701-A - } 3 \text { ) } \\
\text {-CMP-801 SYS GLY FLO IND }\end{array}$ & & & & K67 GPH & 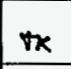 \\
\hline $\begin{array}{l}\text { SA-PISL-BO1 (PI-701-A-I) } \\
\text { SA HDR LO PRESS ALARM }\end{array}$ & & & & $90-100$ PSI & Tr \\
\hline $\begin{array}{l}\text { SA-TI-805 (TI-701 A } 5) \\
\text { SERVICE AIR HDR TEMP }\end{array}$ & & & & $90.120 \mathrm{~F}$ & The \\
\hline $\begin{array}{l}\text { DUTY CYCLE } \\
\text { (LOAD TIME/TOTAL T IME) }\end{array}$ & & & 27 & & $x$ \\
\hline RELATIVE HUMIDITY & & & $68 \%$ & & \\
\hline ATMOSPHERIC PRESSURE & & & 29.3611 & & \\
\hline INSIDE TENP, $(\mathrm{N} / 01 \mathrm{\gamma})$ & & & 68 & 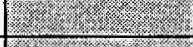 & $\pi$ \\
\hline $\begin{array}{l}\text { ouTSIDE TEMP } \\
\text { (OUTSIDE } 701-A)\end{array}$ & V & & $43 F$ & & \\
\hline
\end{tabular}

* Call weather station for required information at 373-2875

Note: If readings are outside designated ranges, Notify Test Director. 
HNF-SD-WM-OTR-231, Rev 0

Page 83 of 165

\section{8-HOUR ON-LINE TEST DATA SHEET}

FOR SA-CMP-801

\begin{tabular}{|c|c|c|c|c|c|}
\hline PARAMETER & DATE & TINE & REAOING & EXPECTED RANGE & INIT \\
\hline $\begin{array}{l}\text { APDI } 801 \text { (DPt } 701 / 2=1) \\
\text { CMP } 801 \text { SUCT FL D/P }\end{array}$ & $3 / 10 / 97$ & 1320 & & K6 Ps I & $T 7$ \\
\hline $\begin{array}{l}\text { SA-PI-801 (PI-701 A-2) } \\
\text { REMOTE BRG OIL PRESS }\end{array}$ & & & & $15-4060 \%$ Ps & $\pi e$ \\
\hline $\begin{array}{l}\text { SA-PI-805 (PI-701-A-1) } \\
\text { INTER STG DISCH PRESS }\end{array}$ & & & 26 & $20-40$ pSI & TK \\
\hline $\begin{array}{l}\text { GLY-FG-802 (SFI-701-AL4) } \\
\text { SA-CHP-801 HP SIDE GLY FLO }\end{array}$ & & & & Flon r , & tk \\
\hline $\begin{array}{l}\text { SA-PI-803 (PI-701-A-4) } \\
\text { CHP 801 DISCH PRESS }\end{array}$ & & & & $85-105 \mathrm{pst}$ & Tk \\
\hline $\begin{array}{l}\text { SA-TI-801 (TI-701-A-2) } \\
\text { CMP } 801 \text { SA DISCH TEMP }\end{array}$ & & & & $90-120 \mathrm{~F}$ & $T K$ \\
\hline $\begin{array}{l}\text { SA-SG-801 } \\
\text { SA CMP OIL LVL }\end{array}$ & & & & ABOVE IARK & TK \\
\hline $\begin{array}{l}\text { GLY-TI-803 (TI-701-A-7) } \\
\text { SA-CMP-801 GLY INLET TEMP }\end{array}$ & & & & $90-120 \%$ & Th \\
\hline $\begin{array}{l}\text { GLY-TI-801 (II-701 } /-9) \\
\text { SA-CHP-801 GLY DISCH TEMP. }\end{array}$ & & & 81 & $90-120 \mathrm{~F}$ & \\
\hline 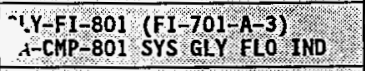 & & & $\theta 0$ & K67 GP & $7 x$ \\
\hline $\begin{array}{l}\text { SA-PISL-801 ( }(\mathrm{PI}-701-\mathrm{A}-1) \\
\text { SA HDR LO PRESS A A RRH }\end{array}$ & & & & 90-100. PS I & tie \\
\hline $\begin{array}{l}\text { SA-TI-805 (TI-701-A } 5) \\
\text { SERVICE AIR HDR IEPP. }\end{array}$ & & & & $90-120 \%$ & The \\
\hline $\begin{array}{l}\text { DUTY CYCLE } \\
\text { (LOAD TIME/TOTAL TIME) }\end{array}$ & & & 411 & & the \\
\hline RELATIVE HUMIDIY & & & $68 \%$ & (2. & \\
\hline ATMOSPHERIC PRESSURE & & & $29.36^{\circ}$ & (2) & \\
\hline INSIDE TEMP (IN $701-A)$ & & & 70 & & the \\
\hline $\begin{array}{l}\text { OUTSIDE TEMP. } \\
\text { (OUTSIDE } 701-A \text { ) }\end{array}$ & 4 & & $43 F$ & & \\
\hline
\end{tabular}

* Call weather station for required information at 373-2875

Note: If readings are outside designated ranges, Notify Test Director. 
HNF-SD-WM-OTR-231, Rev 0

Page 84 of 165

\section{8-HOUR ON-LINE TEST DATA SHEET (Cont.)}

FOR AIR DRYER/COOLING SKID

\begin{tabular}{|c|c|c|c|c|c|}
\hline PARAMETER & DATE & TIME & READNG & EXPECTED RANGE: & INIT \\
\hline GLY PUMP RUNMING & $3 \mid r d a n$ & $13^{20}$ & 2 & 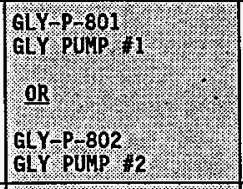 & TK \\
\hline $\begin{array}{l}\text { GLY-PI-BO1 (PI-701-A-17) } \\
\text { GLY PP SUCT PRES }\end{array}$ & & & 6 & o-10 Psi & $\mathbb{R}$ \\
\hline $\begin{array}{l}\text { GLY-PI- } 803 \text { (PI-701-A-16) } \\
\text { GLY PP DISCH PRESS }\end{array}$ & & & 57 & $65-75$ PSI: & Dre \\
\hline $\begin{array}{l}\text { GLYTI-805 (TI-701-A-13) } \\
\text { GLY CIRC PUMP SUCT TEMP }\end{array}$ & & & 91 & $100-120 \%$ & $T K$ \\
\hline $\begin{array}{l}\text { GLYTI-806 (TI-701-A 6) } \\
H X-801 \& H X-802 \text { OUTLET TEMP }\end{array}$ & & & 96 & $100-120 \mathrm{~F}$ & tK \\
\hline $\begin{array}{l}\text { GLY } L L G-801 \text { (LGL } 701-A-3) \\
\text { EXPANSTON TK } L V \text {. }\end{array}$ & & & $3 / 4$ & $1 / 3-2 / 3$ FuLL & $\pi k$ \\
\hline $\begin{array}{l}\text { IA-PI-819, } \\
\text { IA TUR } 801 \text { PRESS }\end{array}$ & & & 96 & $80-105 \mathrm{PSI}$ & $\pi$ \\
\hline $\begin{array}{l}\text { IA-PI-820 } \\
\text { IA PURGE PRESS }\end{array}$ & & & 45 & $40-60 \mathrm{psI}$ & $\pi x$ \\
\hline $\begin{array}{l}\text { IA-PI } 818 \\
\text { IA TRR } 802 \text { PRESS }\end{array}$ & & & $\theta$ & $80-105 \cdot \mathrm{PSI}$ & $\pi k$ \\
\hline $\begin{array}{l}\text { IA FI } 801 \text { ( }(\mathrm{Fl}-701-\mathrm{A}-2) \\
\text { IA FLON INDICATOR. }\end{array}$ & & & 20 & 45-90 CFI & $\pi<$ \\
\hline $\begin{array}{l}\text { IA-PI-821 (PI-701-A-12) } \\
\text { IA-PCV-802 DISCH PRESS }\end{array}$ & & & 46 & $30-45 \mathrm{psi}$ & $\pi$ \\
\hline $\begin{array}{l}\text { SA-PI-810 (PI-701-A-11) } \\
\text { SA REG DISCH PRESS }\end{array}$ & & & 41 & $25-45 \cdot \mathrm{PSI}$ & TK \\
\hline $\begin{array}{l}\text { BLOW DOWN PRE TLER, } \\
\text { AFTERFILTER, RECENER TAYKS }\end{array}$ & & & 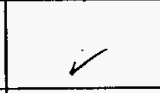 & (1.) & $\pi$ \\
\hline V. & t & $t$ & & 1 & \\
\hline
\end{tabular}


HNF-SD-WM-OTR-231, Rey 0

Page 85 of 165

\section{8-HOUR ON-LINE TEST DATA SHEET}

FOR SA-CHP-801

\begin{tabular}{|c|c|c|c|c|c|}
\hline 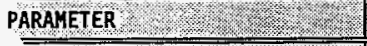 & DArE & TIME & READING & EXPECTED RANGE & INIT \\
\hline $\begin{array}{l}A-\mathrm{POI}-801 \text { (DPI-701-A-1) } \\
\text { CMP } 801 \text { SUCT FLT D } / \mathrm{P}\end{array}$ & $3 / 15 / 9$ & 1420 & & 6. pst & $\mathbb{R}$ \\
\hline $\begin{array}{l}\text { SA-PI-801 (PI-7O1-A- } 2 \text { ) } \\
\text { REMOTE BRG OIL PRESS }\end{array}$ & & & 2 & $15.69 .65 \mathrm{PSI}$ & T/C \\
\hline $\begin{array}{l}\text { SA-PI-805 (PI-701-A-1) } \\
\text { INTER STG DISCH PRESS }\end{array}$ & & & & $20-40 . P S T$ & $T_{k}$ \\
\hline $\begin{array}{l}\text { GLY-FG-802 (SFI } 701-A-4) \\
\text { SA-CMP-801 HP STOE G Y FLO }\end{array}$ & & & & ron r. & \\
\hline $\begin{array}{l}\text { SA-PI-803 (PI-701-A-4) } \\
\text { CHP } 801 \text { DISCH PRESS }\end{array}$ & & & & 85-105 PSI & $T K$ \\
\hline $\begin{array}{l}\text { SA-TI-801 (T I-701-A-2) } \\
\text { CMP } 801 \text { SA DISCH TEMP. }\end{array}$ & & & & $90-120 \%$ & $T x$ \\
\hline $\begin{array}{l}S A-S G-801 \\
S A \text { CHP OIL LVL }\end{array}$ & & & & ABOVE MARK & $-17 c$ \\
\hline $\begin{array}{l}\text { GLY-TI-803 (II-701-A-7) } \\
\text { SA-CHP-801 GLY INLET TEMP }\end{array}$ & & & 10 & $90-120 \%$ & $7 k$ \\
\hline $\begin{array}{l}\text { GLY-TI-801 (T1-701-A-g) } \\
\text { SA-CHP-801 GLY DISCH TEMP. }\end{array}$ & & & 100 & $90-120 \cdot \mathrm{F}$ & TK \\
\hline $\begin{array}{l}-\mathrm{Y} / \mathrm{FI}-801 \text { ( }\left(1 \mathrm{I}-701-\mathrm{A}_{-3}\right) \\
\mathrm{A} \text {-CMP-801 SYS GLY FLO IND }\end{array}$ & & & (1) & $<67 \mathrm{GPH}$ & Rx \\
\hline $\begin{array}{l}\text { SA-PISL-801 (PI-70I A-1) } \\
\text { SA HDR LO PRESS ALARH }\end{array}$ & & & & $90-100 \mathrm{PSI}$ & $T x$ \\
\hline $\begin{array}{l}\text { SA-TI-805 (TI-70I-A-5) } \\
\text { SERYICE AIR HDR TEMP }\end{array}$ & & & & $90-120 \%$ & $7 K$ \\
\hline $\begin{array}{l}\text { DUTY CYCLE, } \\
\text { (LOAD TIME/TOTAL TINE) }\end{array}$ & & & & & $T R$ \\
\hline \multicolumn{6}{|l|}{ RELATIVE HUMTOITY } \\
\hline \multicolumn{6}{|l|}{ ATMOSPHERIC PRESSURE } \\
\hline INSIDE TEMP (IN 701- A) & & & 76 & 18. & $T X$ \\
\hline $\begin{array}{l}\text { OUTSIDE TEMP. } \\
\text { (OUTSIDE } 701-A)\end{array}$ & & & & & \\
\hline
\end{tabular}

* Call weather station for required information at $373-2875$

Note: If readings are outside designated ranges, Notify Test Director. 
HNF-SD-WM-OTR-231, Rey 0

Page 86 of 165

\section{8-HOUR ON-LINE TEST DATA SHEET (cont.)}

R AIR DRYER/COOLING SKID

\begin{tabular}{|c|c|c|c|c|c|}
\hline PARALETER : & DATE & TIME & READING & EXPECTED RANGE & INII \\
\hline GL & $3 / 10 / 4 d$ & 1420 & & 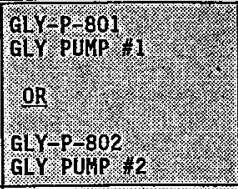 & TK \\
\hline 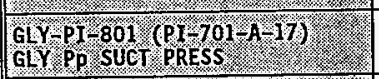 & & & 9 & $\mathrm{O}=1 \mathrm{p} \mathrm{psi}$ & The \\
\hline $\begin{array}{l}\text { GYY } P 1-803 \text { (PI } 101-A-16) \\
\text { GY PP DISCH PRESS }\end{array}$ & & & 60 & $65-75 . \mathrm{psi}$ & $t x$ \\
\hline $\begin{array}{l}\text { GLY TI } 805 \text { (T TO1-A } 13) \\
\text { GY Y CIRC PUAP SUCT TEMP) }\end{array}$ & & & & $100-120 \mathrm{~F}$ & The \\
\hline 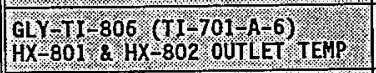 & & & $\ln 4$ & $100-120 \mathrm{~F}$ & $T x$ \\
\hline 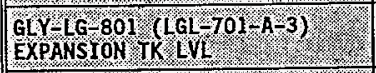 & & & $7 / 8$ & $1 / 3-2 / 3$ Fut & $T k$ \\
\hline $\begin{array}{l}\text { TA PI } 819 \\
\text { A TTR BOI PRESS }\end{array}$ & & & 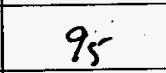 & $80-105$ ? & TK \\
\hline $\begin{array}{l}\text { IA PI I G20 } \\
\text { IA PURGE PRESS }\end{array}$ & & & & $40=60 \cdot \mathrm{pST}$ & TK \\
\hline $\begin{array}{l}\text { IAPI } 818 \text { IA } \\
\text { IA IRR } 802 \text { PRESS }\end{array}$ & & & & $80-105 \cdot \mathrm{PSI}$ & $+x$ \\
\hline 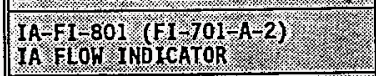 & & & $20 \frac{20}{x}$ & $45-90 \mathrm{cFI}$ & th \\
\hline 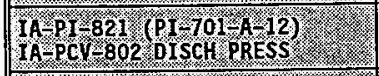 & & & 42 & $30-45$ psI & $t x$ \\
\hline 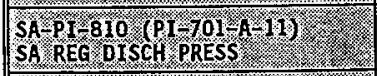 & & & 40 & $25-45 \cdot ?$ & Tk \\
\hline $\begin{array}{l}\text { BLOH DOWW PREFILTER, } \\
\text { AFTERFILTER, REGEIVR TASKS }\end{array}$ & & & & & $4 x$ \\
\hline 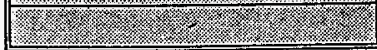 & & $L$ & & 12 & \\
\hline
\end{tabular}




\section{8-HOUR ON-LINE TEST DATA SHEET}

\begin{tabular}{|c|c|c|c|c|c|}
\hline PARAMETER & DATE. & TIHE. & READING & EXPECTED RANGE & $1 \mathrm{NIT}$ \\
\hline 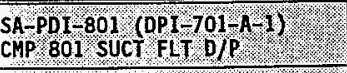 & $3 \operatorname{lioln}$ & 1520 & & K6. pst & D) \\
\hline $\begin{array}{l}\text { SA-PI } 801 \text { (PI-701-A-2) } \\
\text { REMOTE BRG OIL PRESS }\end{array}$ & & & & $15-4965$ PST & \\
\hline $\begin{array}{l}\text { SA-PI }-805 \text { (PI-70I-A-1) } \\
\text { INTER STG DISCH PRESS. }\end{array}$ & & & 2 & $20-40$ PSI & \\
\hline $\begin{array}{l}\text { GLY-FG-802 (SFI-701-A-4) } \\
\text { SA-CHP-801 HP SIDE GLY FLO }\end{array}$ & & & & Flow $\%$ & \\
\hline 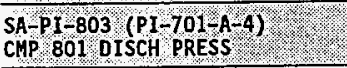 & & & & $85-105$ PST & \\
\hline $\begin{array}{l}\text { SA-TI } 801 \text { (TI 701-A-2) } \\
\text { CMP } 801 \text { SA DISCH TEMP }\end{array}$ & & & & $90-120 \mathrm{~F}$ & \\
\hline $\begin{array}{l}\text { SA-SG-801 } \\
\text { SA CHP OIL LVL }\end{array}$ & & & & ABOVE MARK & \\
\hline $\begin{array}{l}\text { GLY TI-803 (TI-701-A-7) } \\
\mathrm{CA}-\mathrm{CHP}-801 \text { GLY INLET IENP. }\end{array}$ & & & & $90-120 \mathrm{~F}$ & \\
\hline $\begin{array}{l}\text { GLY TI } 801 \text { (TI-701-A-9) } \\
\text { SA-CMP-801 GLY DISCH IEMP }\end{array}$ & & & & $90-120 \%$ & \\
\hline $\begin{array}{l}\text { GLY-FI-801 (FI-701-A-3) } \\
\text { SA-CMP-801 SYS GY FLO IND }\end{array}$ & & & $r$ & $\mathrm{~K} 67 \% \mathrm{GPH}$ & \\
\hline $\begin{array}{l}\text { SA-PISL-801 (PI- 701 A } 1) \\
\text { SA HDR LO PRESS ALARH }\end{array}$ & & & & $90-100 . \mathrm{PSI}$ & \\
\hline $\begin{array}{l}\text { SA-TI-805 (TI-701-A-5) } \\
\text { SERVICE AIR HDR TEMP. }\end{array}$ & & & & $90-120 \% \mathrm{~F}$ & \\
\hline $\begin{array}{l}\text { DUTY CYCLE, } \\
\text { (LOAD TIME/TOTAL TIME) }\end{array}$ & & & & & \\
\hline RELATIVE HUMIDIY & $n A$ & & & 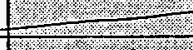 & \\
\hline ATMOSPHERIC PRESSURE & & & & & \\
\hline INSIDE TEMP (IN 701-A) & & & 76 & 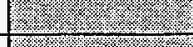 & 2 \\
\hline $\begin{array}{l}\text { OUTSIDE TEMP } \\
\text { (OUTSIDE TOI-A) }\end{array}$ & & & & 1.2 .8 & \\
\hline
\end{tabular}

* Call weather station for required information at 373-2875

Note: If readings are outside designated ranges, Notify Test Director. 


\section{8-HOUR ON-LINE TEST DATA SHEET (cont.)}

3 AIR DRYER/COOLING SKID

\begin{tabular}{|c|c|c|c|c|c|}
\hline PARAMETER & DATE & TtMe & READING & EXPECTED RANGE & INII \\
\hline 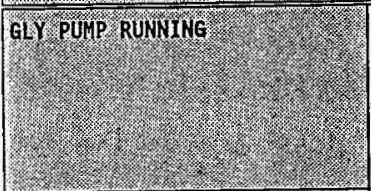 & $3 / 10 / 47$ & 1520 & & $\begin{array}{l}\text { GGY } 801 \\
\text { GL PUMP } \\
0 \mathrm{OR} \\
\text { GY P } 802 \\
\text { GY PUMP 2 }\end{array}$ & \\
\hline 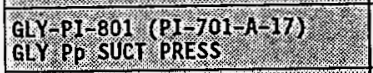 & & . & 8 & $0.10 .9 s 1$ & \\
\hline 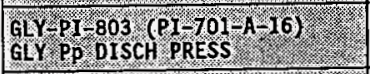 & & & $60^{\circ}$ & $65-75 \mathrm{PST}$ & \\
\hline 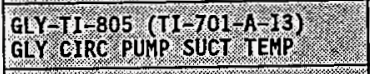 & & & & $100 \div 120$ & \\
\hline 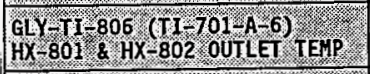 & & & & $100-120 \mathrm{~F}$ & \\
\hline 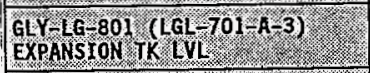 & & & & $1 / 3-2 / 3$ FUI & \\
\hline $\begin{array}{l}\text { ARI } 819 \\
\text { TWR } 801 \text { PRESS }\end{array}$ & & & 90 & $80 \div 105$ PSI & \\
\hline $\begin{array}{l}\text { GAVI-820? } \\
\text { IA PURGE RRES }\end{array}$ & & & & $40=60.957$ & \\
\hline $\begin{array}{l}\text { IA PI } 818 \text {. } 18 \text { PRES } \\
\text { IA TWR } 802 \text { PR }\end{array}$ & & & & $80-105.95]$ & \\
\hline 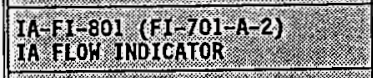 & & & & 45. $90 . \mathrm{CFH}$ & \\
\hline 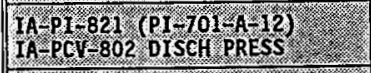 & & & & $30-45 \mathrm{psI}$ & \\
\hline 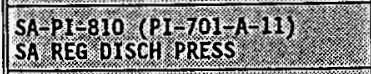 & & & 0 & $25-45.85$ & \\
\hline 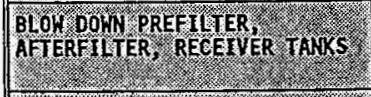 & & & & & \\
\hline lर & & & & & \\
\hline
\end{tabular}


HNF-SD-WM-OTR-231, Rey 0

Page 90 of 165

48-HOUR ON-LINE TEST DATA SHEET (Cont.)

IR AIR DRYER/COOLING SKID

\begin{tabular}{|c|c|c|c|c|c|}
\hline PARALITER & DATE - & THE & READING & EXPECTIED RALG & INIII \\
\hline GLP PUMP RUNAING ( & $1 / 10 / 9$ & 1630 & & 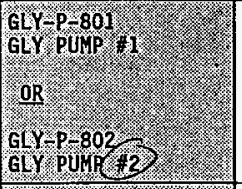 & \\
\hline 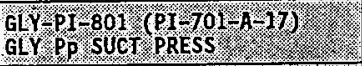 & & & & $010 . P S 1$ & \\
\hline 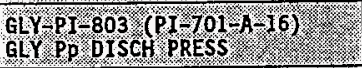 & & & & $65-75.95$ & \\
\hline $\begin{array}{l}\text { GLY TI } 805 \text { (TI } 701-A-13) \\
\text { GYY CIRC PUMP SUCT TENP }\end{array}$ & & & & $100-120 \%$ & \\
\hline 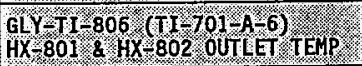 & & $\cdot$ & & $100-120$ & \\
\hline 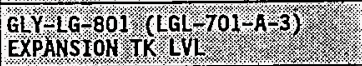 & & & & $1 / 3-2 / 3$, rutu & \\
\hline $\begin{array}{l}\text { IA PI- } 819 . \\
\mathrm{AA} / \mathrm{TR} 801 \text { PRESS }\end{array}$ & & & & $80 \div 105 \mathrm{ps}$ & \\
\hline $\begin{array}{l}\text { IA PPI } \\
\text { IA PURG }\end{array}$ & & & & $40=60 \mathrm{psI}$ & \\
\hline $\begin{array}{l}\text { IA PI } 818 \text {. } \\
\text { IA THA } 802 \text { PRESS }\end{array}$ & & & & $80.105 . P S T$ & \\
\hline $\begin{array}{l}\text { IA }=1,801 \text { (F) } 701-1-2) \\
\text { IA FLOH IHDICATOA }\end{array}$ & & & & 45-90. CFH & \\
\hline 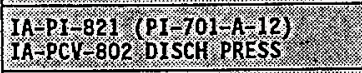 & & & & $30-45$ PST & \\
\hline $\begin{array}{l}\text { SA PI } 810 \text { (PIRTI) } \\
\text { SA REG OISCH PRESS }\end{array}$ & & & & $25.45 \times 18$ & \\
\hline 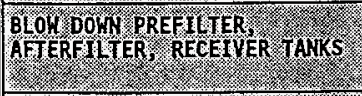 & & . & & & \\
\hline 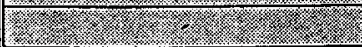 & & & & (1) & \\
\hline
\end{tabular}




\section{8-HOUR ON-LINE TEST DATA SHEET}

FOR SA-CMP-801

\begin{tabular}{|c|c|c|c|c|c|}
\hline 'ARAMETER & DATE & MIME & READING & EXPECTED RANGE & INIT \\
\hline $\begin{array}{l}\text { iA-PDI-801 (DPI-701-A } 1) \\
\text { KMP } 801 \text { SUCT FLT D/P }\end{array}$ & $3-10-97$ & 1720 & & Ko. PST. & \\
\hline $\begin{array}{l}\text { SA-PI BO2 (PI-70I-A-2) } \\
\text { ZEMOTE BRG OII PRESS }\end{array}$ & -1 & & & $15.465 \mathrm{pst}$ & \\
\hline $\begin{array}{l}\text { SA-PI } 805 \text { (PI-701-A-1) } \\
\text { INTER STG DISCH PRESS }\end{array}$ & & & 2 & $20-40 \mathrm{PSI}$ & \\
\hline $\begin{array}{l}\text { ILY-FG-802 (SFI-701 A-4) } \\
3 A-C H P-801 \text { HP SIDE GLY FLO }\end{array}$ & & & $\checkmark$ & ELOW & \\
\hline $\begin{array}{l}\text { SA-PI-803 (PI-701-A-4) } \\
\text { MPP } 801 \text { DISCH PRESS }\end{array}$ & & & 9 & $85-105$ PSI & \\
\hline $\begin{array}{l}3 \mathrm{~A}-\mathrm{TI} 801 \text { (TI } 701-\mathrm{A}-2) \\
\mathrm{MP} \text { P } 801 \text { SA DISCH TEMP }\end{array}$ & & & & $90-120 \mathrm{~F}$ & \\
\hline $\begin{array}{l}\mathrm{SA-SG-801} \\
\mathrm{SA} \text { CMP } 0 \mathrm{IL} \text { LVL }\end{array}$ & & & & ABOYE WARK & \\
\hline $\begin{array}{l}3 L Y-T I-803 \text { (TI-701-A-7) } \\
\text { S4-CHP-801 GLY INLET TEMP }\end{array}$ & & & & $90-120 \%$ & \\
\hline $\begin{array}{l}\mathrm{SL} Y-T 1-801 \text { (TI-701-A-g) } \\
\text { SA-CMP-801 GLY DISCH TEMP. }\end{array}$ & & & & $90-120 \%$ & \\
\hline $\begin{array}{l}\text { GLY-FI-801 (FI-701-A-3) } \\
S A-C H P-801 \text { SYS GLY FLO IND }\end{array}$ & & & & $867.6 P H$ & \\
\hline $\begin{array}{l}\text { SA-PISL }-801 \text { (PI-701-A } 1) \\
\text { SA HDR LO PRESS ALARM }\end{array}$ & & & & 90. 100 Ps I & \\
\hline $\begin{array}{l}\text { SA-TI-805 (TI-701-A-5) } \\
\text { SERVICE AIR HDR TEMP. }\end{array}$ & $3-10-97$ & & & $90-120 \%$ & $x_{\sigma}$ \\
\hline $\begin{array}{l}\text { DUTY CYCLE } \\
\text { (LOAD TIME TOTAL TIME) }\end{array}$ & & & & & \\
\hline RELATIVE HUMIUITY & & & & 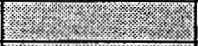 & \\
\hline ATHOSPHERIC PRESSURE & & & & 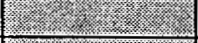 & \\
\hline INSIDE TEMP / (IN 701-A) & & & & 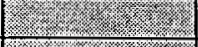 & \\
\hline $\begin{array}{l}\text { OUTSIDE TEMP. } \\
\text { (OUTSIDE } 701-\mathrm{A} \text { ). }\end{array}$ & & & & 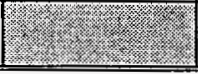 & \\
\hline
\end{tabular}

* Call weather station for required information at 373-2875

Note: If readings are outside designated ranges, Notify Test Director.

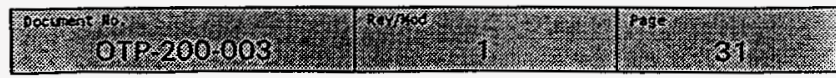


HNF-SD-WM-OTR-231, Rev 0

Page 92 of 165

\section{8-HOUR ON-LINE TEST DATA SHEET (cont.)}

JR AIR DRYER/COOLING SKID

\begin{tabular}{|c|c|c|c|c|c|}
\hline PARAHET ER & DATE & GME & READING & EXPECTED RANGE & 1NIT \\
\hline 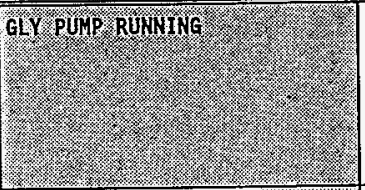 & $3 / 10 k_{1}$ & & & 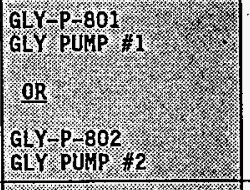 & \\
\hline 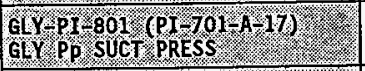 & & & 8 & $0.10 \mathrm{PSI}$ & \\
\hline $\begin{array}{l}\text { GY PI } 803 \text { (PI } 701 \text { R } 16) \text { ) } \\
\text { GL PP DISCH PRESS }\end{array}$ & & & 6 & 65-75 PSI & \\
\hline $\begin{array}{l}\text { G(Y-TI-805 (TI } 701-13) \\
\text { GLY CIRC PUMP SUGT TEMP }\end{array}$ & & & $a$ & 100-120.F & \\
\hline $\begin{array}{l}G L Y T L \\
H X-801\end{array}$ & & & & $100-120 \%$ & \\
\hline 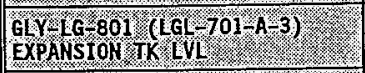 & & & & $1 / 3-2 / 3 . \% 11$ & \\
\hline $\begin{array}{l}\text { IA P I } 819 \\
\text { A YWR } 801 \text { PRESS }\end{array}$ & & & & $80-105 . \mathrm{PSI}$. & \\
\hline $\begin{array}{l}\text { IAPT } 820 \text { PRES } \\
\text { IA PURGE PRE }\end{array}$ & & & & 40-60. PS I & \\
\hline $\begin{array}{l}\text { IA PI } 818,0 \\
\text { IA IMR } 802 \text { PRESS }\end{array}$ & & & & $80.105 \cdot P S 1$ & \\
\hline 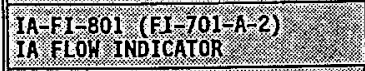 & & & 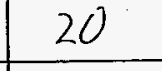 & $45-90, \mathrm{CFH}$ & \\
\hline 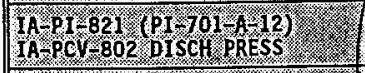 & & & & $30-45 \mathrm{PSI}$ & \\
\hline 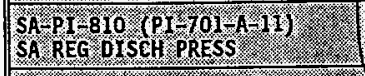 & & & & 25-45: PSI & \\
\hline $\begin{array}{l}\text { BLOW DOWN PRETLMER, } \\
\text { AFTERFTMTER. RECEIVER TANKS }\end{array}$ & 3hiolar & & & & \\
\hline 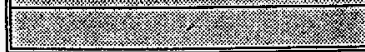 & & & & (2) & \\
\hline
\end{tabular}


HNF-SD-WM-OTR-231, Rey 0

Page 93 of 165

INFORMATION COPY

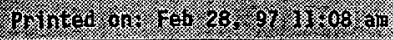

\section{8-HOUR ON-LINE TEST DATA SHEET}

\begin{tabular}{|c|c|c|c|c|c|}
\hline PARAMEIER & DATE & 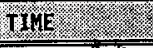 & READING & EXPECTEQ RANGE & WIT. \\
\hline 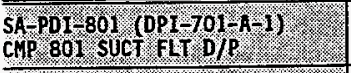 & $3-10 \cdot 9$ & 0630 & 1 & 6. PSI & \\
\hline 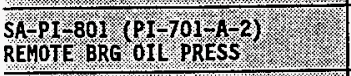 & 1 & 1 & & 15766\% pSI , & $1 / \theta$ \\
\hline $\begin{array}{l}\text { SA-PI } 805 \text { (PI-701/Al) } \\
\text { INTER STG DISCH PRESS }\end{array}$ & & & & $20-40$ pS & \\
\hline 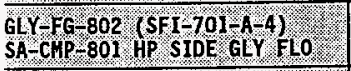 & & & & PLOW & \\
\hline $\begin{array}{l}\text { SA-PI } 803 \text { (PI-70I-A } 4) \\
\text { CHP } 801 \text { DISCH PRESS. }\end{array}$ & & & & 85-105 & \\
\hline $\begin{array}{l}\text { SA-TI } 801 \text { (TI-701 A-2) } \\
\text { CHP } 801 \text { SA DISCH TEMP }\end{array}$ & & & & 90.120. & \\
\hline $\begin{array}{l}\text { SA-SG } 801 \\
\text { SA CFP OIL LVL }\end{array}$ & & & $O K$ & ABOVE. & \\
\hline 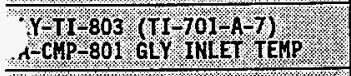 & & & 0 & $90-120$. & \\
\hline 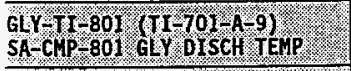 & & & 96 & 90.120 .9 & \\
\hline 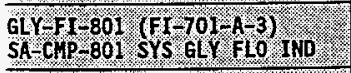 & & & & K67.GPM & \\
\hline 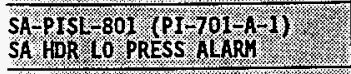 & & & & 90, $100 . \mathrm{PST}$ & \\
\hline 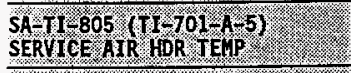 & & & & $90-120.7$ & \\
\hline $\begin{array}{l}\text { DUTY CYCLE } \\
\text { (LOAD THETAL, TIME) }\end{array}$ & & & & & \\
\hline RELATHE KGHIDIT & & & 23 & & \\
\hline ATHOSPRERIC PRESSURE & & & 22 & t. & \\
\hline IHSIOE IEL $(\mathrm{IN} 701) \mathrm{N}$ ) & & & $7^{6}$ & . & 8 \\
\hline $\begin{array}{l}\text { OUTSIDE TEMP } \\
\text { (OUTSIDE } 701-A)\end{array}$ & 1 & $=$ & & & 18 \\
\hline
\end{tabular}

* Call weather station for required information at 373-2875

Note: If readings are outside designated ranges, Notify Test Director.

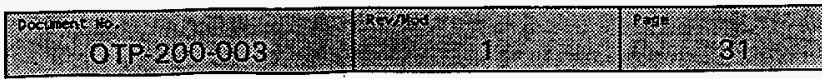




\section{8-HOUR ON-LINE TEST DATA SHEET (Cont.)}

OR AIR DRYER/COOLING SKID

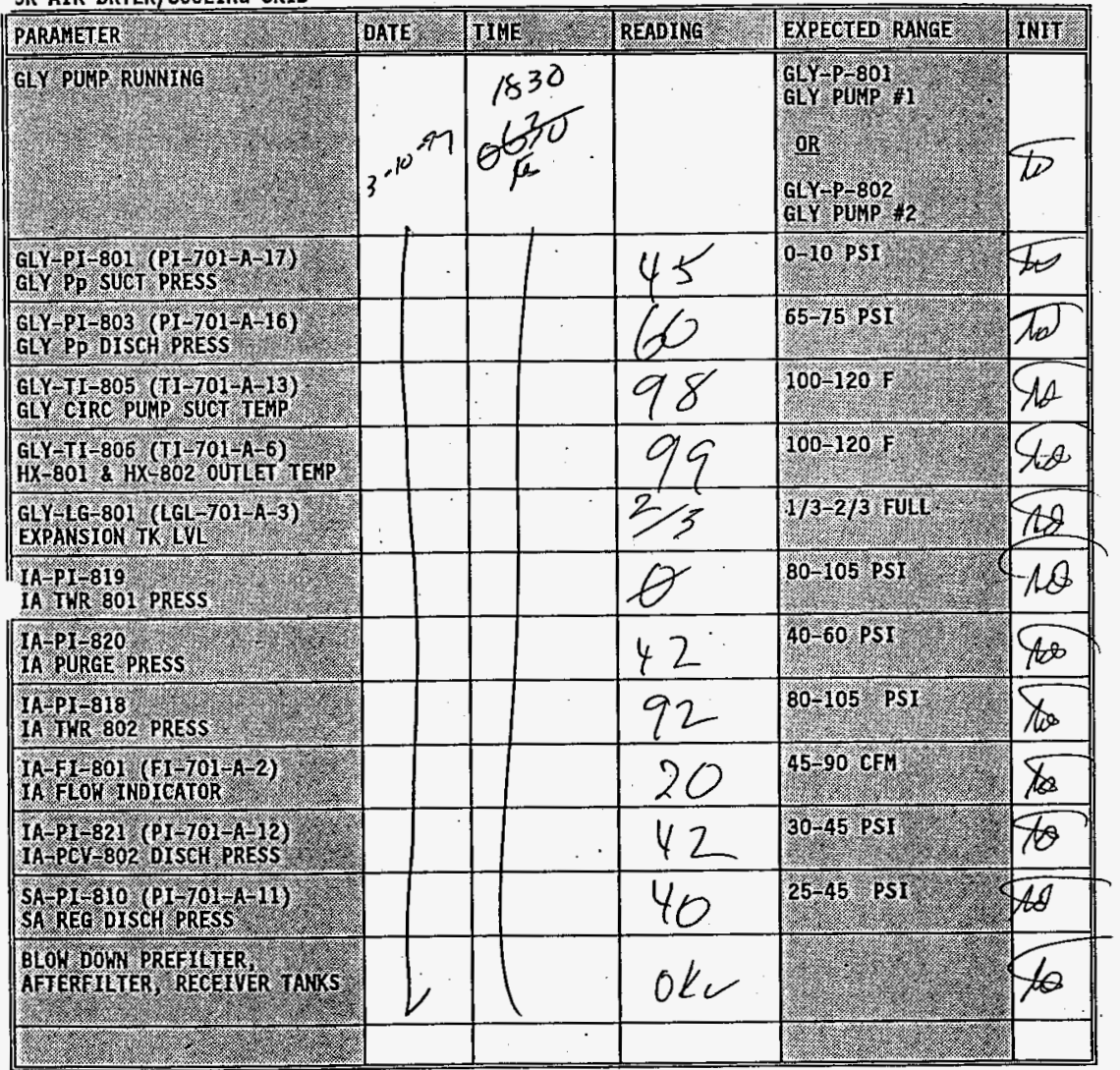


HNF-SD-WM-OTR-231, Rev 0

Page 95 of 165

\section{INEORMATION GOPY}

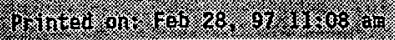

\section{8-HOUR ON-LINE TEST DATA SHEET}

FOR SA-CHP-801

\begin{tabular}{|c|c|c|c|c|c|}
\hline ARAMEIER & $\mathrm{DATE}$ & TIME & $\mathrm{BEAD}$ & EXPECTED RANGE & MI \\
\hline 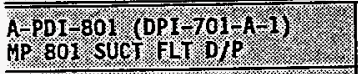 & $3-10-97$ & 0730 & & K6, pSI & $2 \theta$ \\
\hline $\begin{array}{l}\text { API } 801 \text { (PI-701- } 2) \\
\text { EMOTE BRG OIL PRESS }\end{array}$ & & . & & 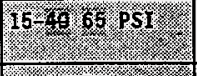 & $\pi$ \\
\hline $\begin{array}{l}\text { API } 805 \text { (PI } 701 \text { A A } 1) \\
\text { NTER STG DISCH PRESS }\end{array}$ & & & & $20=40.951$ & $\sqrt{10}$ \\
\hline 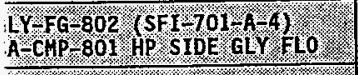 & & & & FLOW. & $\theta$ \\
\hline $\begin{array}{l}\text { A.PI-803 (PI-701-A } 4) \\
\text { BP } 801 \text { DISCH PRESS }\end{array}$ & & & & 85.105 & $\sqrt{10}$ \\
\hline 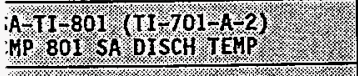 & & & & $90 \div 120$ & \\
\hline $\begin{array}{l}\text { A SG } 801 \\
\text { A CFP } 0 \mathrm{IL} \text { LVL }\end{array}$ & & & & ABOYE WAP & 40 \\
\hline 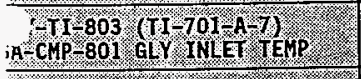 & & & & $90 \times 120 \%$ & \\
\hline 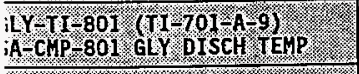 & & & & $90 \div 120.1$ & $\sqrt{10}$ \\
\hline 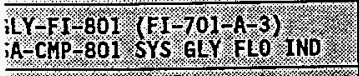 & & & $V_{h}$ & $667.6 \mathrm{p}$ & \\
\hline 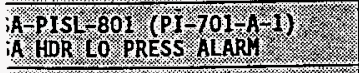 & & & 2 & $90.100 \%$ pst & 4 \\
\hline 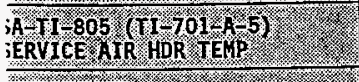 & $!$ & & 1 & $90=120.9$ & \\
\hline $\begin{array}{l}\text { WTY GYGE } \\
\text { WOAD WIME/TOTA TIME) }\end{array}$ & & & & & $\sqrt{8}$ \\
\hline KATIV HUMOU) & & & $x^{A}$ & & \\
\hline ITOSPLERTC PRESSURE - - - & & & $M A$ & 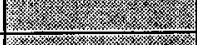 & \\
\hline NSIDE V N & & & 76 & & W \\
\hline $\begin{array}{l}\text { UTSTDE TEMP } \\
\text { OUTSIDE }(01-A)\end{array}$ & & & & & 4 \\
\hline
\end{tabular}

Call weather station for required information at 373-2875

Note: If readings are outside designated ranges, Notify Test Director.

(1) out of spec

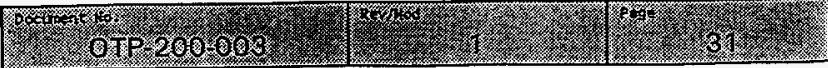


HNF-SD-WM-OTR-231, Rev 0

Page 96 of 165

48-HOUR ON-LINE TEST DATA SHEET (Cont.)

IR AIR DRYER/COOLING SKID

\begin{tabular}{|c|c|c|c|c|c|}
\hline PARAMRIER & DAIE & TLE : & READING - & EXPECTED RANGE & Wirt \\
\hline GY PUMP RUNAING & $3^{10^{.97}}$ & $\left\{\begin{array}{l}930 \\
0750\end{array}\right.$ & $\mathbb{1}^{2}$ & 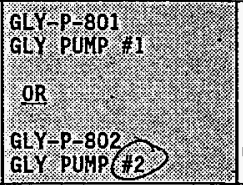 & $5 x$ \\
\hline $\begin{array}{l}\text { GLY PI } 801 \text { (PI } 701-A-17) \\
\text { GLY PP SUCT PRESS }\end{array}$ & & & 7. & O-10 PSI & $\pi$ \\
\hline $\begin{array}{l}\text { GLY PI-803 (PI 701-A-16)) } \\
\text { GY PP DISCH PRESS }\end{array}$ & & & 60 & 65-75. PSI & $T$ \\
\hline $\begin{array}{l}\text { GLY TI } 805 \text { (TI } 701-1 / 13) \\
\text { GLY CIRC PUP SUGT TEMP }\end{array}$ & & & & $100-120 \mathrm{~F}$ & Fet \\
\hline 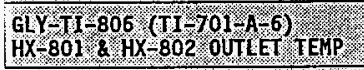 & & & & $100-120$ & 12 \\
\hline 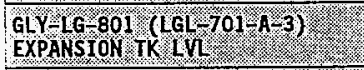 & & & 2 & $1 / 3-2 / 3$ & 70 \\
\hline $\begin{array}{l}\text { TA PI } 819 \text { I PRESS } \\
\text { IA THR } 801 \text {. }\end{array}$ & & & 92 & $80-105 \%$ & te \\
\hline $\begin{array}{l}\text { IAPI } 1220 \\
\text { IA PURGE PRESS }\end{array}$ & & & 42 & $40=60$ & $\pi 0$ \\
\hline $\begin{array}{l}\text { IA PI } 818 \\
\text { IA TWR } 802 \text { PRESS }\end{array}$ & & & & $80-105$ PS & $2 \pi$ \\
\hline $\begin{array}{l}\text { IA } 11801 \text { ( } 1 \text { IOI } \\
\text { IA FLO INDICATOR }\end{array}$ & & & 20 & $45-\mathrm{go} \mathrm{CFM}$ & 42 \\
\hline 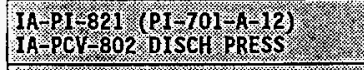 & & & 42 & $30=45$ PSI & $\dot{10}$ \\
\hline $\begin{array}{l}\text { SA-PI } 610 \text { (PI TO1-A-11) } \\
\text { SA REG DISCH PRESS }\end{array}$ & & & 40 & $25 \div 45: P S T$ & $\bar{\mu}$ \\
\hline 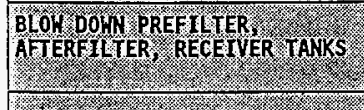 & $\angle$ & & & & $\mu^{0}$ \\
\hline 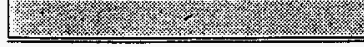 & & & & 12 & \\
\hline
\end{tabular}


HNF-SD-WM-OTR-231, Rev 0

Page 97 of 165

WORKING COPY

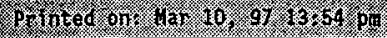

\section{8-HOUR ON-LINE TEST DATA SHEET}

:0R SA-CMP-801

\begin{tabular}{|c|c|c|c|c|c|}
\hline RAMETER & DATE & MHE. & READING & EXPECTED RANGE & INIT \\
\hline 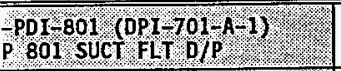 & $\begin{array}{c}3-10-97 \\
1\end{array}$ & $11: 30 / 2330$ & & $6, P S \mid$ & $\$ 0$ \\
\hline $\begin{array}{l}\text { PI-801 }(\mathrm{Pl}-701-\mathrm{A}-2) \\
\text { MOTE BRG OIL PRESS }\end{array}$ & & & & $15-46.65$ PSI & 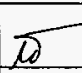 \\
\hline $\begin{array}{l}\text { PI-805 (PI-701-A-1) } \\
\text { TER STG DISCH PRESS }\end{array}$ & & & & $20-40 . \mathrm{PSI}$ & T2 \\
\hline $\begin{array}{l}\mathrm{Y}-\mathrm{FG}-802 \text { (SFI-701-A-4) } \\
-\mathrm{CHP}-801 \mathrm{HP} \text { SIDE GLY FLO }\end{array}$ & & & & FLON/ & 16 \\
\hline $\begin{array}{l}-\mathrm{PI} 803 \text { ( } \mathrm{PI}-701 \text { A } 4 \text { ) } \\
\mathrm{P} 801 \text { OISCH PRESS }\end{array}$ & & & & $85-105$ psI & The \\
\hline $\begin{array}{l}\text { T1-801 (TI-701-A-2) } \\
\text { P } 801 \text { SA DISCH TEMP }\end{array}$ & & & & $90-120 \mathrm{~F}$ & $\sqrt{18}$ \\
\hline $\begin{array}{l}-\mathrm{SG}-801 \\
\mathrm{CFP} 01 \mathrm{LVL}\end{array}$ & & & ok & ABOVE MARK & 20 \\
\hline $\begin{array}{l}Y-\mathrm{TI}-803 \text { (TI-701-A-7) } \\
-\mathrm{CHP}-801 \text { GLY INLET TEHP }\end{array}$ & & & & $90-120 \mathrm{~F}$ & 48 \\
\hline $\begin{array}{l}Y-\mathrm{TI}-801 \text { (TI- } 701-\mathrm{A}-9) \\
\mathrm{I-CHP}-801 \text { GLY DISCH TEMP. }\end{array}$ & & & & $90-120 \mathrm{~F}$ & 40 \\
\hline $\begin{array}{l}\text { Y-FI-801 (FI-701-A-3) } \\
\text { H-CMP-801 SYS GLY FLO IND. }\end{array}$ & . & & & K67 GPM & til \\
\hline $\begin{array}{l}\text {-PISL }-801 \text { (PI-701-A-1) } \\
\text { HDR LO PRESS ALARM }\end{array}$ & & & & $90-100 \mathrm{PSI}$ & LO \\
\hline $\begin{array}{l}\text {-TI-805 (TI-701-A-5) } \\
\text { RVICE AIR HDR TEMP }\end{array}$ & & & & $90-120 . F$ & 80 \\
\hline $\begin{array}{l}\text { TYYCYCLE } \\
\text { OAD TIME/TOTAL TIME) }\end{array}$ & & & & & 8 \\
\hline GATIVE HUMIDITY & & & $N A$ & & $\mathscr{E}$ \\
\hline MOSPHERIC PRESSURE & & & $\sqrt{2}$ & (1.). & 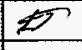 \\
\hline ISIDE TEMP (IN TO1 A) & & & 76 & & \\
\hline $\begin{array}{l}\text { ITSIDE TEMP. } \\
\text { JUTSIDE } 701-A \text { ) }\end{array}$ & $V$ & $V$ & 9 & & \\
\hline
\end{tabular}

* Call weather station for required information at $373-2875$

Note: If readings are outside designated ranges, Notify Test Director.

(1) Roads Botn $F^{\circ}$ AnC

out of SPQL 
HNF-SD-WM-OTR-231, Rev 0

Page 98 of 165

48-HOUR ON-LINE TEST DATA SHEET (Cont.)

R AIR DRYER/COOLING SKID

\begin{tabular}{|c|c|c|c|c|c|}
\hline PARANETER & DATE & TIML & READING & EXPECTED RANGE & WIT \\
\hline $\mathrm{GLY}$ PUMP RUNAING & $3^{-10 \cdot 97}$ & $\begin{array}{l}233^{\circ} \\
11 \cdot 30 \\
7^{\circ} \cdot \mathrm{m}\end{array}$ & & 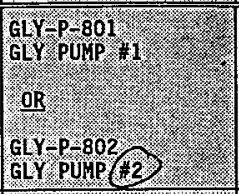 & $\sqrt{10}$ \\
\hline $\begin{array}{l}\text { GLYPI } 801 \text { (PI } 701=\mathrm{A}-17) \\
G L Y \text { P SUCT PRESS }\end{array}$ & & & 9 & $0-10.251$ & $\pi \theta$ \\
\hline $\begin{array}{l}\left.\text { GVYPI } 803 \text { (PI } 701-{ }^{-16}\right) \\
\mathrm{GLY} \text { PD DISCH PRESS }\end{array}$ & & & & $65-7.5 .951$ & 16 \\
\hline $\begin{array}{l}\text { GLY TI } 805(T 1-701-A=13) \\
\text { GLY CIRC PUMP SUCT. TEMP }\end{array}$ & & & & $100-120 \%$ & $\sqrt{12}$ \\
\hline 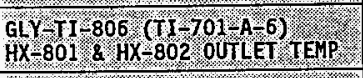 & & & & $100-120 . ?$ & 10 \\
\hline 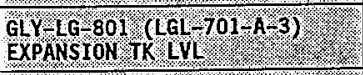 & & & $2 / 3^{\text {ok }}$ & $1 / 3-2 / 3, \mathrm{Fu}$ & $\sqrt{18}$ \\
\hline $\begin{array}{l}\text { TAPI } 819 \\
\text { A THR } 801 \text { PRESS }\end{array}$ & & & & $80-105.95$ & 12 \\
\hline $\begin{array}{l}\text { IA PI } 820 \\
\text { MA PURGE PRESS }\end{array}$ & & & & 40-60 PSI & 22 \\
\hline $\begin{array}{l}\text { IAPI } 818, \\
\text { IA THR } 802 \text { PRESS }\end{array}$ & & & 90 & $80-105 \times \mathrm{PSI}$ & 18 \\
\hline 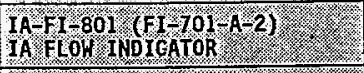 & & & 20 & 45.90. CEH & 62 \\
\hline $\begin{array}{l}\text { IAPI } 821 \text { (PI } 701 / 2,12) \\
1 \mathrm{~A}-\mathrm{PCl} 802 \text { DISCH PRESS }\end{array}$ & & & 42 & $30=45 \% \mathrm{psT}$ & 28 \\
\hline 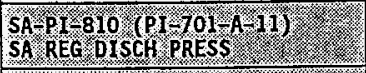 & & & 40 & $25-45$ PS & \\
\hline $\begin{array}{l}\text { BLOW DOWN RREFLTER, } \\
\text { AFIERFILTER, RECETER TANSS }\end{array}$ & & & & & 60 \\
\hline 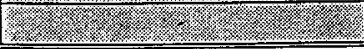 & 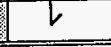 & $V$ & & 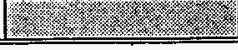 & \\
\hline
\end{tabular}


HNF-SD-WM-OTR-231, Rev 0

Page 99 of 165

\section{WORKING COPY

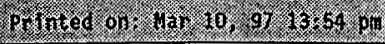

\section{8-HOUR ON-LINE TEST DATA SHEET}

FOR SA-CMP-801

\begin{tabular}{|c|c|c|c|c|c|}
\hline 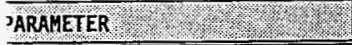 & DATE & T115 & READING & EXPECTED RANGE & INIT \\
\hline 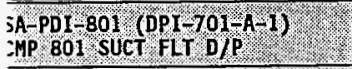 & $3 \cdot 11 \cdot 9$ & 330 A.p & & 66. PSI & ThC \\
\hline $\begin{array}{l}\text { SA-PI-801 (PI 701 A } 2) \\
\text { SEMOTE BRG OIL PRESS }\end{array}$ & & & $2 / \$ \pi x$ & $15-2605$ & $7 k$ \\
\hline $\begin{array}{l}\text { 3A-PI-805 (P1-701-A } 1) \\
\text { INTER STG DISCH PRESS }\end{array}$ & & & 26 & $20-40$ PSI & The \\
\hline $\begin{array}{l}\text { iLY-FG-802 (SFI }-701-A-4) \\
\text { SA-CMP-801 HP SIDE GLY FLO }\end{array}$ & & & & FLOW & The \\
\hline $\begin{array}{l}\text { SA-PI-803 (PI - 701-A-4) } \\
\text { KMP } 801 \text { DISCH PRESS }\end{array}$ & & & 91 & $85-105$ PSI & $\pi / c$ \\
\hline $\begin{array}{l}3 A-T 1-801 \text { ( }(1-701-A-2) \\
\text { ZMP } 801 \text { SA OISCH TEMP. }\end{array}$ & & & $\frac{2}{72} 20_{178}^{82 C}$ & $90 \div 120$ & nc \\
\hline $\begin{array}{l}3 \mathrm{~A}-\mathrm{SG}-801 \\
\mathrm{SA} \text { CMP } 01 \mathrm{~L} \text {, LVL }\end{array}$ & & & & ABOVE MARK & $\pi$ \\
\hline $\begin{array}{l}3 \mathrm{IL}-\mathrm{TI}-803 \text { ( } \mathrm{TI}-701-\mathrm{A}-7) \\
=\mathrm{A} \mathrm{CHP}-801 \text { GLY INLET TEAP. }\end{array}$ & & & & $90.120 \%$ & Thc \\
\hline $\begin{array}{l}\text { LV-TI-801 (TI-701-A-g) } \\
\text { SA-CMP-801 GLY DISCH TEMP }\end{array}$ & & & & $90-120 \%$ & $T C$ \\
\hline $\begin{array}{l}\text { ILY-FI - BOI (FI 701-A-3) } \\
\text { SA-CHP-BOI SYS GLY ELO IND }\end{array}$ & & & & $667 \mathrm{GPH}$, & Tic \\
\hline $\begin{array}{l}\text { SA-PISL } 801 \text { (PI }-701-A-1) \\
\text { SA HDR } \angle 0 \text { PRESS ALARM }\end{array}$ & & & & 901100 pSI & TK \\
\hline $\begin{array}{l}\text { SA-TI- } 805\left(\mathrm{TI}-701-{ }_{A}-5\right) \\
\text { SERVICE AIR HDR TEXP. }\end{array}$ & & & & $90-120 \mathrm{~F}$ & $T K$ \\
\hline $\begin{array}{l}\text { JUTY CYCEE } \\
\text { (LOAD TIME/TOTAL TIME) }\end{array}$ & & & 9 & 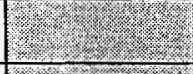 & tic \\
\hline BELATIVE HUMIDITY & & & $M / A$ & 1.2 .0 .20 & \\
\hline ATMOSPHERIC PRESSURE & & & $M / A$ & 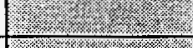 & \\
\hline INSIDE TENP. (IN $701-\mathrm{A})$ & & & 73 & 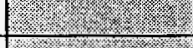 & $\pi c$ \\
\hline $\begin{array}{l}\text { JUTSIDE TEMP. } \\
\text { (OUTSIDE } 701-A \text { ). }\end{array}$ & & V & 36 & $y^{3}$ & T/c \\
\hline
\end{tabular}

* Call weather station for required information at $373-2875$

Note: If readings are outside designated ranges, Notify Test Director.

(1) out of spees. ok Per Loy book. 
HNF-SD-WM-OTR-231, Rev 0

Page 100 of 165

WORKING COPY

10.

48-HOUR ON-LINE TEST DATA SHEET

$5^{\infty} n^{80}$

FOR SA-CMP-801

\begin{tabular}{|c|c|c|c|c|c|}
\hline 'ARAMETER & DATE & TIKE & READING & EXPECTED & \\
\hline 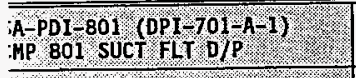 & -97 & 0730 & 2.0 & K6.PST & \\
\hline $\begin{array}{l}\text { :A-PI } 801 \text { (PI 701-A-2) } \\
\text { :EMOTE BRG OIL PRESS }\end{array}$ & $5 / 11 / 97$ & 0732 & 20 & $15-4065$ & \\
\hline $\begin{array}{l}\text { AA-PI-805 (PI-701-A-1) } \\
\text { NTER STG DISCH PRESS }\end{array}$ & $3 / 11 / 97$ & 0732 & 26 & $20-40$ PSI & \\
\hline $\begin{array}{l}\mathrm{LY}-F G-802 \text { (SFI-101-A-4) } \\
\mathrm{iA-CMP-801} \mathrm{HP} \mathrm{SIDE} \mathrm{GLY} \mathrm{FLO}\end{array}$ & $3 / 11 / 97=$ & 0732 & & FLOW & \\
\hline $\begin{array}{l}\text { AA-PI-803 (PI-701-A-4) } \\
\text { MP } 801 \text { DISCH PRESS }\end{array}$ & $3 / 11 / 97$ & 6733 & 97 & $85-105$ & \\
\hline $\begin{array}{l}\text { AA-T - } 801 \text { (II-701-A-2) } \\
\text { MP } 801 \text { SA DISCH TEMP }\end{array}$ & $3 / 11 / 97$ & 6733 & 180 & $90=120$ & \\
\hline $\begin{array}{l}3 A-S G-801 \\
\text { SA C CHP OIL LVL. }\end{array}$ & $3 / 4 /\{7$ & 0733 & & ABOVE Y & \\
\hline 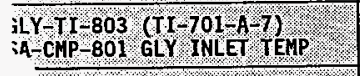 & $3 / 11 / 97$ & 0733 & & $90-120$ & \\
\hline $\begin{array}{l}\text { (-TI-BOI (TI-701-A-9) } \\
\text { A-CAP-801 GLY DISCH TEMP }\end{array}$ & $3 / 11 / 97$ & 0733 & & $90-120 \%$ & \\
\hline $\begin{array}{l}\mathrm{ILY}-\mathrm{FI}-801 \text { (FI-701-A-3) } \\
3 \mathrm{~A}-\mathrm{CMP}-801 \text { SYS GLY FL IND }\end{array}$ & $3 / 11 / 97$ & .733 & & $867, \mathrm{G}$ & \\
\hline $\begin{array}{l}\text { SA-PISL- } 801 \text { (PI } 701 \text { A } 1) \\
\text { SA HDR LO PRESS ALARI }\end{array}$ & $3 / 11 / 97$ & 0734 & & $90-100 . \mathrm{PSI}$ & \\
\hline $\begin{array}{l}\text { SA-TI-805 (TI-701-A-5) } \\
\text { SERVICE AIR HDR TEMP. }\end{array}$ & $3 / 11 / 17$ & 0734 & 6 & $90-120 \%$ & \\
\hline $\begin{array}{l}\text { JUTY CYCLE } \\
\text { (LOAD TIME/TOTAL TIME) }\end{array}$ & $3 / 11 / 77$ & 0730 & $36 \sec 84$ & 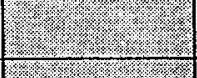 & \\
\hline BELATIVE HUMIDITY & $3 / 4 / 97$ & 10700.8000 & $\frac{95 \%}{29.49}$ & 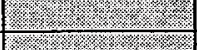 & \\
\hline TTMOSPHERIC PRESSURE , /: & $3 / 11 / 97$ & Jozr. Recording & & & Dis \\
\hline INSIDE TEMP. (IN T01-A) & $3 / 11 / 97$ & 0734 & -68 & & Don \\
\hline $\begin{array}{l}\text { JUTSIDE TEMP } \\
\text { (OUTSIDE } 701-\mathrm{A} \text { ) }\end{array}$ & $3 / 1197$ & 0739 & & & \\
\hline
\end{tabular}

* Call weather station for required information at 373-2875

Note: If readings are outside designated ranges, Notify Test Director.

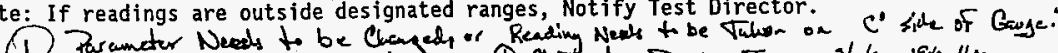

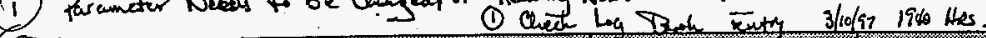

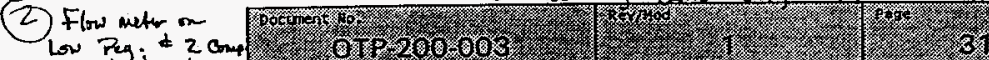

Flow actor stowng to Grm

Band Flow metri. 
HNF-SD-WM-OTR-231, Rev 0

Page 101 of 165

\section{8-HOUR ON-LINE TEST DATA SHEET (Cont.)}

IR AIR DRYER/COOLING SKID

\begin{tabular}{|c|c|c|c|c|c|}
\hline PARAMETER & DATE & ML & READING & EXPECTED RANGE & INIT \\
\hline GY POMP RUNNING & $3-119$ & $\frac{0734}{0730}$ & 80 & 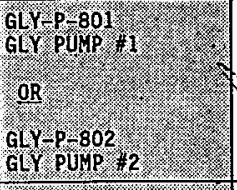 & Men \\
\hline $\begin{array}{l}\mathrm{GLYPI} 801 \text { (PI-701 } \\
\mathrm{GYY} \text { R SUCT PRESS }\end{array}$ & $3 / 11 / 97$ & 0734 & 6.5 & $0-10 \mathrm{pSI}$ & Da \\
\hline $\begin{array}{l}\mathrm{GHY}-\mathrm{PI}-803(\mathrm{PI} 701-\mathrm{A}-16) \\
\mathrm{GEY} \text { P DISCH PRESS }\end{array}$ & $3 / 11 / 47$ & .735 & & 65-75:PSI & \\
\hline $\begin{array}{l}\text { GVY TI } 805 \text { (TI } 701-A=13) \\
\text { GV CIRC PUMP SUCT TEHP }\end{array}$ & $3 / 11 / 97$ & 0735 & 93 & $100 \div 120.1$ & \\
\hline 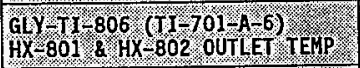 & $3 / 11 / 97$ & 0735 & 91.0 & $100 \div 120 \%$ & $D_{n}$ \\
\hline $\begin{array}{l}\left.\text { GG Y LG } 801 \text { (LGL } 701-\mathrm{A}_{-3}\right) \\
\text { EXPANSION })\end{array}$ & $3 / 11 / 97$ & 0735 & (1) & $1 / 3 \div 2 / 3.1$ & \\
\hline $\begin{array}{l}\text { IA } \triangle \text { PI } 819 \% \\
\text { A TWR } 801 \text { PRESS }\end{array}$ & $3 / 11 / 57$ & 0736 & & 80 & \\
\hline IA PI I 20 PURGE PRES & $3 / 11 / 97$ & 0736 & & 40 $60 . P S I$ & \\
\hline $\begin{array}{l}\text { IAPI } 818 \\
\text { MATHR } 802 \text { PRESS }\end{array}$ & $3 / 11 / 97$ & 0736 & & $80-105$ & \\
\hline 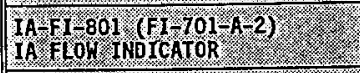 & $3 / 11 / 97$ & 0736 & & $45.90 \mathrm{crm}$ & \\
\hline $\begin{array}{l}\text { IAPI } 821 \text { (PI 701 A } 12) \\
\text { IA }\end{array}$ & $3 / 1157$ & $073 n$ & 42 & 30-45. PST & \\
\hline 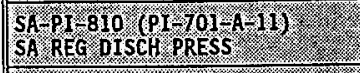 & $3 / 11 / 97$ & 0717 & to & & \\
\hline 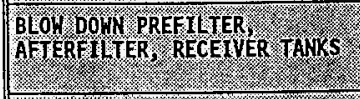 & $3 / 16$ (92 & 0738 & & & \\
\hline 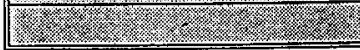 & & & & (1) & \\
\hline
\end{tabular}

(1) Parameter Need to be Changer.

(2) Flow moter an Low By, Possible Bine flow meter. 
HNF-SD-WM-OTR-231, Rev 0

Page 102 of 165

\section{8-HOUR ON-LINE TEST DATA SHEET}

FOR SA-CMP-801

\begin{tabular}{|c|c|c|c|c|c|}
\hline ARAMETER & DATE. & TINE & READING & EXPECTED RANGE & INIT \\
\hline 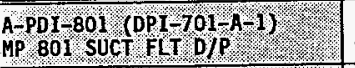 & $3 / 11 / 97$ & 1128 & 1.9 & K. PSI. & \\
\hline $\begin{array}{l}\text { A-PI-801 (PI-701-A-2) } \\
\text { EMOIE BRG 0IL PRESS }\end{array}$ & $3 / 11 / 97$ & 1128 & $\mathbf{Z l}$ & $15-1965$ P & \\
\hline $\begin{array}{l}\text { A-PI } 805 \text { (PI } 701 \text { A-1) } \\
\text { NTER STG DISCH PRESS }\end{array}$ & $3 / 1197$ & 1128 & 26 & $20=40 \mathrm{PST}$ & Dh \\
\hline $\begin{array}{l}\mathrm{LY} F \mathrm{G}-802 \text { (SFI-701 A-4) } \\
\mathrm{A}-\mathrm{CHP}-801 \text { HP SIDE GLY FLO }\end{array}$ & $3 / 11$ & 1128 & & Flow & \\
\hline $\begin{array}{l}\text { A-PI-803 (PI-701-A-4) } \\
\text { MP } 801 \text { DISCH PRESS }\end{array}$ & $3 / 11$ & 1128 & & 85-105.PSI & yh \\
\hline $\begin{array}{l}\text { :A-T 1-BOI (TI-701-A-2) } \\
\text { MP } 801 \text { SA DISCH TEHP. }\end{array}$ & $3 / 1$ & 1128 & & $90-120 \%$ & \\
\hline $\begin{array}{l}3 \mathrm{~A}-\mathrm{SG}-801 \\
; \mathrm{A} \text { CHP } 0 \mathrm{IL} \text { LVL }\end{array}$ & $3 / 11 / 99$ & 1129 & & ABOVE MAP & \\
\hline 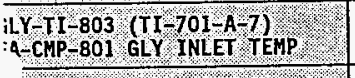 & $3 / 1197$ & 1129 & 103 & $90=120 \mathrm{~F}$ & Dr \\
\hline $\begin{array}{l}\text { LY-TI-801 (II-701-A -9) } \\
3 A-C M P-801 \text { GLY DISCH TEMP }\end{array}$ & $9 \sqrt[11]{1197}$ & 1129 & 10 & 90.120. & \\
\hline $\begin{array}{l}\mathrm{iLY} / \mathrm{FI}-801 \text { (FI-701-A-3) } \\
3 \mathrm{~A}-\mathrm{CHP}-801 \text { SYS GLY FLO IND }\end{array}$ & 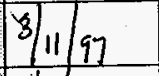 & 1129 & & $367.6 P H$ & \\
\hline $\begin{array}{l}\text { SA-PISL-801 (PI-701-A-1) } \\
\text { IA HDR LO PRESS ALARH }\end{array}$ & $3 / 1197$ & 1130 & 100 & $90 \% 100 \mathrm{PSI}$ & \\
\hline $\begin{array}{l}\text { iA-TL-BO5 (TI-701-A-5) } \\
\text { iERVICE AIR HDR TEMP }\end{array}$ & $3 / 11 / 97$ & 1129 & 102 & $90-120 \%$ & Dun \\
\hline $\begin{array}{l}\text { JUTY CYCLE } \\
\text { (LOAD TME TOTAL, TIHE) }\end{array}$ & $3 / 11 / 97$ & 1127 & 37 sen & & \\
\hline PELATIVE HUMIDITY & $3 / 1197$ & $N / A$ & $N / A$ & & $D_{n}$ \\
\hline ITHOSPHERIC PRESSURE 6 . & $3 / 11 / 97$ & $M / *$ & $N / r$ & 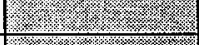 & Dn \\
\hline INSIDE TEMP (IN $701-\mathrm{A})$ & $3 / 11 / 97$ & 1148 & 66 & 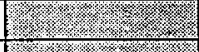 & $D_{n}$ \\
\hline $\begin{array}{l}\text { JUTSIDE TEMP. } \\
\text { (OUTSIDE 7OI-A) }\end{array}$ & $3 / 11 / 97$ & $1 / 48$ & 48 & & D \\
\hline
\end{tabular}

* Call weather station for required information at $373-2875$

Note: If readings are outside designated ranges, Notify Test Director.

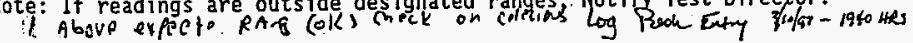

(2) (ta) Guige 


\section{8-HOUR ON-LINE TEST DATA SHEET (Cont.)}

JR AIR DRYER/COOLING SKID

\begin{tabular}{|c|c|c|c|c|c|}
\hline PARAMETER & GATE. & TIME & REAOING & EXPECTED RANGE & $1 \mathrm{MIT}$ \\
\hline 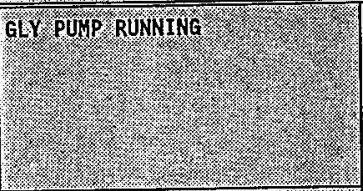 & $3 / 11 / 57$ & $1130^{\circ}$ & 802 & 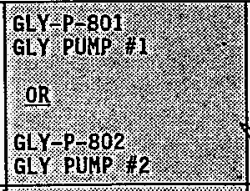 & $\eta_{u}$ \\
\hline 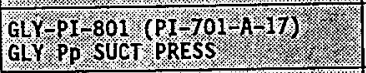 & $3 / 11 / 19$ & 1136 & & $0.10 \mathrm{ps}$ & $D_{4}$ \\
\hline $\begin{array}{l}\text { GYYPI } 803\left(\text { PI } 701-{ }^{A}-16\right) \\
\text { GLY PD DISCH PRESS }\end{array}$ & $3 / 1 / 17$ & 1130 & 63 & 65:75.PSI & $\mathrm{P} / 4$ \\
\hline $\begin{array}{l}\text { GLY TI } 805 \text { (TI-701 A } 13) \\
\text { GLY CIRC PUHP SUCT TETP }\end{array}$ & $3 / 11 / 17$ & 1130 & 103 & $100-120 \cdot \mathrm{F}$ & $D_{4}$ \\
\hline 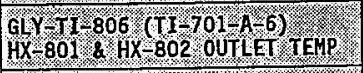 & $3 / 11 / 97$ & 1131 & 105 & $100-120$ & 8 \\
\hline 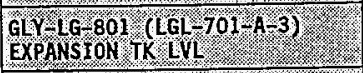 & $3 / 119$ & 1131 & $3 / 4$ & $1 / 3-2 / 3$ ru & $9 / 4$ \\
\hline $\begin{array}{l}\text { IA }=81819 \\
\text { A TWR } 801 \text { PRESS }\end{array}$ & 8/11 & 1132 & 105 & $80 \% 105$ PSI & $D_{n}$ \\
\hline $\begin{array}{l}\text { IA P I } 820 \text {. } \\
\text { IA PURGE PRESS }\end{array}$ & $3 / 1197$ & 1132 & 45 & $40 \% 60 \mathrm{PSI}$ & $\nabla_{4}$ \\
\hline $\begin{array}{l}\text { IA P I } 818 \text {, } \\
\text { IA TWR } 802 \text { PRES }\end{array}$ & $5 / 1 1 \longdiv { 1 7 }$ & 1132 & $\not$ & 60\% $105 . \mathrm{pS}$ & 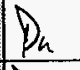 \\
\hline 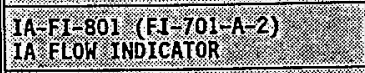 & Blilas & 1132 & 20 & $45-90$ CEM & D. \\
\hline 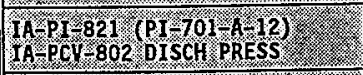 & $3 / 11 / 97$ & 1132 & 42 & 30-45. PSI & $D$ \\
\hline $\begin{array}{l}\text { SAMP } 810 \text { (PI } 701-A=11) \\
\text { SA.REG DISCH RRES }\end{array}$ & $3 / 1199$ & 1132 & 40 & 25-45 PST & $D_{4}$ \\
\hline $\begin{array}{l}\text { BLOW DOWH PREFILTER, } \\
\text { AFTERTIGER, RECETYER TARS }\end{array}$ & 31199 & & & & $D$ \\
\hline l( & & & & & \\
\hline
\end{tabular}

(i) Tas Frov Indiente. 
HNF-SD-WM-OTR-231, Rev 0

Page 104 of 165

WORKING COPY

1.

\section{8-HOUR ON-LINE TEST DATA SHEET}

FOR SA-CMP-BO1

\begin{tabular}{|c|c|c|c|c|c|}
\hline PARAMEIER & DATE & TINE & READING & EXPECTEO RANGE & INI \\
\hline $\begin{array}{l}\text { SA-PDI-BO1 (DPI-701/P-1) } \\
\text { CHP 801 SUCT FLT D/P }\end{array}$ & $3 / 11 / 97$ & 1531 & 1.9 & Kr psi & \\
\hline $\begin{array}{l}\text { SA-PI-801 ( PI-701-A-2) } \\
\text { REMOTE BRG OIL PRESS }\end{array}$ & $3 / 11 / 97$ & 1531 & 21 & $15=66 \%$ & \\
\hline $\begin{array}{l}\text { SA-PI-805 (PI-701-A-1) } \\
\text { INTER STG DISCH PRESS }\end{array}$ & $3 / 11 / 47$ & 1532 & 27 & $20-40 \mathrm{pST}$ & \\
\hline $\begin{array}{l}\text { GLY-FG-802 (SFI-701-A-4) } \\
\text { SA-CHP-801 HP SIDE GLY FLO. }\end{array}$ & $3 / 1$ & 1533 & & FLOH & \\
\hline $\begin{array}{l}\text { SA-PI-803 (PI-701-A-4) } \\
\text { CMP } 801 \text { DISCH PRESS }\end{array}$ & $8 / 11 / 97$ & 1533 & & $85-105$ pst & \\
\hline $\begin{array}{l}\text { SA-TI-801 (TI-701-A-2) } \\
\text { CMP } 801 \text { SA DISCH TERP }\end{array}$ & & 1533 & & $90-120 . \mathrm{F}$ & \\
\hline $\begin{array}{l}\text { SA-SG-801 } \\
\text { SA CHP 0IL LVL. }\end{array}$ & & 1534 & & ABOVE MA & \\
\hline $\begin{array}{l}\mathrm{GLY}-\mathrm{TI}-803 \text { (TI-701-A-7) } \\
\mathrm{C4}-\mathrm{CMP}-801 \text { GLY INLE) TEMP. }\end{array}$ & 3 & 1534 & 103 & $90-120 . \mathrm{F}$ & \\
\hline $\begin{array}{l}\text { LLY-TI-801 (TI-701-A-9) } \\
\text { SA-CMP-801 GLY DISCH TEMP. }\end{array}$ & $3 / 11 / 9$ & 1534 & & $90-120 \%$ & \\
\hline $\begin{array}{l}\text { GLY-FI-BO1 (FI-701-A-3) } \\
\text { SA-CMP-801 SYS GLY FLO IND }\end{array}$ & $3 / 1$ & 1534 & & K67. GPH & \\
\hline $\begin{array}{l}\text { SA-PISL-801 (PI-701-A-1) } \\
\text { SA HDR LO PRESS ALARM }\end{array}$ & 3 & 1534 & 99 & $90-100$ PSI & \\
\hline $\begin{array}{l}\text { SA-TI-805 (TI-70I A-5) } \\
\text { SERVICE AIR HDR TEMP }\end{array}$ & 1 & 1531 & 104 & $90-120$ & \\
\hline $\begin{array}{l}\text { DUTY CYCLE } \\
\text { (LOAD TIME/TOTAL TIME) }\end{array}$ & $3 / 11 / 97$ & 1530 & 3 & & \\
\hline RELATIVE HUMIDITY & & 1528 & & & \\
\hline ATMOSPHERIC PRESSURE & $3 / 11 / 97$ & 1528 & 3:000 R Ren & & \\
\hline INSIDE TEMP, $(I N, 01 / \mathrm{A})$ & $3 / 11 / 97$ & 1532 & 76 & & \\
\hline $\begin{array}{l}\text { OUTSIDE TEMP. } \\
\text { (OUTSIDE } 701-\mathrm{A} \text { ) }\end{array}$ & $\sqrt[3]{11 / 97}$ & 1528 & $\begin{array}{r}51 \\
3: 00 \mathrm{pm} \\
\end{array}$ & 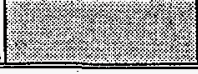 & \\
\hline
\end{tabular}

* Call weather station for required information at 373-2875

Note: If readings are outside designated ranges, Notify Test Director.

(1) Bail Flow Meter

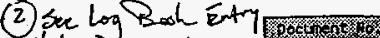
$3 / 6197$ 1940 thes. 
HNF-SD-WM-OTR-231, Rev 0

Page 105 of 165

\section{WORKING COPY}

7.

\section{8-HOUR ON-LINE TEST DATA SHEET}

FOR SA-CHP-801

\begin{tabular}{|c|c|c|c|c|c|}
\hline ARAMEIER & OATE & $M W E$ & READVIS & EXPEOLO YRAYGE & $1 \mathrm{INI}$ \\
\hline 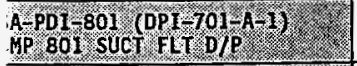 & $3-11-97$ & 2830 & 1 & 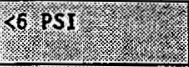 & $\pi$ \\
\hline 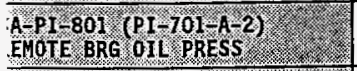 & & 2330 & 26 & $15 \times 40$ & $\pi \mathrm{C}$ \\
\hline 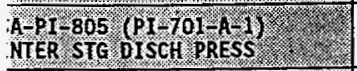 & & & 26 & $20=40 \%$ & IIC \\
\hline 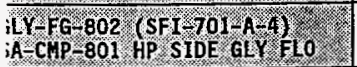 & & 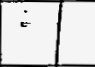 & & ELOH & Tre \\
\hline 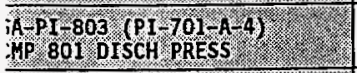 & & & & 85.105. & D \\
\hline 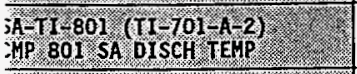 & & $\because:$ & 180 & $90=120$ & $\pi c$ \\
\hline $\begin{array}{l}\text { iASG } 801 \\
\text { IA CHP OH }\end{array}$ & & & & ABOY & inc \\
\hline 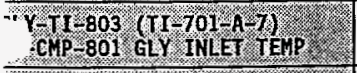 & & & & $90=120.9$ & \\
\hline 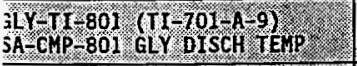 & & & & $90-120$ & \\
\hline 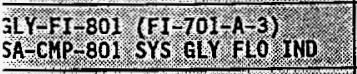 & & & (2) & 667 67 & $7 x$ \\
\hline 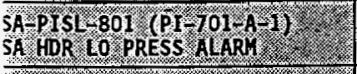 & & & & $900100 . \mathrm{PSI}$ & \\
\hline 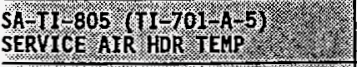 & & & -0 & $90.120 \%$ & $\pi$ \\
\hline DUTY CYCLE & & & & & Tk \\
\hline RELAT VE GUNIDIV & & & & & \\
\hline ATHOSPHERTC RRESSURE (1) & & & & & \\
\hline 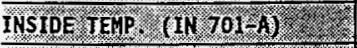 & & , & 69 & & $\pi$ \\
\hline $\begin{array}{l}\text { OUTSIDE TIMP } \\
\text { (OUTSIDE } 701 \text { ) }\end{array}$ & U & $V$ & & 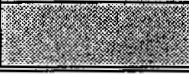 & $m 6$ \\
\hline
\end{tabular}

- Call weather station for required information at 373-2875

.ote: If readings are outside designated ranges, Notify Test Director.

(i) See $\log$ Book entry $\$ / 10 / 97-19$ th thes.

(2.) Bach frow mato.

ved 1802 
HNF-SD-WM-OTR-231, Rev 0

Page 106 of 165

WORKING COPY

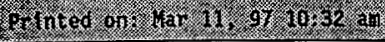

48-HOUR ON-LINE TEST DATA SHEET (Cont.)

FOR AIR DRYER/COOLING SKID

\begin{tabular}{|c|c|c|c|c|c|}
\hline PARANETER & DATE & HII & READING & EXPECTEO RAYGE & INIT \\
\hline 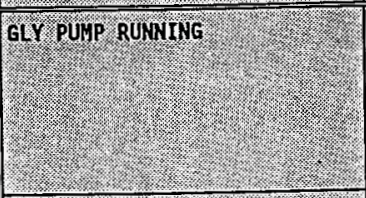 & $3-11-87$ & 2330 & & 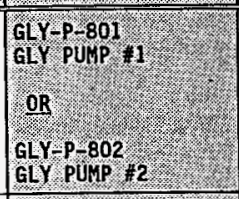 & $\Pi K$ \\
\hline $\begin{array}{l}\text { GLY PI I } 801 \text { (PI } 701 \text { - }-17) \\
\text { GLY Pp SUCT PRESS }\end{array}$ & & & 6 & $0-10$ PST & $T K C$ \\
\hline 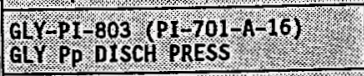 & & & 58 & $65-75$ PSI & The \\
\hline 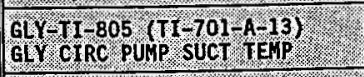 & & & 94 & $100-120 \%$ & ths \\
\hline 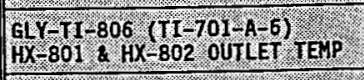 & & & 96 & $100-120 \mathrm{~F}$ & the \\
\hline 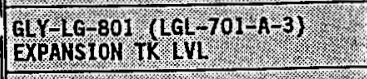 & & & $3 / 4$ & $1 / 3-2 / 3$ rwit & the \\
\hline $\begin{array}{l}\text { IA P I } 819 \\
\text { IA THR } 801 \text { PRESS }\end{array}$ & & & 0 & $80-105$ PSI & $\pi$ \\
\hline $\begin{array}{l}\text { IA PI B } 20 \\
\text { IA PURGE PRESS }\end{array}$ & & & 45 & $40 \div 60 \mathrm{PST}$ & te \\
\hline $\begin{array}{l}\text { TA-PI } 818 \\
\text { IA THR } 802 \text { PRESS }\end{array}$ & & & & $80-105$ psi & TK \\
\hline 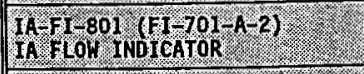 & & & & $45-90 \mathrm{cFH}$ & $\pi x$ \\
\hline 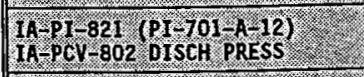 & & & 40 & $30-45$ psi & $7 x$ \\
\hline SA PT 810 (P T TOI A -11$)$ & & & 39 & $25-45.851$ & 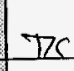 \\
\hline 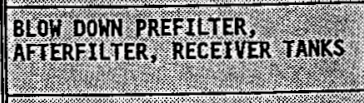 & & & & & Tx \\
\hline 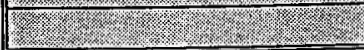 & $l$ & l & & 1.2 .0 & \\
\hline
\end{tabular}

(1) Low Air Usage. 
HNF-SD-WM-OTR-231, Rev 0

Page 107 of 165

\section{WORKINE COPY}

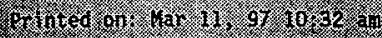

\section{8-HOUR ON-LINE TEST DATA SHEET}

\begin{tabular}{|c|c|c|c|c|c|}
\hline ARAMEER. & $\mathrm{BATE}$ & TISE & READING & EXPECTED RANGE & 111 \\
\hline 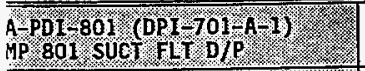 & $3-12-9 ?$ & 6330 & 1. & K6.9. & K \\
\hline 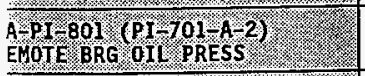 & & & & 15.48.65.PSI & $T 2$ \\
\hline 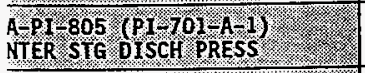 & & & & $20-40 \times 15$ I & $T C$ \\
\hline 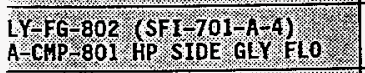 & & $\therefore$ & & $100 \mathrm{~W}$ & $\pi c$ \\
\hline 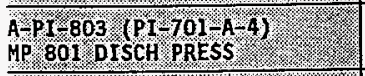 & & & & 85.105. & $\pi c$ \\
\hline 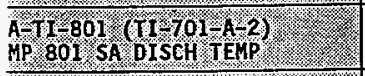 & & $\because=$ & (1): & $90-120$ & $T K$ \\
\hline $\begin{array}{l}\text { A. SG } 801 \\
\text { A CCIP } 01 \text { L LV. }\end{array}$ & & & & ABOYEYA & - \\
\hline 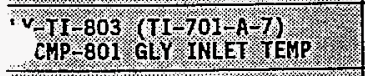 & & & & $90120 \%$ & $T K$ \\
\hline 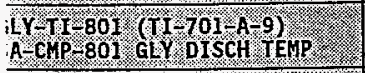 & & & & $90=120$ & $\pi c$ \\
\hline 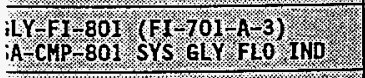 & & & (2) & $67 . \mathrm{GPH}$ & PC \\
\hline 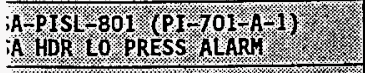 & & & & $90.100 . \mathrm{PS}$ & $T K$ \\
\hline 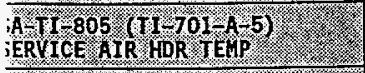 & & & 01 & $90-120.9$ & $\pi$ \\
\hline 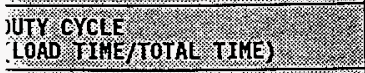 & & & $\begin{array}{l}45 \\
32 / 78 \\
\end{array}$ & 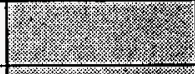 & $\pi K$ \\
\hline ELTIUE HUMIDIY & & & $N / A$ & 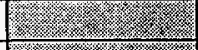 & TK \\
\hline THOSPHERTC PRESSURE. / / / & & . & $N / A$ & & $x$ \\
\hline 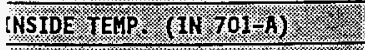 & & & 67 & & $\pi k$ \\
\hline $\begin{array}{l}\text { JUTSTEE TEMP } \\
\text { OUTSIDE } 701 \% \text { ) }\end{array}$ & & & 3 & & $T$ \\
\hline
\end{tabular}

- Call weather station for required information at $373-2875$

ste: If readings are outside designated ranges, Notify Test Director.

(1) Seelog BodK Caty 3-10.57 1940 Hes.

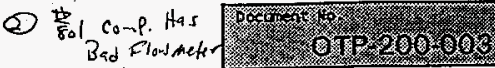
used gor


HNF-SD-WM-OTR-231, Rev 0

Page 108 of 165

\section{WORKING COPY}

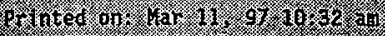

\section{8-HOUR ON-LINE TEST DATA SHEET (cont.)}

FOR AIR DRYER/COOLING SKID

\begin{tabular}{|c|c|c|c|c|c|c|}
\hline PARAMETER & DATE & $\mathrm{WIJE}$ & & REAOMG & EXECTED RANGE & MI \\
\hline 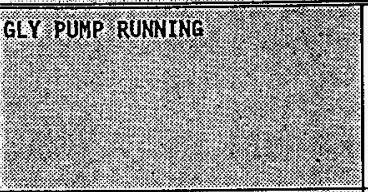 & $3^{-12-91}$ & 0330 & & & 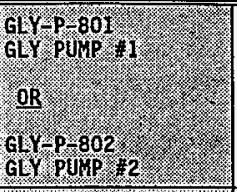 & $7 \mathrm{TC}$ \\
\hline 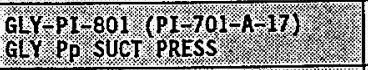 & & $=$ & & & 0-10:PSI & $\pi c$ \\
\hline 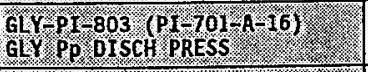 & & & & & 65.75 & $7 c$ \\
\hline 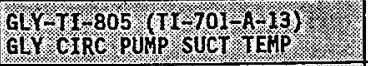 & & & & & 100 & c \\
\hline 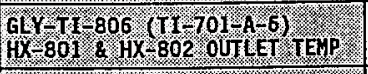 & & & . & & $100-120$ & k \\
\hline 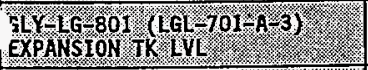 & & & & & & $D C$ \\
\hline IA PI 819 . & & & & & 80.905 .951 & \\
\hline $\begin{array}{l}\text { A P P } 820 \\
\text { A PURGE PRESS }\end{array}$ & & & & & $40 \mathrm{sog}$ pS & $D$ \\
\hline IA PI 818 . & & & & & $80 \% 05$ PSP & $D K$ \\
\hline 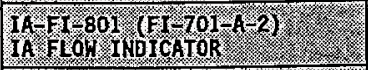 & & & & & $45=90 \mathrm{CF}$ & \\
\hline 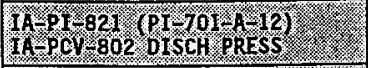 & & & & & 309 45.95 & \\
\hline 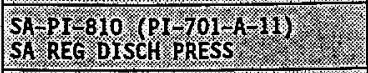 & & & & & 2545 p p & Tr \\
\hline 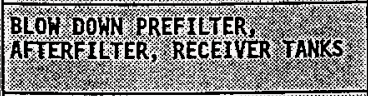 & : & & & & 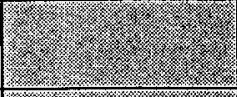 & $\pi x$ \\
\hline 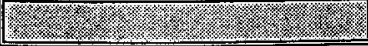 & 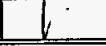 & 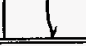 & & & 1.8x & . \\
\hline
\end{tabular}

(1) Notified test director. Consistent w/previous reodings. 
HNF-SD-WM-OTR-231, Rev 0

Page 109 of 165

\section{WORKINE.COPY}

4.

\section{8-HOUR ON-LINE TEST. DATA SHEET}

\begin{tabular}{|c|c|c|c|c|c|}
\hline PARAMETER & DATE & TIWE & READING & EXPECTED RAKGE & \\
\hline 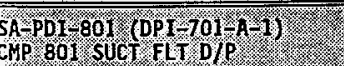 & $3 / 12 / 97$ & 072750 & 2.0 & K. PST & \\
\hline 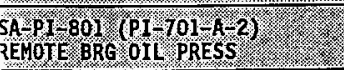 & $3 / 12 / 97$ & 0729 & 22 & $15 \%$ & \\
\hline $\begin{array}{l}\text { SA PI } 805 \text { (PI 701 A } 11 \text { ) } \\
\text { INTER STG DISCH PRESS }\end{array}$ & & $0130=$ & 26 & $20=40.95$ & \\
\hline 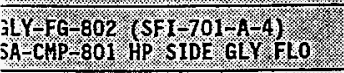 & $8 / 12 / 47$ & $\therefore 0730$ & & FLoN & \\
\hline $\begin{array}{l}\text { SA-PI 803 }(\mathrm{PI} 701-\mathrm{A}-4) \\
\mathrm{ZMP} 801 \text { DISCH PRESS }\end{array}$ & $3 / 12 / 91$ & 0731 & & 85. $105 \%$ & \\
\hline 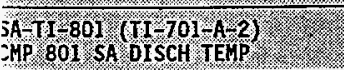 & $3 / 12$ & $=8731$ & $176^{1}$ & $90+120 \%$ & \\
\hline 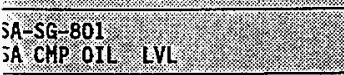 & $3 / 12$ & 0731 & & ABOVE MAP & \\
\hline 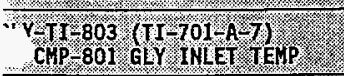 & $3 / 12 / 97$ & 1732 & & 90.120 .1 & \\
\hline 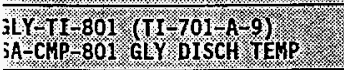 & $3 / 12 / 67$ & .732 & & 90.120 .7 & \\
\hline 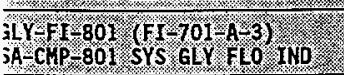 & $3 / 12 / 19$ & 0732 & & $\mathrm{~K} 6 \mathrm{GPH}$ & \\
\hline 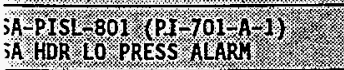 & $3 / 12 / 97$ & 0733 & 9 & $90-100 \%$ & \\
\hline 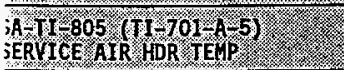 & $3 / 12 / 17$ & 0729 & 81 & $90-20 \%$ & \\
\hline 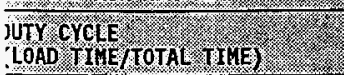 & $3 / 12 / 47$ & .0128 & $31_{\text {sec }} / 93$ sec. & & \\
\hline 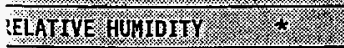 & $3 / 12 / 17$ & 0738 & niso & & $\sim$ \\
\hline ITHOSPHERIC PRESSURE & $\sqrt[3]{12} 19$ & 0.734 & $\log$ Recortins & & \\
\hline NSIDE $/ E M P$ (N $(N 01-A)$ & $3 / 12 / 47$ & 8728 & 66 & & Dn \\
\hline 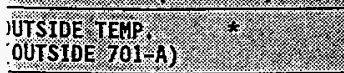 & $3 / 12 / 19$ & 0738 & $\begin{array}{c}\text { oxo Rewhling } \\
40\end{array}$ & & D \\
\hline
\end{tabular}

- Call weather station for required information at 373-2875

.ote: If readings are outside designated ranges, Notify Test Director.

(1) see $\log$ bouk Enty 1940 HRS 3-10-92

(2) Dad Flow rett 
HNF-SD-WM-OTR-231, Rev 0

Page 110 of 165

\section{WORKING COPY}

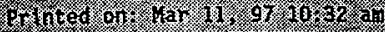

48-HOUR ON-LINE TEST DATA SHEET (Cont.)

FOR AIR DRYER/COOLING SKID

\begin{tabular}{|c|c|c|c|c|c|}
\hline PARAHETER : & DATE & TIVE: & REAOING & EXPECTED RANGE & $1 N 1$ \\
\hline 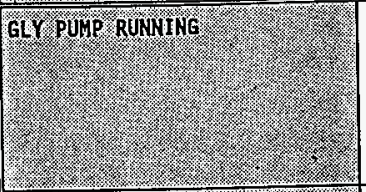 & $3 / 1261$ & 1733 & 802 & 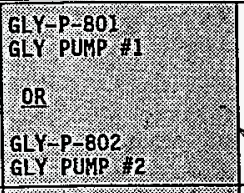 & \\
\hline 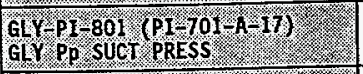 & $3 / 2 / 47$ & 0733 & 2.5 & 0-10. PSI & \\
\hline 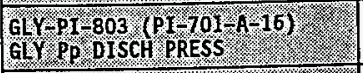 & & 0733 & 56 & $65-7$ PST & \\
\hline 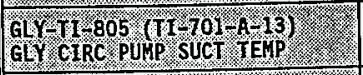 & & b733 & 78 & $100-120 \%$ & \\
\hline 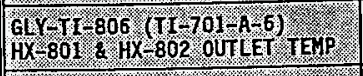 & & 0234 & 79 & $100-120 \%$ & \\
\hline 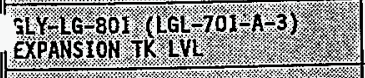 & & 0734 & $3 / 4$ & $1 / 3-2 / 3 \cdot 1$ & \\
\hline $\begin{array}{l}\text { IA PI - B 19 } \\
\text { IA IIR } 801 \text { PRESS }\end{array}$ & $3 / 12 / 97$ & 1734 & & 80-105. PS1 & \\
\hline $\begin{array}{l}\text { IA PIIGLO } \\
\text { IA PURGE PRES }\end{array}$ & $3 / \sqrt{2 / 47}$ & 0734 & & $40-60.95$ & \\
\hline $\begin{array}{l}\text { IA PI } 818 \text {, } \\
\text { IA } 14 R \text { RO 2 RRESS }\end{array}$ & $3 / 1 / 2 / 27$ & $0734^{\circ}$ & & 80-105. PS & \\
\hline 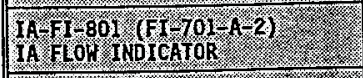 & $3 / 12 / 97$ & 0734 & 20 & 45-90 cFX & \\
\hline 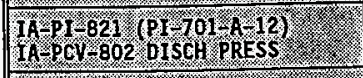 & $3 / 12 / 47$ & 0735 & 1 & 30-45. .01 & \\
\hline 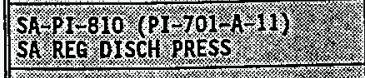 & $9 / 12 / 97$ & 0735 & 39 & $25=45$. PSI & \\
\hline $\begin{array}{l}\text { BLOA DOWN PRET TLER, } \\
\text { AFIERFILTER, RECEMER TAMS }\end{array}$ & $\sqrt[3]{12 / 97}$ & .735 & & & \\
\hline 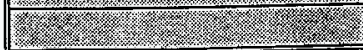 & & & & in & \\
\hline
\end{tabular}

(1) Liv Air Usage 
HNF-SD-WM-OTR-231, Rev 0

Page 111 of 165

\section{8-HOUR ON-LINE TEST DATA SHEET}

FOR SA-CHP-801

\begin{tabular}{|c|c|c|c|c|c|}
\hline ARAHETER & BATE & The - & REAOHG & EXPEOTED RANGE & 1N1 : \\
\hline 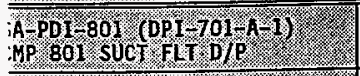 & $3 / 12 / 11$ & 1129 & 1.9 & K PST & Dh \\
\hline 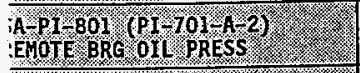 & $3 / 12 / 92$ & 1129 & 25 & $15-6 \%$ & \\
\hline 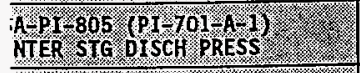 & $\sqrt[3]{12 / 97}$ & 1130 & 25 & $20=40.95$ & h \\
\hline 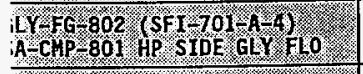 & & $\therefore \quad 1130$ & & $100 \%$ & Dn \\
\hline $\begin{array}{l}\text { YAPI } 803 \text { (PI-701-A } 4) \\
\text { HP } 801 \text { DISCH PRESS }\end{array}$ & $3 / 196$ & 1130 & 94 & 85.105 .9 & 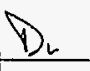 \\
\hline A T 801 S SA DISCH TEHP & & $=1130$ & $\because 180$ & $90-120$. & \\
\hline 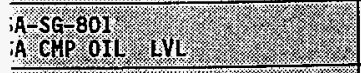 & & 1131 & & ABOVE MA & . \\
\hline 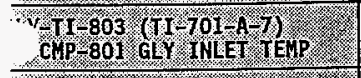 & & || $3 \mid$ & 88 & $90-120.9$ & 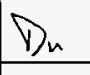 \\
\hline 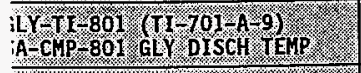 & $3 / 1$ & 1131 & 87 & $90=120 \%$ & \\
\hline 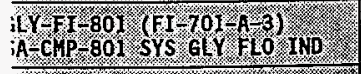 & $3 / 12$ & 1132 & (2) & \%67. GPH & \\
\hline 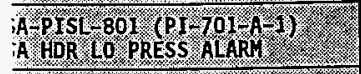 & $3 / 1$ & 1132 & & 90.100 .951 & \\
\hline 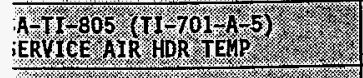 & $3 / 1$ & 1129 & LT & $90-120.7$ & \\
\hline 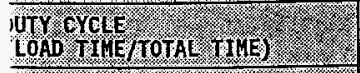 & $3 / 1$ & 1128 & $3530 / 8$ & & \\
\hline ELATUE HUTIDIY & $5 / 12,97$ & & & & \\
\hline THOSPHERIC PRESSURE > & $3 / 12 / 97$ & & $N / A$ & (x) & Dn \\
\hline NSIDE TEMP ( 1 \% $01 \%$ A) & $3 / 12 / 97$ & 1146 & 68 & & Dn \\
\hline 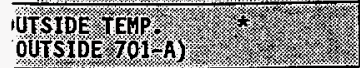 & $3 / 12 / 97$ & Nise & 3 & & $D_{1}$ \\
\hline
\end{tabular}

Call weather station for required information at 373-2875

.ute: If readings are outside designated ranges, Notify Test Director.

(1) Sue log Book Eentry B/rgar 1940 HAs.

B Bad Fiow meter 
HNF-SD-WM-OTK-231, Rev 0

Page 112 of 165

\section{WORKING COPY}

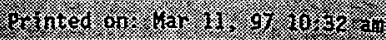

48-HOUR ON-LINE TEST DATA SHEET (Cont.)

FOR AIR DRYER/COOLING SKID

\begin{tabular}{|c|c|c|c|c|c|}
\hline BARAMETER & DATE: & IILE & READ N N & EXPECTED RA & nin \\
\hline 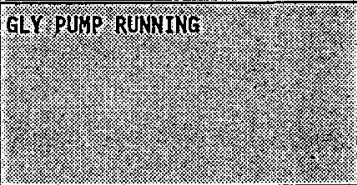 & $3 / 1 / 91$ & 1132 & 802 & 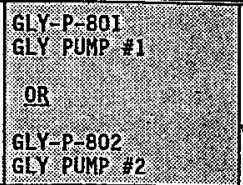 & $\lambda_{n}$ \\
\hline $\begin{array}{l}\text { GLY I I RO (PI } 701 \text { A } 17) \\
\text { GU PP SUT PRESS }\end{array}$ & $3 / 12 / 99$ & 1133 & 4 & 0.10 PSI & $D_{n}$ \\
\hline 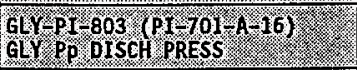 & $3 / 12 / 97$ & 1133 & 57 & $65-75$ PSI. & $D_{n}$ \\
\hline 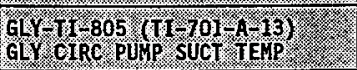 & $3 / 12 / 99$ & 1133 & 88 & $100-120 \%$ & a \\
\hline 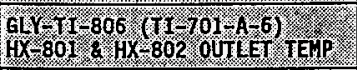 & $3 / 12 / 97$ & 1133 & 90 & $100-120 \mathrm{~F}$ & h \\
\hline 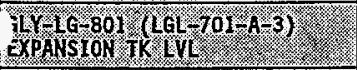 & $2 / n / 91$ & 1133 & 14 & $1 / 3-2 / 3 / \mathrm{FU}$ & \\
\hline 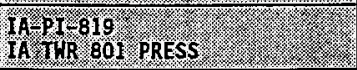 & $312 / 91$ & 1134 & 105 & $80-105$ PST & Dh \\
\hline 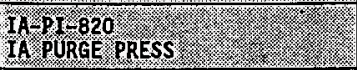 & $3 / 12 / 47$ & 1134 & 45 & & $h$ \\
\hline 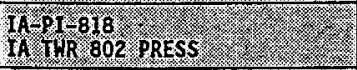 & $3 / 2 / 19$ & 1134 & $\varnothing$ & $80-105$ PSI & $D_{h}$ \\
\hline 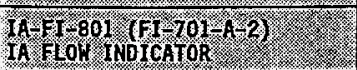 & $3 / 1 / 419$ & 1134 & 20.0 & & $D_{n}$ \\
\hline 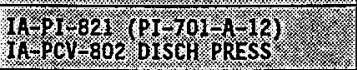 & $3 / 2 / 97$ & 1135 & 41 & 30.45 PSI & $m$ \\
\hline 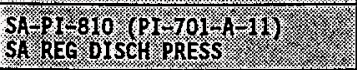 & $3 / 12 / 97$ & 1135 & 39 & 25.45 ? SI & $D_{h}$ \\
\hline 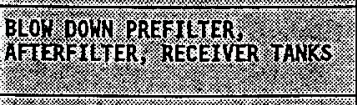 & $3 / 12 / 27$ & 1135 & & & $h$ \\
\hline 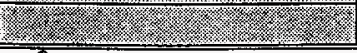 & & & & 12 & \\
\hline
\end{tabular}

(1) Low Air Usage. 
HNF-SD-WM-OTR-231, Rev 0

Page 113 of 165

48-HOUR ON!-LINE TEST DATA SHEET (cont.)

\begin{tabular}{|c|c|c|c|c|c|}
\hline PARAMETER & DATE & TIME & READIHG & EXPECTED RANGE & \\
\hline 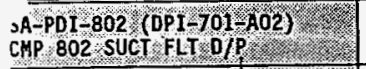 & $3 / 12 / 97$ & 1031 & 2.4 & K6. PS I & Dh \\
\hline $\begin{array}{l}\text { SA-PI-802 (PI-701/A } 6) \\
\text { REMOTE BRG OIL PRESS }\end{array}$ & $\{\operatorname{lig}(9)$ & 1031 & 48 & $15.4 .66 \% \mathrm{pI}$. & an \\
\hline $\begin{array}{l}\text { SA-PI I } 806 \text { (PI-701 A } 5) \\
\text { INTER SIG DISCH PRESS }\end{array}$ & $3 / 17 / 97$ & 1032 & 29 & $20-40$ & \\
\hline 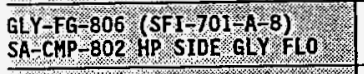 & $3 / 17 / 97$ & $1032^{-}$ & & FloH & \\
\hline $\begin{array}{l}\text { SA-PI-804 (PI } 701 \text { A } 8 \text { ) } \\
\text { CMP } 802 \text { DISCH PRESS }\end{array}$ & $3 / 17 / 97$ & 1032 & 110 & $85-105$ & \\
\hline $\begin{array}{l}\text { SA-TI }-802 \text { (TI } 701-A-4) \\
\text { CMP } 802 \text { SA DISCH TEPP. }\end{array}$ & $3 / 17 / 97$ & 1032 & 100 & $90-120$ & \\
\hline $\begin{array}{l}\mathrm{SA}-\mathrm{SG}-802 \\
\mathrm{SA} \mathrm{CHP} 0 \mathrm{IL} \mathrm{LL}\end{array}$ & $3 / 17 / a 7$ & 1032 & & ABOVE & \\
\hline $\begin{array}{l}\text { GLY-TI-804 (TI } 701-A-10) \\
\text { SA-CHP-802 GLY INLET TEMP }\end{array}$ & $3 / 17 / 97$ & 1033 & 10 & $90-120$ & \\
\hline $\begin{array}{l}\mathrm{GLY} T \mathrm{TI}-802 \text { (T I-701 }-\mathrm{A}-12) \\
\mathrm{SA}-\mathrm{CHP}-802 \mathrm{GL} \mathrm{OTSCH} \mathrm{TEYP}\end{array}$ & $3 / 17 / 97$ & 1033 & 8 & $90 \div 120$ & \\
\hline 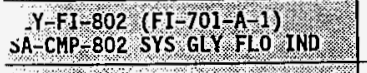 & $3 / 19 / 97$ & 1033 & & $\mathrm{~K} 67 \mathrm{GPH}$ & \\
\hline $\begin{array}{l}\text { SA-PISL-801 (PI-7OI-A- }) \\
\text { SA HDR LO PRESS ALARM }\end{array}$ & $3 \log _{97}$ & 1033 & 100 & $90,100, p$ & \\
\hline $\begin{array}{l}\text { SA-TI- } 805 \text { (TI 701 A } 5 \text { ) } \\
\text { SERVICE AIR HDR TEMP }\end{array}$ & $3 / 12 / 97$ & 1033 & & 90 & \\
\hline $\begin{array}{l}\text { DUTY CYCLE, } \\
\text { (LOAD TIME/TOTAL TIME) }\end{array}$ & $3 / 12 / 97$ & 1038. & $64 \sec$ & & \\
\hline RELATIVE HUMIDIYY $>$, & $3 / 12 / 17$ & 1040 & & & Da \\
\hline ATHOSPHERIC PRESSURE - - & $3 / 10 / 72$ & 1040 & $\Omega_{0}^{21.12}$ Recomiting & 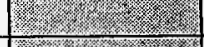 & $D_{4}$ \\
\hline INSIDE TEMP $(\mathrm{IN}, 01 \mathrm{~A})$ ) & $.3 / 17 / 47$ & 1036 & 63 & & $D_{n}$ \\
\hline $\begin{array}{l}\text { OUTSIDE TEMPERATURE } \\
\text { (OUTSIDE } 701-\mathrm{A})\end{array}$ & $3 / 10 / 197$ & -1036 & 5 & & Du \\
\hline
\end{tabular}

* Call weather station for required information at $373-2875$

Note: If readings are outside designated ranges, Notify Test Director. 
48-HOUR ON-LINE TEST DATA SHEET (Cont.)

OR AIR DRYER/COOLING SKID

\begin{tabular}{|c|c|c|c|c|c|}
\hline 2.0 & DATE & WHE & REABING & EXPECT ED RANGE & $1 \mathrm{INI}$ \\
\hline GLP PUNP RUNAING & & 1034 & & 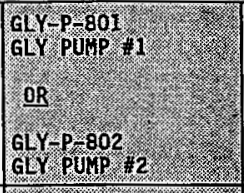 & \\
\hline 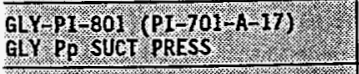 & & 1034 & & 0-10 & \\
\hline 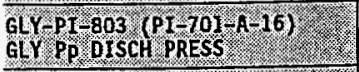 & & .1034 & 61 & 65.75 PSI & \\
\hline 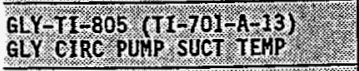 & • & $1034^{\circ}$ & 73 & $100-120 \%$ & \\
\hline 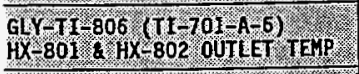 & & 1035 & 17 & $100 \cdot 120 \%$ & \\
\hline 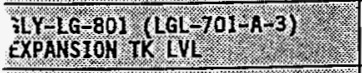 & & 1035 & & $13-213.70$ & \\
\hline $\begin{array}{l}\text { IA PIV } 819 \\
\text { IA TWR } 801 \text {. PRESS }\end{array}$ & & 1035 & & 80. $105 . \mathrm{ps}$ & \\
\hline $\begin{array}{l}\text { IA PIR2O } \\
\text { IA PURGE PRESS }\end{array}$ & & 1035 & & 40. $60.9 S T$ & \\
\hline $\begin{array}{l}\text { IA PI-818 } \\
\text { IA TW } 802 \text { RRES }\end{array}$ & & 1035 & 4 & 80.105.PSI & \\
\hline 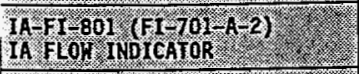 & & 1035 & 20 & $45=90.6$ & \\
\hline 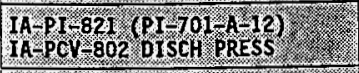 & & 1035 & 43 & $30=45.95$ & \\
\hline 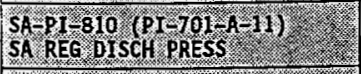 & & 1035 & 41 & $25=45.031$ & \\
\hline 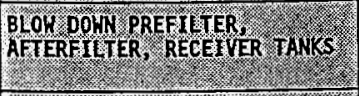 & & 1036 & & & \\
\hline 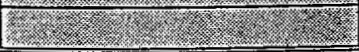 & & & & $\sqrt{2.8}$ & \\
\hline
\end{tabular}


HNF-SD-WM-OTR-231, Rev 0

Page 115 of 165

48-HOUR ON-LINE TEST DATA SHEET [rnnt.)

FOR SA-CMP-802

\begin{tabular}{|c|c|c|c|c|c|}
\hline ARAMETER - & DAIE & IME & READING & EXPECTED & \\
\hline 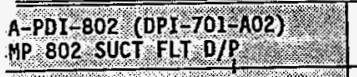 & $3 / \ln 292$ & 1045 & 2 & & Qh \\
\hline $\begin{array}{l}\text { A.PI-802 (PI-701-A } 6 \text { ) } \\
\text { EMOTE BRG OIL PRESS }\end{array}$ & $3 / \ln \lg 7$ & 1045 & 38 & $15-696$ & Dh \\
\hline 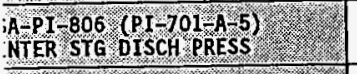 & $3 / 17 / 47$ & 1045 & 29 & $20-40$ & \\
\hline 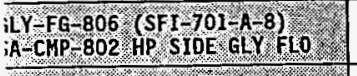 & $3 / 17 / 47$ & 1044 & & & \\
\hline $\begin{array}{l}\text { A-PI- } 804 \text { (PI- 701A-8) } \\
\text { MP BO2 DISCH PRESS }\end{array}$ & $3 / \operatorname{lol}(a)$ & 1046 & 110 & & \\
\hline $\begin{array}{l}\text { A-T T } 802 \text { (TI TO1 A } 4) \\
\text { MP } 802 \text { SA DISCH TEMP }\end{array}$ & $\hat{b} / 17\}_{7}$ & 1046 & (2) & & \\
\hline $\begin{array}{l}3 \mathrm{~A}-\mathrm{SG}=802 \\
3 \mathrm{~A} \text { CHP OIL L VL }\end{array}$ & $z / 17 / 97$ & 1044 & & & \\
\hline 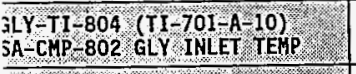 & $2 / 62 / 91$ & 1044 & 72 & & \\
\hline $\begin{array}{l}\text { ILY TI } 802 \text { (TI } 701-A-12) \\
\text { SA COMP } 802 \text { GLY DISCH TEMP }\end{array}$ & $3 / \log / 2$ & $10 \%$ & 73 & & \\
\hline 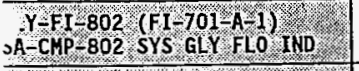 & $3 / 172 / 99$ & 1046 & 44 & & \\
\hline $\begin{array}{l}\text { SA-PISL } 801 \text { (PI-TOI }-1) \text { ) } \\
\text { SA HDR LO PRESS ALARH }\end{array}$ & $3|i|_{a 1}$ & 1047 & 100 & $90,100 \mathrm{P}$ & \\
\hline $\begin{array}{l}\text { SA-TI-805 (TI TOO A } 5 \text { ) } \\
\text { SERVICE AIR HDR TEMP }\end{array}$ & $3 / 17 \int_{17}$ & Yo47. & 69 & & \\
\hline $\begin{array}{l}\text { DUTY CYCLE } \\
\text { (LOAD IIME/TOTAL T IME) }\end{array}$ & $3 / 9 / 99$ & 1044 & $29 \mathrm{sec} / 7$ & & in \\
\hline RELATIVE HUMIDITY * * & $8 / \sqrt{13} / 52$ & & $n / A$ & & $D / n$ \\
\hline ATMOSPHERIC PRES SURE : & $3 / \operatorname{lol} 97$ & & $N / A$ & & Dn \\
\hline INSIDE TIFMP (IN T01-A) & $3 / 13 / 6$ & & $\pi / A$ & & $D_{4}$ \\
\hline $\begin{array}{l}\text { OUTS SOE TEMPERATURE } \\
\text { (OUTSIDE } 701 \mathrm{~A} \text { ) }\end{array}$ & $3 / 17 / 97$ & & & & $y_{1}$ \\
\hline
\end{tabular}

* Call weather station for required information at 373-2875

Note: If readings are outside designated ranges, Notify Test Director.

(1) See 1030 . T) ATA.

(2) See $\log$ Brok Ratry 3/16/97-1960 wes.

(3) Out of speer. per log sheet, Not Oat of Spees, per OTP. 
HNF-SD-WM-OTR-231, Rev 0

Page 116 of 165

\section{WORKING COPY}

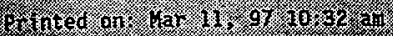

48-HOUR ON-LINE TEST DATA SHEET (Cont.)

OR AIR DRYER/COOLING SKID

\begin{tabular}{|c|c|c|c|c|c|}
\hline PARAMETER & $\mathrm{BATL}$ & TIME & REAOING & EXPECTED RANG & IN1 \\
\hline GLY PUMP RUNING ? & $3 / 12 / 17$ & 1047 & 801 & 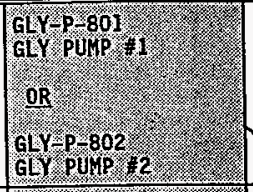 & 4 \\
\hline 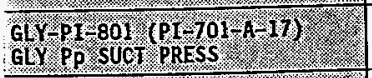 & $3 / 12 / 47$ & 1047 & 3 & 0.10.PSI & \\
\hline 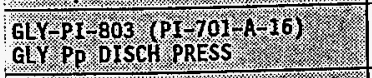 & $3 / 17 / 47$ & $\therefore 1047$ & & 65.75 PSI & \\
\hline G(YT) & $3 / 17 / 97$ & 1048 & & 1001120 & \\
\hline 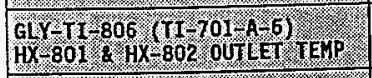 & $3 / 17 / 47$ & 1048 & 79 & $100 \div 120$ & \\
\hline 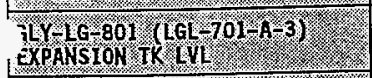 & $3 / 17 / 67$ & 7048 & & $1 / 3=2 / 3, F$ & \\
\hline $\begin{array}{l}\text { IA }=\text { PI } 819 \\
\text { IA THR } 801 \text { PRESS }\end{array}$ & $3 / 17 / 41$ & 1049 & 105 & $80-105 \mathrm{PSI}$ & \\
\hline $\begin{array}{l}\text { MA PI } 820 \text {. } \\
\text { IA PURGE PRSS }\end{array}$ & $3 / 17 / 57$ & 1049 & $107 R_{0}(1)$ & $40 \div 60.951$ & 4 \\
\hline $\begin{array}{l}\text { IAPI } 818 \text {. } \\
\text { IA THR } 802 \text { PRESS }\end{array}$ & $3 / 12 / 47$ & 1049 & 104 & $80-105.95$ & \\
\hline 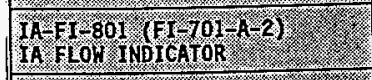 & $3 / 17 / 67$ & 1049 & $20 @$ & $45=90.65$ & \\
\hline 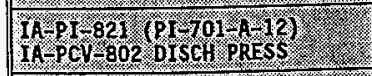 & $3 / 67 / 47$ & 1049 & 42 & $30=45 \times 51$ & \\
\hline 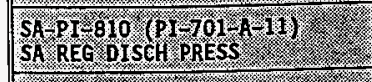 & $3 / 17 / 47$ & 1049 & 40 & $25-45$ & $h$ \\
\hline $\begin{array}{l}\text { BLOW DOWN PREMUTER, } \\
\text { AFTERFILIER. RECEIYER TINRS }\end{array}$ & $3 / 12 / 91$ & & & & $D_{h}$ \\
\hline (1) & & & & & \\
\hline $\begin{array}{l}\text { (1) System Between } C_{4} \\
\text { (2) Low Air usage. }\end{array}$ & s. & & & & $\because$ \\
\hline
\end{tabular}


HNF-SD-WM-OTK-231, ReV 0

Page 117 of 165

48-HOUR ON-!INE TEST DATA SHEET (Cont.)

FOR SA-CMP-802

\begin{tabular}{|c|c|c|c|c|c|}
\hline ARAMETER & $\mathrm{DATE}$ & IIIIE & READING & EXPECTED RANGE & $\mathrm{INI}_{2}$ \\
\hline $\begin{array}{l}\text { 4. } \mathrm{PDI} 802(\mathrm{DPI} 701 / \mathrm{AO2}) \\
\mathrm{PP} 802 \text { SUCT FLT }\end{array}$ & $3 / 17 / 97$ & 1100 & 2 & 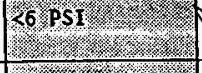 & 1) \\
\hline $\begin{array}{l}\text { APPI } 802 \text { (PI 7O1 A } 6) \\
\text { EMOTE BRG OILPRESS }\end{array}$ & $3 / 17 / a 7$ & 1100 & 38 & $15-160.65$ & \\
\hline $\begin{array}{l}\text { A-PI } 806 \text { (PI } 701-A=5) \\
\text { NTER STG DISCH PRES }\end{array}$ & $3 / 17 / 97$ & 1101. & 29 & $20-40 \%$ & \\
\hline $\begin{array}{l}\text { LYFG } 806 \text { (SF } 701 / A \text { A) } \\
A-C H P=802 ~ H P \text { SIDE GLY }\end{array}$ & $3 / 1$ & 1100 & & Fon & \\
\hline $\begin{array}{l}\text { A-PI-804 (PI-701-A } 8) \\
\text { MP } 802 \text { DISCH PRESS }\end{array}$ & & 1102 & 110 & $85-105$ & \\
\hline $\begin{array}{l}\text { A-TI-802 (TI-701-A } 4) \\
\text { MP } 802 \text { SA DISCH TIEM }\end{array}$ & $3 / 17$ & 1101 & & $90-120$ & \\
\hline $\begin{array}{l}\text { A-SG } 802 \\
\text { A CHP OIL LVL }\end{array}$ & 31 & 1100 & & ABOVE & \\
\hline $\begin{array}{l}\text { iLY-TI-804 (TI-701-A } 10) \\
\text { iA-CHP-802 GLY INLE TEP }\end{array}$ & $8 / 17 / 47$ & 1103 & 27 & $90-120$ & \\
\hline $\begin{array}{l}\text { iLY TI-802 (TI-701-A } 12) \\
\text { iA-CHP } 802 \text { GLY DISCH TEMP }\end{array}$ & $4 / 17 / 97$ & 1103 & 18 & $90-120$ & \\
\hline 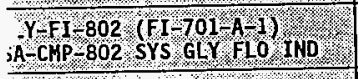 & 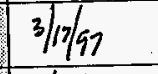 & 1103 & 49 & K67.GPH & $\underline{L}$ \\
\hline $\begin{array}{l}\text { SA PISL }-801 \text { (PI-701 A } 1) \\
\text { SA HDR LO PRESS ALARH }\end{array}$ & $3 / 17 / 45$ & 1103 & & 90.100 & \\
\hline 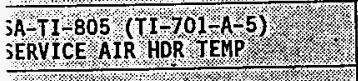 & $\sqrt[3]{17} / 97$ & 1600 & & $90-120$ & \\
\hline $\begin{array}{l}\text { WTY CYCLE } \\
\text { LOAD FIME/TOTAL TIME) }\end{array}$ & $3 / 19 / 97$ & & $\begin{array}{l}2 \pi \\
29 x\end{array}$ & & \\
\hline RELAT IVE HUMIDITY , & $3 / 18 / 12$ & & & & \\
\hline ATMOSPHERIC PRESSURE & & & & & \\
\hline INSIDE TEMP ( $(\mathrm{N} / \mathrm{O}) \mathrm{N}$ ) & $3 / 17 / 67$ & & & & \\
\hline $\begin{array}{l}\text { OUTSIDE TEMPERATURE } \\
\text { (OUTSIDE TOLA) }\end{array}$ & $\sqrt[3]{m} / 97$ & & & & D \\
\hline
\end{tabular}

* Call weather station for required information at 373-2875

Note: If readings are outside designated ranges, Notify Test Director.

(1) Sec 1030 DATA

(2) see log Book Futry $3 / 10 / 17$ - rato Hks.

(3) out of spees. Per $\log$ slact Not out of spece, per OTP 

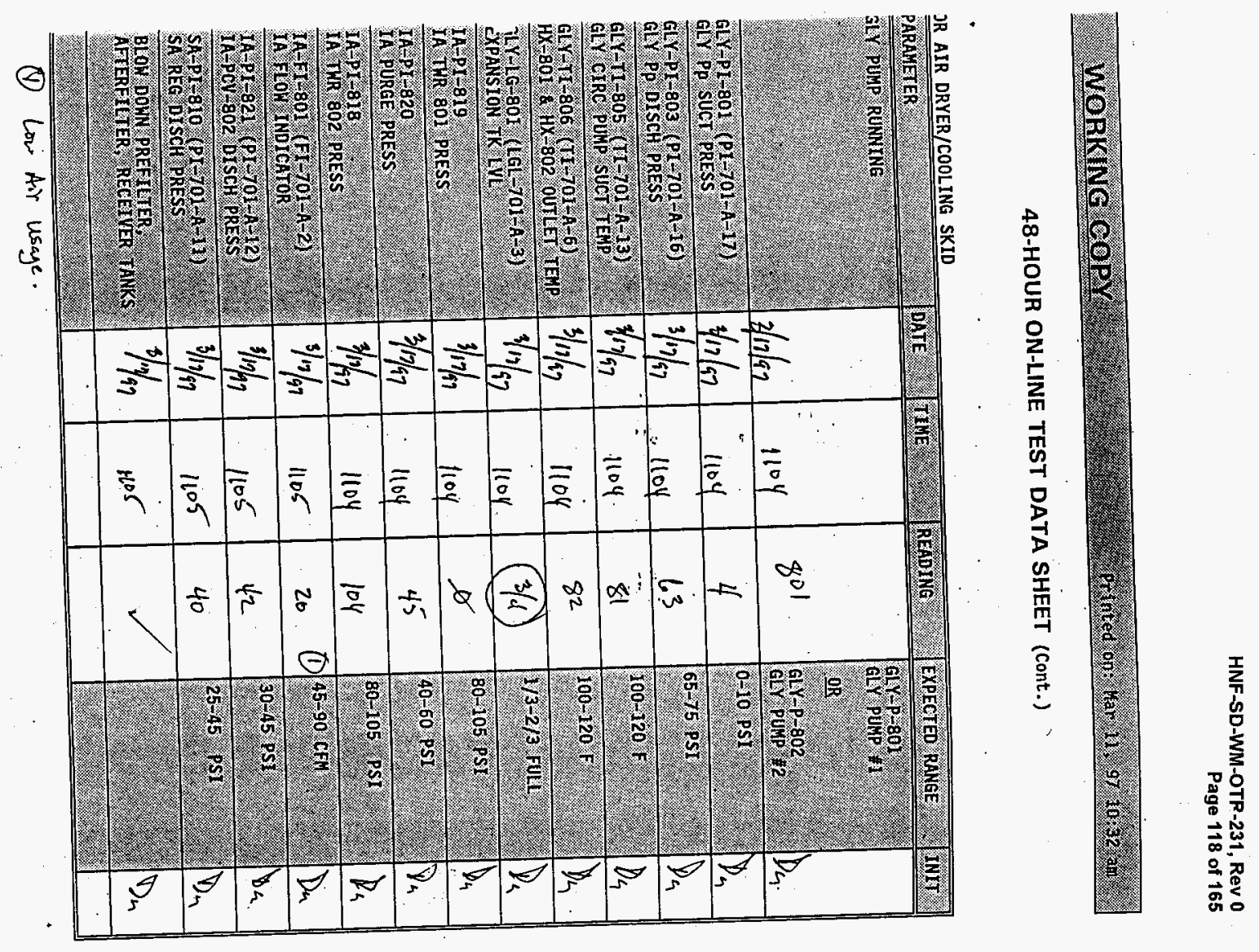
HNF-SD-WM-OTR-231, Rev 0

Page 119 of 165

48-HOUR ON-I!NE TEST DATA SHEET ?:ant:

FOR SA-CMP-BO2

\begin{tabular}{|c|c|c|c|c|c|}
\hline ARAMETER & DATE & $\mathrm{IME}$ & READING & EXPECTED RANGE & INH \\
\hline $\begin{array}{l}\text { A PDI-802 (OPI } 701 \text { AO2) } \\
\text { MP } 802 \text { SUCT FLT D P }\end{array}$ & of $\left.17\right|_{47}$ & 1115 & 2.0 & $6 \times \mathrm{PSI}$ & \\
\hline 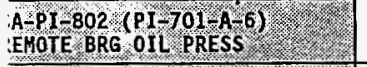 & $3 /\left.19\right|_{41}$ & 1115 & 35 & $15 \% 69.69$ & \\
\hline $\begin{array}{l}\text { NAPT } 806 \text { ( }(\mathrm{PI}-701-\mathrm{h} 5) \\
\text { NTER STG DISCH PRESS }\end{array}$ & $3 / 12 / 97$ & 1115 & 29 & $20-40$ p & \\
\hline 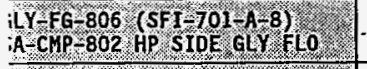 & $3 / 17 / 97$ & ney & & F O O & \\
\hline $\begin{array}{l}\text { A PI- } 804 \text { (PI-701 A } 8) \\
\text { MP } 802 \text { DISCH PRESS }\end{array}$ & $3 / 17 / 97$ & $\operatorname{lll}_{t 15}$ & 110 & $85-105 ?$ & 4 \\
\hline $\begin{array}{l}\text { AA-TI-802 (TI-701 A A } 4 \text { ) } \\
\text { :MP } 802 \text { SA DISCH TEMP }\end{array}$ & $3 / 17$ & 4165 & r & $90-120$ & \\
\hline $\begin{array}{l}3 A-S G-802 \\
3 A \text { CHP } 0 I \text { LVL }\end{array}$ & $3 / 17 / 47$ & $\{114$ & & ABOVE MA & \\
\hline $\begin{array}{l}3 \mathrm{HY}-\mathrm{TI}-804 \text { (TL } 70 \mathrm{~A}-10) \\
3 \mathrm{~A}-\mathrm{CHP}-\mathrm{BO} 2 \mathrm{GLY} \text { INLT TEMP. }\end{array}$ & $3 / 17 / 1$ & 1114 & 81 & $90,120.9$ & \\
\hline $\begin{array}{l}3 L Y-T I-802,(T I-701-A-12) \\
3 A-C H P-802 \text { GLY DISCH IEMP) }\end{array}$ & $3 / 17 / 8$ & 1116 & 82 & $90-120 \%$ & \\
\hline 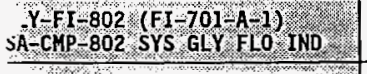 & $3 / 19 / 97$ & 1116 & 49 & $667 \mathrm{GPH}$ & \\
\hline $\begin{array}{l}\text { SA PISL 801 (PI-701-A } 1 \text { ) } \\
\text { SA HDR LO PRESS ALARA }\end{array}$ & $3 / 17 / 11$ & 1112 & $10^{\circ}$ & 90.100 PSI & \\
\hline $\begin{array}{l}\text { SA-TI-8OS (TI-701-A } 5 \text { ) } \\
\text { SERVICE AIR HDR TIEPP }\end{array}$ & $8 / 46 / 47$ & 1117 & 78 & $90-120 \%$ & \\
\hline $\begin{array}{l}\text { DUTY CYCLE } \\
\text { (LOAD TIME/TOTAL, TIME) }\end{array}$ & $3 / 10 / 41$ & 1115 & $46 / 54$ & & \\
\hline RELAIIVE HUMIDITY 7 & $3 / 19 / 17$ & & (1) & & \\
\hline ATMOSPHERIC PRESSURE & $2 / 13 / 42$ & & & 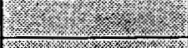 & \\
\hline INSIDE TEMP. $\left(\mathrm{IN} \mathrm{r}_{01} \mathrm{~A}\right)$ & $3 / 1 \% / 62$ & & & & \\
\hline $\begin{array}{l}\text { OUTSIDE TEMPERATUR? } \\
\text { (OUTSIDE } 701-1 \text { ) }\end{array}$ & $3 / 10 / 21$ & & & & \\
\hline
\end{tabular}

* Call weather station for required information at 373-2875

Note: If readings are outside designated ranges, Notify Test Director.

(1) See $1030^{\circ}$ DATt

(E) See log Rode Entry $3 / 16 / 97$ - 1940 Hes

(3) Dot of spen, Po log sheets, Not Out of speer, per OTP. 
48-HOUR ON-LINE TEST DATA SHEET (Cont.)

FOR AIR DRYER/COOLING SKID

\begin{tabular}{|c|c|c|c|c|c|}
\hline PARAMETER & DAIE & IIIE & REAGING & EXPECTEO RANG & $1 N 1$ \\
\hline 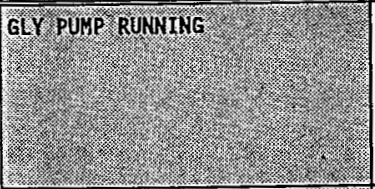 & $3 / 12 / 12$ & 1117 & 801 & 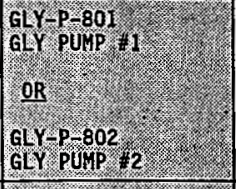 & \\
\hline 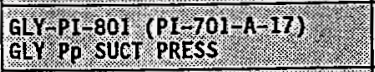 & $8 / x / 47$ & 1118 & 4.5 & 0.10. PSI & \\
\hline 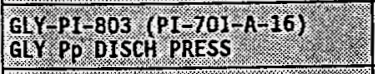 & $7 / 2 / 14$ & 1118 & $k 4$ & $65-75$ PSI & \\
\hline 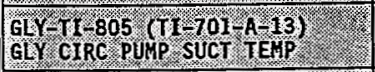 & $1 / 9 / 19$ & 1118 & 84 & $100-120 \%$ & \\
\hline 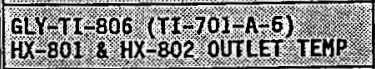 & $3 / 27 / 11$ & 1118 & $<0$ & $100-120$ & $D_{4}$ \\
\hline 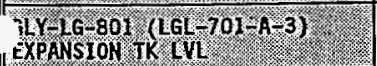 & $3 / 67 / 94$ & 1118 & & $1 / 3=2 / 3 \cdot 4$ & \\
\hline $\begin{array}{l}\text { IA PI } 819 \text {. } \\
\text { IA THR } 801 \text { PRESS }\end{array}$ & $3 / 1 / 2 / 81$ & 9119 & 104 & $80-105.95$ & \\
\hline $\begin{array}{l}\text { 1H PI R2O } \\
\text { TA PURG PRESS }\end{array}$ & $3 / 17 / 19$ & 1119 & $104(2)$ & $40-60.951$ & \\
\hline 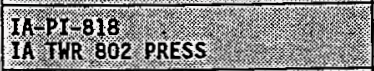 & $2 / 2 / 91$ & 1119 & 104 & $80-105 \%$ ps & \\
\hline 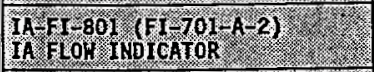 & $\sqrt[3]{17}$ & 1119 & 20 & $45-90.6$ & \\
\hline 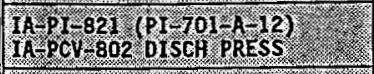 & $3 / 2 / 19$ & 1119 & 42 & $30=45.951$ & \\
\hline 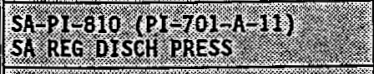 & $y / 1 / 4$ & 1119 & 40 & $25=45$ ? & \\
\hline 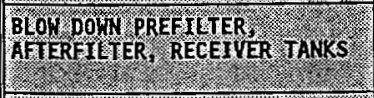 & $9 / 1 / 4$ & 1119 & & & \\
\hline 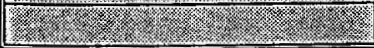 & & & & 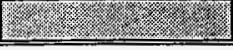 & \\
\hline
\end{tabular}

(1) Low Ait Usage.

(2) Hir Dryer Betwrem Regeneration Cyales.

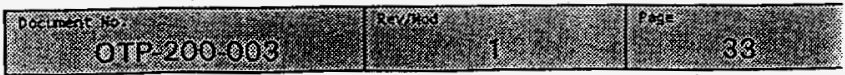


HNF-SD-WM-OTR-231, Rev 0

Page 121 of 165

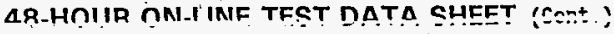

FOR SA-CMP-802

\begin{tabular}{|c|c|c|c|c|c|}
\hline ARAMETER & DATE & TIME & READIHG & EXPECTED RAMGE & INIT \\
\hline $\begin{array}{l}\mathrm{APPI} 802 \text { (OPI-701 AO2) } \\
\mathrm{MP} 802 \text { SUCT FLT D } / \mathrm{P}\end{array}$ & $3 / n k ?$ & 11130 & 2.0 & 6. psI. & \\
\hline $\begin{array}{l}\text { ¿API-802 (PI 701-A } 6) \\
\text { LEMOTE BRG OIL PRESS }\end{array}$ & $3 / 12 / 97$ & 1129 & 3 & $15 \% 0$ & \\
\hline $\begin{array}{l}\text { SA-PI }-806 \text { (PI J01-A S) } \\
\text { INTER STG DISCH PRESS }\end{array}$ & $3 / 12 / 49$ & 1131 & 8 & $20=40$ & 24 \\
\hline 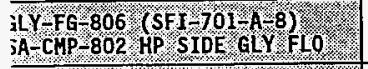 & $3 / 17$ & 1129 & & HLOH\% & \\
\hline $\begin{array}{l}\text { \$A PI } 804\left(\mathrm{PI}-701-{ }^{-}-8\right) \\
\text { MPP } 802 \text { DISCH PRESS }\end{array}$ & & 1132 & 1103 & $85-105$ & \\
\hline $\begin{array}{l}\text { SA-TI-802 (TI-701-A-4) } \\
\text { MPP } 802 \text { SA DISCH IEMP }\end{array}$ & $3 / 17$ & 1131 & 110 & & \\
\hline $\begin{array}{l}\text { SA-SG } 802 \\
\text { SA CMP } 0 I L \text { LV }\end{array}$ & & 1129 & & ABOYE & \\
\hline $\begin{array}{l}\text { GLY TI-804 (TI-701 A A I0) } \\
\text { SA-CHP } 802 \text { GLY INLET TEMP. }\end{array}$ & $3 / 12 / 97$ & 1132 & 84 & & \\
\hline $\begin{array}{l}\text { GLY-TI-802 (TI-701-A } 12) \\
\text { SA-CMP- } 802 \text { GLY DISCH TEMP. }\end{array}$ & & 1132 & 85 & $90-120$ & \\
\hline $\begin{array}{l}\mathrm{Y}-\mathrm{FI} 802 \text { (FI-701 } \mathrm{A}-1 \text { ) } \\
\mathrm{SA}-\mathrm{CMP}-802 \text { SYS GLY FLO IND }\end{array}$ & $3 / 17 / 91$ & 1132 & 19 & $667, \mathrm{GPH}$ & \\
\hline $\begin{array}{l}\text { SA-PISL- } 801 \text { (PI-701-A-1) } \\
\text { SA HDR LO PRESS ALARH }\end{array}$ & inglan & 1133 & 100 & $90 \div 100 \% \mathrm{pSI}$ & \\
\hline $\begin{array}{l}\text { SA-TI-805 (TI-701-A } 5 \text { ) } \\
\text { SERVICE AIR HDR TEMP }\end{array}$ & & $1|3|$ & 82 & & \\
\hline $\begin{array}{l}\text { DUTY CYCLE } \\
\text { (LOAD TIME/TOTAL TIME) }\end{array}$ & $\mid x / n / \infty 1$ & $|13|$ & $298 \times$ & & \\
\hline RELATIVE GUMIDITY $t$, & $3 / m / a n$ & & & & \\
\hline ATMOSPHERIC PRESSURE & $3 \sqrt[3]{1 / 61}$ & & & & 4 \\
\hline INSIDE TENP (N TO1 $-\mathrm{A})$ & $9 \sqrt[19]{51}$ & & & & $1)_{4}$ \\
\hline $\begin{array}{l}\text { OUTSIDE TEMPERATURE } \\
\text { (OUTSIDE TOI-A) }\end{array}$ & $2 / 14 / 01$ & & & & $D$ \\
\hline
\end{tabular}

* Call weather station for required information at 373-2875

Note: If readings are outside designated ranges, Notify Test Director.

(1) Sec 1030 DATAt

(2) see $\log$ Brode Eutry 3/10/17-1940 Hes.

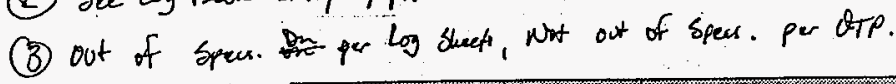




\section{8-HOUR ON-LINE TEST DATA SHEET (cont.)}

FOR AIR DRYER/COOLING SKID

\begin{tabular}{|c|c|c|c|c|c|}
\hline PARAMETER & DATE & MHE & READING & EXPECTED RANGE & 111 \\
\hline 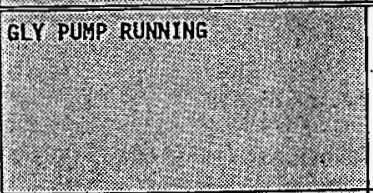 & $z_{(2) / 47}$ & 1133 & 80 & 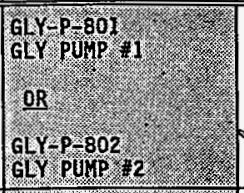 & \\
\hline 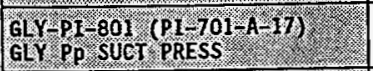 & $3 / 19$ & 1153 & 5 & 0.10.psI & \\
\hline 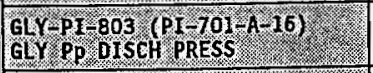 & $2 / n / a 1$ & 1133 & & $65-75 \% \mathrm{sI}$ & \\
\hline 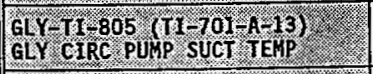 & $3 n$ & 1133 & 86 & $100-120.7$ & \\
\hline 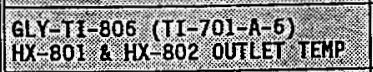 & $1 / 9$ & 1134 & & $100-120 \%$ & \\
\hline 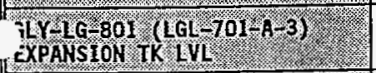 & $\sqrt[3]{19} / 41$ & 1134 & & $13-2 / 3$ & \\
\hline $\begin{array}{l}\text { IA-PI. BIg } \\
\text { IA WR 801 PRESS }\end{array}$ & $\sqrt[3]{121}$ & 1134 & & 80 & \\
\hline WAPT KRO & 1741 & 1134 & & $40=60 . \mathrm{PSI}$ & \\
\hline IA PI 1818 , PRES & $3 / 19 / x$ & 1134 & & $80-105 \%$ pSI & 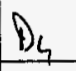 \\
\hline 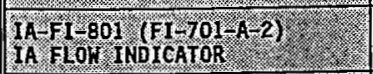 & $5 / 14 / 91$ & N35 & (1) & 45-90\% & Dh \\
\hline 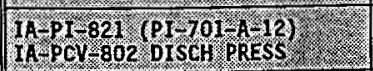 & $3 / 19 / 91$ & 1135 & 1 & $30-45.951$ & $D_{4}$ \\
\hline 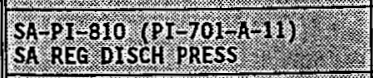 & $5 / m / n$ & 1135 & 40 & 25.45 .051 & $D_{h}$ \\
\hline BLON DOWN PRE TITER & $3 / 12 / 9 n$ & 1635 & & & \\
\hline (2) & & & & 7 & \\
\hline
\end{tabular}

(1) Low Air Usage. 
HNF-SD-WM-OTR-231, Rev 0 Page 123 of 165

AR-HNIIR OM!-IM!E TEST DATA SHEET !ront !

FOR SA-CMP-802

\begin{tabular}{|c|c|c|c|c|c|}
\hline DARAMETER & DATE \% & TIME & READWG & EXPECTED RANGE & $\mathrm{MIT}$ \\
\hline $\begin{array}{l}\triangle A-P D I-802,(O P I-701-A O 2) \\
\text { CHP } 802 \text { SUCT } F L T \text { D } / P\end{array}$ & $3 / 17 / 97$ & 1229 & & 6. PST. & 10 \\
\hline $\begin{array}{l}\text { SA-PI 8O2 (PI- J01 A } 6) \\
\text { REMOTE BRG OIL PRESS. }\end{array}$ & $3 / 12 / 9$ & 1229 & & $15=665$ & \\
\hline $\begin{array}{l}\text { SA-PI } 806 \text { (PI-101) A } 5 \text { ) } \\
\text { INTER STG DISCH PRESS }\end{array}$ & $3 / 7$ & 1229 & & $20=40.85$ & \\
\hline $\begin{array}{l}\text { GLY FG } 806 \text { (SFI-701-A 8) } \\
\text { SA-CKP } 802 \text { HP SIDE GLY LLO }\end{array}$ & $3 / 19 / 49$ & 1229 & & HLor, & \\
\hline $\begin{array}{l}\text { SA PI }-804 \text { (PI } 701 / \mathrm{A}=8) \\
\mathrm{CHP} 802 \text { DISCH PRESS }\end{array}$ & $\sqrt[3]{17 / 41}$ & 1229 & & $85 \div 105$ & \\
\hline $\begin{array}{l}\text { SA-T I- } 802 \text { ( (TI } 701-A-4) \\
\text { CMP } 802 \text { SA DISCH TEMP) }\end{array}$ & 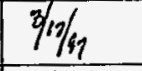 & 1229 & & $90-120$ & \\
\hline $\begin{array}{l}\mathrm{SA}-\mathrm{SG}-802 \\
\mathrm{SA} \text { CHP } 0 \mathrm{IL}, \mathrm{LVL}\end{array}$ & $3 / 17 / 91$ & 1229 & & ABOVE & \\
\hline $\begin{array}{l}\text { GLY TII- } 804 \text { (TI-701-A-10) } \\
\text { SA-CHP } 802 \text { GLY TNLET TTEP }\end{array}$ & $3 / 17 / 91$ & 1229 & & $90-120$ & \\
\hline $\begin{array}{l}\text { GLY }-\mathrm{T}-802 \text { (TI } 701-A-12) \text {, } \\
\mathrm{SA}-\mathrm{CHP}-802 \text { GL DISCH TEMP }\end{array}$ & $3 / 17 / 11$ & 1229 & & $90-120 \%$ & \\
\hline $\begin{array}{l}\mathrm{YAF}-802 \text { (EI } 701-\mathrm{A}-1) \\
\mathrm{A}-\mathrm{CH}-802 \text { SYS GLY }\end{array}$ & & $2 \quad 2.9$ & & $267 . \mathrm{GPH}$ & \\
\hline $\begin{array}{l}\text { SA } \\
\text { SA HDR L L O PRESS ALARH }\end{array}$ & $3 / 19 / 9$ & 12 & & $90=100 \% \mathrm{PS}$ & \\
\hline $\begin{array}{l}\text { SA-II-805 (TI-7O1 A-5) } \\
\text { SERVICE AIR HDR TEPP) }\end{array}$ & $7 / 1 / 11$ & 1229 & & $90-120 \mathrm{~F}$ & \\
\hline $\begin{array}{l}\text { DUTY CYCLE } \\
\text { (LOAD TIMEITOTAL T THE) }\end{array}$ & $3 / 14$ & 123 & & & \\
\hline RELATIVE HUMIDITY & $3 \sqrt[1079]{41}$ & 1230 & (1) & & \\
\hline ATMOSPHERIC PRESSURE / + / & $3 / 17 / 91$ & 1230 & (1) & & \\
\hline INSIDE IEFP ( $(\mathrm{N} / 0 \mathrm{O}-\mathrm{A})$ & 9196 & 1230 & 23 & & \\
\hline $\begin{array}{l}\text { OUTSIDE TEMPERATURE } \\
\text { (OUTSIDE } 101 \% \text { A) }\end{array}$ & ithet & 17 & & & 10 \\
\hline
\end{tabular}

* Call weather station for required information at 373-2875

Note: If readings are outside designated ranges, Notify Test Director.

(1) See Darit From 1030 Reabings.

(2) See $\log$ Book Enty 3/10/97-1940 Hes.

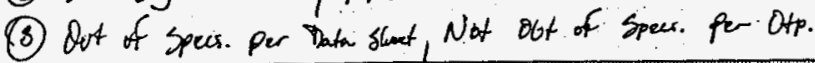


48-HOUR ON-LINE TEST DATA SHEET (Cont.)

\begin{tabular}{|c|c|c|c|c|c|}
\hline 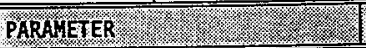 & DATE & TIUE & READING & EXPET EV RA & III \\
\hline GLP PUMP RUMALS & $3 / 14 / 40$ & & & 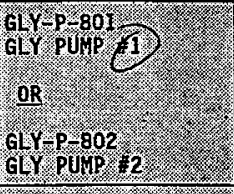 & \\
\hline 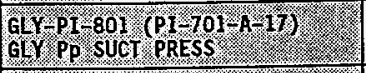 & $2 / 49$ & 1233 & 6 & O. $10.9 S T$ & \\
\hline 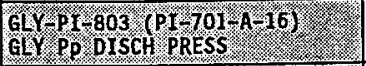 & $3 / m / a n$ & 12.33 & 60 & $65-75$ PSI & \\
\hline 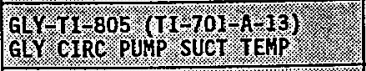 & $1 / 1919$ & 1233 & & $100-120.6$ & A \\
\hline 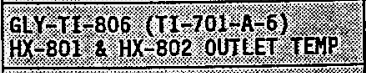 & $3 / 1 / 97$ & $123 y$ & & $100-120,=$ & \\
\hline 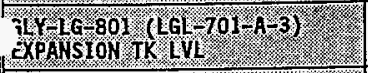 & $3 / 7 / 99$ & 12 & & $13=2 / 3.60$ & \\
\hline 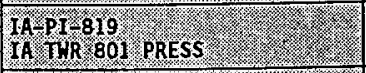 & $3 / 4 / 91$ & 12 & & 80.05 .051 & $\Phi$ \\
\hline $\begin{array}{l}\text { MAPI } 820 \% \\
\text { MA PUREE PRESS. }\end{array}$ & $3 / 19 / 57$ & 1234 & & $40=60$ PSI & \\
\hline MA PI-818 & $3 / 1 / 11$ & 1235 & & $80-105-P S I$ & \\
\hline 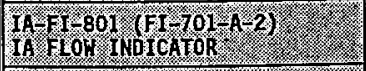 & $7 / 7 / 49$ & 12 & 20 & $45-90.654$ & $\sqrt{18}$ \\
\hline 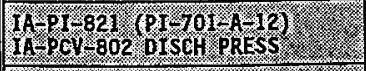 & $3 / 19 / 40$ & 12 & & $30-45 \%$ & 8 \\
\hline 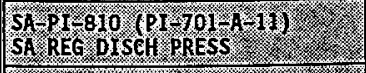 & $3 / 19 / 9$ & 1236 & & $25-45.951$. & \\
\hline 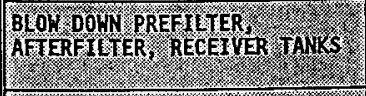 & $1 / 19 / 19$ & 36 & & & \\
\hline |l, & & & & & \\
\hline
\end{tabular}

(1) Low Air Usagie. 
HNF-SD-WM-OTR-231, Rev 0

Page 125 of 165

\section{8-HOUR ON-LINE TEST DATA SHFET ([nnt.)}

FOR SA-CMP-802

\begin{tabular}{|c|c|c|c|c|c|}
\hline DARAMETER & DATE & TLE & READING & EXPECTED & 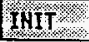 \\
\hline 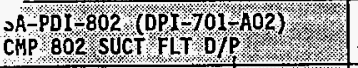 & $3 / 1797$ & 1330 & 2.0 & 6.pSI & \\
\hline $\begin{array}{l}\text { SA-PI } 802 \text { ( } P I-701 / \mathrm{A} 6) \\
\text { REMOTE VBRG OILPRESS }\end{array}$ & $3 / 12 / 97$ & 1330 & 28 & $15-4 \% \times 65$ & \\
\hline $\begin{array}{l}\text { SA PI } 806 \text { (PI 7OI A } 5 \text { ) } \\
\text { INTER STG DISCH PRESS }\end{array}$ & $3 / 17 / 97$ & 1331 & 28 & $20-40.9$ & \\
\hline $\begin{array}{l}\text { GLY FG-806 }(S F I-701-A-8) \\
\text { SA-CMP } 802 \text { HP SIDE G }\end{array}$ & & 1332 & & FLWF & \\
\hline $\begin{array}{l}\text { SAPI } 804(P 1-701-A-8) \\
\text { CMP } 802 \text { DISCH PRESS }\end{array}$ & $3 / 1$ & 1332 & (3) & $85-105: \mathrm{P}$ & \\
\hline $\begin{array}{l}\text { SA-TI } 802 \text { (TI-7O1 A-4) } \\
\text { CMP } 802 \text { SA DISCH TEMP) }\end{array}$ & $3 / 17 / 97$ & 1331 & (2) & $90-120 \mathrm{~F}$ & \\
\hline $\begin{array}{l}\mathrm{SA}-\mathrm{SG}-802 \\
\mathrm{SA} \mathrm{CHP} O \mathrm{OIL} \mathrm{LVL}\end{array}$ & & 1333 & & ABOVE MAF & \\
\hline 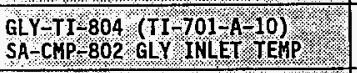 & & 1333 & & $90-120$ & \\
\hline $\begin{array}{l}\text { GLY } 1 \text { I } 802 \text { (TIV701 A } 12) \\
\text { SA-CHP } 802 \text { GLY DISCH TEMP }\end{array}$ & & 1333 & $t_{t \rightarrow 0}^{101}$ & $90-120 \%$ & \\
\hline 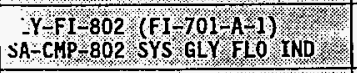 & & 1333 & 49 & K67 GPH & \\
\hline $\begin{array}{l}\left.\text { SA-PISL } 801 \text { (PI } 701-1)^{-1}\right) \\
\text { SA HDR LO PRESS ALARI }\end{array}$ & & 1334 & $10^{0}$ & $90 \% 100.8$ & \\
\hline $\begin{array}{l}\text { SA-TI-805 (TI-701/A-5) } \\
\text { SERVIGE AIR HDR TEMP }\end{array}$ & $3 / 17 / 97$ & 1330 & 96 & $90=120 \%$ & \\
\hline $\begin{array}{l}\text { DUTY cYGL } \\
(\mathrm{LOAD} \text { TIME } / \mathrm{TOTAL} \text {, IME) }\end{array}$ & & 1332 & 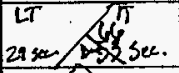 & & \\
\hline RELAIIVE HUMIDHTY & & & 71) & 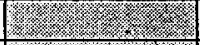 & \\
\hline ATMOSPHERIC PRESSURE & $3 \sqrt[37]{197}$ & & (i) & & \\
\hline INSIDE TEMP $(1 \mathrm{~N} / 01-\mathrm{A})$ & $2 / 179$ & 1324 & 73 & 1 . & \\
\hline $\begin{array}{l}\text { OUTSIDE TEMPERATURE } \\
\text { (OUTSIDE } 701 \text { A) }\end{array}$ & $3 / 1 / 47$ & 1326 & 63 & & \\
\hline
\end{tabular}

* Call weather station for required information at 373-2875

Note: If readings are outside designated ranges, Notify Test Director.

(1) See $1030^{\circ}$ DAtA.

(2) See log Book Zendry 2/10/97-1940 LfRs

(3) Qut of Speer. per log shect, Not Dut of speer. Per Orp 


\section{WORKING COPY}

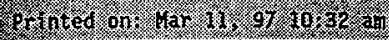

48-HOUR ON-LINE TEST DATA SHEET (Cont.)

FOR AIR DRYER/COOLING SKID

\begin{tabular}{|c|c|c|c|c|c|}
\hline PARAMEIER & DATE & IIME & REAOIIG & EXPECTED RANG & $1 \mathrm{NI}:$ \\
\hline GLY RUMP RUMNING & $3 / 12 / 97$ & 1334 & $8 \alpha$ & 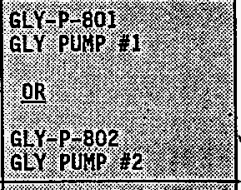 & Dan \\
\hline 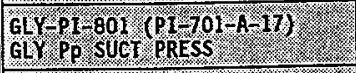 & $2 / 1769$ & 1335 & $\eta .5$ & 0.10.psI & \\
\hline 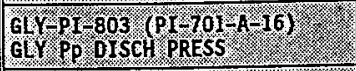 & $5 / 17 / 47$ & 1335 & & $65.75 \cdot 951$ & \\
\hline 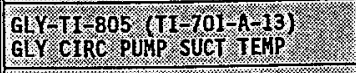 & $8 / 17 / 97$ & 1335 & 102 & $100-120 \%$ & h \\
\hline 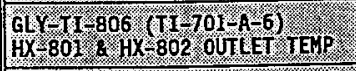 & & 1334 & $1 / 03$ & $100120 \%$ & \\
\hline 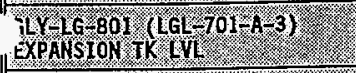 & $8 / 1$ & 1336. & & $1 / 3-2 / 3.1$ & \\
\hline 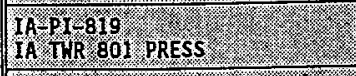 & $\sqrt[3]{17 / 17}$ & 1334 & & 80 105 PST & \\
\hline $\begin{array}{l}\text { IA P P } 820 \text {, } \\
\text { MA PURGE PRESS }\end{array}$ & $3 / 1$ & 1336 & & 40-60\% 95 & \\
\hline $\begin{array}{l}\text { IA PI gig } \\
\text { IA THR } 802 \text { PRESS }\end{array}$ & $3 /$ & 1336 & 104 & 80-105. PsI & \\
\hline 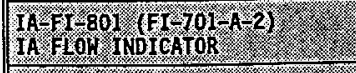 & 3 & 1337 & 20 & 45.90 .96 & \\
\hline 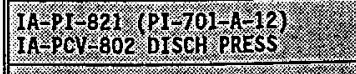 & $3 /$ & 1337 & 42 & $30=45 \times 19$ & \\
\hline 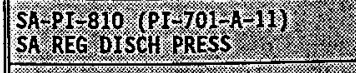 & $3 / 17 / 7$ & 1337 & 40 & 25.45 . PSI & \\
\hline 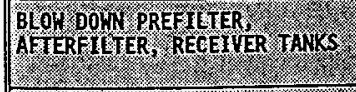 & $8 / m / 91$ & 1337 & & & Li \\
\hline 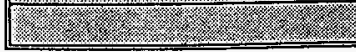 & & & & 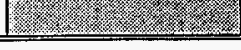 & \\
\hline
\end{tabular}

(1) Low Air Usage

P.


48-HOUR ON-LINE TEST DATA SHEET !r.nnt.)

FOR SA-CMP-802

\begin{tabular}{|c|c|c|c|c|c|}
\hline PARAMETER & DATE, 0.2 & TIHE. & READING & EXPECTED RANGE & INIT \\
\hline 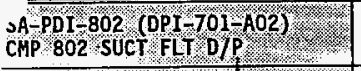 & $3 / 12$ & 1429 & 2.0 & K6 PSI & \\
\hline $\begin{array}{l}\text { SA-PI-802 (PI-701-A } 6) \\
\text { REMOTE BRG OIL PRESS }\end{array}$ & $3 / 17 / 57$ & 1429 & 23 & $15-10.6$ & $v$ \\
\hline $\begin{array}{l}\text { SA-PI-806 (PI } 701 / 1 / 5) \\
\text { INTER STG DISCH PRES }\end{array}$ & $3 / 17$ & 1429 & 29 & 20-40.PS & \\
\hline 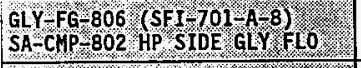 & $3 / 17 / 97$ & 1428 & & $\mathrm{FlON}$ & \\
\hline $\begin{array}{l}\text { SA-PI-804 }(\mathrm{PI}-701-\mathrm{A}-8) \\
\text { CHP } 802 \text { DISCH PRESS }\end{array}$ & & 1430 & 110 & $85.105 \times$ & \\
\hline $\begin{array}{l}\text { SA-TI-802 (TI-701-A-4) } \\
\text { CHP } 802 \text { SA DISCH TEFP }\end{array}$ & $3 / 17 / 97$ & 1429 & $185 \%$ & $90-120 \mathrm{~F}$ & \\
\hline $\begin{array}{l}\text { SA-SG } 802 \\
\text { SA CMP } 0 \mathrm{IL} \text { L VL }\end{array}$ & & 1428 & & ABOVE MA & \\
\hline 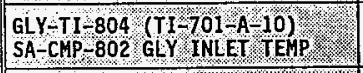 & & 1430 & 10 & $90-120$ & \\
\hline $\begin{array}{l}\text { GLY }-\mathrm{TI}-802 \text { (TI-701-A-12) } \\
S A-C M P-802 \text { GLY DISCH TEMP }\end{array}$ & 3 & 1430 & 108 & $90-120$ & \\
\hline $\begin{array}{l}Y-F I-802 \text { (FI-7O1 } \\
S A-C H P=802 \text { SYS GLY } 1 \text { FLO IND }\end{array}$ & & 1430 & & 67 GP & \\
\hline $\begin{array}{l}\text { SA PISL } 801 \text { (PI- 7OI A I) } \\
\text { SA HDR L0 PRESS ALARH }\end{array}$ & $3 \sqrt[3]{17 / 97}$ & 1428 & 100 & $90 \% 100 \mathrm{PST}$ & \\
\hline $\begin{array}{l}\text { SA-T1-805 (TI-701,A-5) } \\
\text { SERVICE AIR HDR TEMP }\end{array}$ & $3 / 17 / 97$ & 1427 & $m$ & $90 \div 120$ & \\
\hline $\begin{array}{l}\text { DUTY CYCLE } \\
\text { (LOAD TIME /TOTAL TIME) }\end{array}$ & $3 / 17 /$ & 1428. & $\begin{array}{l}2 \pi \\
27 \times 2\end{array}$ & & \\
\hline RELATIVE HUMIDITY $\%$ - & $3 \sqrt[12]{197}$ & & (1) & & \\
\hline ATHOSPHERIC PRESSURE & $3 / 17 / 97$ & & (1) & & \\
\hline INSIDE TEHP (IN 7OIV) & $3 / 17 / 97$ & 1427 & +85 & & Ev \\
\hline $\begin{array}{l}\text { OUTSIDE TEMPERATRE } \\
\text { (OUTSIDE TOI-A) }\end{array}$ & $\sqrt[3]{17 / 97}$ & 1427 & 58 & 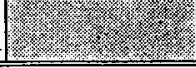 & \\
\hline
\end{tabular}

* Call weather station for required information at $373-2875$

Note: If readings are outside designated ranges, Notify Test Director.

(1) See 1030 DATA

(2) Dee log Book Eutry $\% 1 \% / \pi$ - 1940 HAs.

(3) Qot of speen. per latoun sheet, Not out of Spers. Per OTP. 
HNF-SD-WM-OTR-231, Rev 0

Page 128 of 165

\section{WORKING COPY}

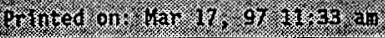

\section{8-HOUR ON-LINE TEST DATA SHEET (Cont.)}

FOR AIR DRYER/COOLING SKID

\begin{tabular}{|c|c|c|c|c|c|}
\hline BARAHEIER & DATE & WHE & REAPING & EXPECTED RANGE & init \\
\hline 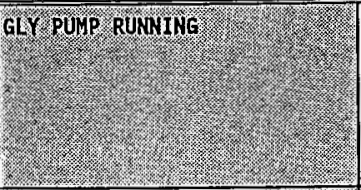 & $3 / 17 / 97$ & 1431 & 801 & 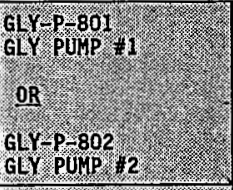 & 4 \\
\hline $\begin{array}{l}\text { GLY P I } 801 \text { ( (PI } 701-A-17) \text {, } \\
\text { GY PP SUC PRESS }\end{array}$ & $3 / 17 / 97$ & 1431 & 9 & 0-10. PSI & bn \\
\hline $\begin{array}{l}\text { GLY PI } 803 \text { (PI 7OI-A-16) } \\
\text { GLY PP DISCH PRESS }\end{array}$ & $\frac{3}{21} 17 / 97$ & 1431 & $\zeta$ & $66-75$ pSI & 4 \\
\hline 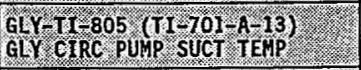 & $3 / 17 / 97$ & 1431 & 110 & $100=120$ & \\
\hline 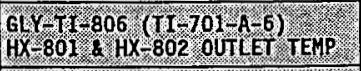 & $3 / 17 / 97$ & 1431 & 110 & $100-120 \cdot \mathrm{F}$ & n \\
\hline 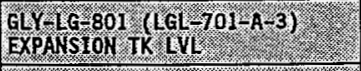 & $3 / 17 / 97$ & 1432 & $3 / 4$ & $1 / 3-2 / 3$ FU & \\
\hline IA P T 819 R 801 PRESS & $3 / 17 / 97$ & 1432 & 105 & $80-105 \cdot p S$ & \\
\hline $\begin{array}{l}\text { MAP } 1820 \\
\text { M P PURGE PRESS }\end{array}$ & $5 / 12 / 47$ & 1432 & 45 & $40=60 \%$ SSI & \\
\hline TA-PI. 818 . & $3 / 17 / 97$ & 1432 & $\varnothing$ & $80 \% 105: ?$ & \\
\hline 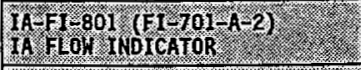 & $z / 12 / 27$ & 1432 & 20 & $45 \div 90.67$ & \\
\hline 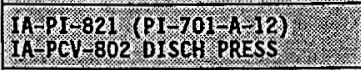 & $3 / 17 / 17$ & 1432 & 42 & $30=75.751$ & n \\
\hline SHPL 10 (PI 70I, U) & $3 / 19 / 17$ & 1432 & 40 & $25 \cdot 45$ & $D_{h}$ \\
\hline 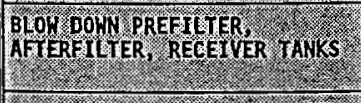 & $3 / 17 / 17$ & 1433 & & & \\
\hline 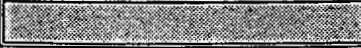 & & & & 20 & \\
\hline
\end{tabular}

(I) Low Ait Usage. 
HNF-SD-WM-OTR-231, Rev 0

Page 129 of 165

\section{WORKING.COPY}

Ho

48-HOUR ON-LINE TEST DATA SHEET (Cont.)

FOR SA-CHP-802

1997

ARAMEIER

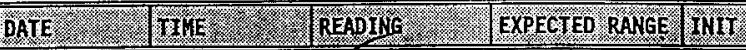

A- DDI-802 (OPI $701 \%$ \%02)

$\mathrm{MP} 802$ SUCT $\mathrm{FL} / \mathrm{O} / \mathrm{P}$

A-P1 2802 - $11-701-\mathrm{A}=6$

EMOTE BRG OL 1 BRSS

A 21 1 806 $(\mathrm{PI} / 701-\mathrm{A} 5)$

NTER STG DISCH PRESS

LY FG 806 (SF -701 / 8 )

A-CHP-802 HP SIDE GLY $\mathrm{FLO}$

A-PI -804 (PI-70I-A-B)

HP 802 DISCH PRESS

A -1 1 802 ( 1 ( 701 A $_{4}$ )

HP 802, SA OISCH TEMP

iA-SG 802

;A CHP. OIL LVL

ifY - I -804 (TI 701 - A 10 )

3A-CHP-802 GLY INLET TENP

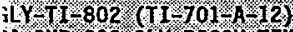

$3 A$ CMP 802 GLY DISCH TEH

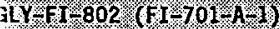

;A-CHP 802 SYS GIY EDO IND

3A PISL 801 (PI-7012. 1 )

SA HDR $\angle O$ PRESS ALARH

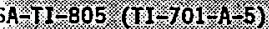

SERYTCE AIR HDR TEHP

JUTY OYCLE

(OOAD IHE LOTAL TIME)

ZELATIVE HUTIDTIY

TIMOSPHERTC PRESSURE

INSIDE TEHP. (IN VIOI)

JUTSTDE TEHPERATURE

(OUTSTEE 701. A)

\begin{tabular}{|c|c|c|c|c|}
\hline $3-17$ & 1529 & $\xi_{1.7}$ & 26 PSI & 16 \\
\hline $3-17$ & 1529 & 22 & & Lo \\
\hline-17 & 1529 & 28 & & 18 \\
\hline 3.17 & 1530 & $V$ & & 10 \\
\hline $3-17$ & 1530 & $106 .(3)$ & & 12 \\
\hline $3-17$ & 1530 & 187 & & $\pi$ \\
\hline $3-17$ & 1530 & ok & & $\pi$ \\
\hline $3-17$ & 1530 & 108 & & $\pi$ \\
\hline $3-17$ & 1530 & 108 & & 40 \\
\hline $3-17$ & 1531 & 50 & & $\pi$ \\
\hline $3-17$ & 1531 & 92 & & $\pi$ \\
\hline $3-17$ & 1531 & 106 & & 7 \\
\hline $3-17$ & 1531 & $2761 \pi$ & & $x$ \\
\hline $3-17$ & 1571 & 0 & & \\
\hline $3-12$ & 153 & 0 & & \\
\hline $3-17$ & 1571 & 26 & f & 42 \\
\hline $3-17$ & 153 & 56 & & 40 \\
\hline
\end{tabular}

* Call weather station for required information at $373-2875$

Note: If readings are outside designated ranges, Notify Test Director.

(1) see is o utite

(O) SeeL.4 BOOK ?/10/97

3) out of secc pert

lat sweet nototp 
HNF-SD-WM-OTR-231, Rev 0

Page 130 of 165

\section{WORKING COPY}

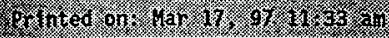

48-HOUR ON-LINNE TEST DATA SHEET (Cont.)

\begin{tabular}{|c|c|c|c|c|c|}
\hline PARAMEIER & DATE & TIME & READING & EXPEGTED RANGE & Wh \\
\hline 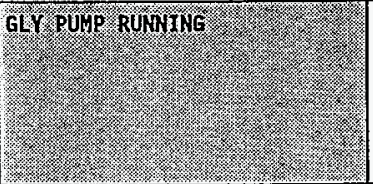 & $3-17$ & & & 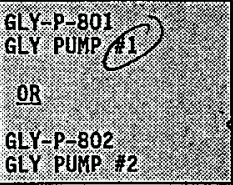 & 16 \\
\hline 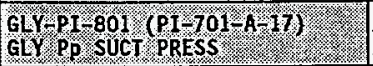 & $3-17$ & 1532 & & 0.10 PSI & 19 \\
\hline $\begin{array}{l}\text { GLY PI- } 803 \text { (PI } 701-16) \\
\text { GLY PP DISCH PRESS }\end{array}$ & $3-1$ & & & 65.75 PsI & $\pi$ \\
\hline $\begin{array}{l}\text { GLYTI-805 (TI-701-A } 13 \text { ) } \\
\text { GLY CIRC PUP SUCT TEMP }\end{array}$ & $3-17$ & & & $100-120 \mathrm{r}$ & $\widetilde{\pi \theta}$ \\
\hline 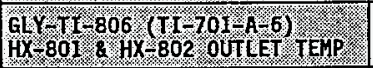 & $3-$ & & & $100-120 \%$ & \\
\hline 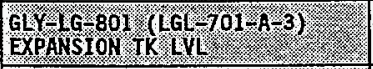 & $3-17$ & 1533 & & $1 / 3=2 / 3 \cdot F$ & \\
\hline 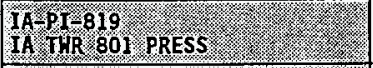 & $3-17$ & 1533 & & 80\% 105.951 & $\widehat{1 \theta}$ \\
\hline $\begin{array}{l}\text { IA PI } 820 \text {. } \\
\text { IA PURGE PRESS }\end{array}$ & $3-17$ & & & 40. $60 . \mathrm{psI}$ & 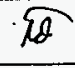 \\
\hline IA MT 818 , PRESS & $3-17$ & 15 & & $80.105 .9 S$ & $\sqrt{18}$ \\
\hline 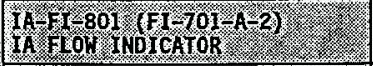 & $3-17$ & & & $45=90, \mathrm{CF} H$ & in \\
\hline 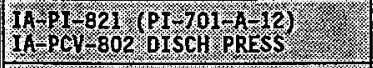 & $3-17$ & & & $30.45 .95 \%$ & $\mathscr{Q 0}$ \\
\hline 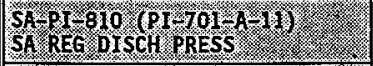 & $3-17$ & & & 25.45 .951 & 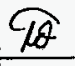 \\
\hline 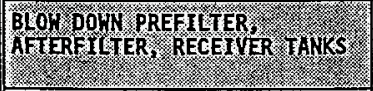 & $2-17$ & & & & \\
\hline 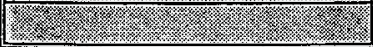 & & & & (1) & \\
\hline
\end{tabular}


HNF-SD-WM-OTR-231, Rev 0

Page 131 of 165

\section{WORKING COPY}

(1)

\section{8-HOUR ON-LINE TEST DATA SHEET (Cont.)}

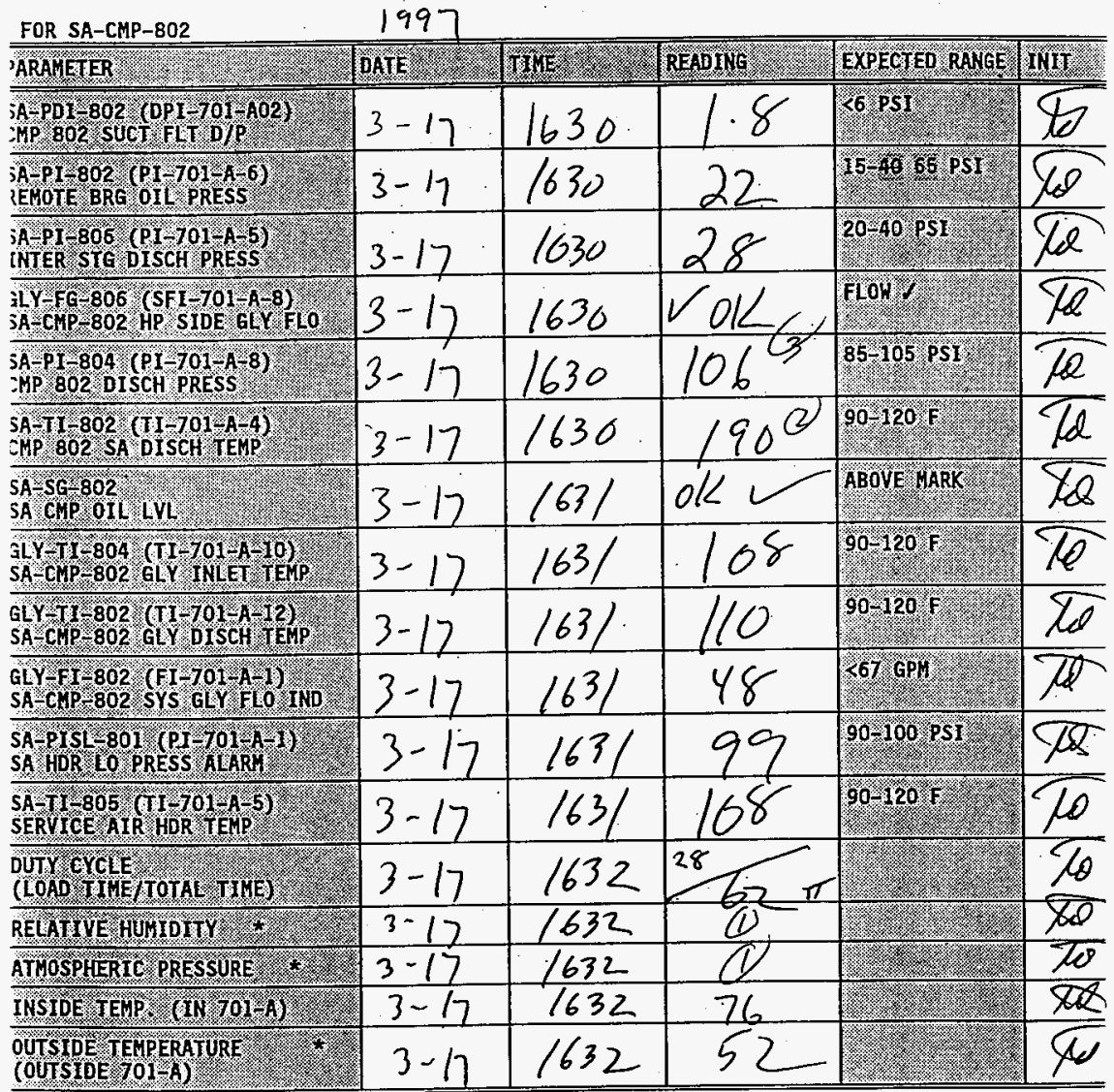

* Call weather station for required information at $373-2875$

Note: If readings are outside designated ranges, Notify Test Director.

s not out of spoc pop ot $P$

2 ses Los entre tydy

sespatis 10:70 
HNF-SD-WM-OTF-231, Rev 0

Page 132 of 165

\section{WORKING COPY}

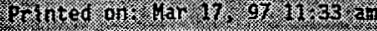

48-HOUR ON-LINE TEST DATA SHEET (Cont.)

\begin{tabular}{|c|c|c|c|c|c|}
\hline PARAMETER & DATE & ME: & READING & EXPECTED R & WII \\
\hline GLP PUIP RUMIIG , & $3-17$ & 1633 & & 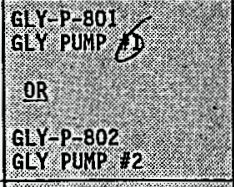 & \\
\hline 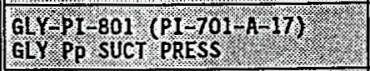 & $3-17$ & 1633 & $a$ & 0.10 PSI & 5 \\
\hline $\begin{array}{l}\text { GLYPI } 803 \text { (PI-701 }-A-16) \\
\text { GVY P DISCH RRESS }\end{array}$ & $3-17$ & $16^{33}$ & 6 & $65-75$ PSI & \\
\hline 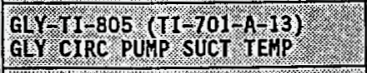 & $3 \cdot 17$ & 1633 & 110 & $100-120$ & \\
\hline 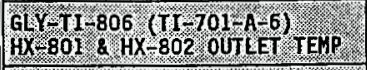 & $3-17$ & 1633 & 110 & $160-120 \%$ & \\
\hline 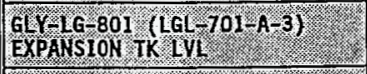 & $3-17$ & $16^{33}$ & $2 / 3$ & $1 / 3-2 / 3.70$ & Do \\
\hline $\begin{array}{l}\text { IA-PI- } 819 \\
\text { IA THR } 801 \text { PRESS }\end{array}$ & $3-17$ & 1633 & 90 & $80-105 . \mathrm{psI}$ & to \\
\hline 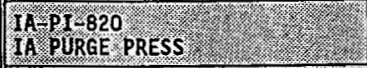 & $3-17$ & 1634 & 42 & $40-60.951$ & \\
\hline $\begin{array}{l}\text { MART } 818 \text { I } \\
\text { IA ThR } 802 \text { PRESS }\end{array}$ & $3 \cdot 17$ & 1634 & $\theta$ & $80-105$. 951 & \\
\hline 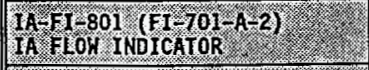 & $3-17$ & $16^{34}$ & 20 & $45-90 \mathrm{CF}$ & \\
\hline 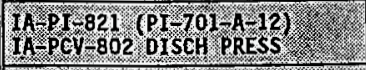 & $3-17$ & $163+$ & 2 & 30,4 & \\
\hline 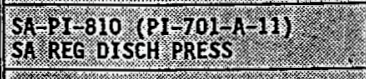 & $3-17$ & $16^{34}$ & 39 & $25-45 \cdot \mathrm{ps} 1$ & ) \\
\hline 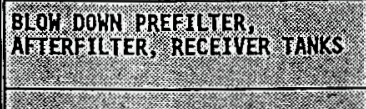 & 317 & 434 & & & bo \\
\hline 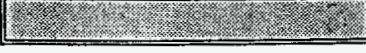 & & & & 20 & \\
\hline
\end{tabular}


HNF-SD-WM-OTR-231, Rev 0

Page 133 of 165

\section{WORKING COPY}

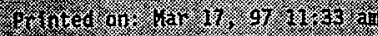

\section{8-HOUR ON-LINE TEST DATA SHEET (cont.)}

\begin{tabular}{|c|c|c|c|c|c|}
\hline HABQETER & (3) & What & KLAG & EXPECTED RAGE & INIT \\
\hline 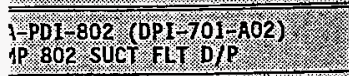 & $3-$ & 1633 & & & to \\
\hline 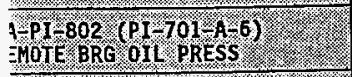 & $3-17$ & 1037 & 2 & $15=496$ & to \\
\hline $\begin{array}{l}\text { PPT } 806 \text { (PI } 701-415) \\
\text { ITER STG DISCH PRESS }\end{array}$ & $3-17$ & $163\rangle$ & & $20-$ & 80 \\
\hline $\begin{array}{l}\text { YYFG-806 (SFI-7O1-A } 8 \text { ) } \\
\text { A-CHP-802 HP SIDE GLY FLO }\end{array}$ & $3-17$ & 1633 & & Floh ? & 20 \\
\hline 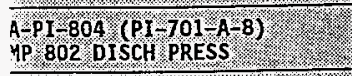 & $3-17$ & 1633 & & $85-105$ & \\
\hline 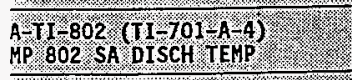 & $3-17$ & 1634 & & $90-120$ & \\
\hline $\begin{array}{l}\text { A-SG } 802 \\
\text { A CFP } 01 L ~ L V L\end{array}$ & $3-17$ & 1634 & & ABOy & $\overline{4}$ \\
\hline 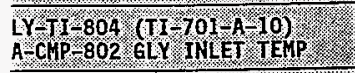 & $3-17$ & 1634 & & $90-120$ & 80 \\
\hline 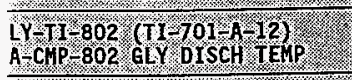 & $3-17$ & 1634 & h & $90-120$ & $\pi$ \\
\hline 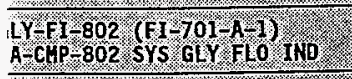 & $3-17$ & 634 & & & 6 \\
\hline $\begin{array}{l}\text { A-PISL } 801 \text { (PI TO1/A) I) } \\
\text { A HDR IO PRESS ALARH }\end{array}$ & $3-17$ & 634 & & & $\$ 8$ \\
\hline 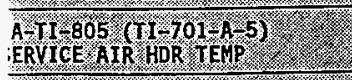 & $3-72$ & 1634 & & & 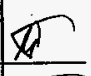 \\
\hline 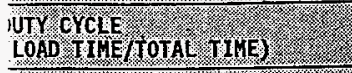 & $3-17$ & & & & \\
\hline EATIVE HUMIDHY * * & $3-17$ & 1635 & $\overline{0}$ & & $\Phi$ \\
\hline THOSPHERIC PRESSURE T TV & $3-17$ & 1636 & (1) & & \\
\hline NSIDE TEMP (IN $701-\mathrm{A})$ & $3-17$ & 6665 & 70 & & 电 \\
\hline $\begin{array}{l}\text { UTSIDE IENERATURE } \\
\text { OUTSIDE } 701 \text { - A) }\end{array}$ & $3-$ & (1) 2h & T大 & & A \\
\hline
\end{tabular}

* Call weather station for required information at 373-2875

Note: If readings are outside designated ranges, Notify Test Director.

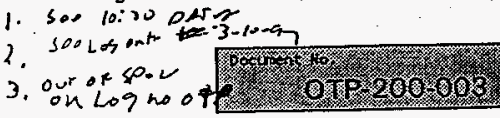


HNF-SD-WM-OTR-231, Rev 0

Page 134 of 165

\section{WORKINE COPY}

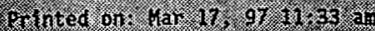

48-HOUR ON-LINE TEST DATA SHEET (Cont.)

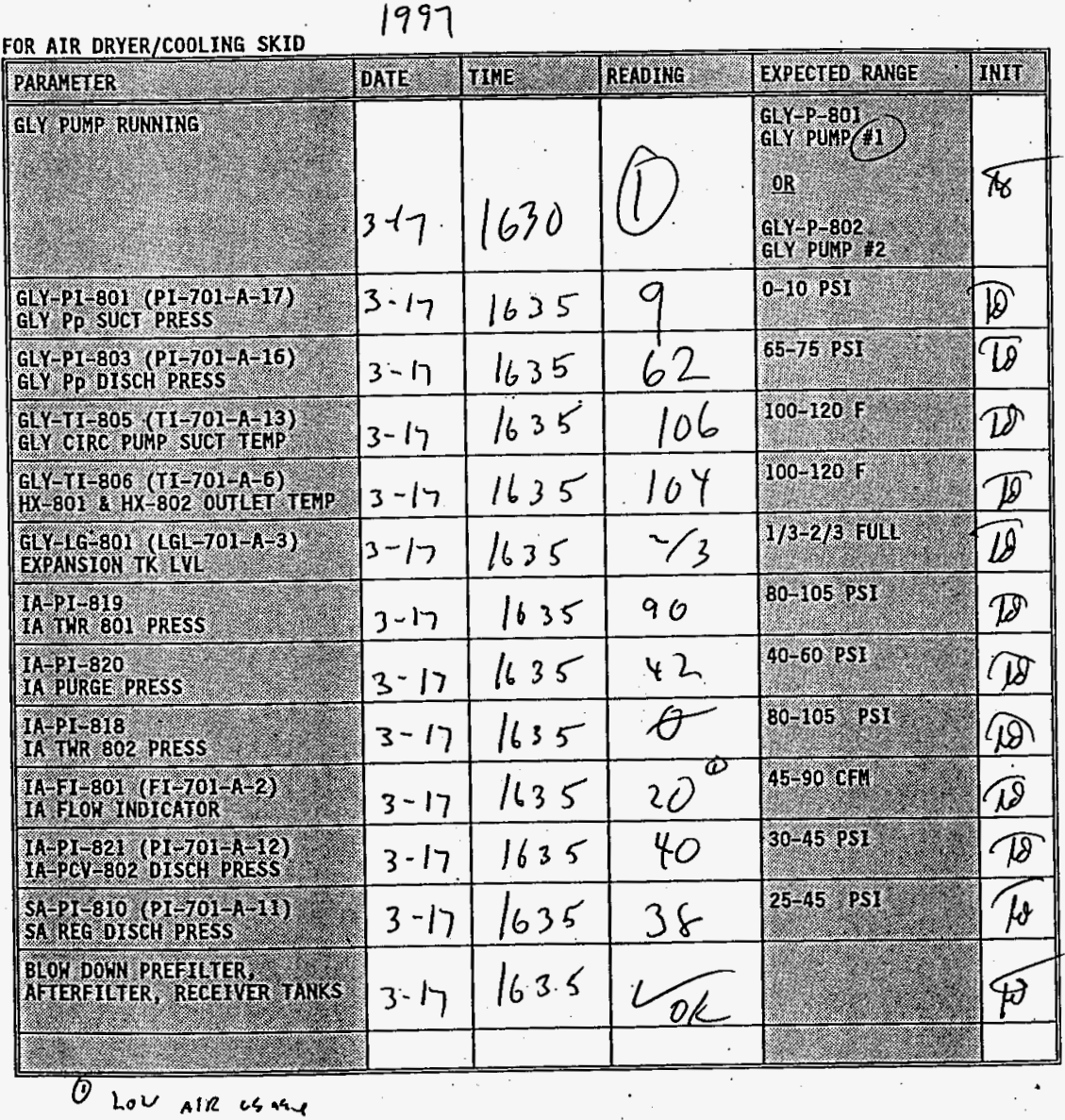


HNF-SD-WM-OTR-231, Rev 0

Page 135 of 165

\section{WORKING COPY}

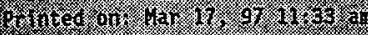

\section{8-HOUR ON-LINE TEST DATA SHEET (Cont.)}

\begin{tabular}{|c|c|c|c|c|c|}
\hline ANETER & Date & 158 & REAQRN & EXPETED RANGE & 1Nit \\
\hline 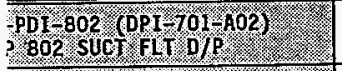 & $3-17$ & 1730 & & 6.851 & tre \\
\hline $\begin{array}{l}\text { PI } 102 \text { (PI } 701 \text { A } 6) \text { ) } \\
\text { TOTE BRG OIL RRESS }\end{array}$ & $3-17$ & 1730 & 2 & $15 \% 6 \%$ & Tx \\
\hline 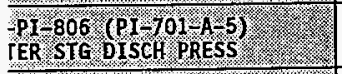 & $3-17$ & 1731 & & $20=4$ & $T x$ \\
\hline 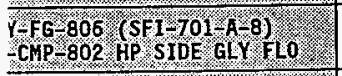 & $3-17$ & 1731 & & $\mathrm{FLOH}$ & $T x$ \\
\hline 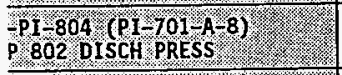 & $3-17$ & 1732 & 13 & 85-105 PSI & $7 x$ \\
\hline 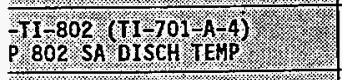 & $3-17$ & 1732 & $+8 \Rightarrow \frac{1903}{n_{c}}$ & $90-120 \%$ & $7 \pi$ \\
\hline $\begin{array}{l}\mathrm{SG} 802 \\
\mathrm{CHP} 0 \mathrm{IL} / \mathrm{VL}\end{array}$ & $3-17$ & 1733 & & $A B O N$ & $T x$ \\
\hline 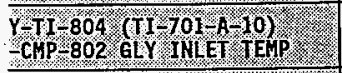 & $3-17$ & 1733 & & 90. & $\pi$ \\
\hline 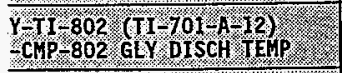 & $3-17$ & 1737 & & $90-120$ & $\mathbb{R}$ \\
\hline 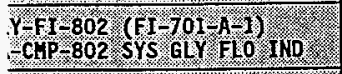 & $3-17$ & 1733 & & $67 \mathrm{GPI}$ & $T K$ \\
\hline 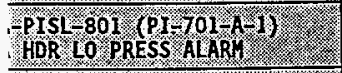 & $3-17$ & 1734 & 93 & & $7 x$ \\
\hline $\begin{array}{l}\text { TI } 805 \text { (TI TO1 A } 5 \text { ) } \\
\text { RUICE AIR YDR TEMP }\end{array}$ & $3-17$ & 1734 & 1.08 & 90 & TZ \\
\hline $\begin{array}{l}\text { TY CralE } \\
\text { OAD TIME TOTA TIF) }\end{array}$ & $3-17$ & 1734 & $25 \%$ & . & 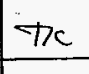 \\
\hline HIVE HUMIDI Y ( & $3 \cdot 17$ & & (a) & & \\
\hline MOSPHERIC PRESSURE & $3-17$ & $\cdot$ & (1) & & \\
\hline ISIDE $1 E \mathrm{EP} .(1 \mathrm{~N} / \mathrm{TO} 1-\mathrm{A})$ & $3-17$ & 1729 & 7.8 & & $7 k$ \\
\hline $\begin{array}{l}\text { ITSIDE TEMPERTURE } \\
\text { UTSIDE } 7.01-\mathrm{A} \text { ) }\end{array}$ & $3-17$ & 1728 & 50 & ?. & $\mathbb{Z}$ \\
\hline
\end{tabular}

* Call weather station for required information at $373-2875$

Note: If readings are outside designated ranges, Notify Test Director.

Ser osta $b: \mathrm{W}$

ses Los antry $3 / 10 / 97$

sut of spoc on lo s 
HNF-SD-WM-OTR-231, Rev 0

Page 136 of 165

\section{WORKING COPY}

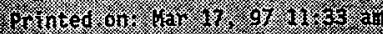

48-HOUR ON-LINEE TEST DATA SHEET (cont.)

OOR AIR DRYER/COOLING SKID

\begin{tabular}{|c|c|c|c|c|c|}
\hline PARALEIER & DATE & $1 \mathrm{WL}$ & REDDING & EXPECTEO RANGE & WH \\
\hline 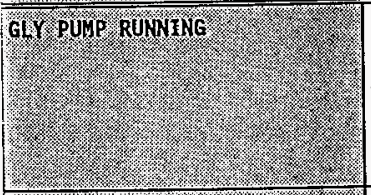 & 317 & 1737 & & 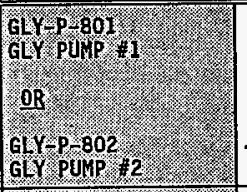 & TK \\
\hline 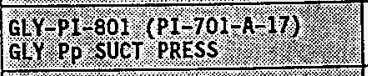 & $3-17$ & 1737 & 10 & O-10 PSI & Th \\
\hline $\begin{array}{l}\text { GLYPI } 803 \text { (PI-701-A-16) } \\
\text { GLY PP DISCH PRESS }\end{array}$ & $3-17$ & 1738 & 67 & $65-75$ PSI & 17 \\
\hline $\begin{array}{l}\text { GLYTTI } 805 \text { (II } 701 \text { A } 13 \text { ) } \\
\text { GLY CIRC PUMP SUCT TEMP. }\end{array}$ & $3-17$ & 1238 & 2 & $100=120$ & T \\
\hline 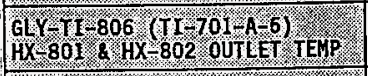 & $3-17$ & 1738 & & $100-120 \mathrm{~F}$ & $T$ \\
\hline 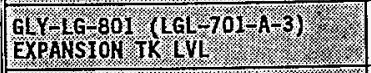 & $3-17$ & 1738 & & $1 / 3-2 / 3$ Fu & \\
\hline $\begin{array}{ll}\text { IA PI } 819 \\
\text { IA THR 80I PRESS }\end{array}$ & $3-17$ & 1736 & 100 & $80-105$ PSI & \\
\hline IA PURGE PRESS & $3-17$ & 1736 & 0 & & $7 K$ \\
\hline $\begin{array}{l}\text { IA PT } 1818 \text {, } \\
\text { IA THR } 802 \text { PRESS }\end{array}$ & $3-17$ & 1736 & 104 & $80-105 \cdot \mathrm{PS}$ & \\
\hline 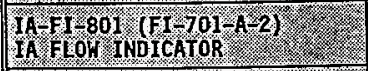 & $3-17$ & 1739 & & & \\
\hline 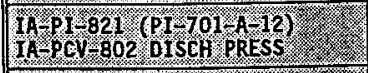 & $3-17$ & 1739 & 47 & $30=45$ PSI & \\
\hline 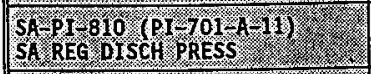 & $3-17$ & 1739 & 39 & $25 \div 45 \cdot P S 1$ & \\
\hline 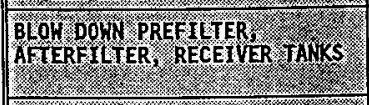 & $3-i 7$ & $x^{1740}$ & & 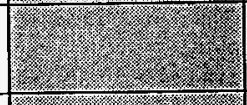 & $x$ \\
\hline \begin{tabular}{|l|l|l|l|l|l|l|l|}
. & \\
\end{tabular} & & & & 1.2. & \\
\hline
\end{tabular}

Q Lov air cSate 
HNF-SD-WM-OTR-231, Rev O

Page 137 of 165

WORKING CORX

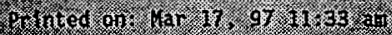

\section{8-HOUR ON-LINE TEST DATA SHEET (Cont.)}

\begin{tabular}{|c|c|c|c|c|c|}
\hline AMETER & DAlf & $16 \mathrm{E}=2$ & BEADING & EXPECT EO RANGE & INI \\
\hline 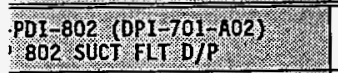 & $3-h$ & 1730 & 1.8 & $66 \mathrm{PSI}$ & 80 \\
\hline 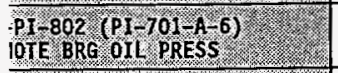 & $3-17$ & & 20 & $15-668$ & 80 \\
\hline $\begin{array}{l}\text { PI Bof (PI LO1-A S S) } \\
\text { ER STG DISCH PRES }\end{array}$ & $3-h$ & & 28 & $20-49$ ? & 40 \\
\hline 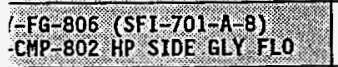 & $3-17$ & & & 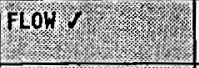 & 60 \\
\hline $\begin{array}{l}\text { PI } \\
\text { P } 802 \text { O DISCH PRESS } \\
\end{array}$ & 3.7 & & 106 & $85-105$ PS & 40 \\
\hline 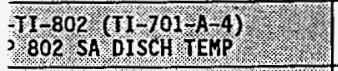 & $3-17$ & & 186 & $90-120=$ & tel \\
\hline 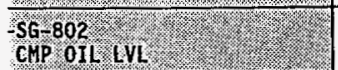 & $3-17$ & & & ABOVE MAR & 10 \\
\hline 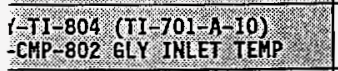 & $3 \cdot 17$ & & 1 & $90-120=$ & 18 \\
\hline 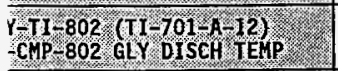 & $3-17$ & & & $90-120 \%$ & 10 \\
\hline 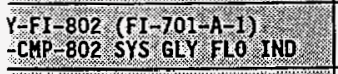 & $3-17$ & & & 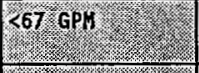 & 10 \\
\hline $\begin{array}{l}\text { PISU } \\
\text { HDR LO PRESS PILARI) }\end{array}$ & $3-17$ & & & & 78 \\
\hline 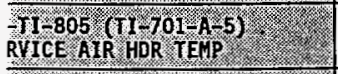 & $3-17$ & & & & 20 \\
\hline 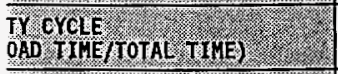 & $3-12$ & & & & $\sqrt{4}$ \\
\hline 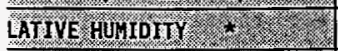 & $3-17$ & & (1) & & $\bar{N}$ \\
\hline 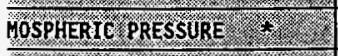 & 3.17 & 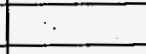 & 0 & & 5 \\
\hline SIDE IEA $($ IN 701 A) & $3-17$ & & 72 & & ge \\
\hline $\begin{array}{l}\text { TSIOE TEPERATRE } \\
\text { TTSTDE } 01-A \text { ) }\end{array}$ & $3-12$ & & & & \\
\hline
\end{tabular}

- Call weather station for required information at 373-2875

lote: If readings are outside designated ranges, Notify Test Director.

1. Sre 10:70 DAtA

2 see Log 3-6097

3 out of spec on 
HNF-SD-WM-OTF-231, Rev 0

Page 138 of 165

\section{WORKING COPY}

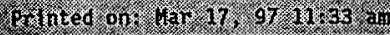

\section{8-HOUR ON-LINEE TEST DATA SHEET (cont.)}

FOR AIR DRYER/COOLING SKID

1997

\begin{tabular}{|c|c|c|c|c|c|}
\hline PARAME ER , , & DATE & $714 \%$ & RE ADING & EXPETEO RAMGE & $\mathrm{KH}_{\mathrm{H}}$ \\
\hline GP PUM RUMING & $3-17$ & & & 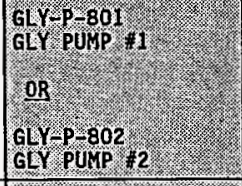 & \\
\hline 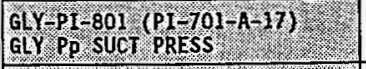 & $3-17$ & & & $0.10 . \mathrm{pST}$ & \\
\hline GLY PI-803 (PI-701-A-16) & $3-17$ & & & 65.75 .951 & E) \\
\hline 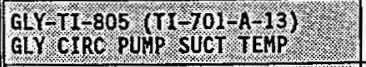 & $3-17$ & & & $100-120$ & 8 \\
\hline 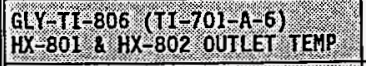 & $3 \cdot 17$ & & & $100-120 \%$ & 170 \\
\hline 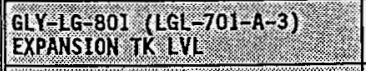 & $3-17$ & & & $1 / 3-2 / 3.7011$ & $D$ \\
\hline $\begin{array}{l}\text { IAPI } 819 \\
\text { IA TWR } 801 \text { PRESS }\end{array}$ & $3-17$ & & & $80-105 \mathrm{PSI}$ & \\
\hline $\begin{array}{l}\text { IAPI } 820 \\
\text { IA PURE PRESS }\end{array}$ & $3 \cdot 17$ & & & $40-60 . p S T$ & \\
\hline $\begin{array}{l}\text { TAPI } 818 \\
\text { IA TWR } 80 \text { 2 PRESS }\end{array}$ & 3.17 & & & $80-105: 951$ & \\
\hline 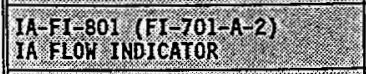 & $3 \cdot 17$ & & 2 & $45-90.954$ & \\
\hline 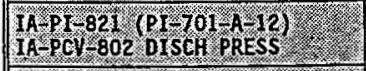 & $3 \cdot 17$ & & & $30=75$ PSI & $\sqrt{10}$ \\
\hline 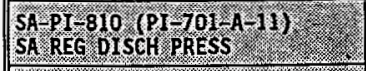 & $3-1$ & & & $25.45 .9 \mathrm{PSI}$ & 40 \\
\hline 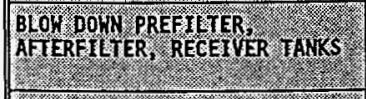 & $3 \cdot 17$ & & & & \\
\hline lर & & & & $\sqrt{3.8}$ & \\
\hline
\end{tabular}


HNF-SD-WM-OTR-231, Rev 0

Page 139 of 165

\section{WORKING COPY}

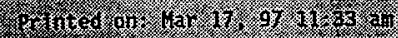

\section{8-HOUR ON-LINE TEST DATA SHEET (Cont.)}

\begin{tabular}{|c|c|c|c|c|c|}
\hline ANETER & DAIE & $\mathrm{MME}$ & READING & EXPEAIED RAMGE & 14 \\
\hline 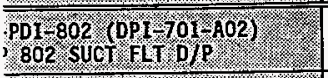 & $3-17-9\rangle$ & $\begin{array}{l}2327 \\
-138.9 \mathrm{~m}\end{array}$ & 2.0 & 6. prst & $D_{4}$ \\
\hline $\begin{array}{l}\text { P1 } 802 \text { (PI } 701 \text { A } 6 \text { ) } \\
\text { POTE BRG OIL PRESS }\end{array}$ & $3 \cdot 12-97$ & 2327 & 20 & 15- 65 & Da \\
\hline $\begin{array}{l}\text { PI-806 (PI-701-1) } 5) \\
\text { ER STO DISCH PRESS }\end{array}$ & $3-17-91$ & 2328 & 29 & 20-40.PSI & an \\
\hline 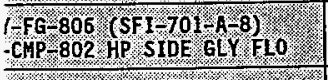 & $3 \cdot 17-97$ & 2328 & & FLon & \\
\hline $\begin{array}{l}\text { PI 804 (PI 701-A }-8) \\
802 \text { DISCH PRESS }\end{array}$ & $3 \cdot 17-97$ & 2328 & 1100 & $85 \cdot 105 . p$ & 4 \\
\hline 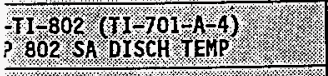 & $3-17-97$ & 2328 & (2) & 90.120 & \\
\hline $\begin{array}{l}\text { SG } 802 \\
\text { CHP OIL } 1 \mathrm{VL}\end{array}$ & $3-17-9)$ & 2329 & & ABOVE Y & \\
\hline $\begin{array}{l}\text { TI-804 (TI TO1 A } 10) \\
\text { CHP-802 GLY INLT TEMP }\end{array}$ & $3-17-97$ & 2329 & 98 & $90-120.0$ & \\
\hline $\begin{array}{l}\mathrm{Y}_{\mathrm{T}} \mathrm{T}_{1}-802 \\
\text { CHP }\end{array}$ & $3-17-97$ & 2329 & 99 & $90=120 \mathrm{C}$ & \\
\hline 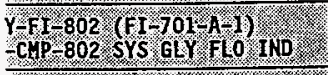 & $3.17-97$ & 2329 & 50 & $67.6 P H$ & $D_{L}$ \\
\hline $\begin{array}{l}\text { PISL } 801 \text { (PI-70I-A }-1) \\
H D R=10 \text { PRESS A ARH }\end{array}$ & $3 \cdot 17 \cdot 97$ & 2330 & 9 & $90-100$ PST & $D_{n}$ \\
\hline 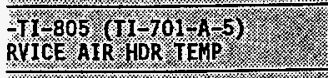 & $3-17-97$ & 2327 & $T$ & $90-120$ & W \\
\hline $\begin{array}{l}\text { TYCYCLE } \\
\text { OAD T IME TOTAL T TIIS) }\end{array}$ & $3-12 \cdot 97$ & 2327 & $25 \times$ & & \\
\hline WTIV HUMIOMI & $3-17-27$ & & & & . \\
\hline BOSPHERIC QRESSURE & $3.17-97$ & & 0 & & $D_{n}$ \\
\hline SIDE T TEM ( (IN $201-\mathrm{A})$ & $3-17-97$ & 2327 & 70 & & $D_{n}$ \\
\hline $\begin{array}{l}\text { TSTDE TEWPERATURE } \\
\text { UTSIDE }(0)=\text { ( })\end{array}$ & $3-12-97$ & 2332 & 46 & & Dh \\
\hline
\end{tabular}

- Call weather station for required information at $373-2875$

lote: If readings are outside designated ranges, Notify Test Director.

1. See 10:30 DAt $\Omega$

2. See Los B oolk entry 3-10-97

3 out of spoc

on pata sncet

net at she

con, nat... in 
HNF-SD-WM-OTR-231, Rev 0

Page 140 of 165

WORKINE COPY

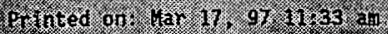

48-HOUR ON-LINE TEST DATA SHEET (Cont.)

FGR AIR DRYER/COOLING SKID

\begin{tabular}{|c|c|c|c|c|c|}
\hline PARAMELR & BATE & $\min E$ & KREAJNG & EXPECTED RANGE & $1 \mathrm{hu}$ \\
\hline 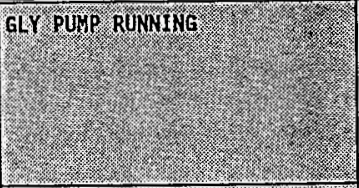 & $3-n-97$ & $\frac{2320}{4+30}$ & $\theta$ & 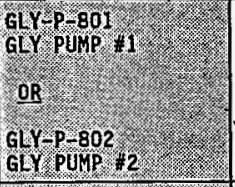 & \\
\hline $\begin{array}{l}\text { GL }-P I-801 \\
\text { GL }\end{array}$ & $3 \cdot 17 \cdot 9$ & 2330 & & 0-10. $\mathrm{p}$ & \\
\hline $\begin{array}{l}\text { GLYPI } 803 \text { (PI } 701-\mathrm{A}-16) \\
\text { GLYPP DISCH PRES }\end{array}$ & $3-17-9)$ & 2330 & & 65.75 PSI & \\
\hline 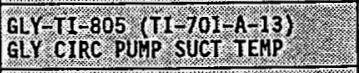 & $3-17-97$ & 2330 & & $100-120$ & \\
\hline 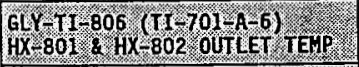 & $3-17-87$ & 2331 & & $100-120$ & \\
\hline 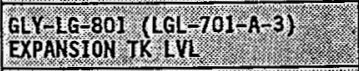 & $3-1297$ & 2331 & & $1 / 3-2 / 3$ & \\
\hline IA PT-819 & $6 \cdot 17 \cdot 97$ & 2332 & & 80.105 & \\
\hline $\begin{array}{l}\text { IAPIR820 } \\
\text { IA PURGE PRESS }\end{array}$ & $3 \cdot 17 \cdot 97$ & 2332 & & 80-60. & \\
\hline 19P P 818 \% & $3-12-97$ & 2332 & & 80.105 .85 & \\
\hline 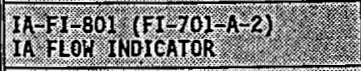 & $p \cdot n \cdot 97$ & 2331 & 20 & $45-90005$ & \\
\hline 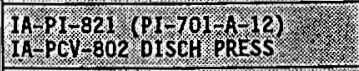 & $3 \cdot 17 \cdot 97$ & 2331 & & $30=45851$ & \\
\hline 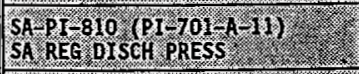 & $3-17-97$ & 2331 & 40 & 25.75. PSI & \\
\hline 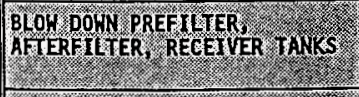 & $3-17$ & 2332 & & & \\
\hline (1) & & & & 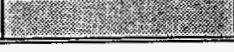 & \\
\hline
\end{tabular}

(1) Low sin usise 
HNF-SD-WM-OTR-231, Rev 0

Page 141 of 165

\section{WORKING COPY}

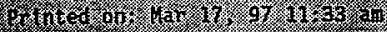

48-HOUR ON-LINE TEST DATA SHEET (Cont.)

\begin{tabular}{|c|c|c|c|c|c|}
\hline URALTER & DATE & TINE & REAPIMG & EXPECTED & 1111 \\
\hline 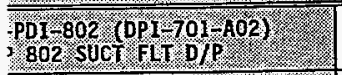 & $3 / 18 / 97$ & 0328 & 2.0 & $6.8 \mathrm{PI}$ & \\
\hline 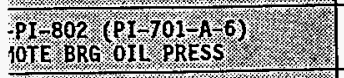 & $3 / 18 / 97$ & 0328 & 22 & $15-6.6$ & \\
\hline $\begin{array}{l}\text { PI-806 (PI } 701-A=5) \\
\text { TER SIG DISCH PRESS }\end{array}$ & $3 / 18 / 97$ & 0329 & 29 & $20=40.9$ & \\
\hline 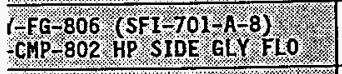 & $3 / 18 / 97$ & 0329 & & $\mathrm{FLOH} /$ & \\
\hline $\begin{array}{l}\text { P1-804 (PI-701-A } 8) \\
\text { 302 DISCH PRESS }\end{array}$ & $3 / 18 / 97$ & 0330 & 110 & $85-105$ & \\
\hline $\begin{array}{l}\text { TI-802 (TI TO1-A A) } \\
\text { P } 802 \text { SA DISCH TEMP }\end{array}$ & $3 / 18 / 97$ & 0330 & 171 (3) & $90-120.1$ & \\
\hline $\begin{array}{l}\text { SG } 802 \\
\text { CMP } 011.4 \mathrm{VL}\end{array}$ & $3 / 18 / 97$ & 0330 & & ABOYE. M & \\
\hline 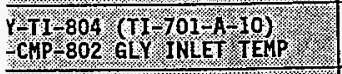 & $3 / 18 / 97$ & 0330 & 100 & $90-120 \%$ & Din \\
\hline 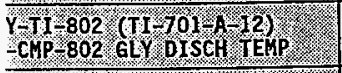 & $3 / 18 / 97$ & 0330 & 102 & $90-120 \mathrm{~F}$ & $D_{h}$ \\
\hline 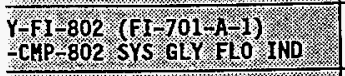 & $\sqrt[3]{18} / 17$ & 0,331 & & 67. GPY & 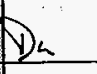 \\
\hline 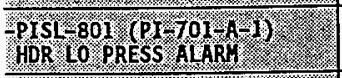 & $3 / 1 / 47$ & 0331 & 100 & $90 \times 100 \mathrm{ps}$ & \\
\hline $\begin{array}{l}\text { TI } 805 \text { (TI } 701-1-5) \\
\text { RVICE AR HDR TEMP }\end{array}$ & $8 / 15 / 97$ & 0328 & 100 & $90-120.9$ & 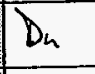 \\
\hline $\begin{array}{l}\text { TY cYcLE } \\
\text { OAD THETOTAL TIME) }\end{array}$ & $3 / 18$ & .0321 & $26 \times 1 / 63 \mathrm{sec}$ & & \\
\hline WTIV HUMIDIY : & $3 / 18 / 97$ & & & & \\
\hline MOSPHERTC PRESSURE & $\sqrt[3]{19} / 97$ & & (1) & & 4 \\
\hline 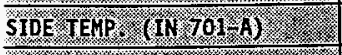 & $8 / 18 / 19$ & 0331 & $72^{\circ}$ & & $D n$ \\
\hline $\begin{array}{l}\text { ISIDE TEMPERTURE } \\
\text { UTSIDE TOI AS }\end{array}$ & $3 / 12 / 17$ & 0331 & $44^{\circ}$ & \% & \\
\hline
\end{tabular}

* Call weather station for required information at $373-2875$

lote: If readings are outside designated ranges, Notify Test Director.

See 1030 DAFr $3 / 17 / 97$

see loginose rentry $3 / 10 k 7$ - 1940 thes.

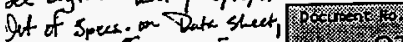

Jot out of spees for

19. 20.30 
HNF-SD-WM-OTR-231, Rev 0

Page 142 of 165

\section{WORKINE COPY}

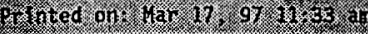

\section{8-HOUR ON-LINE TEST DATA SHEET (Cont.)}

FOR AIR DRYER/COOLING SKID

\begin{tabular}{|c|c|c|c|c|c|}
\hline PRRAVIER & DATE & TIV & READING & EXPEOTED RANGE & WIT \\
\hline $\mathrm{GLT}$ PUIP RUNING & $3 / 1 8 \longdiv { 9 7 }$ & 0331 & 80 & 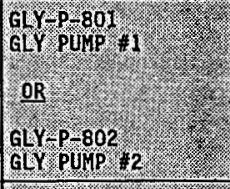 & \\
\hline GLY PI 801 (PI-701-A. 17 ) & $3 / 1$ & 2332 & $?$ & $0=10 \mathrm{PSI}$ & \\
\hline 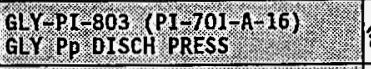 & $3 / 18$ & 0332 & & $65-75.951$ & \\
\hline 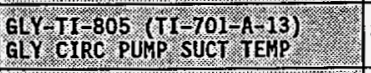 & $3 / 18$ & 0332 & 102 & $100-120 \%$ & \\
\hline 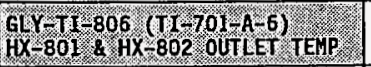 & & $\cos 32$ & 108 & $100-120 \%$ & \\
\hline 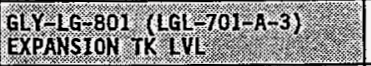 & $8 / 18 / 97$ & 0332 & & $1 / 3-2 / 3,70$ & \\
\hline 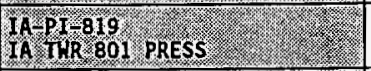 & & $D 332$ & & $80-105 \mathrm{PSI}$ & \\
\hline IA PI RTER PRESS & $3 / 18 / 1$ & 0332 & 4 & $40=60.951$ & \\
\hline YA PI 818 TH 8 PRES & & .332 & & $80=105 \cdot \mathrm{ps}$ & \\
\hline 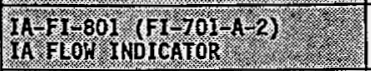 & $3 / 18 /$ & 6333 & 200 & $45-90 \mathrm{ccm}$ & \\
\hline 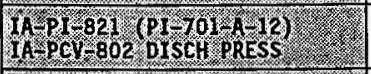 & $3 / 18$ & 0333 & 42 & $30-45.951$ & 从 \\
\hline 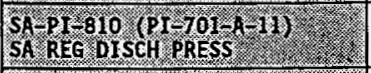 & $3 / 16 / 17$ & 0333 & 40 & $25 \div 45 \times$ PSI & 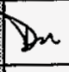 \\
\hline 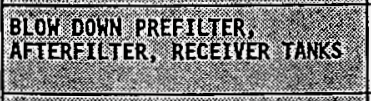 & $3 / 18 / 97$ & 0333 & & & 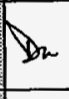 \\
\hline 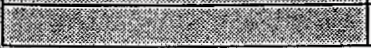 & & & & 2.8. & \\
\hline
\end{tabular}

(1) Low Air Usage. 
HNF-SD-WM-OTR-231, Rev 0

Page 143 of 165

\section{WORKINC COPY}

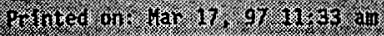

48-HOUR ON-LINE TEST DATA SHEET (Cont.)

\begin{tabular}{|c|c|c|c|c|c|}
\hline RAMETER & DATE & IMI & PEADNG & EXPETEO RANGE & $\mathrm{INR}$ \\
\hline 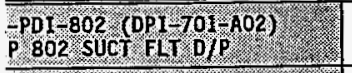 & $3 / 18 / 97$ & .726 & 2.0 & 26.851 & \\
\hline $\begin{array}{l}\text { PI-802 (PITIOI A } 6) \\
\text { MOTE BRG } 01 \text { PRESS }\end{array}$ & $3 / 18$ lar & .726 & 22 & $15-465$ & \\
\hline $\begin{array}{l}\text { PI } 806 \text { (PI 701 A A 5) } \\
\text { TER STG DISCH PRESS }\end{array}$ & $3 / 18 / 97$ & 0727 & 29 & $200 \%$ & \\
\hline 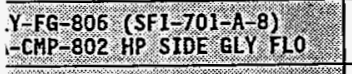 & $3 / 18 / 97$ & b727. & & Flow & \\
\hline $\begin{array}{l}\text { PI- 804 (PI-701-A } 8 \text { ) } \\
\text { IP } 802 \text { DISCH PRESS }\end{array}$ & $8 / 18 / 97$ & $b 727$ & (2) & $85-10$ & \\
\hline $\begin{array}{l}\text { T1-802 (TI-701 A-4) } \\
\text { P } 802 \text { SA DISCH TEMP }\end{array}$ & $3 / 18 / 97$ & 0727 & (1) & & \\
\hline $\begin{array}{l}\text { SG } 802 \\
\text { CMP } 01 \mathrm{LVV} .\end{array}$ & $3 / 18 / 87$ & .0727 & & ABOVE & \\
\hline 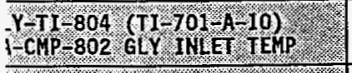 & $3 / 18 / 17$ & 0728 & 100 & $90-120$ & \\
\hline 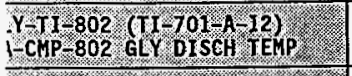 & $3 / 18 / 97$ & 0728 & 101 & & \\
\hline 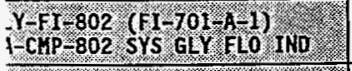 & $3 / 18 / 97$ & 0728 & 50 & $367 \mathrm{GPH}$ & \\
\hline 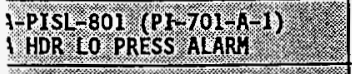 & $3 / 18 / 97$ & .728 & 99 & $90=100$ & \\
\hline 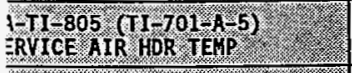 & $3 / 18 / 97$ & 0726 & & & \\
\hline $\begin{array}{l}\text { JYY Grcie } \\
\text { OAD TIHE LOTAL TINE) }\end{array}$ & $3 / 18 / 97$ & 0224 & $24 / \operatorname{cosscc}$ s. & & \\
\hline HAT Y E HUHIOHY & $3 / 18 / 17$ & 6724 & onoi Recaxin & & De \\
\hline TMOSPHERIC RRESSURE - > & $3 / 18 / 97$ & .2724 & $20.12 \quad 2$ & & \\
\hline 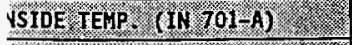 & $3 / 18 / 17$ & 6731 & ting & & Du \\
\hline $\begin{array}{l}\text { TS IDE TEMPERAURE } \\
\text { JUTSIDE } 701 \% \text { S }\end{array}$ & $3 / 18 / 17$ & 6724 & $.700^{\text {The }}$ lecording & 2. & \\
\hline
\end{tabular}

- Call weather station for required information at 373-2875

Note: If readings are outside designated ranges, Notify Test Director.

ec $\log$ Book Entry 3iskat-1940 Hes.

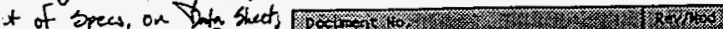

+ Qut of speci por Ote. 
FOR AIR DRYER/COOLING SKID

\begin{tabular}{|c|c|c|c|c|c|}
\hline PARAMIIER & DATE & WHE & READING & SXPCTED RANGE & 111 \\
\hline GLY PUNP RUNAING & $3 / 18 / 97$ & 0228 & 801 & 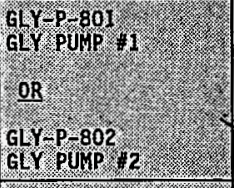 & \\
\hline $\begin{array}{l}\text { GY } / \text { PI-801 }(\mathrm{PI}-701-\mathrm{A}-17) \\
\text { GLY PP SUCT PRESS }\end{array}$ & $3 / 181$ & 0729 & & 0.10.9st & \\
\hline 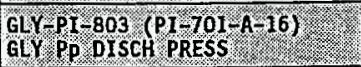 & $3 / 4 / 97$ & 0729 & 65 & $65-75.9 S 1$ & \\
\hline $\begin{array}{l}\text { GL T TY } \\
\text { GLY CIRC PUTP SUCT TEMP }\end{array}$ & $3 / 18 / 97$ & 0729 & 103 & $100=120 . r$ & \\
\hline 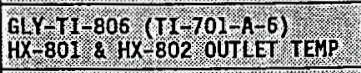 & $3 / 18 / 17$ & 0729 & 103 & $100-120 \%$ & \\
\hline 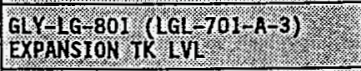 & $3 / 18 / 27$ & 0729 & & $13.2 / 3 . \mathrm{YUL}$ & \\
\hline 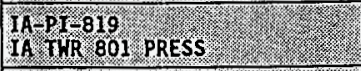 & $3 / 18 / 97$ & 0729 & & BO- 105.951 & \\
\hline $\begin{array}{l}1 \mathrm{~A} \mathrm{PI}_{\mathrm{O}} 82 \mathrm{O} \\
\mathrm{IA} \text { PURGE PRES }\end{array}$ & $3 / 18 / 17$ & 0729 & 4 & $40=60.951$ & \\
\hline $\begin{array}{l}\text { IA } \mathrm{p} \mathrm{T}-818 \\
\mathrm{IA} \text { THR } 802 \text { PRES }\end{array}$ & $4 / 18 / 97$ & .729 & 103 & $80-105 \div 2$ & \\
\hline 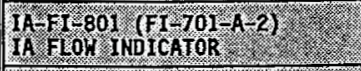 & $3 / 18 / 77$ & 0730 & 20 & $45=90.67$ & \\
\hline 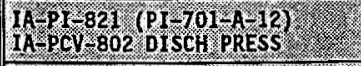 & $3 / 18 \% 97$ & 0750 & 42 & $30-45.9 S I$ & \\
\hline 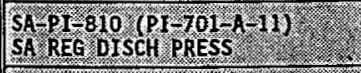 & $3 / 18 / 97$ & 0730 & 40 & $25-45 \%$ PS1 & \\
\hline 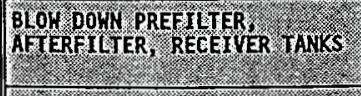 & $3 / 18 / 97$ & 0730 & & & \\
\hline L, & & & & 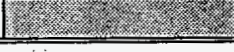 & \\
\hline
\end{tabular}




\section{8-HOUR ON-LINE TEST DATA SHEET (Cont.)}

=OR SA-CHP -802

\begin{tabular}{|c|c|c|c|c|c|}
\hline RAHETER & DATE & $\mathrm{MLE}$ & REPBING & EXPECTEO RANGE & 1Nu \\
\hline 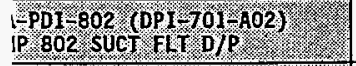 & $3 / 18 / 97$ & 1130 & 8 & K6.95T & TK \\
\hline 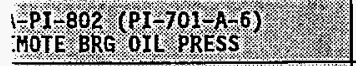 & $3 / 18 / 97$ & 1130 & 22 & $15-46.60$ & DK \\
\hline $\begin{array}{l}\text { PPI } 806(\text { PI } 701-4-5) \\
\text { TER STG DISCH RRESS }\end{array}$ & $3 / 18 / 97$ & 1.32 & & $20-40 . \mathrm{PS}$ & the \\
\hline 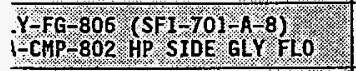 & $3 / 18 / 17$ & 1130 & & FlOH/ & $\pi c$ \\
\hline $\begin{array}{l}\text { PP1 804 (PI 701 A } 8) \\
\text { P 802 DISCH PRESS }\end{array}$ & $3 / 18 / 27$ & 1,31 & (3) & $85-105$ & $7 k$ \\
\hline 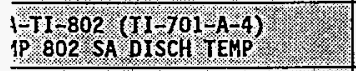 & $3 / 18 / 97$ & 131 & 178 & & $\pi S$ \\
\hline $\begin{array}{l}\text { 1-sG } 802 \\
\text { chP OII LVL }\end{array}$ & $3 / 18 / 47$ & 1130 & & ABOVE & $\pi x$ \\
\hline 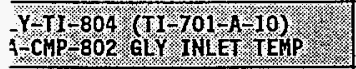 & & 1131 & & $90-120$ & iic \\
\hline 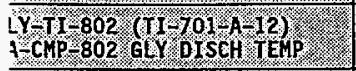 & $3 / 18 / 97$ & 1131 & & $90-120$ & $\pi x$ \\
\hline 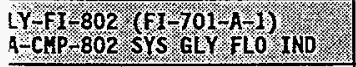 & $3 / 18 / 47$ & 1131 & & $367 \mathrm{GPH}$ & Th \\
\hline 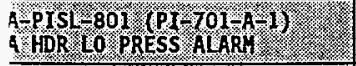 & $3 / 18$ & 1132 & 地 100 & $90=100$ & TK \\
\hline 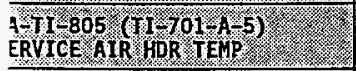 & $3 / 18 / 97$ & 1130 & 108 & $90.120 \%$ & tc \\
\hline $\begin{array}{l}\text { UTY CrCLE } \\
\text { CAD TIM TOLA TINE) }\end{array}$ & $3 / 12 / 97$ & 1.130 & $29^{\mathrm{sk}} / 63^{\mathrm{T}} \mathrm{sec}$ & & 㴶 \\
\hline ELTIY HUIDIY & $3 / 18 / 17$ & & (1) & & \\
\hline TWOSPHERIC RRESSURE & $3 / 18 / 17$ & & (D) & 13 & \\
\hline 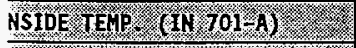 & $.3 / 18 / 97$ & $1+\frac{\pi x}{32} 1132$ & 71 & (2) & $\pi k$ \\
\hline $\begin{array}{l}\text { UTSIDE TENPERATURE } \\
\text { OUSTIDE TOI A) }\end{array}$ & $3 / 18 / 97$ & 1132 & 56 & & $\pi$ \\
\hline
\end{tabular}

* Call weather station for required information at 373-2875. Note: If readings are outside designated ranges, Notify Test Director. See $\log$ Book Eutry $3 / 0 / 97$ - 1940 Has Ot of Spees on Date sheet, 10 . Sot Btot spees per OTP. iec 0730 Duta. 
HNF-SD-WM-OTR-231, Rev O

Page 146 of 165

\section{WORKING COPY}

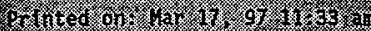

48-HOUR ON-LINE TEST DATA SHEET (Cont.)

OOR AIR DRYER/COOLING SKID

\begin{tabular}{|c|c|c|c|c|c|}
\hline RARAMEIER & GATE & Wiut & REPONG & EXPECTED RANGE & INI \\
\hline 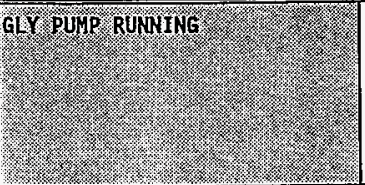 & $3 / 18 / 17$ & 1133 & , & 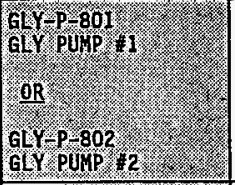 & $\pi c$ \\
\hline $\begin{array}{l}\text { GLY } 1-801 \text { (PI 701-A-17) } \\
\text { GY PP SUCT PRESS }\end{array}$ & $3 / 18 / 9$ & 1134 & 98 & $0-10 \mathrm{psI}$ & $\pi c$ \\
\hline $\begin{array}{l}\text { GLYPI } 803 \text { (PI- } 701 / 2=16) \\
G L Y \text { PP DISCH PRESS }\end{array}$ & $3 / 18 / 17$ & 1134 & 62 & 65.75 PSI: & $\pi x$ \\
\hline $\begin{array}{l}\text { GLY TI } 805 \text { (TI } 701 / 13) \\
\text { GLY CIRC PUAP SUCT TEP }\end{array}$ & $3 / 18 \%$ & 1134 & 113 & $100-120 \mathrm{~F}$ & th \\
\hline 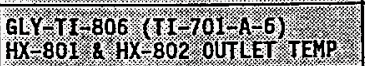 & $2 / 18 / 47$ & 1135 & 116 & $100-120 \%$ & tx \\
\hline 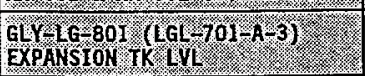 & $3 / 18 / 91$ & 1135 & & $1 / 3-2 / 3 \cdot 201$ & $\pi$ \\
\hline $\begin{array}{l}\text { 1A PT R 19, } \\
\text { IA THR } 801 \text { PRESS }\end{array}$ & $3 / 8 / 47$ & 1133 & & $80-105 . \mathrm{PSI}$. & tx \\
\hline $\begin{array}{l}\text { IA PIVR20 } \\
\text { IA PURGE PRESS }\end{array}$ & $3 / 18 / 67$ & 1,33 & 4 & $40-60.951$ & $\pi$ \\
\hline 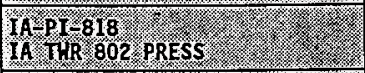 & $3 / 18 / 57$ & 133 & & $80=105.951$ & $\pm x_{1}$ \\
\hline 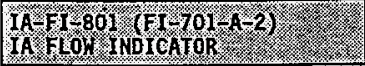 & $3 / 18 / 17$ & 1134 & 20 & $45=90 \mathrm{CrO}$ & $D_{C}$ \\
\hline 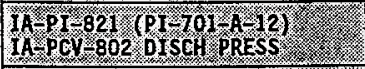 & $3 / 16 / 97$ & 11.34 & 42 & $30.45 \mathrm{pst}$ & $D<$ \\
\hline 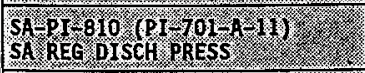 & $8 / 18 / 97$ & 1134 & 49 & $25=45$. 951 & $x$ \\
\hline $\begin{array}{l}\text { BLOW DOWN PREF IGIER, } \\
\text { ATIERTLLTER. REGENER TANISS }\end{array}$ & $3 / 19 / 97$ & & & & $D C$ \\
\hline lर & & & & 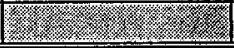 & \\
\hline
\end{tabular}

(1) Low Air usage 
HNF-SD-WM-OTR-231, Rev 0

Page 147 of 165

48-HOUR ON-LINE TEST DATA SHEET (Cont.)

FOR SA-CMP-802

\begin{tabular}{|c|c|c|c|c|c|}
\hline PARAMETER & DATE & THE & READING & EXPECTED $\mathrm{R}$ & INIT \\
\hline 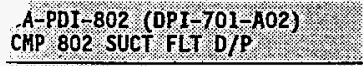 & $3 / 18 / 97$ & 1525 & -8 & 6. $\mathrm{psT}$ & \\
\hline $\begin{array}{l}\text { SA-PI } 802 \text { ( }(\mathrm{I}-701-\mathrm{A}=6) \\
\text { REMOTE BRG OIL PRESS }\end{array}$ & $3 / 18$ & 1525 & 22 & $15 \% 66$ & $\pi x$ \\
\hline $\begin{array}{l}\text { SA-PI } 806 \text { (PI-70I-A } 5 \text { ) } \\
\text { INTER STG DISCH PRESS }\end{array}$ & $3 / 1$ & 1526 & 40 & $20-40$ PSI & \\
\hline $\begin{array}{l}\text { GLY FG-806 (SFI 701 A- } 8 \text { ) } \\
S A-C H P-802 \text { HP SIDE GIY FLO }\end{array}$ & $3 / 18$ & 1526 & & FEOW & $C$ \\
\hline $\begin{array}{l}\text { SA-PI } 804 \text { (PI-701-A-8) } \\
\text { CMP } 802 \text { DISCH PRESS }\end{array}$ & $3 / 18 / 97$ & 1527 & 1073 & $85-105$ PST & $R$ \\
\hline $\begin{array}{l}\text { SA-TI-802 (TI-701-A-4) } \\
\text { CHP } 802 \text { SA DISCH TEMP). }\end{array}$ & $3 / 18$ & 1527 & & $90.120 \%$ & 72 \\
\hline $\begin{array}{l}S A-S G-802 \\
S A \text { CHP } 0 \mathrm{IL} L \mathrm{LV}\end{array}$ & & 1527 & & ABOVE IARK & $K$ \\
\hline $\begin{array}{l}\text { GLY-TI-804 (TI-701-A-10) } \\
\text { SA-CMP-802 GLY INLET TEMP. }\end{array}$ & & 1526 & 8 & $90-120 \mathrm{~F}$ & $\mathbb{K K}$ \\
\hline $\begin{array}{l}\text { GLY II-802 (II-701-A } 12 \text { ) } \\
\text { SA-CMP-802 GLY DISCH IEMP. }\end{array}$ & & 1528 & 111 & $90-120 \%$ & $D$ \\
\hline $\begin{array}{l}\text { Y FI-802 (FI-701-A } 1 \text { ) } \\
\text { A-CFP=802 SYS GLY FLO IND }\end{array}$ & & 1528 & 48 & $\angle 67 \cdot \mathrm{GPH}$ & $\pi$ \\
\hline $\begin{array}{l}\text { SA-PISL- } 801 \text { (PI-7OI-A-1) } \\
\text { SA HDR LO PRESS ALARH) }\end{array}$ & & 1529 & 100 & $90-100 \mathrm{ps}$ & $\not x$ \\
\hline $\begin{array}{l}\text { SA-TII-805 (TI 701 A-5) } \\
\text { SERVICE AIR HDR TERP). }\end{array}$ & & 1529 & 106 & $90-120 \%$ & D \\
\hline $\begin{array}{l}\text { DUTY CYCLE } \\
\text { (LOAD TIME/TOTAL TIME) }\end{array}$ & & 1525 & $25 / 95$ & . & $7 x$ \\
\hline RELATIVE HUMIDIYY $*$, & $3 / 1 8 \longdiv { 9 7 }$ & & (3). & 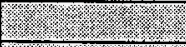 & \\
\hline ATMOSPHERIC PRESSURF . $*$ - & $\sqrt [ 3 ] { 1 8 } \longdiv { 9 7 }$ & & (3) & (2) & \\
\hline INSIDE TEMP. (IN $701-A)$ & $3 / 18 / 17$ & 1530 & 76 & 1. & Tz \\
\hline $\begin{array}{l}\text { OUTSIDE TEMPERATURE } \\
\text { (OUTSIDE TOI A) }\end{array}$ & $3 / 18 / 97$ & & & & $f x$ \\
\hline
\end{tabular}

* Call weather station for required information at $373-2875$

Note: If readings are outside designated ranges, Notify Test Director.

(1) See $\log$ Rook Eutiy - 3/10/97 - 1940 HA.

(2) Dut of Spees. on Date sleet, Not Dot of specs. Per OTP.

(3) See 0730 Dkik 
HNF-SD-WM-OTR-231, Rev 0

Page 148 of 165

\section{WORKING COPY}

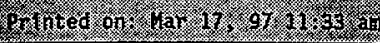

48-HOUR ON-LINE TEST DATA SHEET (cont.)

FOR AIR DRYER/COOLING SKID

\begin{tabular}{|c|c|c|c|c|c|}
\hline PARAMEIER & DATE & ME & REFDING & EXPECTEO RANGE: & INH \\
\hline GI PUMP RUNING & $3 / 18 / 97$ & $153 \mid$ & & 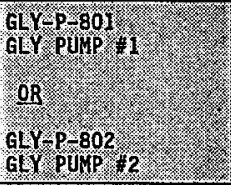 & PC \\
\hline 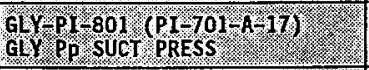 & $3 / 18 / 97$ & 1531 & 9.1 & $0-10$ PSI & $D c$ \\
\hline $\begin{array}{l}\text { GqY P I-803 (PI- } 101-\mathrm{A}-16) \\
\text { GLY PP DISCH PRES }\end{array}$ & $3 / 18 / 97$ & $\mid \sqrt{3} 1$ & 67 & $65-75 \mathrm{PSI}$ & $T$ \\
\hline 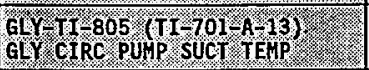 & $3 / 18 / 47$ & 1532 & 110 & $100-120 \%$ & toc \\
\hline 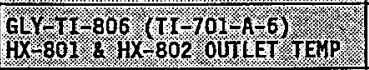 & $3 / 18 / 97$ & 1532 & & $100-120 \%$ & $7 c$ \\
\hline 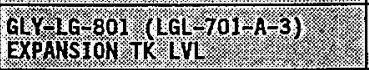 & $3 / 18 / 97$ & 1532 & & $1 / 3=2 / 3.7$ & $T \pi$ \\
\hline 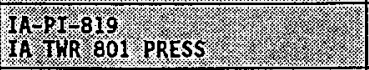 & $3 / 18 / 97$ & 1530 & & $80=105 \mathrm{PS}$ & Pe \\
\hline $\begin{array}{l}\text { 1APr } \\
\text { MA PURGE PRESS }\end{array}$ & $2 / 18 / 97$ & 1530 & & $40-60 \cdot 751$ & $x_{c}$ \\
\hline IA TKR 802 PRESS & $3 / 18 / 47$ & 1530 & & $80-105 . p 5$ & $P e$ \\
\hline 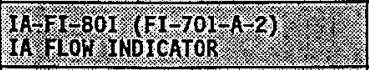 & $3 / 18 / 9$ & 1501 & 200 & 45-9 CFH & $T x$ \\
\hline 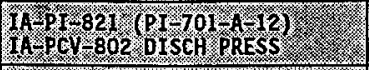 & $3 / 18 / 97$ & 1532 & 1 & $30-45.951$ & $7 x$ \\
\hline SARRE DISCH PRESS & $3 / 18 / 97$ & 1532 & 39 & $25+45 \cdot$ PSi & $\pi c$ \\
\hline 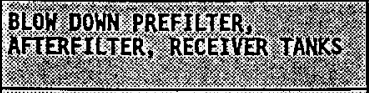 & $3 / 18 / 97$ & 1502 & & & TKC \\
\hline 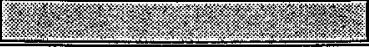 & & & & 2 & \\
\hline
\end{tabular}

(1) Low tit Usage. 
HNF-SD-WM-OTR-231, Rev 0

Page 149 of 165

\section{WORKINE CORY}

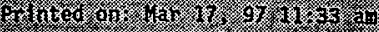

48-HOUR ON-LINE TEST DATA SHEET (Cont.)

FOR SA-CMP-802

\begin{tabular}{|c|c|c|c|c|c|}
\hline ARANETER. & DATE & $16 \mathrm{~L}$ & READIFG & EXPEGTE & 111 \\
\hline 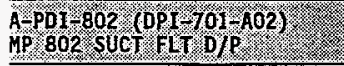 & $3 / 18 / 97$ & 1925 & 2.8 & K. pst & $R \times B$ \\
\hline $\begin{array}{l}\text { A-PI } 802 \text { (PI } 701 \text { A } 6 \text { ) } \\
\text { EMOTE BRG OIL PRESS }\end{array}$ & $3 / 18 \mid$ & 1925 & 23 & $15=6 \%$ & $\operatorname{Rr} B$ \\
\hline $\begin{array}{l}\text { A-PI-806 (P1 701-A } 5) \\
\text { NIER STG DISCH PRESS }\end{array}$ & $3 / 18 / 97$ & 1926 & 36 & $20-40$ & $R_{N} \beta$ \\
\hline $\begin{array}{l}\text { YYG } 806 \text { (SFI } 701-A-8) \\
\text { A-CMP } 802 \text { HP SIDE GLY FI0. }\end{array}$ & $3 / 18$ & 1927 & & Fon & RREB \\
\hline $\begin{array}{l}\text { A PI } 1804 \text { (PI } 101 \text { A } 8 \text { ) } \\
\text { MP } 802 \text { DISCH PRESS }\end{array}$ & $3 / 18 / 97$ & 1927 & U & $85-105$ & CNSB \\
\hline 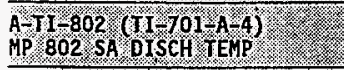 & $3 / 18 / 77$ & 1928 & $190(1)$ & $90 \times 120$ & RNS \\
\hline $\begin{array}{l}\text { A-SG } 802 \text {. } \\
\text { A CHP } 01 \text { LVL. }\end{array}$ & $3 / 18$ & 1928 & & ABOVE I & $R N B$ \\
\hline 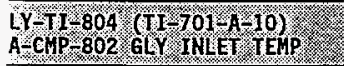 & $3 / 18 / 97$ & 1928 & $11^{\circ}$ & $90-120$ & $R_{A i B}$ \\
\hline $\begin{array}{l}\text { LY TI } 802 \text { (TI } 701-A-12) \\
A-C H P=802 \text { G }\end{array}$ & $3 / 18 / 97$ & 1928 & 114 & $90-120$ & $R \times B$ \\
\hline 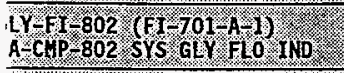 & $3 / 18 / 97$ & 1928 & 148 & K67. GPH & Rus \\
\hline 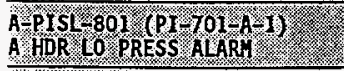 & $3 / 18 / 87$ & 1929 & & 90. $100 \mathrm{pg}$ & RNS \\
\hline 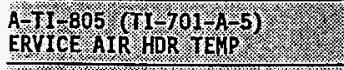 & $3 / 18$ & 1929 & & $90 \div 120$ & Res \\
\hline $\begin{array}{l}\text { UTY crgle } \\
\text { LOAD THE TOTA TNE }\end{array}$ & $3 / 18$ & 1.925 & 20 & & exs \\
\hline ELAIVE HUMIOIY & $=31,8197$ & 29 & $57 \%$ & & $84 B$ \\
\hline THOSPHERIC PRESSURE & $3 \operatorname{lig} 157$. & $7+3 z^{2}+10$ & $29.3,+2 * 200$ & & $2 \times 3$ \\
\hline 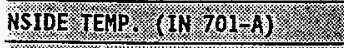 & $3 / 18 / 97$ & (925) & 73 & & RUS \\
\hline $\begin{array}{l}\text { UTSTDE TEMPERTIUR } \\
\text { OUTSIDE TOL }\end{array}$ & $3 / 18 / 97$ & 930 & 48 & & $R H^{S}$ \\
\hline
\end{tabular}

* Call weather station for required information at $373-2875$

Note: If readings are outside designated ranges, Notify Test Director.

(1) Soe Jeg Book entry-3/10/77-19 wo Hes

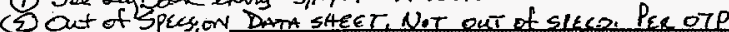

1430 Wen leteat reptifis

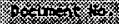

3. 17.26.0.6. 


\section{WORKING COPY}

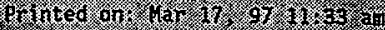

\section{8-HOUR ON-LINE TEST DATA SHEET (Cont.)}

FOR AIR DRYER/COOLING SKID

\begin{tabular}{|c|c|c|c|c|c|}
\hline PARAETER & BATE & STE & READING & EXPGSED RA & IIIJ \\
\hline GIV PMAP RUNING & $3 /, 8 / 47$ & 1930 & $\neq$ & 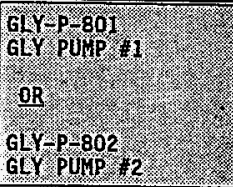 & $8 \times$ \\
\hline $\begin{array}{l}\text { GLY PI } 801 \text { (PI- } 701-A-17) \\
\text { GY PP SUCT PRES }\end{array}$ & $3 / 18 / 97$ & 1931 & 9.0 & $0.10 \mathrm{PSI}$ & \\
\hline 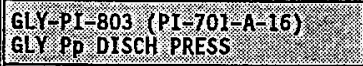 & $3 / 18197$ & 1931 & 62 & $65-75 \cdot \operatorname{PI}$ & \\
\hline 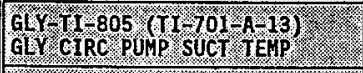 & $3 / 18197$ & 1931 & 114 & $100-120 \%$ & \\
\hline 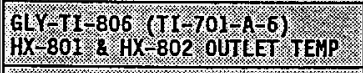 & $3 / 18 / 97$ & 1932 & 114 & $100-120 \%$ & \\
\hline 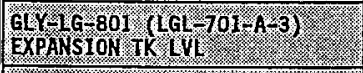 & $3 / 18 / 97$ & 1933 & & $1 / 3-2 \%$ & \\
\hline Ma PT 819 . 801 PRES & $3 / 18 / 97$ & 1933 & 100 & 80-105 PSI & \\
\hline 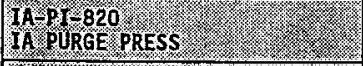 & $3 / 18 / 97$ & 1933 & 45 & $40=60 . \mathrm{PST}$ & \\
\hline 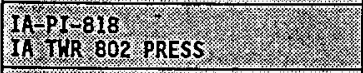 & $3 \ln \operatorname{lag}$ & 1933 & $\infty$ & $80 \%$ PS & \\
\hline $\begin{array}{l}\text { 1A } \\
\text { IA F }\end{array}$ & $3 / 18 / 57$ & 1934 & 20 & $45-90.964$ & \\
\hline 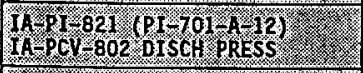 & $3 /, 2 k ?$ & 1939 & 43 & $30=45 \times$ PSI & \\
\hline 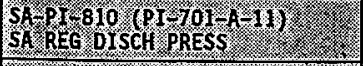 & $3 / 18 / 97$ & 1934 & 40 & $25 \times 45 \cdot 9 S$ & \\
\hline 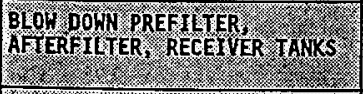 & $3 / 1847$ & 13 & $\sim$ & (3) & $\begin{array}{l}V \\
\& \beta\end{array}$ \\
\hline L & & & & (2.7. & \\
\hline
\end{tabular}

(1) Low Air lsate 
HNF-SD-WM-OTR-231, Rev 0

Page 151 of 165

\section{WORKING COPY}

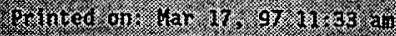

48-HOUR ON-LINE TEST DATA SHEET (Cont.)

FOR SA-CHP-802

\begin{tabular}{|c|c|c|c|c|c|}
\hline PRAYEIER & DATE & 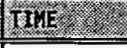 & REAGIY & EXPEOTED RANGE. & 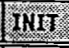 \\
\hline 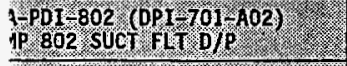 & $3 / 18 / 97$ & 2320 & $2^{1.8} 803$ & K6. PST. & $8 x+3$ \\
\hline 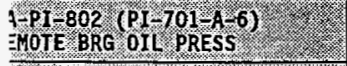 & & 2320 & 22 & 15.46 .65 & \\
\hline $\begin{array}{l}\text { PRI } 806 \text { (PI 701/A } 5) \\
\text { ITER STG DISCH PRESS }\end{array}$ & & 2321 & 29 & $20=40$ & \\
\hline 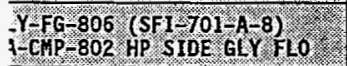 & & 2321 & $\checkmark$ & Row & \\
\hline 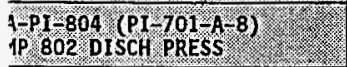 & & 2321 & 108 (1) & $85 \cdot 105$ & \\
\hline $\begin{array}{l}\text { 4.TI } 802 \text { (TI-701) } 4 \text { ) } \\
\text { YP } 802 \text { SA DISCH IEMP }\end{array}$ & & 2321 & 188 & $90-120$ & \\
\hline $\begin{array}{l}\text { SG } 802 \text {, } \\
\text { GMP } 0 \mathrm{IL} \text { LVL }\end{array}$ & & 2322 & $\checkmark$ & ABOVE MA & \\
\hline 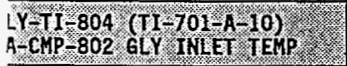 & & 2322 & 112 & 90.120 & \\
\hline 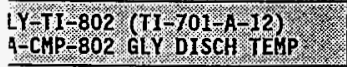 & & 2322 & $112^{\circ}$ & 90.120 & \\
\hline 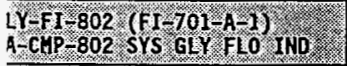 & & 2323 & 48 & 67. GP & \\
\hline 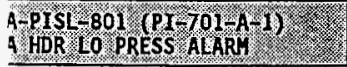 & & 2323 & 95 & $90 \% 100$ PST & \\
\hline 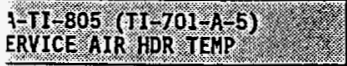 & & 2323 & 108 & $90-120$ & \\
\hline $\begin{array}{l}\text { WTY CYCLE } \\
\text { WOAD THE TOTA, - THE) }\end{array}$ & & 2320 & & & \\
\hline EATIY HUHDIV & & 2323 & $57 \% 1430 \%$ & (1) & \\
\hline THOSPHER IC PRES SURE, / & & 2323 & $29.32^{*} \cdots 3$ & & \\
\hline 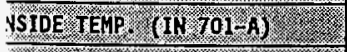 & & $235^{3}+163$ & 76 & & \\
\hline 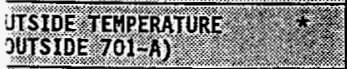 & $3 / 18197$ & 2324 & 52 & . & $\begin{array}{r}7 \\
R+3\end{array}$ \\
\hline
\end{tabular}

* Call weather station for required information at 373-2875

Note: If readings are outside designated ranges, Notify Test Director.

(i) out of spees. IN DATA SNEET, NDT onT \&T XECS PER OTP.

(2) SEE Loq Book Exir Y oN 3/10/97-1940 HRs

\begin{tabular}{|c|c|c|}
\hline $\begin{array}{ll} \\
7\end{array}$ & 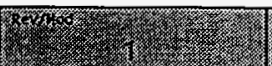 & 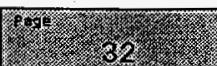 \\
\hline
\end{tabular}


48-HOUR ON-LINE TEST DATA SHEET ( Cont.)

FOR AIR DRYER/COOLING SKID

\begin{tabular}{|c|c|c|c|c|c|}
\hline PARAMLIER & DATE & TIE & READING & SXPECTED RAN & INIT \\
\hline 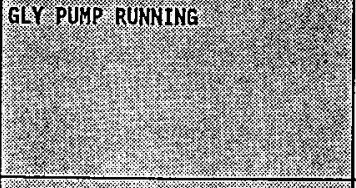 & $3 / 18 / 97$ & 2325 & $\neq 1$. & 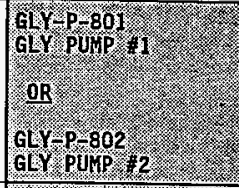 & Cos \\
\hline $\begin{array}{l}\text { GY P P }=801 \text { (PI } 701-A-17) \\
G L P P \text { SUCT PRESS }\end{array}$ & 1 & 2325 & 9.0 & 0-10.PSI & \\
\hline $\begin{array}{l}\text { GLY I } 803 \text { (PI } 701-16) \text { ) } \\
\text { GIY PD DISCH PRESS }\end{array}$ & & 2325 & 60 & $65-75 \mathrm{PST}$ & \\
\hline 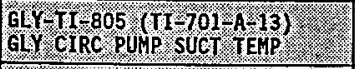 & & 2325 & 112 & $100-120 \%$ & \\
\hline 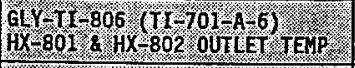 & & 2325 & $1 / 2$ & $100-120 \%$ & \\
\hline 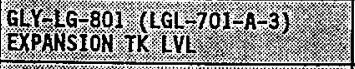 & & 2326 & & $1 / 3-2 / 3.90$ & \\
\hline WA P I 819 TR 801 PRESS & & 2326 & 98 & 80. 105. PST & \\
\hline TA P T 820 . PRESS & & 2326 & 98 & $40=60 . P S I$ & \\
\hline 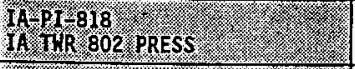 & & 2326 & 98 & $80-905$ PSI & \\
\hline 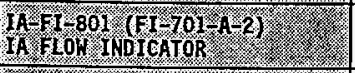 & & 2327 & 20 & $45-90 . \mathrm{CF}$ & \\
\hline 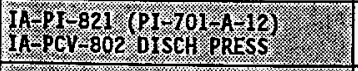 & & 23.27 & 42 & $30=45.951$ & \\
\hline 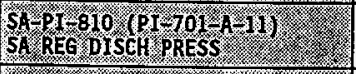 & & 2329 & 40 & $25-45, \mathrm{PSI}, 0$. & \\
\hline 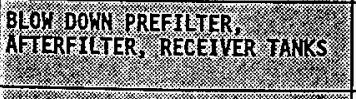 & $\begin{array}{c}1 \\
3 \log / 97\end{array}$ & $233 j$ & $\checkmark$ & 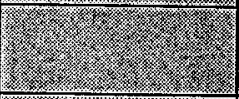 & $\frac{b}{a+3}$ \\
\hline 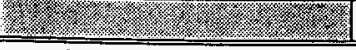 & & & & (2) & \\
\hline
\end{tabular}


HNF-SD-WM-OTR-231, Rev 0

Page 153 of 165

\section{WORKING COPY}

(6.

48-HOUR ON-LINE TEST DATA SHEET (Cont.)

FOR SA-CMP-802

\begin{tabular}{|c|c|c|c|c|c|}
\hline BRAMETER & DATE & 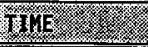 & READING & EXPECTED RANLE & SNII \\
\hline 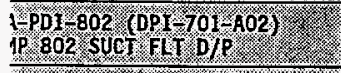 & $3 / 19 / 97$ & 0320 & 1.8 & Ko pst & RNB \\
\hline 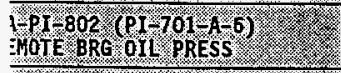 & & 0320 & 22 & $15 \% 40.6 \%$ & \\
\hline $\begin{array}{l}\text { MPI BO6 (PI-701/A S) } \\
\text { ITER STG DISCH PRESS }\end{array}$ & & 0320 & 28 & $20 \% 40 \%$ & \\
\hline 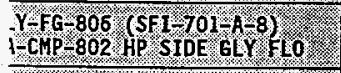 & & 0320 & $\checkmark$ & $\mathrm{FlOH}$ & \\
\hline $\begin{array}{l}\text { IPI-804 (PI-701-4-8) } \\
\text { 1P } 802 \text { DISCH PRESS }\end{array}$ & & 0320 & 108 & $85=105 \%$ & \\
\hline $\begin{array}{l}\text { PTI-802 (II } 701-4 \text { A) } \\
\text { P } 802 \text { SA DISCH TEP }\end{array}$ & & 0321 & 188 & $90-120 \%$ & \\
\hline $\begin{array}{l}\text { HSG } 802 \\
\text { TCM } 0 \mathrm{LL} \mathrm{LVL}\end{array}$ & & 0321 & $\checkmark$ & ABOVE Y & \\
\hline 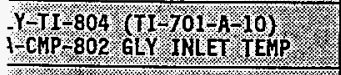 & & 0321 & 111 & 90.120 & \\
\hline 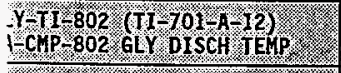 & & 0321 & 111 & $90=120$ & \\
\hline 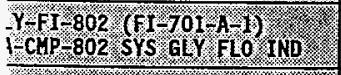 & & 0322 & SD & K67: GPF & \\
\hline $\begin{array}{l}\text { WPISE } 801 \text { (PI TOI A I) } \\
\text { HDR LO PRESS ALARH }\end{array}$ & - & 0322 & 98 & $90-100.95$ & \\
\hline 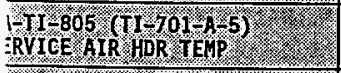 & & 322 & 106 & 90 1 $20 . \mathrm{F}$. & \\
\hline $\begin{array}{l}\text { MT CYCME } \\
\text { OAD TIME TOLA! TIKE) }\end{array}$ & & 0320 & $0=$ & & \\
\hline LATVE HUMIDUY & & 0322 & $57 \% \quad 1430 x$ & & \\
\hline HOSPHER IC PRESSURE & & 0323 & $29.32 \% 143000$ & & \\
\hline ISIDE TEP (IN $701 \%$ ) & & 0323 & 76 & & \\
\hline $\begin{array}{l}\text { ISIDE TEMPEATIURE } \\
\text { UTSIDE }(01 / \mathrm{A})\end{array}$ & $3 / 19 / 97$ & 0323 & 50 & (7) & R+B \\
\hline
\end{tabular}

* Call weather station for required information at $373-2875$

Vote: If readings are outside designated ranges, Notify. Test Director.

Out of specs. on Dart SHEET NoT OXT of spics. pEe OTP.

SEE Log $800 k$ ENTH on $3 / 10 / 97-1940$ HLS. 


\section{WORKING COPY}

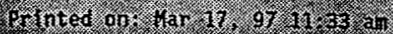

48-HOUR ON-LINE TEST DATA SHEET (Cont.)

FOR AIR DRYER/COOLING SKID

\begin{tabular}{|c|c|c|c|c|c|}
\hline PARANEIER & MIE & 105 & REFOING & EPEGT ED RRNGE & Wnir. \\
\hline GL PUP RUNING ( & $3 / 19 / 97$ & 0323 & & 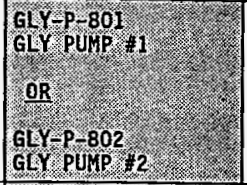 & lus \\
\hline $\begin{array}{l}\text { GLY P I } 801 \text { (PI-TO1 R - TT) } \\
\text { GLY PP SUCT PRESS }\end{array}$ & & 0323 & 9.0 & $0=10 \mathrm{ps}$ & \\
\hline 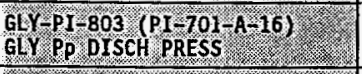 & & 0323 & 60 & 65-75. PSI & \\
\hline 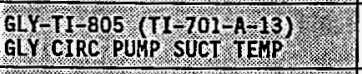 & & 0324 & 112 & $100-120.9$ & \\
\hline 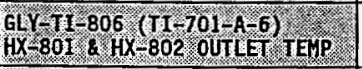 & & 0324 & 112 & $100-120 \%$ & \\
\hline 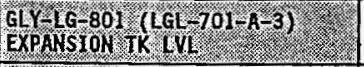 & & 0324 & & $1 / 3=2 / 3 \cdot \operatorname{Rv}$ & \\
\hline 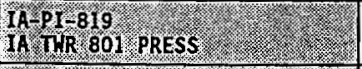 & & 0324 & 100 & $80 \cdot 105.951$ & \\
\hline $\begin{array}{l}\text { IA PT-820 } \\
\text { IA PURGE PRESS }\end{array}$ & & 0325 & 45 & $40-60 . \mathrm{pST}$ & \\
\hline 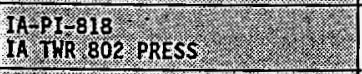 & & 0.325 & 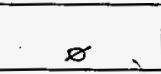 & $80-105 \times 5 \mathrm{~T}$ & \\
\hline $\begin{array}{l}\text { Mard } \\
\text { HA }\end{array}$ & & 25 & $\mathrm{zo}^{\mathrm{C}}$ & 45-90 cFH & \\
\hline 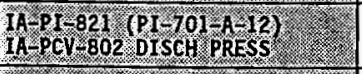 & & 0.325 & 42 & 30,45 PSI & \\
\hline 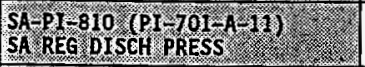 & & 325 & 40 & $25.45 \times \mathrm{pST}$ & \\
\hline 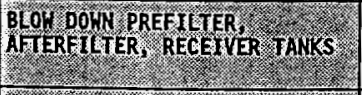 & & 0326 & & 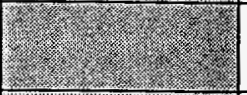 & $8+3$ \\
\hline (6) & $3 / 1967$ & & & (1) & \\
\hline
\end{tabular}


HNF-SD-WM-OTR-231, Rev 0

Page 155 of 165

\section{WORKING COPY}

f

48-HOUR ON-LINE TEST DATA SHEET (cont.)

FOR SA-CHP-802

\begin{tabular}{|c|c|c|c|c|c|}
\hline ARAMEIER & DATE & MIN & $\mathrm{REFHIT}$ & EXPEOLE RANGE & \\
\hline 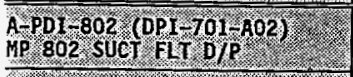 & $3 / 19 / 97$ & 0124 & 2.0 & R6 PSi & \\
\hline 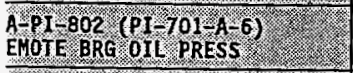 & $3 / 19 / 91$ & 6724 & 22 & $15-6$ & \\
\hline $\begin{array}{l}\text { API } 606 \text { (PI 701 A } 5) \\
\text { NTER STG DISCH PRESS }\end{array}$ & $3 / 19 / 97$ & 0.724 & 29 & & \\
\hline 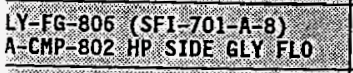 & $3 / 19$ & $07 u_{4}$ & & & \\
\hline 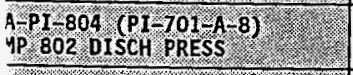 & & 074 & 1100 & 8 & \\
\hline $\begin{array}{l}\text { II } 802 \text { (I } 701 \text { A }_{4} \text { ) } \\
\text { P } 802 \text { SA OISCH TEY }\end{array}$ & $3 / 19 / 92$ & o?2L & (2) & & \\
\hline 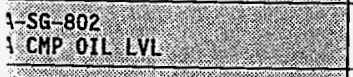 & $3 / 19 / 97$ & 6727 & & & \\
\hline 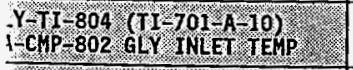 & $3 / 19 / 97$ & 0127 & 100 & 10 & \\
\hline 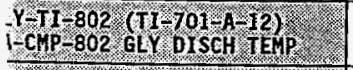 & $3 / 19 / 97$ & 0727. & 101 & & \\
\hline 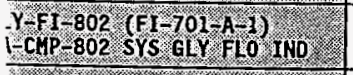 & $219 / 97$ & 0727 & 49 & $667.6 P H$ & \\
\hline 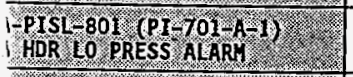 & $3 / 19 / 97$ & 0727 & 99 & $90 \% 100 \times s 1$ & , \\
\hline 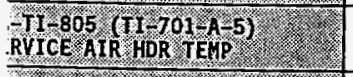 & $3 / 1 9 \longdiv { 9 7 }$ & 0727 & 98 & 90-120 & in \\
\hline $\begin{array}{l}\text { TY CVCEE } \\
\text { OAD THOLTAL TLE) }\end{array}$ & $3 / 19 / 2 ?$ & 0225 & $\begin{array}{l}27 \mathrm{sec} / \mathrm{GT} \\
\mathrm{LSec}\end{array}$ & & \\
\hline ATIV YUMIOHY $/ 2 \%$ & $3 / 19 / 27$ & & 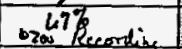 & & \\
\hline HOSPHERIC PRESSURE & $3 / 19 / 97$ & & 27000 Resaling & & \\
\hline 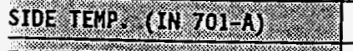 & $3 / 18 / 17$ & 1726 & $72^{\circ}$ & & \\
\hline $\begin{array}{l}\text { TSIDE TEMPEATTRE } \\
\text { UTSIDE } 701-2)\end{array}$ & $3 / 19 / 97$ & $\theta 726$ & $53^{\circ}$ & & \\
\hline
\end{tabular}

- Call weather station for required information at $373-2875$

ote: If readings are outside designated ranges, Notify Test Director.

lout of specs. on Data SHeat, NoT out of specsiper ot?.

:Sa-Legbeak Entry ow 3/10197-19401tRs.

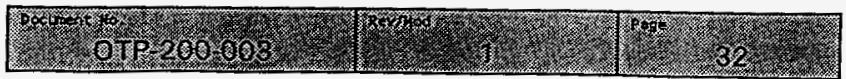


HNF-SD-WM-OTR-231, Rev 0

Page 156 of 165

\section{WORKINGCOPY}

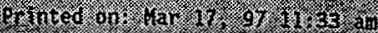

48-HOUR ON-LINE TEST DATA SHEET (cont.)

FOR AIR DRYER/COOLING SKID

\begin{tabular}{|c|c|c|c|c|c|}
\hline PARAMETER & BATE & 142 & REFING & EXPEGTED RANGE & 11 \\
\hline GIV RULP RUNGIG & $3 / 19 / 97$ & 0728 & 801 & 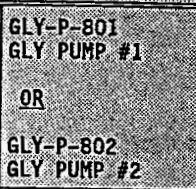 & \\
\hline 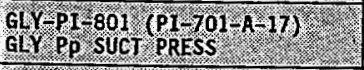 & $1 / 19 / 97$ & 0728 & 1 & 0.10 psi & \\
\hline $\begin{array}{l}\text { GLY PI-803 (PI-701-A-16) } \\
\text { GY RP DISCH PRESS }\end{array}$ & 31969 & 0728 & 60 & $65 \% 75$ rsi & \\
\hline 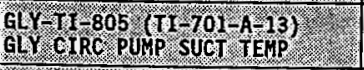 & $3 / 4 / 97$ & 6728 & 103 & $100=120$ 的 & \\
\hline $\begin{array}{l}\text { Gl } \\
H \mathrm{X}-801\end{array}$ & & 0728 & 103 & $100-120 \%$ & \\
\hline 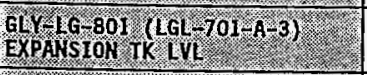 & $8 / 19 / 97$ & 0728 & 18 & $13-21 \%$ rol & \\
\hline 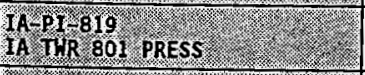 & & 0729 & 0 & $80.105 \times \mathrm{PST}$ & \\
\hline $\begin{array}{l}\text { WASPI } 820 \text {. } \\
\text { MA PUREE PRES }\end{array}$ & $3 / 197$ & 0729 & ts & $40=60 . \mathrm{PST}$ & $h$ \\
\hline 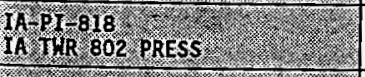 & $\sqrt[3]{19 / 77}$ & 0729 & 103 & $80-105 \cdot \mathrm{PS}$ & \\
\hline MA & $3 / 19 / 69$ & 0729 & 200 & $45.90 .97 \%$ & $D_{2}$ \\
\hline 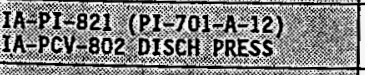 & $3 / 19 / 27$ & 0730 & 1 & 30.45 .951 & 10 \\
\hline 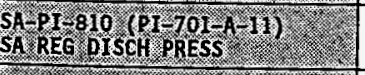 & $3 / 19 / 47$ & .0730 & 39 & $25 \% 55,351$ & \\
\hline BOW DOW RREITLTER. & $3 / 19 / 97$ & 0730 & & & \\
\hline 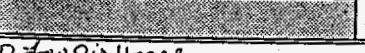 & & & & & \\
\hline
\end{tabular}

2.




\section{WORKINE GOPY}

\section{8-HOUR ON-LINE TEST DATA SHEET (Cont.)}

FOR SA-CMP-802

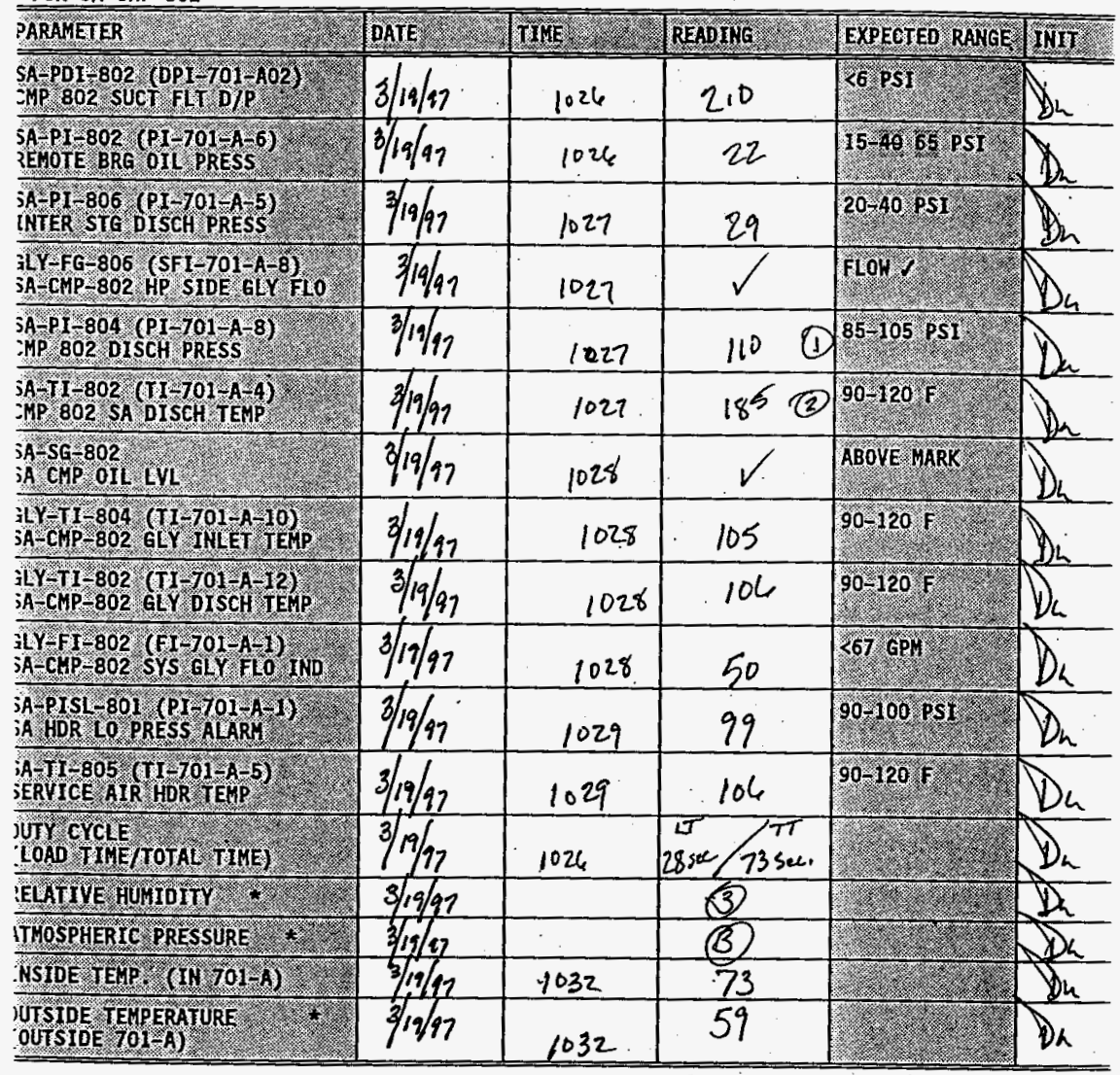

* Call weather station for required information at $373-2875$

Note: If readings are outside designated ranges, Notify Test Director.

ut of Spres. O DATr shect Not out of Spas. Fon OTP.

jee $\log$ Book Enty $3 / 1 / 497-1940$ HARS.

iex 0730 Pata. 
HNF-SD-WM-OTR-231, Rev 0

Page 158 of 165

\section{WORKME CORY}

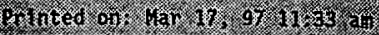

48-HOUR ON-LINE TEST DATA SHEET (Cont.)

OOR AIR DRYER/COOLING SKID

\begin{tabular}{|c|c|c|c|c|c|}
\hline PARANETER & OAI & 110 & SEATHS & EXPCTER RANGE & \\
\hline GL PUMP RUNALG ( & $3 / 19 / 57$ & 1030 & 801 & 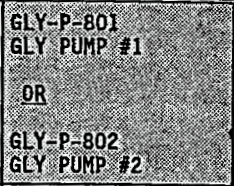 & \\
\hline 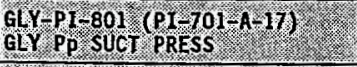 & $3 / 1969$ & 1030 & 3 & 0.10.psI & \\
\hline GLY Y T R 803 (PI-7O1-A-16) & & 1030 & 60 & $65-75$ PST & \\
\hline 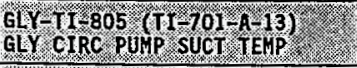 & & 1030 & & $100-120$ & \\
\hline 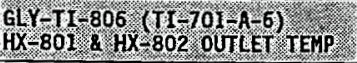 & & 1030 & 107 & $100 \times 120 \mathrm{r}$ & \\
\hline 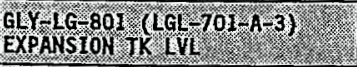 & & 1030 & & $1 / 3=2 / 7$ iul & \\
\hline $\begin{array}{l}\text { IA PI } 819 \\
\mathrm{AA} T \mathrm{HR} 801 \text { PRESS }\end{array}$ & $3 / 199$ & 1030 & & $80-105$ PsI & \\
\hline 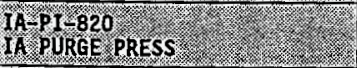 & & 1030 & & $40 \cdot 60.95$ & \\
\hline IF THR 802 PRESS & $3 / 19 / 97$ & 1050 & 103 & 6o -105 PsI & \\
\hline 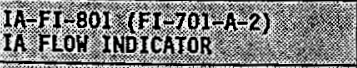 & & 1031 & 20 & 45-90 chit & \\
\hline 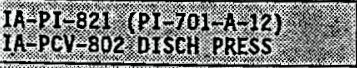 & 31910 & 1031 & 41 & $30=45, \mathrm{PSI}$ & \\
\hline 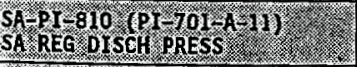 & $3 / 19 / 11$ & 1031 & 29 & 25.45 .159 & \\
\hline BLO DOWH RRE UMTER, & $3 / 1969$ & 10.31 & & 1. & \\
\hline 10 & & & & 10. & \\
\hline
\end{tabular}

1.7.




\section{ATTACHMENT 2 OTP EXCEPTION LOG}

OTP EXCEPTION SHEET NUMBER 1 (Make copies of this page as necessary)

Procedure Step: 5.1 .26

Description of Problem:

UNLOAD/LOAD SIBNAL FEED IS FROM AIRHEAOER,

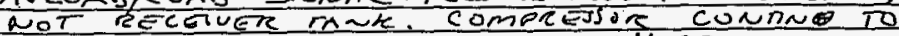

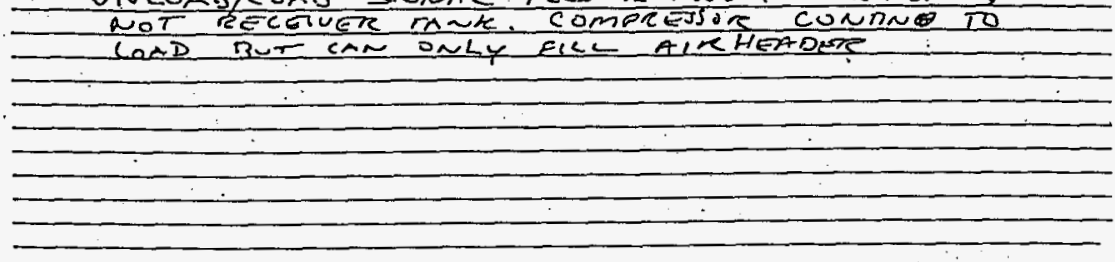

Exception Resolution:

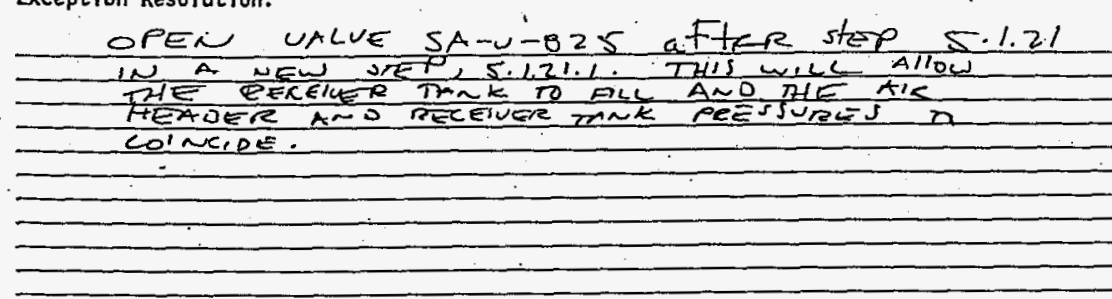

Date Exception Resolved: $3 / 3 / 32$

Test Director Paceleullo

$3-3-47$

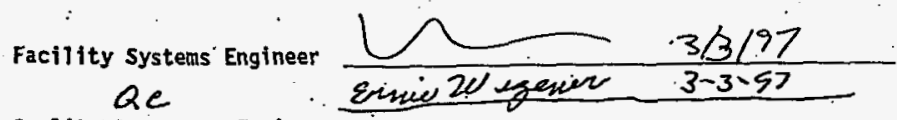

Qual ity Assurance Engineer

(NA for clarification) 


\section{ATTACHMENT 2}

\section{OTP EXCEPTION LOG}

OTP EXCEPTION SHEET NUMBER Z (Make copies of this page as necessary)

Procedure Step: $5.1 .38 / 5.1 .39$

Description of Problem:

$$
5.2 .4 / 5.2 .3
$$

VALUES in tarle 5.139 reguired to bo opened prior TO THE VALUES IN TXRLE S.1.38 THE VALUES POSITONED IN TABLE 5.139 /SOLATE ALR FROA TLE INTER

- SUSTEN. DUE TO THE AMUNNT OF NEE RERURED

To POSTRON TVE UALVS UN TABLE S.1.3S THE RELUER

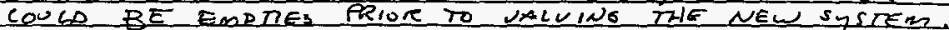

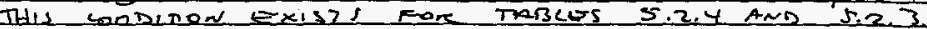

Exception Resolution:

PERFORM VALUING in SREP 5.139 BEFORE 51.38. AND

STER 5.2 .4 BEAORE 52.3 .

Date Exception Resoived: $3 / 4 / 97$

Test Director that Whull 34?

Facility Systems Engineer

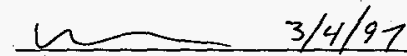

Qual ity Assurance Engineer

(NA for clarification)

Ye 


\section{MIORMATLN COPY}

\section{ATTACHMENT 2}

\section{OTP EXCEPTION LOG}

OTP EXCEPTION SHEET NUMBER 3 (Make copies of this page as necessary) Procedure Step: 5.2 .3$.

Description of Problem:

CLOSIMS VACUE SA-v-O25 ReU 5495.2 .3

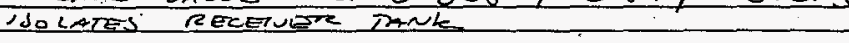

ixception Resolution:

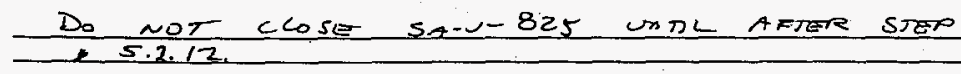

Date Exception Resolved: 3-10-97

Test Director Helereles

Facility Systems Engineer $Q \subset$

E.h. $3 / 10 / 92$

Quality Assurance Engineer

(NA for clarification) 


\section{ATTACHMENT 2 \\ OTP EXCEPTION LOG}

OTP EXCEPTION SHEET NUMBER 4 (Make copfes of this page as necessary)

Procedure Step: 5.2 .8

Description of Problem:

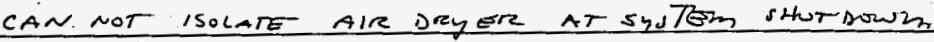

Exception Resolution:

Move step 5.2 .8 to follow 5.2.5. Thll wich

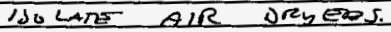

Date Exception Resolved: $3-10-97$

Test Director toufexude

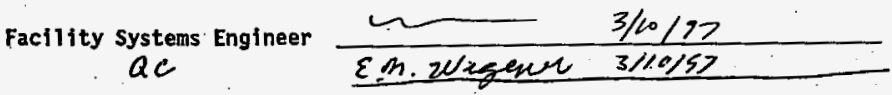

Quality Assurance Engineer

(NA for clarification) 


\section{ATTACHMENT 2}

\section{OTP EXCEPTION LOG}

OTP EXCEPTION SHEET NUMBER 5 (Make copies of this page as necessary)

Procedure Step: $5.1 .28,5.1 .54$

Description of Problem:

IA-PISL-801 should be SA-PISe-807

ixception Resolution:

CHANGE ITA-PISL-801 TO READ SA-pISL-bol in 5 TEPS $5.1 .2 y$ AND 5154 .

Date Exception Resolved: $3-1097$

Test Director Paulgaiello

Facility systems Engineer $3 / 30 / 97$

Quality Assurance Engineer

re (NA for clarification) 


\section{INFORMATION COPY}

\section{ATTACHMENT 2}

\section{OTP EXCEPTION LOG}

OTP EXCEPTION SHEET NUMBER 6 (Make copies of this page as necessary)

Procedure Step: AIR DRYER/COOLING SKID DATA SHEET

Description of Problem:

Instument air flow indicator, IA FI-801, belas exrected range. Expected range is 45 - $90 \mathrm{cfm}$.

Exception Resolution:

Expected air usage was predicted based on Round

Shut redings reconded dering summer of is56.

sace this fime, numenows. air wequivmmit have

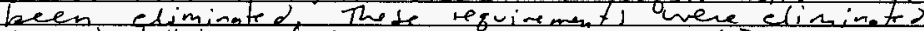

by installation of EथRAFS ANA SMALC CEATA which

weve repaired by $4-320,6-151$ AND W-OBO ALL

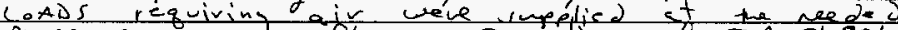

pressure and jor flow. oparation af IA-fleol

usas verified by temponarig open a dounstran

value to introdule a tampory load this load

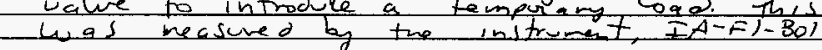

Date Exception Resolved: $6 / 24 / 27$

Test Director Paul Haule 6-24-97

Facility Systems Engineer $6 / 24 / 57$

Quality Assurance Engineer
(NA for clarification) 
HNF-SD-WM-OTR-231, Rev 0

Page 164 of 165

$164 \mathrm{~A}$

\section{INFORMATION COPY}

\section{ATTACHMENT 2 \\ OTP EXCEPTION LOG}

OTP EXCEPTION SHEET NUMBER 7 (Make copies of this page as necessary)

Procedure Step: VARIOUS

Description of Problem:

NUmERous signature blanks through out the operationatest prosedure were not completed during testing

Exception Resolution:

The signatures which were not recorded during testing can be found in the body of $t$. o th. The signatures weje not included as an ovensight, During revey of the test procedure a te testings it was jetermined that the signatures which weve not obtained during formal testing of the $701=A$ comp sor system have a neglicele impoct on the performance and gaveity of the test ond de not a ffect tor validit it twe pialts.

Date Exception Resolved: $6 / 24 / 92$

Test Director Paul Naulle 6-24.97

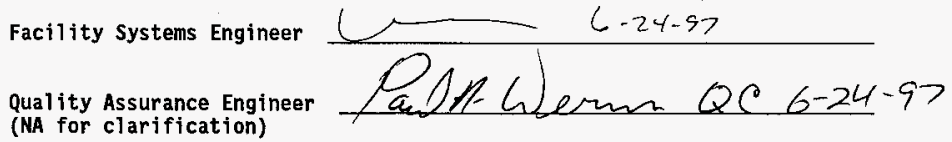




\section{ATTACHMENT 1}

\section{OTP ACCEPTANCE}

\section{Acceptance of Test Results}

The test has been completed and the results have been reviewed. Any exceptions discovered during the OTP have been satisfactorily recorded and resolved. The results of the Operability test of the 241-A-701 compressed air system and heat pump are accepted by the undersigned.

Support Systems Cognizant Engineer

Remey $L$ Powers

24I-A, 24I-AX Tank Farm Cognizant Engineer

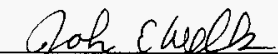

Eleftrical Cognizant Engineer

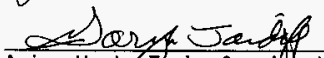

Aging Waste Janks Cogniffent Engineer

\section{We Adamol}

Quality Assurance Engineer

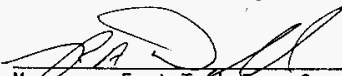

Madager, East Tańk Farm Operations

$$
\frac{6 / 24 / 97}{\text { Date }}
$$
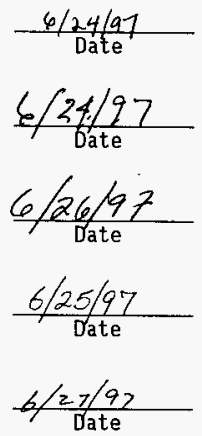